\title{
MicroBooNE: The Search For The MiniBooNE Low Energy Excess
}

\author{
David Kaleko
}

Submitted in partial fulfillment of the

requirements for the degree

of Doctor of Philosophy

in the Graduate School of Arts and Sciences

COLUMBIA UNIVERSITY

2017 
David Kaleko

All Rights Reserved 


\section{Table of Contents}

List of Figures $\quad$ iv

List of Tables $\quad$ xiv

\begin{tabular}{lll}
\hline & Introduction & 1
\end{tabular}

2 Neutrinos, Neutrino Oscillations, and Sterile Neutrinos 3

2.1 Introduction to Neutrinos $\ldots \ldots \ldots \ldots \ldots$

2.2 Neutrino Oscillations . . . . . . . . . . . . . . . . . . 4

2.3 Sterile Neutrinos $\ldots \ldots \ldots \ldots \ldots$

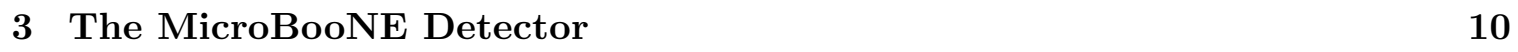

3.1 Introduction $\ldots \ldots \ldots \ldots \ldots \ldots$

3.2 Time Projection Chamber . . . . . . . . . . . . . . . . . . 12

3.3 Light Collection System . . . . . . . . . . . . . . . . . . . . 12

3.4 Electronics, Readout, and Triggering . . . . . . . . . . . . . . . . . . 14

4 The Booster Neutrino Beam 19

4.1 The Booster Neutrino Beam . . . . . . . . . . . . . . . . . . . . . . . . . . . 19

$4.1 .1 \quad$ Primary Proton Beam $\ldots \ldots \ldots$. . . . . . . . . . . . . . 19

$4.1 .2 \quad$ Proton Target and Focusing Horn $\ldots \ldots \ldots$. . . . . . . . 21

4.2 Monte Carlo Neutrino Flux Prediction . . . . . . . . . . . . . . . . . . . . . 23

\begin{tabular}{|lll}
5 & Low Energy Excess: LSND and MiniBooNE & 27
\end{tabular}

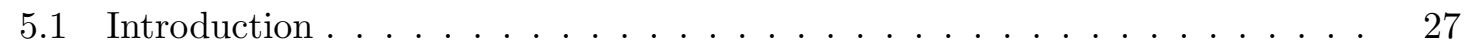




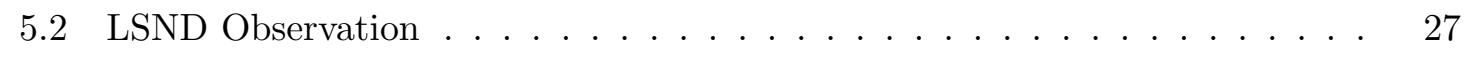

5.3 The MiniBooNE Experiment $\ldots \ldots \ldots \ldots \ldots \ldots$

5.3 .1 The MiniBooNE Detector and Monte Carlo Simulation . . . . . . 28

$5.3 .2 \quad$ MiniBooNE Event Selection . . . . . . . . . . . . . . . . . . . . . . 29

5.3 .3 MiniBooNE Results . . . . . . . . . . . . . . . . . . 32

5.3 .4 Proposed Low Energy Excess Sources $\ldots \ldots \ldots$

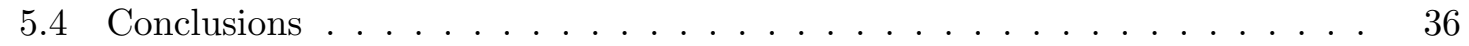

6 Low Energy Excess: MicroBooNE 38

$6.1 \quad$ MicroBooNE In The Context of the Low Energy Excess $\ldots \ldots \ldots$. . . . . 38

$6.1 .1 \quad$ Past Sensitivity Studies $\ldots \ldots \ldots \ldots$

6.2 Monte Carlo Simulation . . . . . . . . . . . . . . . . . . . . . . 43

$6.2 .1 \quad$ Simulated Background Samples . . . . . . . . . . . . . . . . . . 43

6.2 .2 Reconstruction . . . . . . . . . . . . . . . . . . . 43

6.3 Event Selection . . . . . . . . . . . . . . . . . . . . . 45

$6.3 .1 \quad$ Electron/Photon Separation Algorithm _. . . . . . . . . . . 46

6.3 .2 Signal Selection Algorithm $\ldots \ldots \ldots$

6.3 .3 Energy Reconstruction $\ldots \ldots \ldots \ldots \ldots$

6.4 Backgrounds $\ldots \ldots \ldots \ldots \ldots \ldots \ldots \ldots \ldots \ldots \ldots \ldots \ldots$

6.4 .1 Background Topologies . . . . . . . . . . . . . . . . 57

6.4 .2 Background Normalization . . . . . . . . . . . . . . . . 63

6.4 .3 Analysis Cuts and Results . . . . . . . . . . . . . . . . . 64

6.5 MiniBooNE Low Energy Excess Signal Modeling In MicroBooNE . . . . . . 66

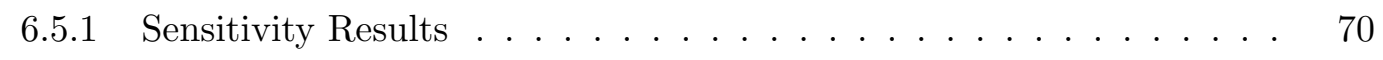

7 Studies of Kaons Produced at the Proton Target 76

7.1 BNB Kaon Production Introduction and Motivation . . . . . . . . . . 76

7.2 Event Selection . . . . . . . . . . . . . . . . . . . . . . . 80

7.2 .1 Track Reconstruction $\ldots \ldots \ldots \ldots \ldots$. . . . . . . . 82

7.2 .2 Selection Criteria . . . . . . . . . . . . . . . . . . 84

$7.2 .3 \quad$ Backgrounds $\ldots \ldots \ldots \ldots \ldots \ldots \ldots \ldots$ 
7.3 Neutrino Energy Reconstruction _. . . . . . . . . . . . . . . . 87

7.4 Results in Simulation $\ldots \ldots \ldots$. . . . . . . . . . . . . . . 90

7.5 Sideband Data and Simulation Comparisons . . . . . . . . . . . . . . . 93

7.6 Conclusions . . . . . . . . . . . . . . . . . . . . . 106

$\begin{array}{lll}8 & \text { Conclusions } & 108\end{array}$

\begin{tabular}{ll}
\hline Bibliography & 109
\end{tabular}

\begin{tabular}{lll}
\hline I Appendices & 114
\end{tabular}

\begin{tabular}{|l|l|}
\hline A Multiple Coulomb Scattering Publication & 115
\end{tabular} 


\section{List of Figures}

2.1 The neutrino mass hierarchy possibilities. The flavor eigenstate fraction for each mass eigenstate is shown by the relative amount of gray, red, or blue in

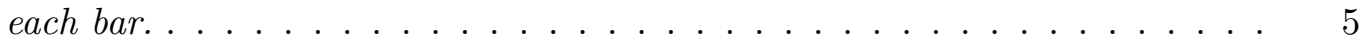

$2.2 \quad$ Results from the KamLAND collaboration measuring the survival probability of $\bar{\nu}_{e}$ from nuclear reactors. A clear oscillation as a function of $L / E$ as predicted by the two-neutrino model is shown. . . . . . . . . . . . . . . . . 7

2.3 Mass hierarchy with one heavy sterile neutrino included. The $\delta m^{2}$ from LSND is incorporated. . . . . . . . . . . . . . . . . . . . . . . . 9

3.1 A cartoon schematic of how a LArTPC works. Ionization electrons from particles traversing the detector medium are drifted by an electric field, $E_{\text {drift }}$ past multiple planes of sense wires. The signals on those wires create several two-dimensional images of the event, which are combined to create a threedimensional reconstruction of the event. Note that in a LArTPC, PMTs are used to collect scintillation light, but are not drawn in this diagram. . . . . . 11

3.2 A $3 D$ rendering of the MicroBooNE detector. The rectangular time projection chamber (TPC) fits within the cylindrical cryostat. The feedthroughs along the top allow for the PMT and sense wire signals to be read out to the DAQ. Not shown are the photomultiplier tubes (PMTS) located on the wall behind the sense wire planes. . . . . . . . . . . . . . . . . . . . . . . 13

3.3 A side-on view of the MicroBooNE detector showing the location of the 32 PMTs (labeled "optical units") and the four light guide paddles. . . . . . . . 15 
3.4 A picture of one of the 8 inch Hamamatsu R5912-02mod cryogenic photomultiplier tubes (PMT) used in the MicroBooNE detector. Note the clear acrylic plate, which is coated with a wavelength-shifting organic fluor (TPB) before installation to convert the VUV liquid argon scintillation light into the visible spectrum, detectable by the PMT. . . . . . . . . . . . . . . . . . . . . 16

3.5 The MicroBooNE readout schematic. On the left are portions of the readout operating at argon temperatures. Signals pass through feedthroughs into warm electronics readout boards, unique for the TPC (sense wire signals) and the light collection system (PMT signals). These signals are combined with external timing signals from the accelerator to form triggers that initiate readout of all systems. . . . . . . . . . . . . . . . . . . . . . 17

4.1 Overall layout of the BNB. The primary proton beam, extracted from the Booster, enters the target hall from the left. Upon exiting the target hall, particles encounter a 50-meter-long decay region, terminating in the beam stop on the right. . . . . . . . . . . . . . . . . . 20

4.2 The BNB focusing horn system. The gray outer conductor is drawn transparent for visualization purposes. The beryllium target lies within the central hollow tube axis. A current flows along the inner conductor, returning along the outer conductor. . . . . . . . . . . . . . . . . . 22

4.3 A cartoon diagram of the incident $8.89 \mathrm{GeV} / \mathrm{c}$ proton beam (from the left) colliding with the beryllium target within the focusing horn. Shown is the current configuration for the horn referred to as "neutrino mode" in which positive charged secondaries are focused into the decay region. The $25 \mathrm{~m}$ absorber drawn is removable, and was not used for the analyses described in this thesis. . . . . . . . . . . . . . . . . . . . 22

$4.4 \quad$ Neutrino flux prediction (black dots) with systematic error bars (red envelope) excluding proton delivery systematics which results in a flat normalization correction. . . . . . . . . . . . . . . . . . 26 
5.1 The MiniBooNE detector enclosure (left) and a cut-away drawing (right) of the detector showing the distribution of PMT's in the signal and veto regions. 29

$5.2 \quad$ A schematic of the pattern Cherenkov light from different particles would make projected onto the inner walls of the MiniBooNE detector. Top is a muon track (a filled-in ring), middle is an electron (a fuzzy ring), bottom is a photon that pair-produces and creates two fuzzy rings. . . . . . . . . . . . 30

$5.3 \quad$ Feynman diagrams of the charged-current quasi-elastic (CCQE) interaction channel for $\nu_{e}, \nu_{\mu}, \bar{\nu}_{\mu}$, and $\bar{\nu}_{e}$ (clockwise from the top left). $\nu_{e} C C Q E$ is the signal channel for the MiniBooNE oscillation analysis. . . . . . . . . . . . . 31

5.4 The $E_{\nu}^{Q E}$ distribution for MiniBooNE data (points with statistical errors) and and backgrounds (histogram with systematic errors). . . . . . . . . . . . 33

5.5 The MiniBooNE event excess as a function of $E_{\nu}^{Q E}$. Also shown are the expectations from the best oscillation fit and from neutrino oscillation parameters in the LSND allowed region. The error bars include both statistical and systematic errors. . . . . . . . . . . . . . . . . . . . . . 34

$6.1 \quad$ Energy loss per unit length along the first $2.4 \mathrm{~cm}$ of simulated single electron showers (red) and single photon showers (black) in terms of minimally ionizing particle (MIP) energy in liquid argon (about 2.1 MeV/cm). Photons in general have twice the $d E / d x$ of electrons at the start of their showers, stemming from pair production. . . . . . . . . . . . . . . . . . . . . . 41

6.2 The results of the first analysis to scale the MiniBooNE backgrounds and excess to MicroBooNE both under the assumption that the excess is due to an electron-like event (left) or under a photon-like event (right). Stacked histograms show the expected background. Error bars indicate statistical uncertainty. The number of signal events, scaled from MiniBooNE for neutrino flux and fiducial volume, is the same in both plots (though dedicate electronspecific and photon-specific event selection cuts may show this to be unrealistic). Both plots assume $6.6 \times 10^{20}$ POT for the MicroBooNE 60 ton fiducial

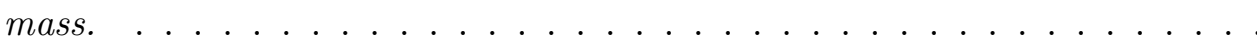


6.3 AlgoEMPart training results on perfect reconstructed electron showers and \begin{tabular}{|c|c|c|}
\hline on perfect reconstructed photon showers as described in Section & 6.3.1.1: $1 D$ \\
\hline
\end{tabular} landau + Gaussian fit to $d E / d x$. "Frac" in the legend is the relative normalization between the landau and Gaussian fits; that "Frac" is close to one means the landau fit dominates. . . . . . . . . . . . . . . . . . . . 48

6.4 AlgoEMPart: Computed 1D likelihood vs $d E / d x:$ red is photon, blue is electron. How the likelihood is computed is described in Section $\mid 6.3 .1 .1 .1 . . . . .550$

6.5 AlgoEMPart training results on perfect reconstructed electron and photon showers as described in Section |6.3.1.1 integrated over all energies: 2D likelihood distribution (radiation length vs. $d E / d x$ ). Low values of likelihood (purple) correspond to photon-like, high values (red) correspond to electronlike. . . . . . . . . . . . . . . . . . . 50

6.6 AlgoEMPart training results on perfect reconstructed electron showers as described in Section |6.3.1.1: Radiation length fit to single electron showers. Note the poor quality of the fit as the electron conversion distance for "perfect reconstruction" does not follow an exponential distribution; all conversion distances are below 0.3 centimeters. . . . . . . . . . . . . . . . . . . . . 51

6.7 AlgoEMPart training results on perfect reconstructed photon showers as described in Section |6.3.1.1: Radiation length fit to single photon showers. . . 52

6.8 AlgoEMPart: Computed 1D likelihood vs conversion distance (integrated over all energies): red is photon, blue is electron. How the likelihood is computed is described in Section|6.3.1.1] . . . . . . . . . . . . . . . . . . . . . . . . . 52

6.9 A flowchart depicting decisions the algorithm makes for each primary, noncosmic shower. If the algorithm gets to the bottom of the flowchart, that shower was determined to be from a $\nu_{e}^{C C}$ interaction, and a $\nu_{e}$ particle is created. For clarification of what some acronyms mean, see Figure 6.10 . . . 53

6.10 Schematic cartoons indicating how the signal selection algorithm makes decisions determining if two reconstructed showers are correlated, and if a reconstructed shower is correlated with a reconstructed track (as described in Figure 6.9p. . . . . . . . . . . . . . . . . . . . 54 
\begin{tabular}{|l|l|l|l|}
\hline 6.11 & Reconstructed neutrino energy as described in Section & 6.3 .3 & versus true neu-
\end{tabular}

trino energy. This plot was made from "perfect reconstruction" objects in
correctly identified $\nu_{e}^{C C Q E}$ events after all final analysis cuts were placed. . . 58

6.12 A neutrino energy resolution and bias plot. This is created by binning Figure

\begin{tabular}{|l|l|l|l|l|}
\hline & 6.11 & in true neutrino energy and making a distribution of (Reco Energy - \\
\hline
\end{tabular}

True Energy)/(True Energy). For each bin, the mean (red) and standard

deviation (blue) are plotted in the above figure. This plot was made from

"perfect reconstruction" objects in correctly identified $\nu_{e}^{C C Q E}$ events after all final analysis cuts were placed. . . . . . . . . . . . . . . . 59

6.13 A 3D event display showing measured cosmic tracks entering the MicroBooNE detector. The three boxes show the full readout window of the detector which corresponds to $4.8 \mathrm{~ms}$. The red highlighted box shows the physical volume of the TPC. The colored lines are 3D reconstructed cosmic tracks. Data taken in August, 2015. . . . . . . . . . . . . . . . . . . . . 62

6.14 The measured distribution of flash times (requiring flashes greater than 50PE) with respect to the trigger time for BNB-triggered events, shown as a ratio to the expected cosmic rate from off-beam data. The blue band denoting the cosmic rate was centered at one, with a width corresponding to the measured uncertainty in the cosmic rate. A clear excess can be seen due to neutrinos between 3 and $5 \mu$ s after the trigger. This is where the neutrinos were expected based on the RWM signal arrival time. A total of 1.92E6 BNB triggered events (unbiased trigger) were used to produce this plot. . . . . . . . . . . . 64

6.15 The backgrounds to the $\nu_{e}^{C C Q E}$ appearance search in MicroBooNE with statisticalonly error bars shown. The event selection is described in Section |6.3, the background topologies described in Section $\mid 6.4 .1$, the relative normalization between samples described in Section 16.4 .2 , and the energy reconstruction described in Section|6.3.3. . . . . . . . . . . . . . . . . . . . . . . 67

6.16 The computed distribution of $u_{z}$ (how forward-going the event is) vs. $E_{\text {vis }}$ for $N=1000$ times the MiniBooNE low energy excess (raw) events. . . . . 69 
6.17 The backgrounds to the $\nu_{e}^{C C Q E}$ appearance search in MicroBooNE with scaled \begin{tabular}{l}
\hline signal drawn. The event selection is described in Section |6.3. the background \\
\hline topologies described in Section $\mid$ 6.4.1, the relative normalization between sam- \\
\hline ples described in Section 6.4.2 and the energy reconstruction described in
\end{tabular} \begin{tabular}{|l|l|l|}
\hline ples described in Section & 6.4.2. and the energy reconstruction described in \\
\hline
\end{tabular}

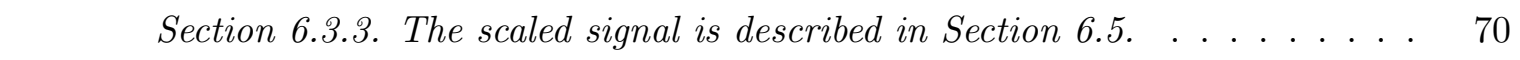

6.18 The backgrounds to the $\nu_{e}^{C C Q E}$ appearance search in MicroBooNE with scaled signal drawn. The event selection is described in Section 16.3 , the background topologies described in Section |6.4.1, the relative normalization between samples described in Section 6.4 .2 , and the energy reconstruction described in Section 6.3 .3 . The scaled signal is described in Section $\mid 6.5$. Here the shower reconstrution efficiency has been decreased from the "perfect reconstruction" value of $100 \%$ to $85 \%$ to emulate possible realistic shower reconstruction efficiencies. . . . . . . . . . . . . . . . 74

7.1 The breakdown of $\nu_{e}^{C C}$ interactions in MicroBooNE from the booster neutrino beam-line by $\nu_{e}$ parentage. $\nu_{e}$ from $K^{+}$decay is the primary contributor to

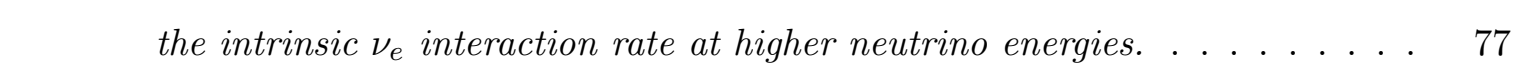

7.2 The Booster Neutrino Beam (BNB) flux at MicroBooNE. . . . . . . . . . . 78

7.3 The beam $\nu_{\mu}$ parentage as a function of true neutrino energy. . . . . . . . . 79

7.4 The fractional error for the BNB $\nu_{\mu}$ flux through MicroBooNE, broken up by systematic source. "Other" includes hadronic interactions, horn current uncertainty, skin depth effects, etc. The production uncertainties are divided by neutrino parent. Note the largest uncertainty at higher $\nu_{\mu}$ energies still comes from $\mathrm{K}^{+}$production, even including the SciBooNE measurement. . . 80

7.5 The fractional error for the BNB $\nu_{e}$ flux through MicroBooNE, broken up by systematic source. "Other" includes hadronic interactions, horn current un-

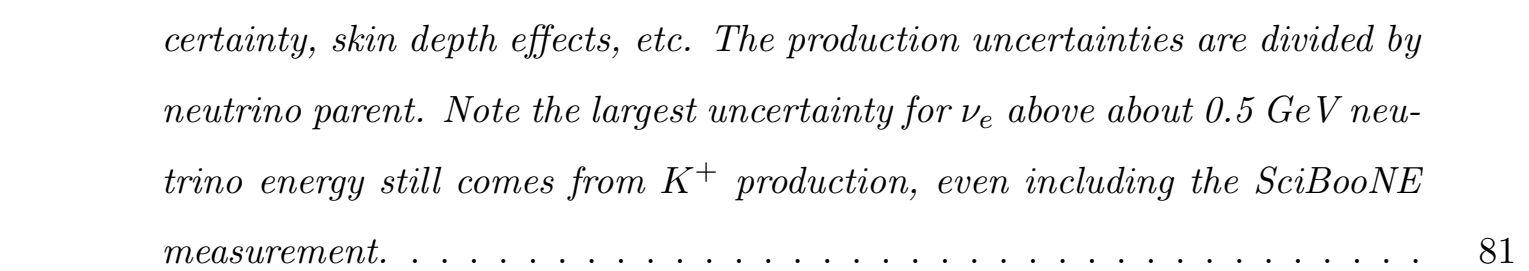


7.6 Muon neutrino charged-current cross section measurements and predictions as a function of neutrino energy. QE stands for quasielastic channels, RES

stands for resonant channels, and DIS stands for deep inelastic scattering

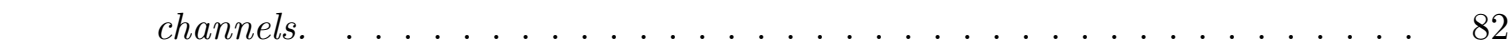

7.7 The fraction of $\nu_{\mu}$ energy imparted to the outgoing lepton $(\mu)$ in $\nu_{\mu}^{C C}$ inter-

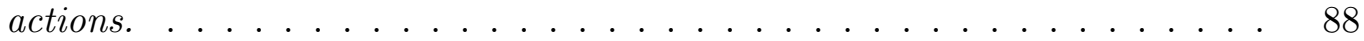

$7.8 \quad$ Reconstructed neutrino energy versus true neutrino energy for a sample of correctly identified $B N B \nu_{\mu}^{C C}$ interactions in the MicroBooNE TPC. . . . . 91

7.9 Distribution of signal (green) and backgrounds normalized for $0.5 \times 10^{20}$ protons-on-target worth of data, for reconstructed neutrino energy below 2.5 GeV. This comprises the sideband region. . . . . . . . . . . . . . . . . 92

7.10 Distribution of signal (green) and backgrounds normalized for $0.5 \times 10^{20}$ protons-on-target worth of data, for reconstructed neutrino energy between $2.5 \mathrm{GeV}$ and $5 \mathrm{GeV}$. This comprises the signal region, which has an $81 \%$ purity of $\nu_{\mu}^{C C}$ interactions from $K^{+}$decay in the beam. . . . . . . . . . . . . 93

7.11 A two-dimensional plot of energy versus angle for all kaons in the beam producing $\nu_{\mu}^{C C}$ interactions in the detector. . . . . . . . . . . . . . . 94

7.12 A two-dimensional plot of energy versus angle for the subset of kaons in Figure 7.11 which are reconstructed and selected for this analysis (having reconstructed neutrino energy above $2.5 \mathrm{GeV}) . \quad$. . . . . . . . . . . . . . 95

7.13 The kaon production angle distribution for all kaons in the beam producing $\nu_{\mu}^{C C}$ interactions in the detector (red), the subset of those which are reconstructed and selected in both the sideband and signal region (blue) and the subset of those in the Kaon enriched signal sample, with reconstructed neutrino energy above $2.5 \mathrm{GeV}$ (green). . . . . . . . . . . . . . . . . . . 96

7.14 The kaon production energy distribution for all kaons in the beam producing $\nu_{\mu}^{C C}$ interactions in the detector (red), the subset of those which are reconstructed and selected in both the sideband and signal region (blue) and the subset of those in the Kaon enriched signal sample, with reconstructed neutrino energy above $2.5 \mathrm{GeV}$ (green). . . . . . . . . . . . . . . . . . . 97 
7.15 The distribution of length of the longest track associated with the interaction contained within the fiducial volume for simulated backgrounds and signal (solid histograms) overlaid with data measurements ("on-beam" minus "off-beam" drawn in purple) for the sideband region in which reconstructed neutrino energy is less than $2.5 \mathrm{GeV}$. Statistical error bars are drawn on the data points, taking into account statistics from both the "on-beam" and "offbeam" samples. Below the main figure is a bin-by-bin ratio of data divided by simulation. . . . . . . . . . . . . . . . . . . . . . . . . . 99

7.16 The distribution of track multiplicity (number of tracks associated with the interaction) for simulated backgrounds and signal (solid histograms) overlaid with data measurements ("on-beam" minus "off-beam" drawn in purple) for the sideband region in which reconstructed neutrino energy is less than 2.5 GeV. Statistical error bars are drawn on the data points, taking into account statistics from both the "on-beam" and "off-beam" samples. Below the main figure is a bin-by-bin ratio of data divided by simulation. . . . . . . . . . . . . 100

7.17 The distribution of $\phi$ angle (measured in radians with respect to the vertical) of the longest track associated with the interaction for simulated backgrounds and signal (solid histograms) overlaid with data measurements ("on-beam" minus "off-beam" drawn in purple) for the sideband region in which reconstructed neutrino energy is less than $2.5 \mathrm{GeV}$. Statistical error bars are drawn on the data points, taking into account statistics from both the "on-beam" and "off-beam" samples. Below the main figure is a bin-by-bin ratio of data divided by simulation. . . . . . . . . . . . . . . . . . . . . . . . . . . . . 101 
7.18 The distribution of $\theta$ angle (measured in radians with respect to the beam direction) of the longest track associated with the interaction for simulated backgrounds and signal (solid histograms) overlaid with data measurements ("on-beam" minus "off-beam" drawn in purple) for the sideband region in which reconstructed neutrino energy is less than $2.5 \mathrm{GeV}$. Statistical error bars are drawn on the data points, taking into account statistics from both the "on-beam" and "off-beam" samples. Below the main figure is a bin-by-bin ratio of data divided by simulation. . . . . . . . . . . . . . . . . . . . . . . . 102

7.19 The distribution of multiple Coulomb scattering computed energy for the longest track associated with the interaction for simulated backgrounds and signal (solid histograms) overlaid with data measurements ("on-beam" minus "off-beam" drawn in purple) for the sideband region in which reconstructed neutrino energy is less than $2.5 \mathrm{GeV}$. Statistical error bars are drawn on the data points, taking into account statistics from both the "on-beam" and "offbeam" samples. Below the main figure is a bin-by-bin ratio of data divided by simulation. This plot has fewer entries than previous plots because only tracks longer than 1 meter have an associated MCS momentum as described in Section 7.3. . . . . . . . . . . . . . . . . . . . . 103

7.20 The distribution of reconstructed neutrino energy for simulated backgrounds and signal (solid histograms) overlaid with data measurements ("on-beam" minus "off-beam" drawn in purple) for the sideband region in which reconstructed neutrino energy is less than $2.5 \mathrm{GeV}$. Statistical error bars are drawn on the data points, taking into account statistics from both the "on-beam" and "off-beam" samples. Below the main figure is a bin-by-bin ratio of data divided by simulation. . . . . . . . . . . . . . . . . . . . . . . . . . . . 104 
7.21 The distribution of reconstructed neutrino energy for simulated backgrounds and signal (solid histograms) overlaid with data measurements ("on-beam" minus "off-beam" drawn in purple) for the higher-energy end of the sideband region in which reconstructed neutrino energy is between 1.5 and $2.5 \mathrm{GeV}$. Statistical error bars are drawn on the data points, taking into account statistics from both the "on-beam" and "off-beam" samples. Below the main figure is a bin-by-bin ratio of data divided by simulation. . . . . . . . . . . . . . . 105

7.22 The distribution of reconstructed neutrino energy for simulated backgrounds and signal (solid histograms) overlaid with data measurements ("on-beam" minus "off-beam" drawn in purple) for the signal region in which reconstructed neutrino energy is greater than $2.5 \mathrm{GeV}$. Statistical error bars are drawn on the data points, taking into account statistics from both the "onbeam" and "off-beam" samples. Below the main figure is a bin-by-bin ratio of data divided by simulation. . . . . . . . . . . . . . . . . . . . . . 106 


\section{List of Tables}

$3.1 \quad$ MicroBooNE LArTPC design parameters and nominal operating conditions. 14

4.1 A summary of the systematic uncertainties included in the MicroBooNE flux prediction. The dominant uncertainty is that from particle production. . . . 25

4.2 A summary of the systematic errors for the integrated flux, mostly from particle production. "Other" includes hadronic interactions, horn current uncertainty, and skin effect. . . . . . . . . . . . . . . . . . 25

6.1 A comparison of some of the important similarities and differences between the MiniBooNE detector and the MicroBooNE detector, which make the signal modeling in this sensitivity study difficult. . . . . . . . . . . . . . . . . . 40

6.2 The list of configurable parameters and their values used in the AlgoSingleE $\nu_{e}^{C C}$ signal selection algorithm. Note that for $\nu_{e}^{C C}$ interactions and "perfect reconstruction", these actual values the distance-based parameters represent are incredibly small (sub centimeter). The cut values are chosen to be much larger to simulate estimated automatic track and shower reconstruction resolutions. . . . . . . . . . . . . . . . 56

6.3 Event selection efficiencies on "perfect reconstruction". The right-most column indicates the final number of selected events including analysis cuts, normalized to the nominal MicroBooNE running period of $6.6 \times 10^{20}$ protons

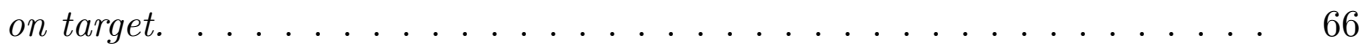

$6.4 \quad$ Summary of computed significances to the MiniBooNE low energy excess in MicroBooNE assuming an electron-like signal hypothesis. . . . . . . . . . . . 75 
7.1 A summary of the mean kaon production angle $\left(\overline{K_{\theta}}\right)$ and energy $\left(\overline{K_{E}}\right)$ in the $B N B$ for $\nu_{\mu}$ interactions (all interactions interacting within the TPC, and the subset of those which are selected in the analysis), and for $\nu_{e}$ interactions (providing backgrounds to the electron-like low energy excess search). Reported in brackets is the RMS of each distribution. . . . . . . . . . . . . . . 98 


\section{Acknowledgments}

This work would not have been possible without the help from a large number of humans, and one dog.

First, I would like to thank my advisor, Mike Shaevitz, for helping me every step along the way for the past six years. From selecting me from the undergraduate candidate pool for the summer REU internship at Nevis working on the Double Chooz experiment to serving as the sponsor for my thesis defense, Mike has always been there for me. The advisor-student relationship is an important one, and I am incredibly fortunate to have landed in Mike's group. During my tenure as a graduate student, I always felt supported and welcomed, and I never felt afraid to ask a question, no matter how stupid I worried it was.

I would like to thank perhaps the most influential teacher in my life, Hugon Karwowski. He taught me introductory mechanics and electromagnetism during my first year of undergraduate study at the University of North Carolina, and his passion for physics and creativity in creating test questions ("David Kaleko sits atop a frictionless ramp slanting down towards a pool filled with sharks. How long until he suffers a gruesome death?") is what truly spawned a love of physics within me. Throughout my TAing and private tutoring in graduate school at Columbia, I strove to teach my students in the same way as he taught me. His class was probably the only thing for which it was worth it to get up at 7 am.

I would also like to thank my closest coworkers in the Columbia group, including Kazuhiro Terao, David Caratelli, Georgia Karagiorgi, Leslie Camilleri, Vic Genty, Rachel Carr, and others I may have forgotten to include here. You provided me with the right 
combination of technical coding expertise and particle physics knowledge I needed to accomplish my goal of receiving my doctorate. It was a pleasure working along side each of you, and I am grateful to have shared the responsibility of driving the vans full of chatty summer students back and forth to Irvington each summer.

Additionally, I would like to thank the MicroBooNE collaboration as a whole. I enjoyed working with my coworkers in Wilson Hall at Fermilab; the opportunity to walk down the hall to ask your physics question to a world-leading expert on that topic is one I didn't take for granted. On the tenth floor, I found the friendly yet driven work environment to be a good fit for me. It was nice to take a break from coding to solve a Rubik's cube with a friend or talk shop over some Fermilab cafeteria food at lunch time. Moving from New York to Chicago to work at Fermilab full time was a decision I did not regret.

I would like to thank my family for their constant support outside of physics. My mother and father have always been there for me throughout my entire life, and their support continued during my (notably more curmudgeonly) years in graduate school.

Lastly, I would like to thank my girlfriend Carolyn for agreeing to quit her job and move to Chicago with me. Together we've made a home and a life together in Oak Park, and adopting our Labrador retriever puppy Rosie was the best decision we've ever made. Carolyn kept me sane throughout my time in graduate school. I wouldn't be where I am today without her. 


\section{Chapter 1}

\section{Introduction}

This thesis describes work towards the search for a low energy excess in MicroBooNE. What MicroBooNE is, what the low energy excess is, and how one searches for the latter in the former will be described in detail.

To begin, Chapter 2 will provide some introductory theoretical background about neutrinos both within and beyond the Standard Model of physics. Following that, Chapter 3 will describe the MicroBooNE detector, located at the Fermi National Accelerator Laboratory in Batavia, Il. This detector employs a liquid argon time projection chamber (LArTPC), a relatively new technology for neutrino detection, especially at the size of MicroBooNE (with order of meters drift distance). Next, Chapter 4 will describe the Booster Neutrino Beam (BNB), the beam of neutrinos produced at Fermilab by colliding primary protons with a beryllium target and focusing the outgoing charged secondaries. The relatively large uncertainties associated with the neutrino flux will be introduced, which are particularly important for the analyses described in this thesis. Chapter 5 goes on to describe in detail the excess of electron-like events seen in primarily $\nu_{\mu}$ beams, first by the LSND collaboration and then again by the MiniBooNE collaboration. Ultimately the MiniBooNE collaboration was unable to resolve whether the excess is of electron-like events, or photon-like events due to limitations of the detector technology. For this reason, the MicroBooNE experiment was proposed to measure the same neutrino beam at a similar location to MiniBooNE, but with the different LArTPC detector technology that has photon/electron discrimination 
capabilities.

Chapter 6 describes a simulation-based analysis done to estimate the sensitivity of the MicroBooNE detector to measure a MiniBooNE-like excess, with some assumptions about that excess. This is an important analysis to identify which sources of backgrounds are most relevant to this search in order to step closer to an eventual search for the excess in MicroBooNE data. As will be described, the dominant background to this search is the intrinsic $\nu_{e}$ contamination in the beam, about half of which come from $K^{+}$production at the BNB proton target. There is a relatively large flux uncertainty associated with with this $K^{+}$production, which is the subject of Chapter 7 .

Chapter 7 presents the first steps toward a $K^{+}$production at the BNB primary proton target measurement in MicroBooNE. This analysis selects and analyses high energy $\nu_{\mu}$ interactions in MicroBooNE, which provide a pure sample of $\nu_{\mu}$ from $K^{+}$decay. The in situ measurement of these interactions is used to constrain the aforementioned important intrinsic $\nu_{e}$ from $K^{+}$background for the low energy excess search.

In order to make the kaon production measurement, calculating the energy of several$\mathrm{GeV}$ muons from $\nu_{\mu}$ interactions in MicroBooNE is a necessary step. Given the muon kinematics in liquid argon, these particles travel on average many meters and almost always exit the active detector volume. Appendix A presents a publication (whose first author is the author of this thesis) describing in detail the multiple Couloumb scattering based method used to estimate the energy of muons which exit a LArTPC. The publication describes important discovery made about the underlying phenomenological formula which past LArTPC neutrino experiments have neglected: the formula needs to be re-tuned for use specifically in liquid argon.

The thesis concludes with a summary of the results of the three analyses described, along with the future prospects for those analyses and for the MicroBooNE experiment in general. 
CHAPTER 2. NEUTRINOS, NEUTRINO OSCILLATIONS, AND STERILE

NEUTRINOS

\section{Chapter 2}

\section{Neutrinos, Neutrino Oscillations, and Sterile Neutrinos}

\subsection{Introduction to Neutrinos}

In 1914, James Chadwick measured the energy spectrum of outgoing electrons in beta decay and showed that the spectrum was continuous 1], though it was expected to consist of one discrete, well defined value due to the presumed two-body nature of the decay. While some chose to believe that a lack of energy conservation is the underlying cause of the continuous spectrum, in 1930 Pauli proposed (translated from German) 22:

“...in the nuclei there could exist electrically neutral particles, which I will call neutrons, that have spin $1 / 2$ and obey the exclusion principal and that further differ from light quanta in that they do not travel with the velocity of light... The continuous beta spectrum would then make sense with the assumption that in beta decay, in addition to the electron, a neutron is emitted such that the sum of the energies of neutron and electron is constant."

Though Pauli named the particle a "neutron" (a name later used for the subatomic particle comprised of two down quarks and one up quark), his prediction was otherwise correct. Only 26 years later in 1956, the first neutrino was observed. 
Neutrinos make up three of the six leptons in the Standard Model of particle physics. There are three flavor eigenstates, each of which are paired to one of the other three leptons: electron neutrinos $\left(\nu_{e}\right)$, muon neutrinos $\left(\nu_{\mu}\right)$, and tau neutrinos $\left(\nu_{\tau}\right)$. Each neutrino has an antiparticle partner, the antineutrino. Neutrinos are particularly elusive because they are electrically neutral and only interact through the weak interaction and gravity. The number of active neutrino flavors is constrained to three $(2.984 \pm 0.0082)$ by precision measurements of the $Z$ decay width 3 .

The flavor of a neutrino is determined by measuring the outgoing lepton in charged current neutrino interactions. In such an interaction, a $\nu_{e}$ produces an electron, a $\nu_{\mu}$ produces a muon, and a $\nu_{\tau}$ produces a tau.

\subsection{Neutrino Oscillations}

It was until 1968 that neutrinos were widely believed to be massless. In this year, Ray Davis and collaborators were measuring neutrinos originating from within the sun ("solar neutrinos") by studying charged-current $\nu_{e}$ interactions on chlorine atoms. They measured only one third as many neutrino interactions as expected, a fact which wasn't fully understood until 2001. By combining results from other experiments (including from the Kamiokande experiment in Japan measuring a deficit of $\nu_{\mu}$ from cosmic ray interactions in the atmosphere in 1998 [4], and from the Sudbury Neutrino Observatory (SNO) experiment [5]), the idea that neutrinos oscillated from one flavor to another became widely accepted.

Neutrino oscillations occur when a neutrino produced as one flavor eigenstate changes to another flavor eigenstate after propagating a distance, $L$. Oscillation is only possible because the three mass eigenstates are non-zero and are different than the three flavor eigenstates. Neutrino propagation happens as a mixture of mass eigenstates with different DeBroglie wavelengths, which constructively and destructively interfere with one another. The mass eigenstates are referred to as $\nu_{1}, \nu_{2}$, and $\nu_{3}$ with masses $m_{1}, m_{2}$, and $m_{3}$ respec- 
tively. While the absolute masses are yet unknown, the mass-squared splitting between the masses have been measured $\left(\Delta m_{i, j}=m_{j}^{2}-m_{i}^{2}\right)$. This introduces two possible ordering configurations of the neutrino masses: the "normal" hierarchy in which $m_{1}<m_{2}<m_{3}$,

and the "inverted" hierarchy, in which $m_{3}<m_{1}<m_{2}$. The two hierarchy possibilities are depicted in Figure 2.1 [6]. As seen in the figure, the larger of the two mass splittings is referred to as $\Delta m_{\mathrm{atm}}^{2}$ and the smaller of the two is referred to $\Delta m_{\text {sol }}^{2}$ because they are generally measured with atmospheric neutrinos or solar neutrinos, respectively.

Neutrino Mass Hierarchy

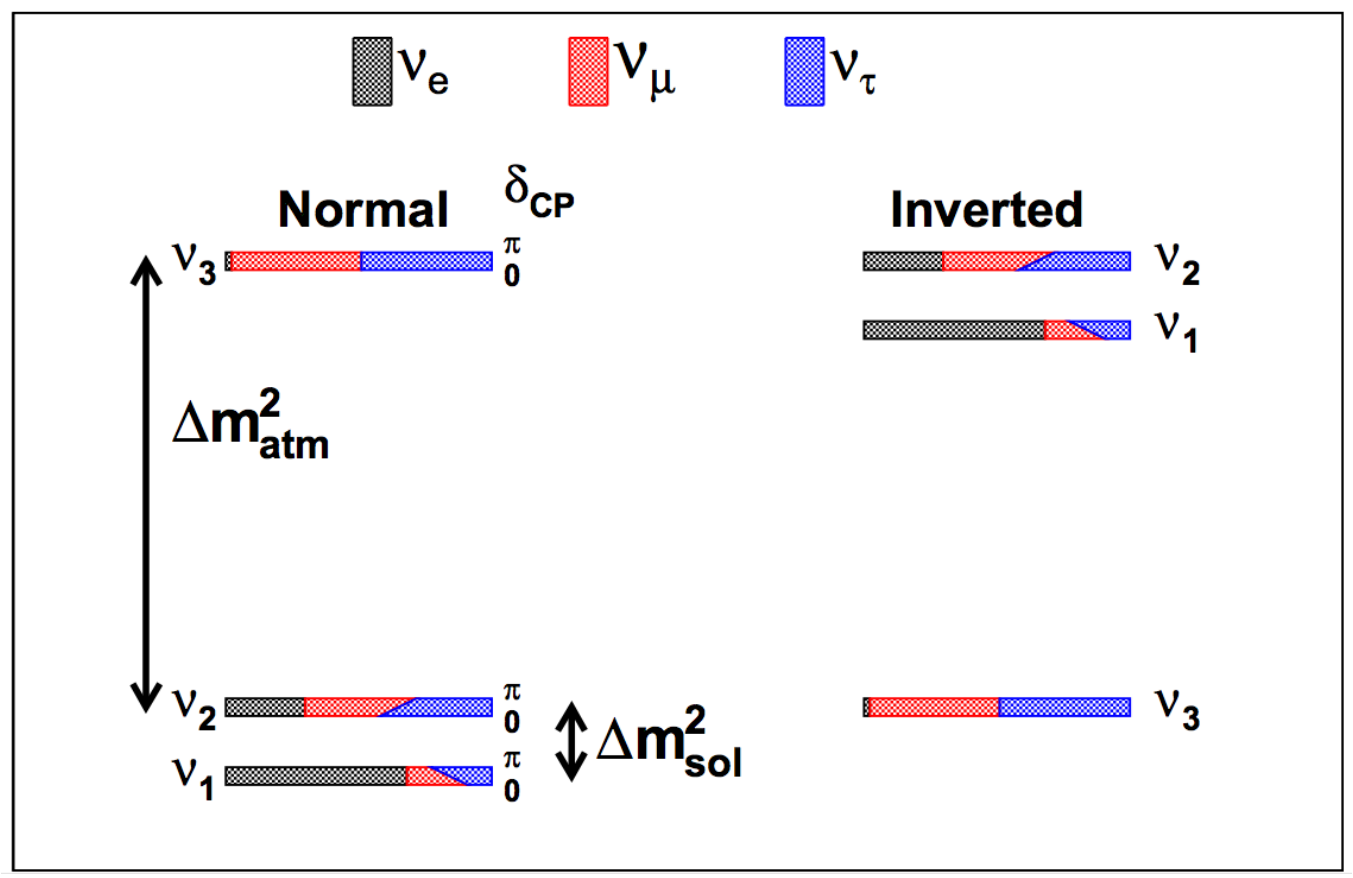

Figure 2.1: The neutrino mass hierarchy possibilities. The flavor eigenstate fraction for each mass eigenstate is shown by the relative amount of gray, red, or blue in each bar.

A neutrino mass eigenstate can be expressed as a quantum superposition of flavor eigenstates by way of the Pontecorvo-Maki-Nakagawa-Sakata (PMNS) $3 \times 3$ unitary mixing matrix, $U$ :

$$
\left|\nu_{i}\right\rangle=\sum_{\alpha}^{3} U_{i, \alpha}\left|\nu_{\alpha}\right\rangle
$$


where $\left|\nu_{i}\right\rangle$ represents the $i$ th mass eigenstate $(i=1,2,3)$ and $\left|\nu_{\alpha}\right\rangle$ represents the flavor eigenstate $(\alpha=e, \mu, \tau)$. Generally the PMNS matrix is factorized into three matrices parameterized by four parameters: three mixing angles $\theta_{12}, \theta_{23}, \theta_{13}$, and one $\mathrm{CP}$ violating phase $\delta$.

$$
U=\left[\begin{array}{ccc}
1 & 0 & 0 \\
0 & c\left(\theta_{23}\right) & s\left(\theta_{23}\right) \\
0 & -s\left(\theta_{23}\right) & c\left(\theta_{23}\right)
\end{array}\right]\left[\begin{array}{ccc}
c\left(\theta_{13}\right) & 0 & s\left(\theta_{13}\right) e^{-i \delta} \\
0 & 1 & 0 \\
-s\left(\theta_{13}\right) e^{-i \delta} & 0 & c\left(\theta_{13}\right)
\end{array}\right]\left[\begin{array}{ccc}
c\left(\theta_{12}\right) & s\left(\theta_{12}\right) & 0 \\
-s\left(\theta_{12}\right) & c\left(\theta_{12}\right) & 0 \\
0 & 0 & 1
\end{array}\right]
$$

where "c" stands for cos and "s" stands for sin.

In general, an experiment searching for neutrino oscillations has the choice of searching for appearance or disappearance. In appearance experiments, a neutrino of one known flavor is produced (flavor $\alpha$ ), and one attempts to measure that neutrino as another flavor (flavor $\beta$ ). Appearance searches have the added benefit of potentially shedding light on $\mathrm{CP}$ violation, which leads to appearance probabilities $P\left(\nu_{\alpha} \rightarrow \nu_{\beta}\right) \neq P\left(\bar{\nu}_{\alpha} \rightarrow \bar{\nu}_{\beta}\right)$. In a disappearance search, neutrinos of known flavor are produced at a known rate, and a decreased rate (deficit) of that same neutrino flavor is measured a distance away. Since $P\left(\nu_{\alpha} \rightarrow \nu_{\alpha}\right)=P\left(\bar{\nu}_{\alpha} \rightarrow \bar{\nu}_{\alpha}\right)$, disappearance searches allow for the combination of neutrino and anti-neutrino data for increased oscillation sensitivity.

A classic graduate-student quantum mechanical qualification exam question is to determine an oscillation probability assuming only two neutrinos. This can be parameterized with one effective mixing angle, $\theta$, and the nominal rotation matrix

$$
U=\left[\begin{array}{cc}
\cos \theta & \sin \theta \\
-\sin \theta & \cos \theta
\end{array}\right]
$$

A neutrino of flavor $\nu_{\alpha}$ will propagate as the superposition of the two mass eigenstates, $\nu_{1}$ and $\nu_{2}$

$$
\left|\nu_{\alpha}(t)\right\rangle=\cos (\theta) e^{-i E_{1} t}\left|\nu_{1}\right\rangle+\sin (\theta) e^{-i E_{2} t}\left|\nu_{2}\right\rangle
$$

Using the relativistic approximation (which is valid for neutrinos of almost any momenta, 
CHAPTER 2. NEUTRINOS, NEUTRINO OSCILLATIONS, AND STERILE

$p$, given their small mass, $m$ )

$$
E_{i}=p_{i}+\frac{m_{i}^{2}}{2 p_{i}}
$$

the oscillation probability for a neutrino of flavor $\alpha$ to be detected as flavor $\beta$ after traveling a distance $\mathrm{L}$ at approximately the speed of light is given by

$$
P_{\alpha \rightarrow \beta}=\left|\left\langle\nu_{\alpha}|| \nu_{\beta}\right\rangle\right|^{2}=\sin ^{2} 2 \theta \sin ^{2}\left(1.27 \frac{\Delta m^{2} L}{E}\right)
$$

where the 1.27 factor comes from a proper handling of units including $\hbar$ and $c$. The $L / E$ frequency modulation of oscillations is characteristic of two neutrino measurements, for example as beautifully demonstrated by the KamLAND collaboration measuring $\Delta m_{21}^{2}$ and $\theta_{12}$ with nuclear reactor anti-neutrinos, as shown in Figure 2.2 [7].

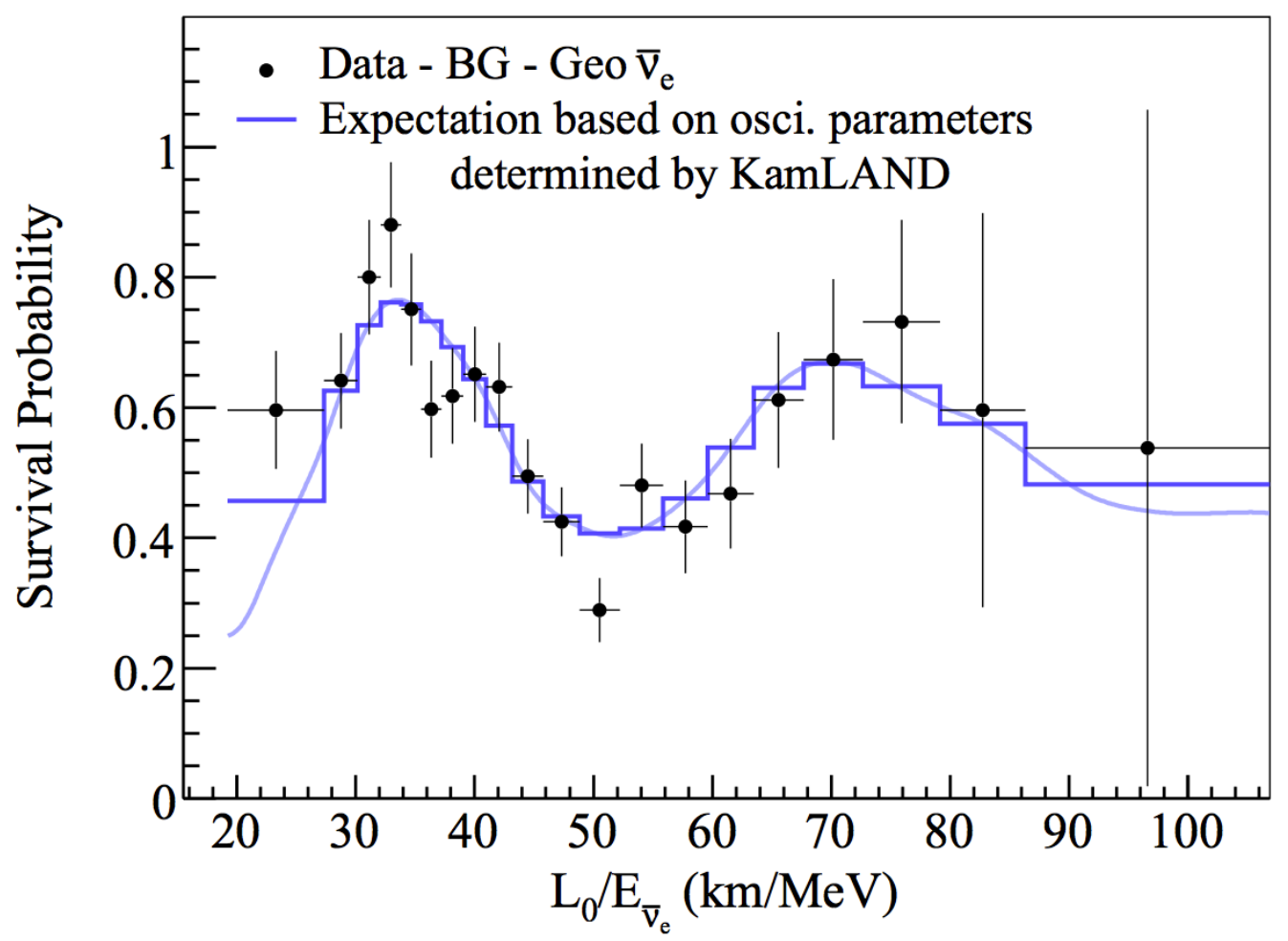

Figure 2.2: Results from the KamLAND collaboration measuring the survival probability of $\bar{\nu}_{e}$ from nuclear reactors. A clear oscillation as a function of $L / E$ as predicted by the two-neutrino model is shown. 


\subsection{Sterile Neutrinos}

While is is well known that there are only three active neutrino flavor states from the measured width of the $Z$ decay, it is possible to introduce one or more "sterile" neutrino states which do not couple weakly. Each addition of a sterile neutrino adds a row and column to the PMNS mixing matrix, and a new sterile mass eigenstate is defined as

$$
\left|\nu_{s}\right\rangle=\sum_{\alpha}^{3+N} U_{i, \alpha}\left|\nu_{\alpha}\right\rangle
$$

where $N$ is the number of additional sterile neutrinos added to the model.

Proposing the existence of one or more sterile neutrino eigenstates was inspired by the results of the LSND and MiniBooNE experiments, which are discussed in more detail in Chapter 5. LSND observed an excess of $\bar{\nu}_{e}$-like interactions in a $\bar{\nu}_{\mu}$ beam 8 . When fit to the three-neutrino oscillation model, the excess strongly disagreed with other measurements of neutrino mixing angles and $\Delta m^{2}$ values. The fit value of $\Delta m^{2}$ was on the order of 1 $e V^{2}$, orders of magnitude higher than previously measured values of $\Delta m_{12}^{2}$ and $\Delta m_{23}^{2}$. The existence of one or more sterile neutrinos could explain this drastically different measured $\Delta m^{2}$ value, since new additional mass eigenstate(s) are included in the propagating superposition, therefore changing neutrino oscillation probabilities. Including this measured sterile neutrino mass splitting in the "normal" or "inverted" hierarchy is shown in Figure $2.3[9]$.

Following the LSND experiment, the MiniBooNE experiment was proposed to search for oscillations with a similar mass splitting to that measured by LSND. In 2010, MiniBooNE observed a two anti-neutrino oscillation appearance-only measurement consistent with LSND over the null oscillation hypothesis at the $98 \%$ confidence level.

The following chapter of this thesis provides an introduction to the MicroBooNE detector, which was proposed to search for the same MiniBooNE excess but with a different detector technology. 
CHAPTER 2. NEUTRINOS, NEUTRINO OSCILLATIONS, AND STERILE NEUTRINOS

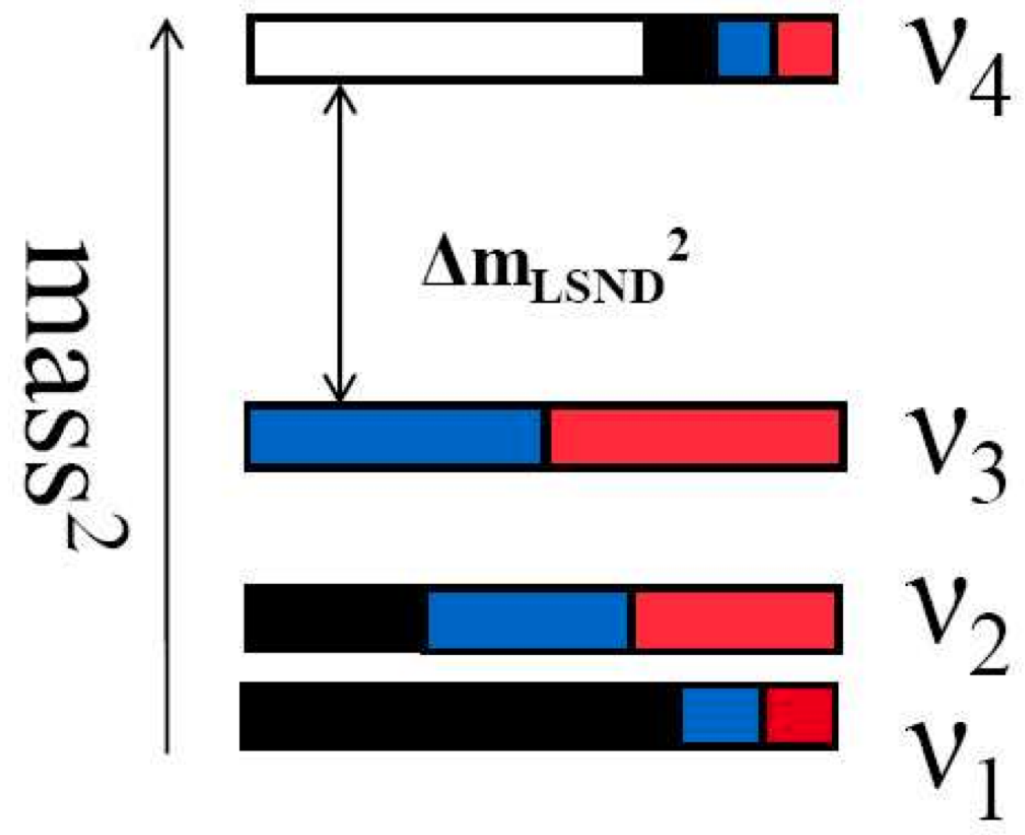

Figure 2.3: Mass hierarchy with one heavy sterile neutrino included. The $\delta m^{2}$ from LSND is incorporated. 


\section{Chapter 3}

\section{The MicroBooNE Detector}

The purpose of this chapter is to explain the technical details of the MicroBooNE detector. An understanding of how a liquid argon time projection chamber like MicroBooNE works is crucial for understanding the results of analyses described in later chapters. Using this specific detector technology gives rise to certain backgrounds in measurements which are relevant to MicroBooNE that may not be relevant for other experiments using different detection techniques, like MiniBooNE. Additionally, understanding how the detector works sheds light on what detector-specific uncertainties are present in MicroBooNE analyses.

\subsection{Introduction}

The MicroBooNE (the Micro Booster Neutrino Experiment) detector 10 is a $\sim 60$ ton fiducial mass (170 ton total mass) liquid argon time projection chamber (LArTPC) contained within a cylindrical cryostat, located on-axis of the Booster Neutrino Beam-line (BNB) 470 meters downstream from the neutrino production target at the Fermi National Accelerator Laboratory (FNAL) in Batavia, Illinois. A schematic of how a LArTPC works is shown in Figure 3.1. A LArTPC involves a detector medium in an external electric field. Particles traversing the medium both create scintillation light, which are observed by photomultiplier tubes (PMTs, not shown in Figure 3.1) and leave trails of ionization electrons. These ionization electrons are drifted by the electric field past a number of closely spaced wire planes at different pitch angles. The signals generated in each plane as the electrons either pass 
close to wires or are collected on wires are what are used to create a two-dimensional image of the event. Combining information from multiple planes along with that from the PMTs allows for the creation of three-dimensional reconstructions of the event. Additionally, the relative size of the signals on the wire planes provide calorimetric information, which is used for particle identification capabilities.

The main components of the MicroBooNE TPC (the TPC, the light collection system, and the readout and triggering system) are described in the following sections.

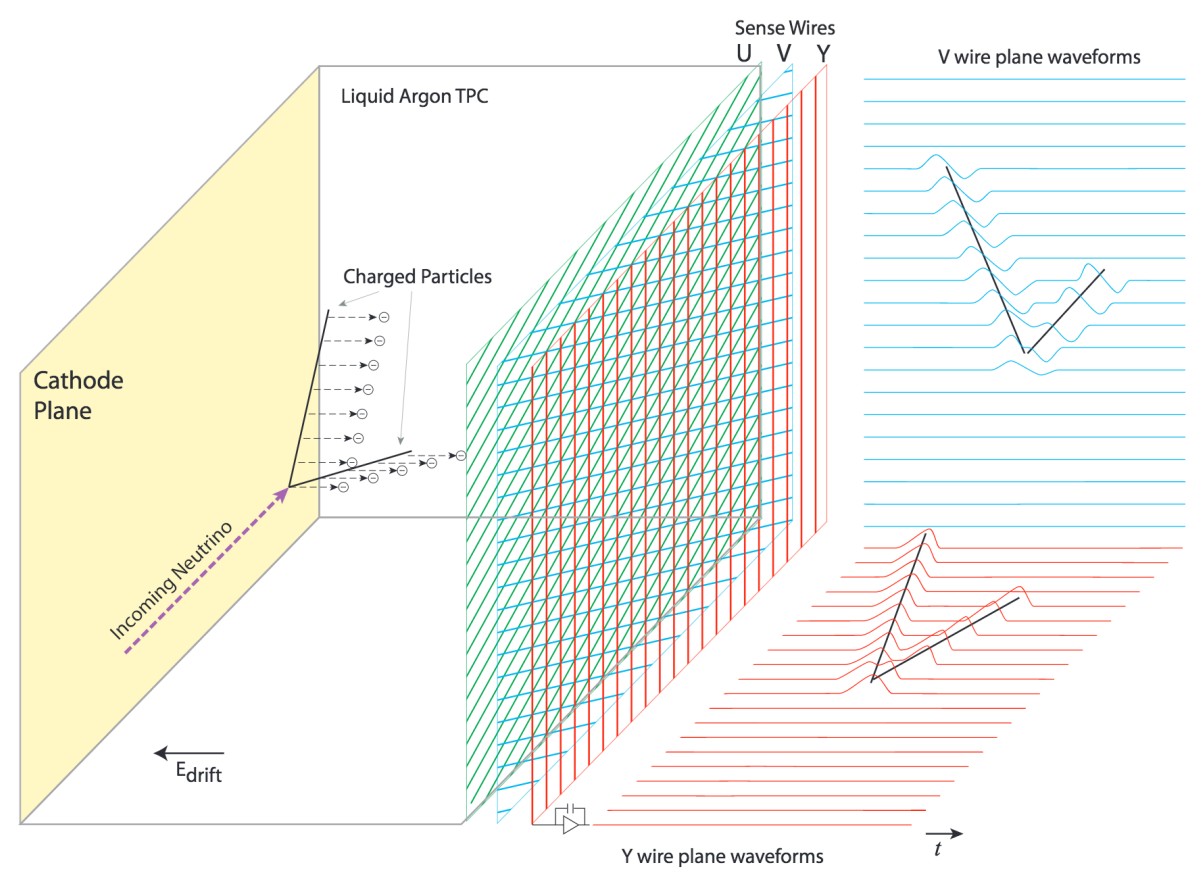

Figure 3.1: A cartoon schematic of how a LArTPC works. Ionization electrons from particles traversing the detector medium are drifted by an electric field, $E_{\text {drift }}$ past multiple planes of sense wires. The signals on those wires create several two-dimensional images of the event, which are combined to create a three-dimensional reconstruction of the event. Note that in a LArTPC, PMTs are used to collect scintillation light, but are not drawn in this diagram. 


\subsection{Time Projection Chamber}

The time projection chamber (TPC) used in the MicroBooNE experiment is a rectangular prism with dimensions $2.3 \mathrm{~m}$ vertical $\times 2.6 \mathrm{~m}$ horizontal $\times 10.4 \mathrm{~m}$ length (along the beam direction). The 8256 stainless steel sense wires forming the anode planes have a plane-toplane spacing of $3 \mathrm{~mm}$, and the wires on each plane are separated with a $3 \mathrm{~mm}$ wire pitch. The wires are connected to application-specific integrated circuits (ASICs) which operate at liquid argon temperatures. There are three wire planes. The first two planes (from the point of view of drifting electrons) each consist of 2400 wires and are induction planes, at angles \pm 60 degrees relative to the vertical. The third wire plane consists of 3456 wires and is a collection plane, with vertically oriented wires. The electric field is created by a series of $642.54 \mathrm{~cm}$ diameter stainless steel pipes shaped into a rectangular loop held in place by a frame built of G10, forming the field cage. The negatively charged cathode is held at a high voltage (operating voltage is $70 \mathrm{kV}$ ), and this voltage is incrementally stepped down across the field cage tubes with a voltage divider chain, with an equivalent resistance of $250 \mathrm{M} \Omega$ between each tube. The distance from center-to-center of adjacent field cage loops is $4 \mathrm{~cm}$. This creates a uniform electric field within the LArTPC. A 3D rendering of the MicroBooNE TPC within the cryostat is shown in Figure 3.2. A summary of the MicroBooNE LArTPC design parameters and nominal operating conditions are described in Table 3.1 10.

\subsection{Light Collection System}

An important ingredient to the ultimate 3D reconstruction of particle interactions within a LArTPC is the light collection system. While the wire signals alone suffice to reconstruct $3 \mathrm{D}$ interactions, the absolute timing of the event (referred to as $t_{0}$ ) is unknown so there is ambiguity in the drift direction. Since the time scale with which scintillation light is created and propagates (nanoseconds) is orders of magnitude faster than that with which ionization electrons drift (milliseconds), measuring this light allows to clarify this ambiguity to high precision. Additionally, the scintillation light from interactions is relatively localized, and therefore combining the measured PMT signals with the physical position of the 


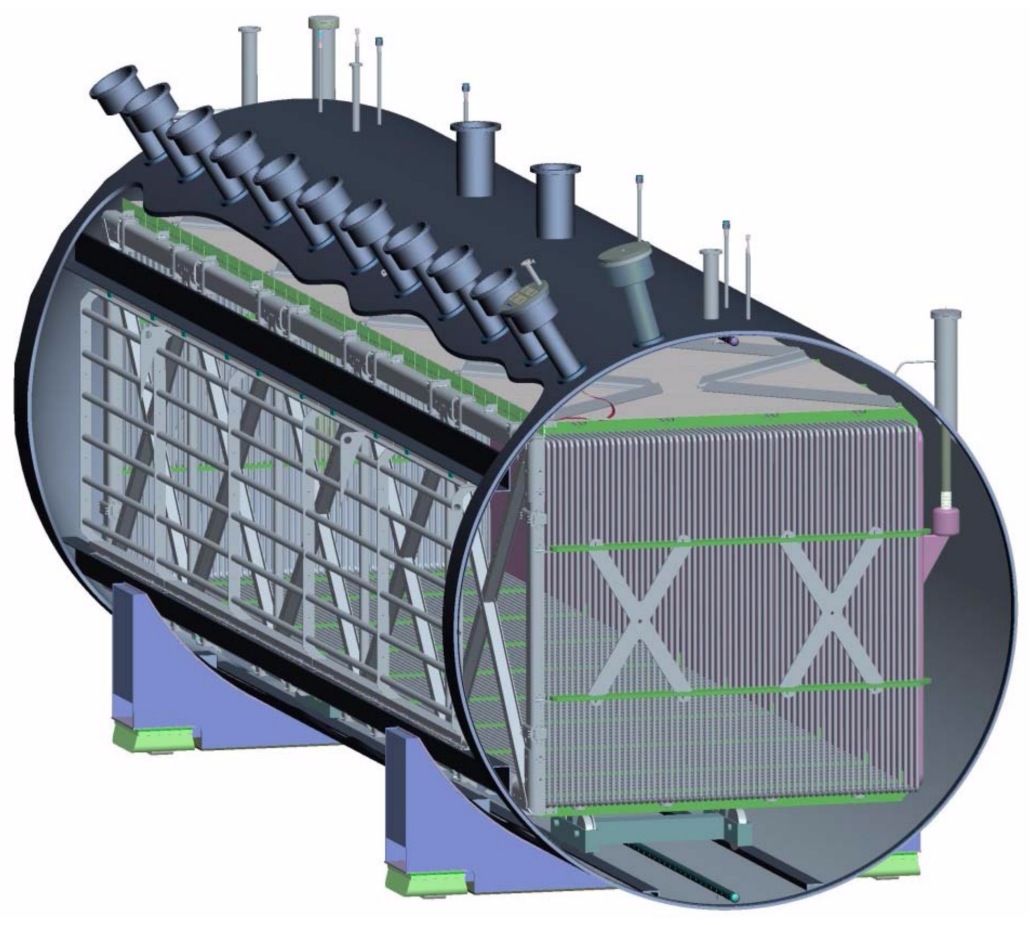

Figure 3.2: A 3D rendering of the MicroBooNE detector. The rectangular time projection chamber (TPC) fits within the cylindrical cryostat. The feedthroughs along the top allow for the PMT and sense wire signals to be read out to the DAQ. Not shown are the photomultiplier tubes (PMTS) located on the wall behind the sense wire planes.

signal allows to match individual flashes of light with different interactions, which may have different $t_{0} \mathrm{~s}$. This is important to help tag and reject cosmic-induced backgrounds which may arrive outside of the expected beam neutrino arrival times.

The light collection system in MicroBooNE consists of 32 8-inch diameter Hamamatsu R5912-02mod cryogenic PMTs. These PMTs are mounted in a plane behind the three sense wire planes. The physical location of these PMTs is shown in Figure 3.3. These 32 PMTs provide $0.85 \%$ photocathode coverage. Each PMT has mounted in front of it an acrylic plate, as shown in Figure 3.4. This plate is coated with TPB, an organic fluor which serves as a wavelength-shifting material. TPB absorbs the VUV scintillation light photons (128 $\mathrm{nm}$ wavelength in liquid argon) and re-emits it at visible wavelengths detectable by the 
Table 3.1: MicroBooNE LArTPC design parameters and nominal operating conditions.

\begin{tabular}{|c|c|}
\hline Parameter & Value \\
\hline \# Anode planes & 3 \\
\hline Anode planes spacing & $3 \mathrm{~mm}$ \\
\hline Wire pitch & $3 \mathrm{~mm}$ \\
\hline Wire type & SS, diam. $150 \mu \mathrm{m}$ \\
\hline Wire coating & $2 \mu \mathrm{m} \mathrm{Cu}, 0.1 \mu \mathrm{m} \mathrm{Ag}$ \\
\hline Design Wire tension & $6.9 \mathrm{~N} \pm 1.0 \mathrm{~N}$ \\
\hline \# wires (total) & 8256 \\
\hline \# Induction plane (U) wires & 2400 \\
\hline \# Induction plane $(\mathrm{V})$ wires & 2400 \\
\hline \# Collection plane (Y) wires & 3456 \\
\hline Wire orientation (w.r.t. vertical) & $+60^{\circ},-60^{\circ}, 0^{\circ}(\mathrm{U}, \mathrm{V}, \mathrm{Y})$ \\
\hline Cathode voltage (nominal) & $-128 \mathrm{kV}$ \\
\hline Bias voltages $(\mathrm{U}, \mathrm{V}, \mathrm{Y})$ & $-200 \mathrm{~V}, 0 \mathrm{~V},+440 \mathrm{~V}$ \\
\hline Drift-field & $500 \mathrm{~V} / \mathrm{cm}$ \\
\hline Max. Drift Time, Cathode to U (at $500 \mathrm{~V} / \mathrm{cm}$ ) & $1.6 \mathrm{~ms}$ \\
\hline \# Field-cage steps & 64 \\
\hline Ring-to-ring voltage step & $2.0 \mathrm{kV}$ \\
\hline
\end{tabular}

PMTs, peaked at $425 \mathrm{~nm}$.

\subsection{Electronics, Readout, and Triggering}

Both of the two main subsystems of the MicroBooNE LArTPC (the TPC sense-wire planes and the optical PMTs) create analog signals which must be read out and digitized for use in analyses. This process involves amplification and shaping of the signals, and ultimately ends with the data acquisition (DAQ) software writing the digitized data to disk. While the readout is designed to have an additional data stream that continually writes to disk (designed with the hopes of measuring neutrino interactions from a potential future super- 


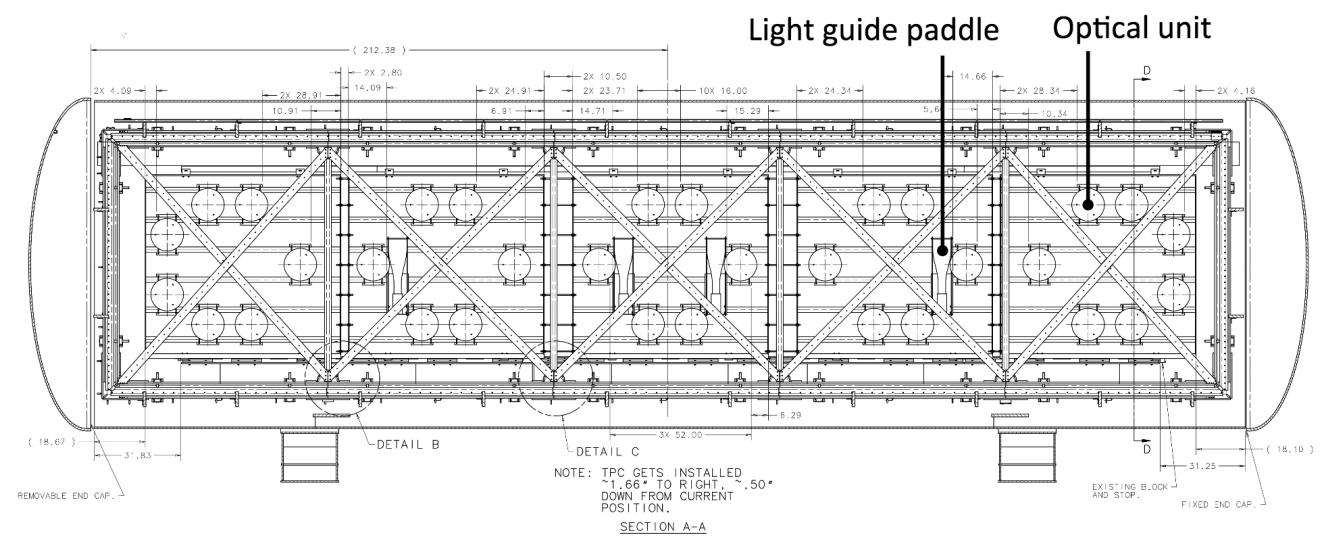

Figure 3.3: A side-on view of the MicroBooNE detector showing the location of the 32 PMTs (labeled "optical units") and the four light guide paddles.

nova explosion), the primary data stream reads out and stores signals only for a brief period of time when a hardware trigger is issued. The specifics of the readout and triggering for MicroBooNE are discussed in this section.

A schematic overview of the TPC and PMT signal processing and readout stages is shown in Figure 3.5. The analog signals from the 8256 sense wires in the TPC pass through CMOS analog front end ASICs which operate on cold motherboards at liquid argon temperatures. The signals are then shaped and amplified by cold intermediate amplifiers before passing through a warm feed-through. The signals are received by custom-designed LArTPC readout modules distributed over nine readout creates, which digitize the signals and process them. The TPC wire signals are digitized at $16 \mathrm{MHz}$ and then down-sampled in the digitization process to $2 \mathrm{MHz}$. The TPC system reads out four $1.6 \mathrm{~ms}$ frames of wire signal data associated with one event. This time is chosen based on how long it takes ionization electrons from the cathode side of the TPC to drift to the anode wires (roughly $1.6 \mathrm{~ms}$ depending on drift field). Reading out one frame before a trigger is issued, along with two frames after ensures enough data is available to identify both a neutrino interaction, as well as all cosmic ray signals that arrive soon enough before or after the neutrino which need to be reconstructed in analyses. 


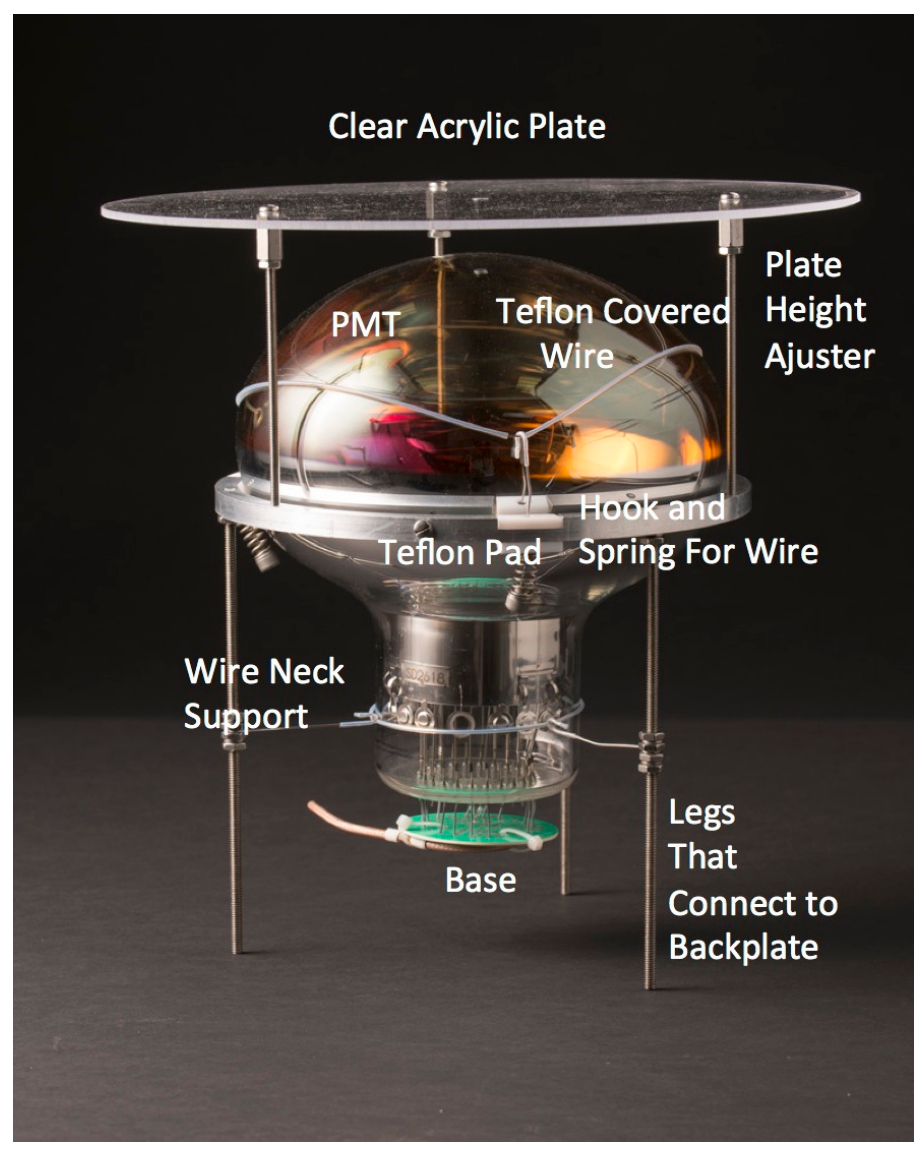

Figure 3.4: A picture of one of the 8 inch Hamamatsu R5912-02mod cryogenic photomultiplier tubes (PMT) used in the MicroBooNE detector. Note the clear acrylic plate, which is coated with a wavelength-shifting organic fluor (TPB) before installation to convert the VUV liquid argon scintillation light into the visible spectrum, detectable by the PMT.

A similar process occurs for the PMT signals. The PMT signals undergo separate shaping with a $60 \mathrm{~ns}$ peaking time to allow for digitization of several samples on the rising edge of a signal for more precise timing reconstruction abilities. The PMT signals are digitized at $64 \mathrm{MHz}$, but are not read out continuously during the same $4 \times 1.6 \mathrm{~ms}$ TPC readout time; only shaped PMT signals above a small discriminator threshold are read out and stored for later reconstruction. The PMT signals are split into high- and low- gain channels, to extend the dynamic range of the ADC.

The readout of both TPC and PMT systems are initiated by triggers formed in a separate 


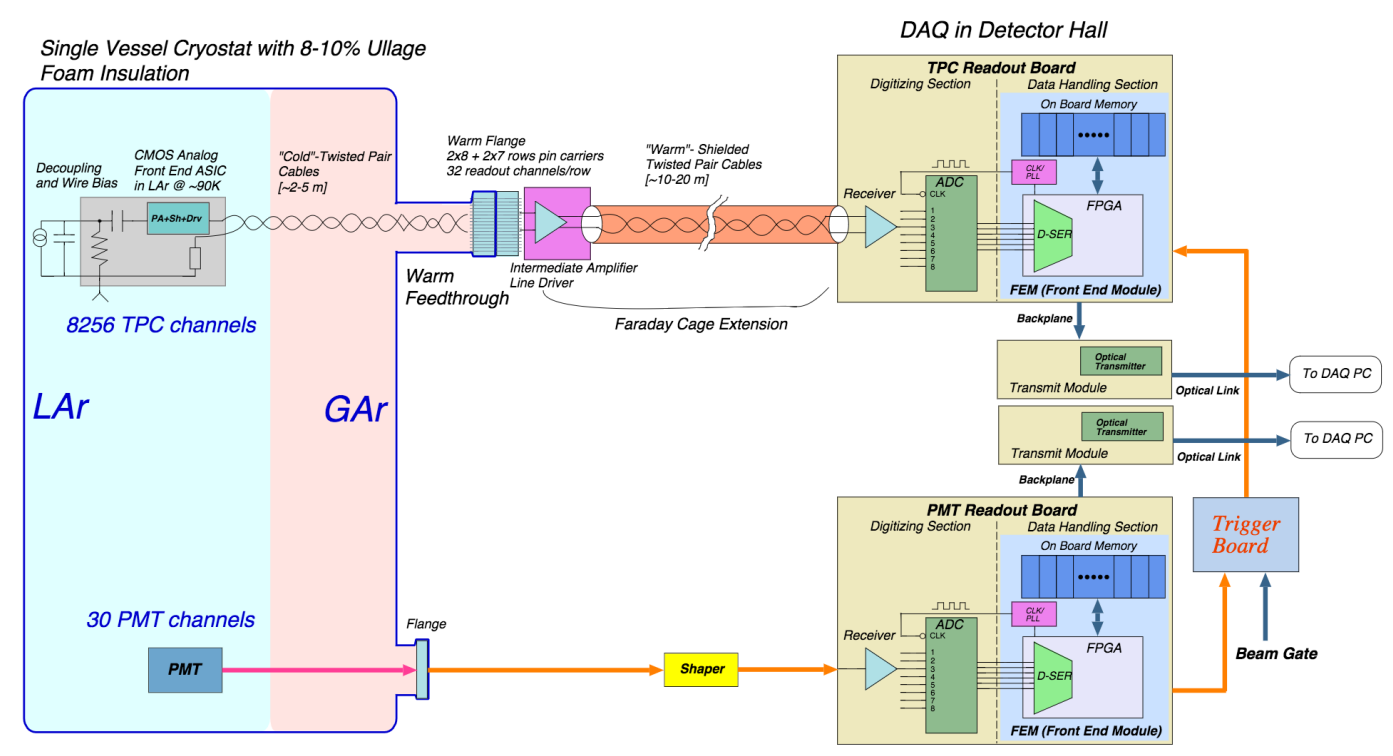

Figure 3.5: The MicroBooNE readout schematic. On the left are portions of the readout operating at argon temperatures. Signals pass through feedthroughs into warm electronics readout boards, unique for the TPC (sense wire signals) and the light collection system (PMT signals). These signals are combined with external timing signals from the accelerator to form triggers that initiate readout of all systems.

trigger board located in a warm electronics readout crate. While many different triggers are used by MicroBooNE, the one relevant for this analysis is the BNB trigger. To form this trigger, a timing signal from the BNB accelerator is shaped and fed into the trigger board. The FPGA firmware in the PMT front end readout modules generates a PMT trigger when PMT signal multiplicity is greater than 1 and summed PMT pulse-height is more than 2 photo-electrons summed over all of the high gain PMT channels. If a PMT trigger is issued by the firmware in coincidence with the $1.6 \mu \mathrm{s}$ beam gate window from the accelerator, a BNB trigger is generated by the trigger board. This trigger signal is fanned-out to all readout crates (TPC and PMT), instructing them to initiate a readout simultaneously. Once the readout is complete, the data from each readout crate is packaged and shipped to the DAQ software, which assembles all of the data into one event in memory, which is saved to disk and eventually used in reconstruction and analysis. Note that ultimately, 
the MicroBooNE collaboration has moved towards using a software- based trigger for its beam-based analyses. 


\section{Chapter 4}

\section{The Booster Neutrino Beam}

The purpose of this chapter is to describe how neutrinos are produced in the Booster Neutrino Beam-line (BNB) at the Fermi National Accelerator Laboratory. An understanding of how these neutrinos are produced and their flux through the MicroBooNE detector is necessary to properly interpret the results of the low energy excess analysis and kaon production analysis, described in Chapters 5 and 6 . In describing the neutrino production techniques, the reader will be introduced to the sources of systematic uncertainties associated with the neutrino production, both in terms of how they arise, and their magnitude.

\subsection{The Booster Neutrino Beam}

The Booster Neutrino Beam-line (BNB) collides protons at $8.89 \mathrm{GeV} / \mathrm{c}$ momentum from the Fermilab Booster synchrotron with a beryllium target to produce a high flux of neutrinos. The layout of the BNB is shown in Figure 4.1 11, and the relevant steps of the neutrino production process will be described in the following sections.

\subsubsection{Primary Proton Beam}

The protons originate from $H_{2}$ gas molecules converted to $H^{-}$ions via a Cockroft-Walton generator, and are initially accelerated to approximately $1 \mathrm{MeV}$ kinetic energy. These ions are subjected to a linear accelerator using alternating electromagnetic fields to increase their energy to about $400 \mathrm{MeV}$. Passing through a carbon foil removes electrons, and the 


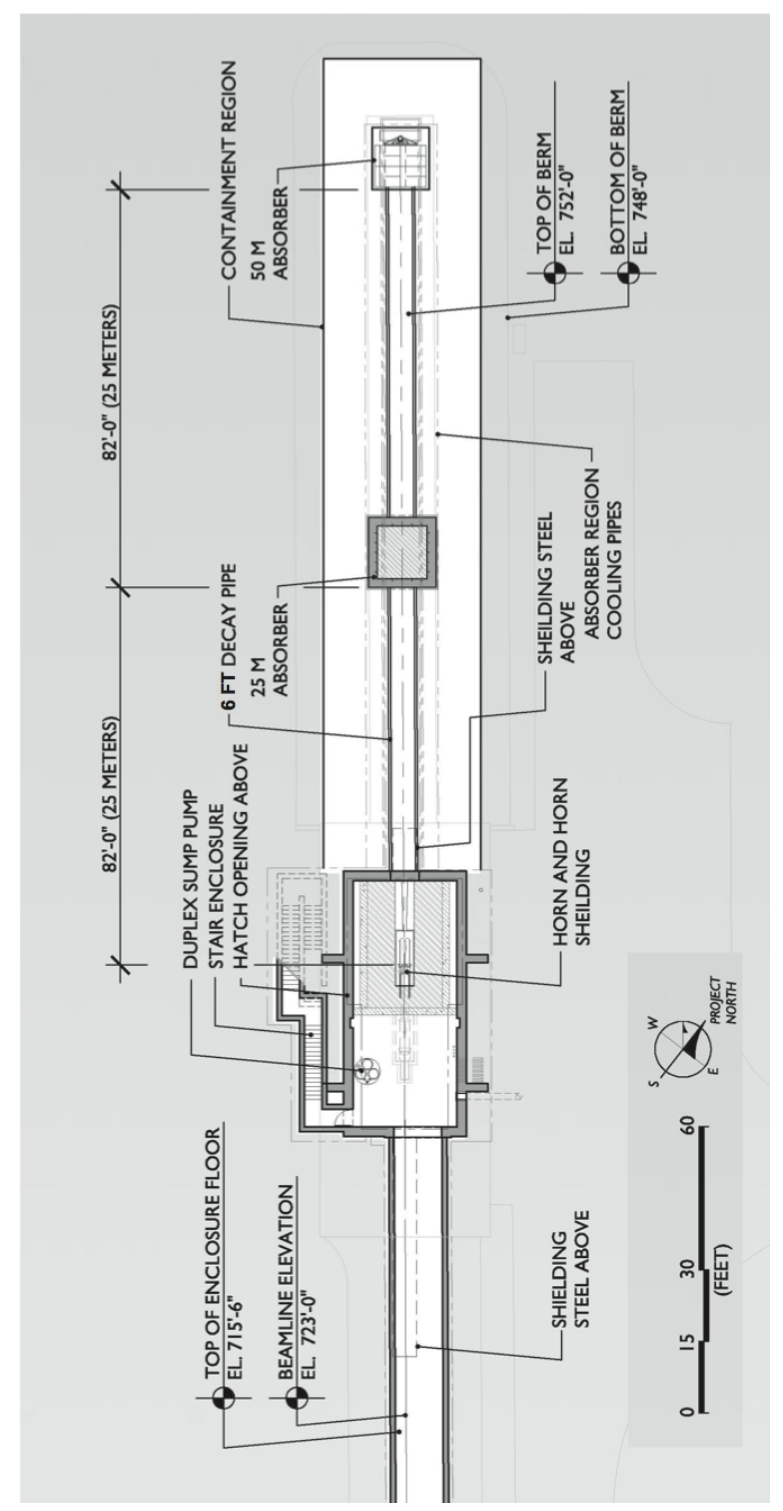

Figure 4.1: Overall layout of the BNB. The primary proton beam, extracted from the Booster, enters the target hall from the left. Upon exiting the target hall, particles encounter a 50meter-long decay region, terminating in the beam stop on the right.

bare protons enter the Booster synchrotron where they are accelerated up to $8.89 \mathrm{GeV} / \mathrm{c}$ momentum. The protons are bunched in "beam spills" containing roughly $4 \times 10^{12}$ protons spaced throughout a $1.6 \mu \mathrm{s}$ time window per spill. The protons are then directed towards a thick beryllium target. 
The absolute number of protons directed on target (POT) is measured by two toroids upstream of the target which are part of a larger beam monitoring system. The error on the POT is on the order of $2 \%$. Additional beam characteristics are monitored by beam position monitors (BPMs), a multi-wire chamber, and a resistive wall monitor (RWM) which together measure beam intensity, timing, width, position, and direction.

\subsubsection{Proton Target and Focusing Horn}

The beryllium target is $71.1 \mathrm{~cm}$ long, which corresponds to 1.7 proton interaction lengths, and is $0.51 \mathrm{~cm}$ in radius. Beryllium is chosen as the proton target because its relatively low Z (4) minimizes radiative losses from the protons before their p-Be interactions which produce secondary mesons $\left(\pi^{ \pm}, K^{ \pm}, K_{L}^{0}\right)$.

The beryllium target is located within a larger focusing electromagnet, referred to as the horn. A schematic drawing of the horn is shown in Figure 4.2. The horn is an aluminum alloy pulsed toroidal electromagnet. The pulsed current has a peak at $170 \mathrm{kA}$ and a time-width of $143 \mu \mathrm{s}$, coincident with the proton beam arrival time on the target. The current flows along the inner conductor, then returns along the outer conductor. The magnetic fields created by this current have a maximum field value of 1.5 Tesla and fall off as $1 / R$ from the cylindrically symmetric axis of the horn. These fields serve to focus the

charged secondaries produced in the p-Be interactions. The direction of the current can be switched to focus the positively charged secondaries, or the negatively charged secondaries, ultimately producing a beam of primarily neutrinos ("neutrino mode") or of primarily antineutrinos ("antineutrino mode") respectively.

Downstream of the horn is a concrete collimator $(214 \mathrm{~cm}$ long, between $30 \mathrm{~cm}$ and 35.5 $\mathrm{cm}$ in radius) which absorbs particles that would not otherwise contribute to the neutrino flux. Following the collimator is a 45 meter long ( 1 meter radius) air-filled cylindrical decay region, ending in a beam-stop made of steel and concrete which contains an array of gas proportional counters to detect muons penetrating the beam-stop. Also about half way 


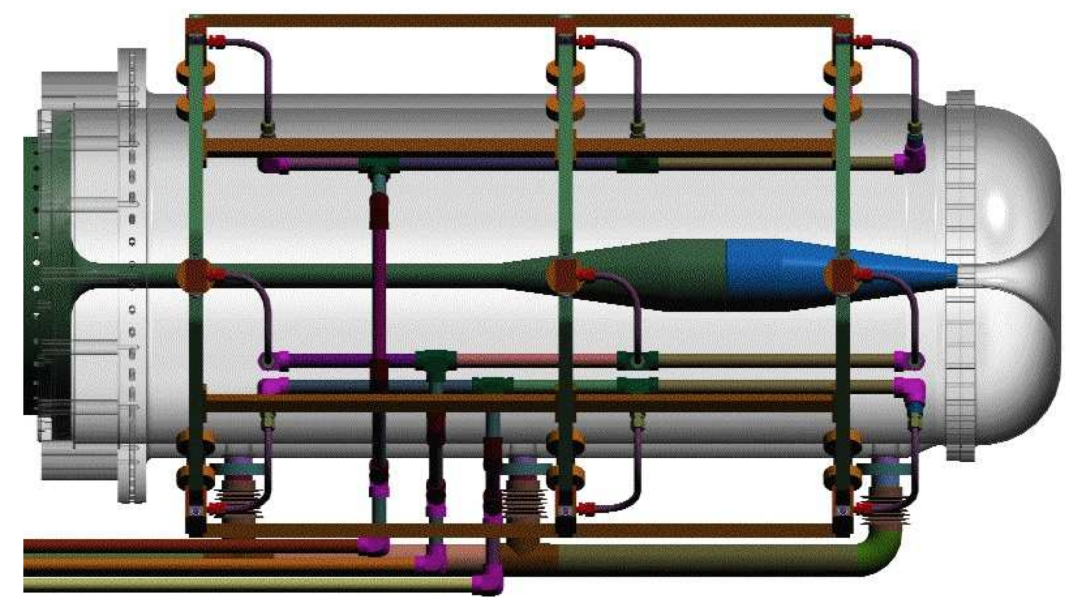

Figure 4.2: The BNB focusing horn system. The gray outer conductor is drawn transparent for visualization purposes. The beryllium target lies within the central hollow tube axis. A current flows along the inner conductor, returning along the outer conductor.

through the length of the decay region is an absorber consisting of ten removable steel plates for systematic studies, which were not used in the analyses described in this thesis. A schematic depicting the proton beam interacting with the beryllium target within the focusing horn is shown in Figure 4.3 .

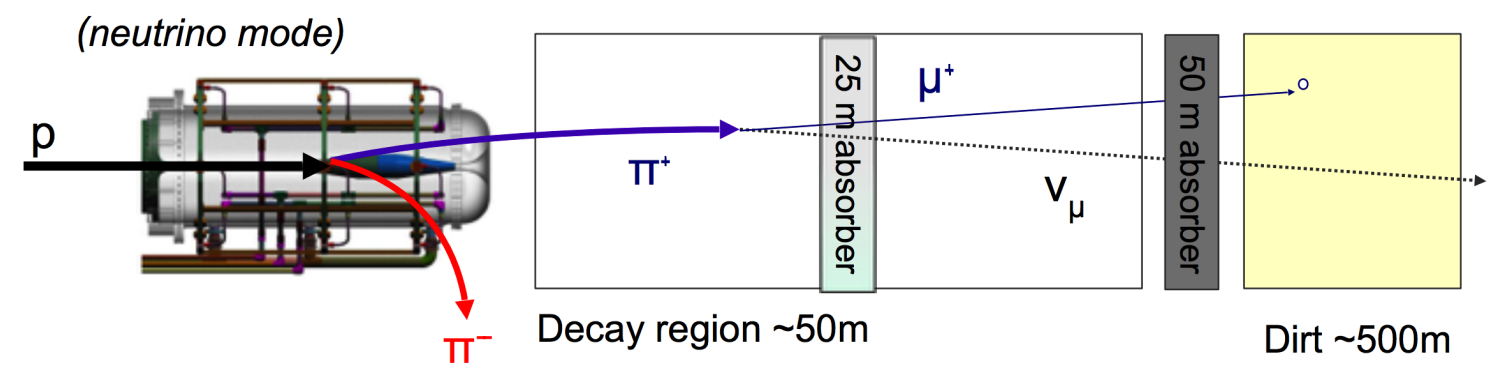

Figure 4.3: A cartoon diagram of the incident $8.89 \mathrm{GeV} / \mathrm{c}$ proton beam (from the left) colliding with the beryllium target within the focusing horn. Shown is the current configuration for the horn referred to as "neutrino mode" in which positive charged secondaries are focused into the decay region. The $25 \mathrm{~m}$ absorber drawn is removable, and was not used for the analyses described in this thesis. 


\subsection{Monte Carlo Neutrino Flux Prediction}

The neutrino flux through MicroBooNE is determined using a Geant4 12] Monte Carlo simulation of the beam-line, focusing horn, and decay region. The beam-line geometry modeled in Geant4 includes the position and material composition of all components of the $\mathrm{BNB}$, through which the primary protons and all other particles propagate. The primary protons are simulated with the expected beam optics properties upstream of the target. The primary p-BE interactions are simulated using custom tables for the production of outgoing particles including protons, neutrons, $\pi^{ \pm}, K^{ \pm}$, and $K^{0}$ created from production models based on external data. The reason custom tables are used is that the variation in Geant4 hadron-production models is large.

The custom physics model using external data to simulate the production of secondary mesons in the primary p-Be interactions is described in detail in Ref [11 but will be summarized here. The custom tables describe the double differential cross section for the production of each secondary species as a function of the proton transverse and longitudinal momentum components. Existing pion and kaon production data from the HARP [13, E910 [14], and several other production experiments are used in many of the Sanford and Wang parameterization fits in the parameter space relevant to MicroBooNE [15]. The fitting parametrization is described with 9 Sanford-Wang model parameters $\left(c_{1}\right.$ to $\left.c_{9}\right)$ :

$$
\frac{d^{2} \sigma}{d p d \Omega}=c_{1} p^{c_{2}}\left(1-\frac{p}{p_{B}-c_{9}}\right) \exp \left[-c_{3} \frac{p^{c_{4}}}{p_{B}^{c_{5}}}-c_{6} \theta\left(p-c_{7} p_{B} \cos ^{c_{8}} \theta\right)\right]
$$

where $p$ and $\theta$ are the momentum and angle of the outgoing secondary mesons.

Since no measurements for $K^{+}$production exist at the $8.89 \mathrm{GeV} / \mathrm{c}$ BNB primary proton momentum, the Feynman scaling hypothesis is used to extrapolate from $K^{+}$production measurements at different primary proton momenta. The Feynman scaling model function depends only on the transverse proton momentum $p_{T}$ and the Feynman scaling factor $x_{F}$, which is the ratio of the longitudinal momentum, $p_{L}^{C M}$, to the maximum longitudinal momentum, $p_{L}^{C M(\max )}$,

$$
x_{F}=\frac{p_{L}^{C M}}{p_{L}^{C M(\max )}} .
$$


According to the Feynman scaling model, the invariant cross section can be written in terms of seven parameters $\left(c_{1}\right.$ to $\left.c_{7}\right)$ as

$$
E \frac{d^{3} \sigma}{d p^{3}}=c_{1} \exp \left[-c_{2} p_{T}-c_{3}\left|x_{F}\right|^{c_{4}}-c_{5} p_{T}^{2}-c_{7}\left|p_{T} \dot{x}_{F}\right|^{c_{6}}\right]
$$

While this parameterization represents the measurements well, the uncertainties used in analyses by MiniBooNE were initially inflated by a factor of four to account for some inconsistencies within the production data. A more recent measurement by the SciBooNE collaboration indirectly measured the $K^{+}$production in the BNB to drastically reduce this uncertainty 16 .

With the outgoing particle production simulated, these particles are propagated with Geant4 taking into account energy loss and electromagnetic and hadronic processes, including the impact of the horn magnetic field on the kinematics of those particles. A custom decay model is used outside of the Geant4 framework to simulate the decay processes that result in neutrinos, which includes the latest branching fraction measurements and simulates polarization effects and kinematic distributions resulting from decay form factors. A number of techniques to enhance the statistical precision of the flux are employed [1].

The systematic uncertainties in the neutrino flux production come from several sources, which are summarized in Table 4.1. The dominant uncertainty is that from particle production. By varying parameters in all of these systematic sources, a systematic error envelope is calculated for the final neutrino flux through the MicroBooNE detector. Figure 4.4 shows the $\nu_{\mu}, \bar{\nu}_{\mu}, \nu_{e}$, and $\bar{\nu}_{e}$ flux at the MicroBooNE detector. The red bars show the systematic error envelope, which includes all errors except those for proton delivery, which is a flat normalization error. Table 4.2 summarizes the systematic errors for the integrated flux. 


\begin{tabular}{|l|l|}
\hline \multicolumn{2}{|c|}{ MicroBooNE BNB Flux Systematics } \\
\hline Source & Description \\
\hline \hline Proton delivery & $\begin{array}{l}\text { Counting the number of protons arriving on the beryllium } \\
\text { target. }\end{array}$ \\
\hline Particle production & $\begin{array}{l}\text { Rate and shape of secondary particles produced in p-Be in- } \\
\text { teractions. }\end{array}$ \\
\hline Hadronic interactions & The rate of hadronic interactions in target or horn. \\
\hline Horn magnetic field & $\begin{array}{l}\text { Magnetic field to focus the outgoing charged mesons from } \\
\text { p-Be interactions. }\end{array}$ \\
\hline Beam-line geometry & $\begin{array}{l}\text { Possible misalignments or displacements of beam-line com- } \\
\text { ponents from their expected orientations. }\end{array}$ \\
\hline Horn skin depth & Non-uniformity of current in the inner conductor of horn. \\
\hline \hline
\end{tabular}

Table 4.1: A summary of the systematic uncertainties included in the MicroBooNE flux prediction. The dominant uncertainty is that from particle production.

\begin{tabular}{|l|l|l|l|l|}
\hline & $\nu_{\mu}$ & $\bar{\nu}_{\mu}$ & $\nu_{e}$ & $\bar{\nu}_{e}$ \\
\hline POT Delivery & $2.00 \%$ & $2.00 \%$ & $2.00 \%$ & $2.00 \%$ \\
$\pi^{+}$ & $5.80 \%$ & $0.46 \%$ & $4.62 \%$ & $2.66 \%$ \\
$\pi^{-}$ & $0.01 \%$ & $7.51 \%$ & $0.28 \%$ & $3.20 \%$ \\
$K^{+}$ & $0.38 \%$ & $0.13 \%$ & $5.19 \%$ & $2.67 \%$ \\
$K^{-}$ & $0.01 \%$ & $0.35 \%$ & $0.28 \%$ & $3.92 \%$ \\
$K_{L}^{0}$ & $0.03 \%$ & $0.27 \%$ & $2.36 \%$ & $22.59 \%$ \\
Other & $5.78 \%$ & $6.09 \%$ & $3.60 \%$ & $7.61 \%$ \\
\hline Total & $8.44 \%$ & $9.89 \%$ & $8.43 \%$ & $24.74 \%$ \\
\hline \hline
\end{tabular}

Table 4.2: A summary of the systematic errors for the integrated flux, mostly from particle production. "Other" includes hadronic interactions, horn current uncertainty, and skin effect. 

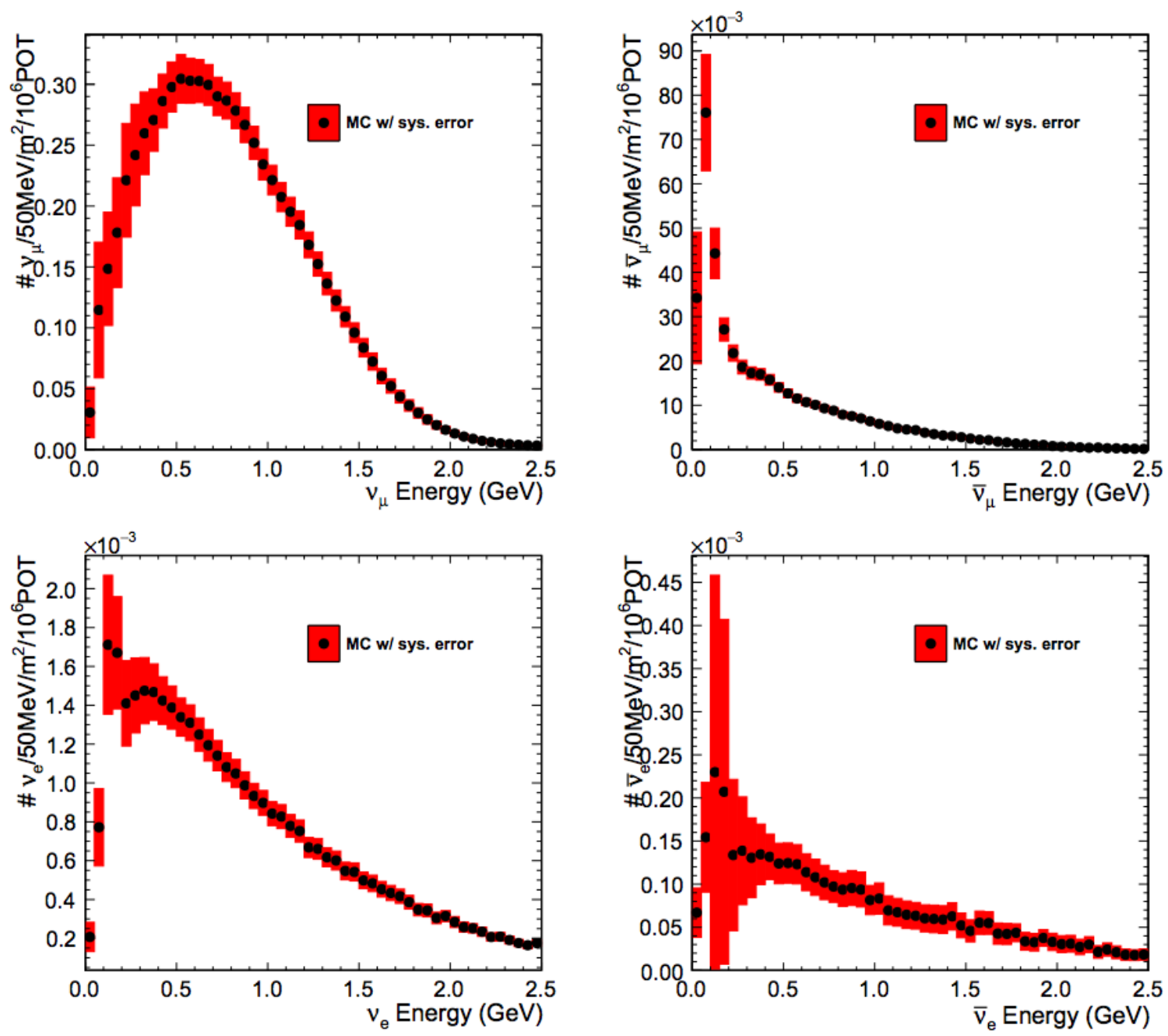

Figure 4.4: Neutrino flux prediction (black dots) with systematic error bars (red envelope) excluding proton delivery systematics which results in a flat normalization correction. 


\section{Chapter 5}

\section{Low Energy Excess: LSND and MiniBooNE}

\section{$5.1 \quad$ Introduction}

This chapter will describe the observed low energy excess reported by the MiniBooNE experiment and the electron antineutrino excess seen by the LSND experiment. The LSND experiment first observed an excess of $\bar{\nu}_{e}$ in a $\bar{\nu}_{\mu}$ beam in 2001. The MiniBooNE experiment then observed an unexplained excess of electron-like events in a primarily $\nu_{\mu}$ beam in the neutrino energy region between 200 to $475 \mathrm{MeV}$ in 2009. A description of the LSND observation followed by the MiniBooNE detector and analysis is provided in this chapter. The purpose of this chapter is to provide a historical motivation for the eventual sensitivity studies done for the MicroBooNE experiment, which will be described in Chapter 6 .

\subsection{LSND Observation}

In 2001, the Liquid Scintillator Neutrino Detector (LSND) collaboration published an observation of excess events consistent with $\bar{\nu}_{e}$ interactions above the expected background in a $\bar{\nu}_{\mu}$ beam at the Los Alamos Neutron Science Center 8. Given the $\frac{L}{E}\left(\approx \frac{30 m}{40 M e V}\right)$ for this measurement, the excess disagreed with previous neutrino oscillation measurements of 
the neutrino mixing angles and $\Delta m^{2}$ values in the three neutrino model. The LSND excess corresponded to a $\Delta \mathrm{m}^{2}$ of approximately $1 \mathrm{eV}^{2}$, orders of magnitude higher than previously measured values of $\Delta m_{12}^{2}$ and $\Delta m_{23}^{2}$. One explanation for this drastically different $\Delta m^{2}$ value is the possible existence of potential additional "sterile" neutrino states, which must not interact weakly given that the Z- boson decay width constrains the number of weakly interacting neutrino states to three.

To test the LSND result, the MiniBooNE experiment was designed. It would search for $\nu_{e}$ interactions in a primarily $\nu_{\mu}$ beam, with a similar $\frac{L}{E}\left(\approx \frac{500 \mathrm{~m}}{700 \mathrm{MeV}}\right)$.

\subsection{The MiniBooNE Experiment}

\subsubsection{The MiniBooNE Detector and Monte Carlo Simulation}

The MiniBooNE detector [17 consists of a spherical detector located 541 meters downstream of the BNB neutrino production target. As shown in Figure 5.1, the detector consists of a sphere with a diameter of 12.2 meters filled with 818 tons of mineral oil located underneath more than 3 meters of earth overburden. There exists a signal region instrumented with 1280 8-inch photomultiplier tubes (PMTs), most of which were reused from the LSND experiment, and a $35 \mathrm{~cm}$ thick outer veto region separated by an opaque barrier instrumented with 240 PMTs. The efficiency for rejecting cosmic ray muons by using the outer veto region was measured to be $99.99 \%$.

The detection method of the MiniBooNE experiment is based primarily on Cherenkov light. The mineral oil within the signal region acts as the neutrino target material. The majority of final state particles exiting neutrino interactions at neutrino energies from the BNB are produced above Cherenkov threshold. These particles produce Cherenkov light which is detected by the PMTs lining the signal region of the detector. Reconstructing the pattern of light projected onto the wall PMTs is used for particle identification.

The detector is calibrated with a series of in situ measurements, primarily with cosmic 

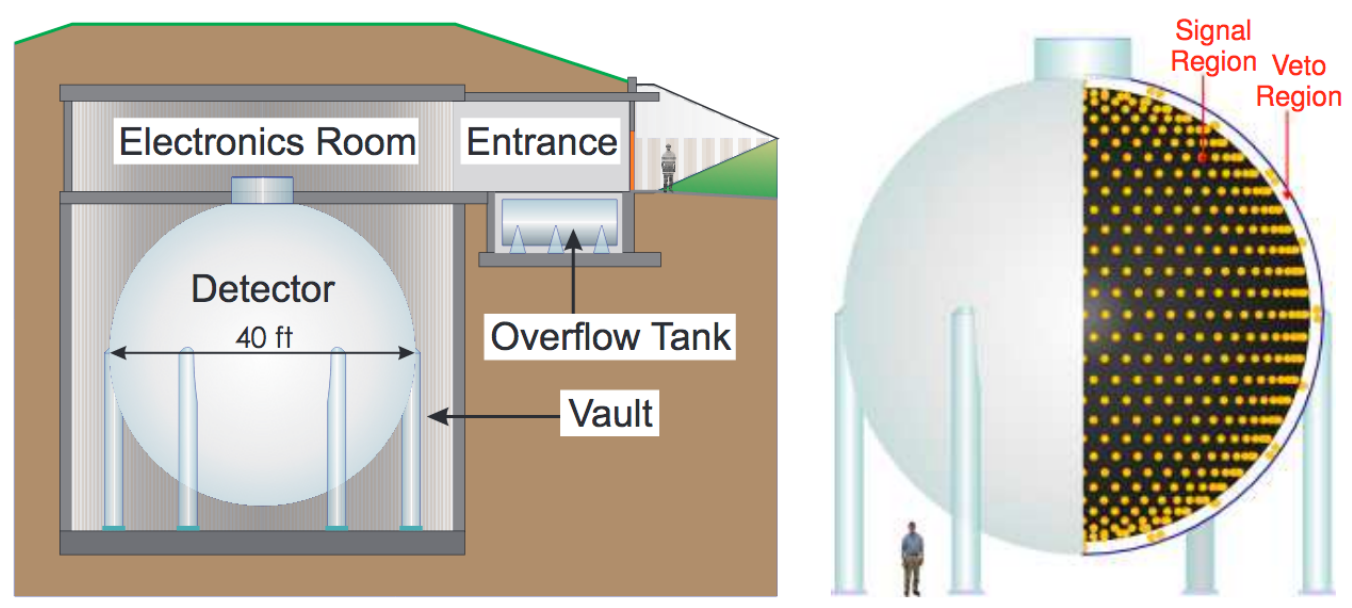

Figure 5.1: The MiniBooNE detector enclosure (left) and a cut-away drawing (right) of the detector showing the distribution of PMT's in the signal and veto regions.

ray muons. Cosmic ray muons stopping within the detector along with an external muon hodoscope provide for angular resolution measurements. Additionally, muons which stop and produce decay electrons that have a known energy endpoint of around $50 \mathrm{MeV}$ provide an energy calibration source at low energies, while through-going muons provide calibration information at higher energies. Also, tagged $\pi^{0}$ particles which decay into two photons have a known mass of around $135 \mathrm{MeV}$ and therefore provide energy calibration information in that region.

\subsubsection{MiniBooNE Event Selection}

Different final state particles exiting a neutrino interaction in the MiniBooNE signal volume create different patterns of Cherenkov light read out by the PMTs. Figure 5.2 18 shows how these patterns differ for different common kinds of final state particles (muons, electrons/photons, and neutral pion decays). A muon track produces a crisp, filled-in ring of Cherenkov light, while an electron or photon produces a more fuzzy, hollow ring. A neutral pion decay will result in two photons. By reconstructing these patterns in the PMT data read out from a triggered event in MiniBooNE, the flavor and energy of the interacting neutrino can be determined up to the energy lost to particles below Cherenkov threshold. 
With this kind of detection technique it is important to note that a single photon signal is indistinguishable from that of a single electron signal, a fact leading to the ultimate ambiguity of the observed low energy excess in MiniBooNE.

\section{particle track}

\section{Muon track}

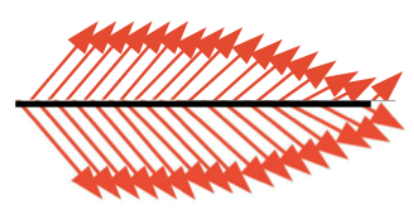

Electron, or photon

Neutral pion decay (2 photons)
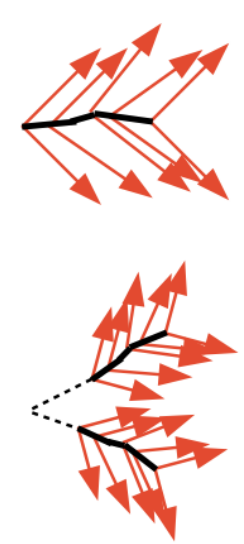

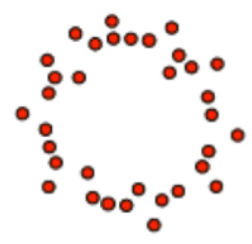

\section{Cherenkov ring}
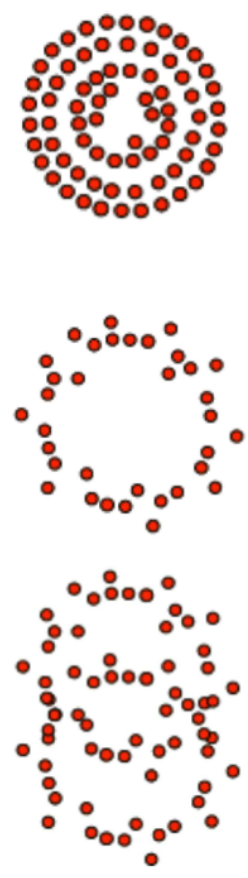

Figure 5.2: A schematic of the pattern Cherenkov light from different particles would make projected onto the inner walls of the MiniBooNE detector. Top is a muon track (a filled-in ring), middle is an electron (a fuzzy ring), bottom is a photon that pair-produces and creates two fuzzy rings.

The topology of interest in the MiniBooNE oscillation search is that of charged-current quasi-elastic (CCQE) interactions, shown in Figure 5.3. This interaction channel is the dominant one in the neutrino energy range of the BNB below $1 \mathrm{GeV} E_{\nu}$. In a $\nu_{l} \mathrm{CCQE}$ interaction (where $l$ is the neutrino flavor), a lepton of flavor $l$ is produced, along with a proton. The single outgoing lepton is the characteristic event signature for which Mini- 
BooNE searches since the proton is mostly below Cherenkov threshold.
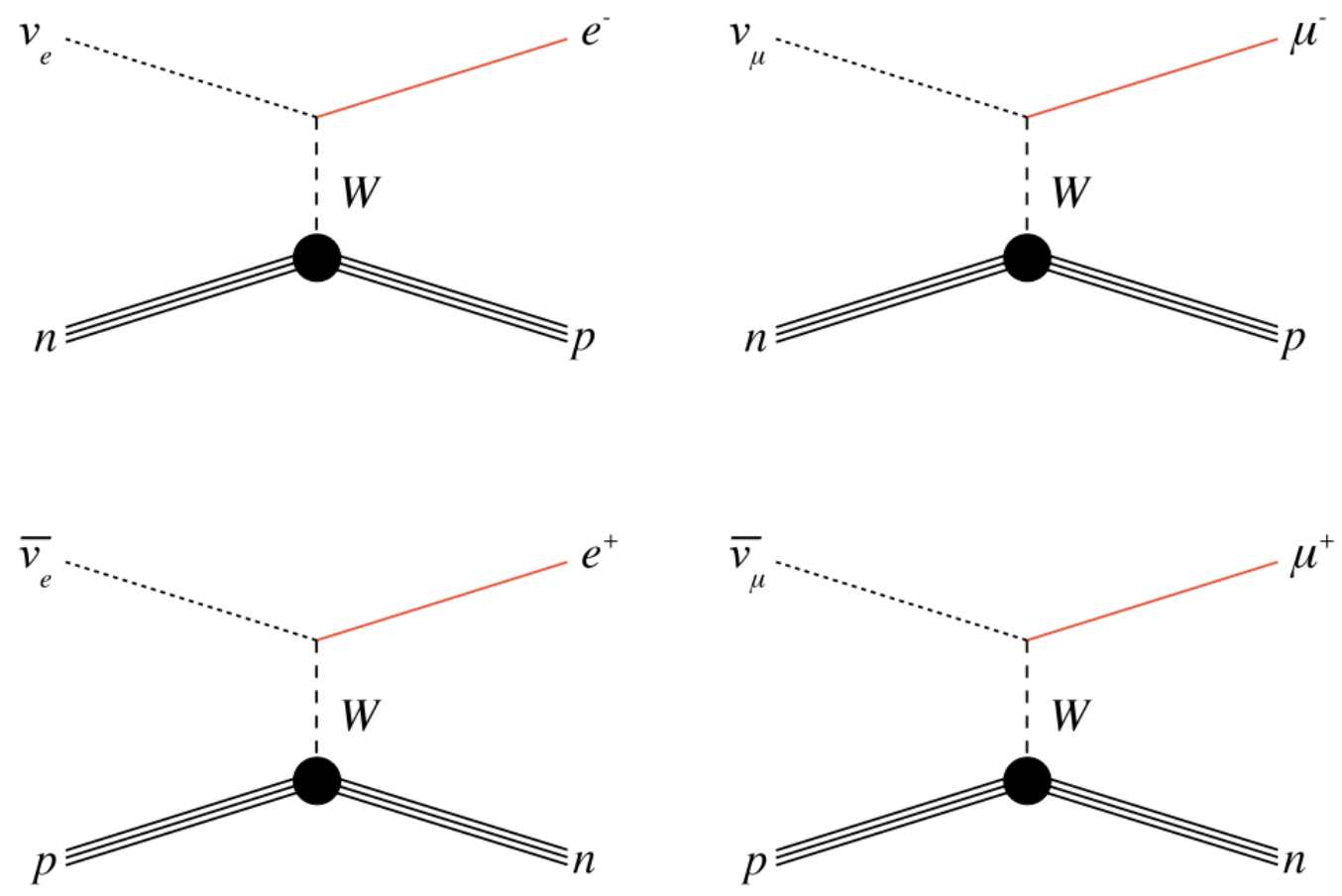

Figure 5.3: Feynman diagrams of the charged-current quasi-elastic (CCQE) interaction channel for $\nu_{e}, \nu_{\mu}, \bar{\nu}_{\mu}$, and $\bar{\nu}_{e}$ (clockwise from the top left). $\nu_{e}$ CCQE is the signal channel for the MiniBooNE oscillation analysis.

In order to select $\nu_{e}^{C C Q E}$ events, cuts are placed to mitigate backgrounds. The most powerful rejection comes from requiring the events occur within the beam timing window. The beam arrives at the detector at a rate of $5 \mathrm{~Hz}$, and each spill lasts $1.6 \mu s$ and is composed of approximately 80 buckets separated by 19 ns. Given the time scale with which MiniBooNE measures the light from interactions, cuts on event timing alone reduces nonbeam backgrounds to $\sim 10^{-3}$. Additional cuts require that there is significant activity in the signal volume and little activity in the outer veto volume, a signature characteristic of beam related neutrino events. These pre-cuts achieve more than a $99.99 \%$ rejection of beam unrelated backgrounds. The efficiency to select $\nu_{e}^{C C Q E}$ events in MiniBooNE depends on which cuts are applied in the analysis, but varies between $55 \%$ (with a purity of $36.8 \%$ ) and $26.6 \%$ (with a purity of $77.0 \%$ ) 19 . The efficiency to select $\nu_{e}^{C C Q E}$ events also depends on 
which cuts are applied but varies between $55.2 \%$ and $30.6 \% 20$.

In order to reconstruct events, MiniBooNE uses a maximum likelihood fitting algorithm leveraging properties of charged particle tracks and showers inferred from measured charges and times on the PMTs. The likelihoods associated with different event hypotheses are used to classify each event as a signal $\nu_{e} \mathrm{CCQE}$ event, or as a background process like $\nu_{\mu} \mathrm{CCQE}$ and NC $\pi^{0}$ production. Note that MiniBooNE cannot differentiate between a $\mu^{+}$and a $\mu^{-}$, or $e^{+}$and $e^{-}$so discrimination between neutrino and antineutrino on an event-by-event basis is not possible. MiniBooNE also uses the identified $\nu_{\mu}^{C C Q E}$ events to constrain the intrinsic $\nu_{e}$ background from muon decay and the measured $\mathrm{NC} \pi^{0}$, two-gamma rate to constrain the predicted single shower $\mathrm{NC} \pi^{0}$ backgrounds.

Assuming CCQE kinematics, the incident neutrino energy is reconstructed with knowledge of the outgoing lepton energy $\left(E_{l}\right)$ and scattering angle $\left(\theta_{l}\right)$. In MiniBooNE specifically, the struck nucleon is assumed to be at rest, so the incident neutrino energy $E_{\nu}^{C C Q E}$ is given by:

$$
E_{\nu}^{C C Q E}=\frac{2 m_{n} E_{l}+m_{p}^{2}-m_{n}^{2}-m_{l}^{2}}{2\left(m_{n}-E_{l}+\cos \theta_{l} \sqrt{E_{l}^{2}-m_{l}^{2}}\right)}
$$

where $m_{n}, m_{p}, m_{l}$ are the masses of the neutron, proton, and lepton respectively, and $\theta_{l}$ is the scattering angle of the outgoing lepton with respect to the (known) beam neutrino direction.

\subsubsection{MiniBooNE Results}

With the described reconstruction methods and energy definition, the MiniBooNE published results 21 for the $\nu_{e}$ appearance search in neutrino mode running are shown in Figure 5.4. Note that besides the irreducible intrinsic $\nu_{e}$ backgrounds, the dominant background in the excess region is $\pi^{0}$ mis-identification (MID) (red). In a $\pi^{0}$ MID event event, a $\pi^{0}$ is created in the neutrino interaction and its subsequent immediate decay into two photons can mimic the $\nu_{e}^{C C Q E}$ signature if either one of the photons escapes the detector or the Cherenkov 
rings from the two photons overlap to become one ring. Another important background is $\Delta \rightarrow N \gamma$ (brown). Recall that both of these backgrounds arise from MiniBooNE's inability to distinguish electrons from photons, an important ambiguity which will be discussed in more detail in the following sections.

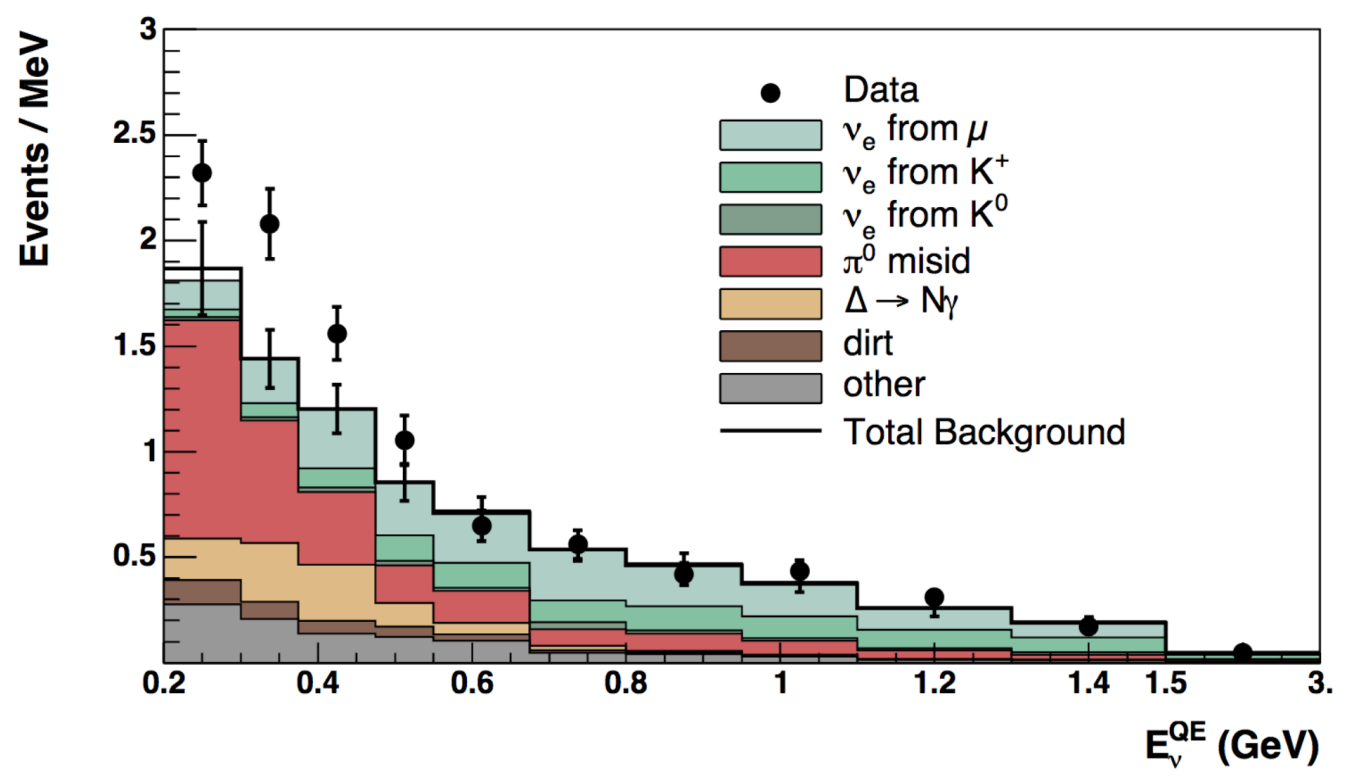

Figure 5.4: The $E_{\nu}^{Q E}$ distribution for MiniBooNE data (points with statistical errors) and and backgrounds (histogram with systematic errors).

As shown in Figure 5.4, in the energy region $E_{\nu}^{Q E}>475 \mathrm{MeV}$ there is good agreement between data and background prediction, making a two neutrino oscillation fit inconsistent with the LSND results at the $98 \%$ confidence level assuming CP conservation. Meanwhile, below $E_{\nu}^{C C Q E}$ of $475 \mathrm{MeV}$ there is a statistically significant $(6 \sigma$, reduced to $3 \sigma$ after systematics) excess. The excess of $129 \pm 43$ events (stat+syst) is consistent in magnitude with the LSND oscillation excess.

In a later separate antineutrino run (in which the BNB horn current is switched to produce a primarily $\bar{\nu}_{\mu}$ beam), an excess was observed in the energy region $E_{\nu}^{Q E}>475 \mathrm{MeV}$ that was consistent with an LSND-type two neutrino oscillation over the null oscillation 
hypothesis at the $91 \%$ confidence level. In the lower energy region $E_{\nu}^{Q E}<475 \mathrm{MeV}$, an excess of $38.6 \pm 18.5$ events was observed. In a fit to the full energy range $E_{\nu}^{Q E}>200$ $\mathrm{MeV}$, the excess was consistent with an LSND-type two neutrino oscillation over the null oscillation hypothesis at the $98 \%$ confidence level.

\subsubsection{Proposed Low Energy Excess Sources}

Shown in Figure 5.5 is the MiniBooNE neutrino mode excess (data - expected background) with oscillation fits with parameters constrained to be in the LSND allowed region. The parameters in the LSND allowed region are ruled out at the $95 \%$ confidence level if the data are fit with $E_{\nu}^{C C Q E}>475 \mathrm{MeV}$.

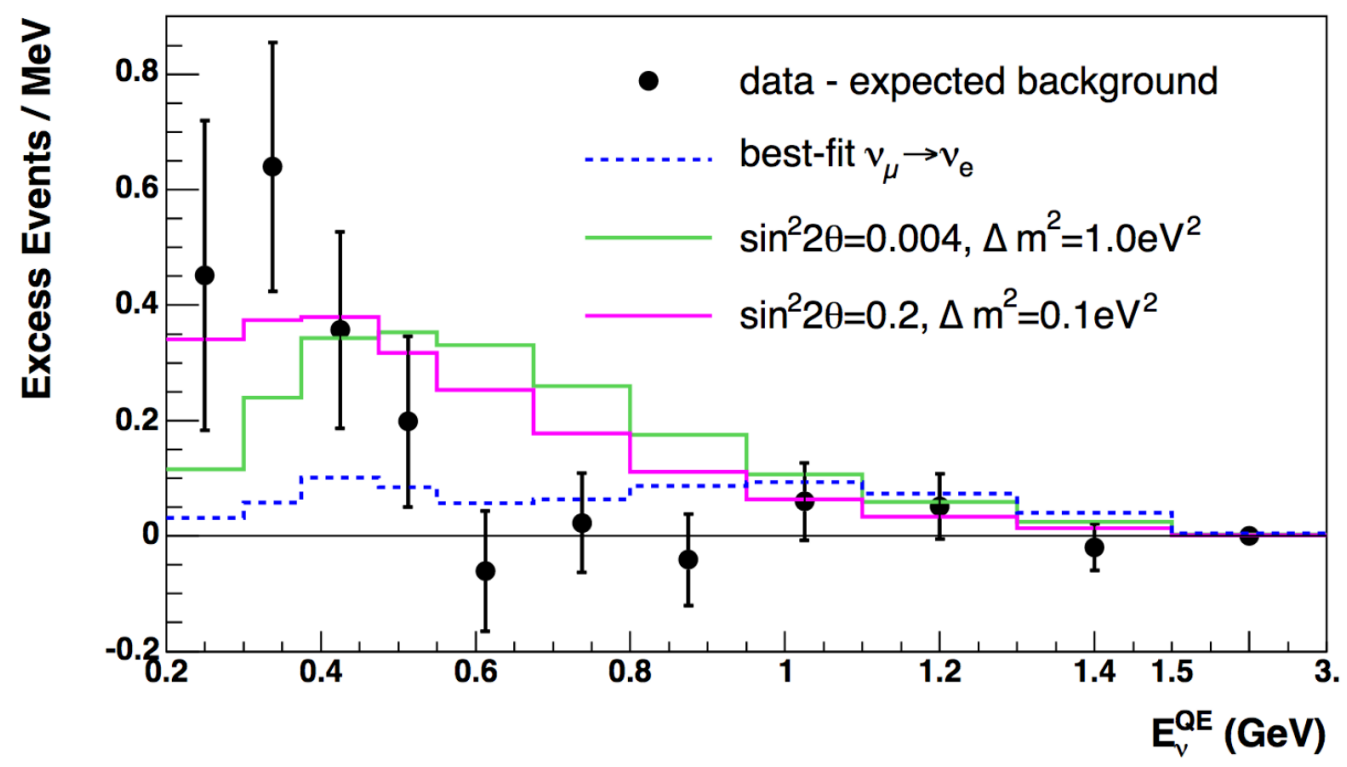

Figure 5.5: The MiniBooNE event excess as a function of $E_{\nu}^{Q E}$. Also shown are the expectations from the best oscillation fit and from neutrino oscillation parameters in the LSND allowed region. The error bars include both statistical and systematic errors.

Given MiniBooNE's inability to distinguish electrons from photons, the origin of this excess is either a mis-estimation of one of the backgrounds, or some sort of new physics. 
The former is unlikely the case because MiniBooNE makes many in situ measurements that allow for the constraining of these backgrounds. The neutral current induced backgrounds (NC $\pi^{0}, \Delta \rightarrow N \gamma$, and dirt) are constrained by such measurements. Measurements constraining these backgrounds are described in more detail in the following paragraphs.

The NC $\pi^{0}$ rate in MiniBooNE is measured by selecting events with reconstructed mass near the $\pi^{0}$ mass. This obtains a $>90 \%$ pure sample of $\mathrm{NC} \pi^{0}$ interactions which is compared to simulation to obtain a correction function in order to bring the simulated distribution in agreement with data. This same correction function is applied to NC $\pi^{0}$ events that are backgrounds in the $\nu_{e}$ appearance analysis. This correction function increases the $\mathrm{NC} \pi^{0}$ background by less than $13 \%$ for $E_{\nu}^{C C Q E}<400 \mathrm{MeV}$ and decreases the background by as much as $20 \%$ above this neutrino energy region. Including this correction factor, the uncertainty on the overall $\mathrm{NC} \pi^{0}$ backgrounds is $7 \%$. Note that a correction factor of 2.0 would be required to explain the origin of the excess as originating from a mis-estimated NC $\pi^{0}$ background 18 .

The excess is unlikely caused by a mis-estimation of the $\Delta \rightarrow N \gamma$ backgrounds because they are additionally constrained by the $\mathrm{NC} \pi^{0}$ measurement through the relative rate of resonant production times a branching fraction of $(0.56 \pm 0.04) \%$. With this measurement, the uncertainty on the $\Delta \rightarrow N \gamma$ backgrounds is $12 \%$. Note that a (very large) correction factor of 2.7 would be required to explain the origin of the excess as originating from a mis-estimated $\Delta \rightarrow N \gamma$ background.

The excess is unlikely caused by a mis-estimation of the dirt backgrounds because a direct measurement is made by selecting a separate event sample which are likely dirt events and comparing data to simulation. These events are reconstructed close to the detector boundaries with direction pointed generally inwards since this is a background from events produced outside of the detector. In neutrino mode, a dirt background normalization correction factor was computed to be $0.7 \pm 0.1$ (with simulation over-predicting the dirt rate normalization). Given the power of the event selection cuts designed to mitigate dirt back- 
grounds, the relevance of this relatively large correction factor is minimal.

The charged current induced backgrounds (intrinsic $\nu_{e}^{C C Q E}$ ) are reduced with in situ measurements of $\nu_{\mu} \mathrm{CCQE}$ interactions. A data to simulation comparison of measured $\nu_{\mu} \mathrm{CCQE}$ interactions allows for the retuning of underlying flux and cross section parameters in order to bring simulated distributions in agreement with data. These parameters are the same as those used to predict the $\nu_{e}^{C C Q E}$ rate and shape. In addition, a measurement of the highest energy $\nu_{\mu}$ CCQE interactions allows for the further constraint of $\nu_{e}^{C C Q E}$ from kaon decay backgrounds, which is discussed in more detail in a later section of this thesis.

Given the likelihood that the excess is not caused by misidentified backgrounds, several new-physics interpretations have been proposed in attempt to explain the excess, including sterile neutrino oscillations (with one, two, or more sterile neutrinos), and new interactions both within and outside of the standard model (CPT violation, quantum decoherence, sterile neutrino decay, etc). A summary of these interpretations can be found in 22. A commonality between all interpretations is that their interactions pass the MiniBooNE event selection cuts; that is, they have one electron or one photon exiting the interaction vertex.

\subsection{Conclusions}

This chapter has presented the historical background of the "low energy excess" observed by the MiniBooNE experiment published in 2009 that was in response to the oscillation signal reported by the LSND experiment in 2001. Possible sources for this excess have been discussed, such as mis-estimated backgrounds and sterile neutrinos. Ultimately the excess as seen by MiniBooNE can be attributed to an excess of either electron-like events or photon-like events. Determining which it is is beyond the capabilities of MiniBooNE due to limitations of its detector technology (a Cherenkov ring imaging detector). Because of this limitation, the MicroBooNE experiment was proposed in 2007 23. MicroBooNE was proposed to use the same neutrino beam in a similar location as MiniBooNE, but using 
a different detector technology with electron/photon separation powers (liquid argon time projection chambers) to search for and clarify the ambiguity in the low-energy excess. The detailed analysis aimed to quantify the sensitivity to a specifically electron-like MiniBooNE excess in the MicroBooNE detector is the subject of the next chapter of this thesis. 


\section{Chapter 6}

\section{Low Energy Excess: MicroBooNE}

This chapter will describe the MicroBooNE sensitivity study to observe the same excess as observed by MiniBooNE, in the same neutrino beam-line but with a different detector technology. The description of the analysis covers the signal modeling based on the MiniBooNE published data releases, the event selection, and background mitigation techniques employed. Ultimately, the expected sensitivity to measure such a signal is calculated.

\subsection{MicroBooNE In The Context of the Low Energy Excess}

Given that the proposed explanations for the origin of the measured MiniBooNE low energy excess in neutrino mode all predict either a single electron or single photon produced at the neutrino interaction vertex, and that MiniBooNE cannot discriminate between single electrons or photons, the MicroBooNE experiment was proposed in 2007. This detector (described in detail in Chapter 3) is a liquid argon time projection chamber, a relatively new detector technology which allows for the discrimination between single electrons and photons. MicroBooNE runs in the same beam line (BNB) in neutrino mode and is physically located close to MiniBooNE; the MicroBooNE detector is located $470 \mathrm{~m}$ from the BNB production target, while the center of the MiniBooNE detector is $541 \mathrm{~m}$ from the BNB production target. Therefore, MicroBooNE should be able to elucidate the MiniBooNE low energy excess ambiguity, first by seeing if an excess exists and then by determining if the excess is related to an excess of photon events or electron events. 
The electron/photon discrimination power of a LArTPC is based on the energy deposition at the start of electron and photon showers; photons will pair produce and in general have twice the ionization as a single electron. Shown in Figure 6.1 is the energy loss per unit length along the first $2.4 \mathrm{~cm}$ of simulated single electron showers (red) and single photon showers (black) in terms of minimally ionizing particle (MIP) energy in liquid argon (about $2.1 \mathrm{MeV} / \mathrm{cm}$ ). Leveraging the $\mathrm{dE} / \mathrm{dx}$ difference between electrons and photons, and additionally the presence of a several centimeter (on average) gap between a photon's creation point at the neutrino event vertex and its pair production, MicroBooNE has very powerful electron/photon separation power whereas MiniBooNE has none.

There are other important differences between the MiniBooNE detector and that of MicroBooNE which need to be considered when broaching the subject of estimating a sensitivity of a signal seen in MiniBooNE as it might be seen in MicroBooNE. These differences are summarized in Table 6.1. From the table it is clear that the event selection efficiency in MicroBooNE (not yet completely determined, but discussed in Section 6.4.3) will have to be much higher than that of MiniBooNE for the two experiments to have comparable statistical significances, since MicroBooNE is ten times smaller than MiniBooNE.

\subsubsection{Past Sensitivity Studies}

The initial attempt to scale the MiniBooNE backgrounds and excess to MicroBooNE is shown in Figure 6.2, both under the assumption that the excess is due to an electron-like event (left) or due to a photon-like event (right) 24. Performing such a scaling analysis is a subtle and difficult task because of the drastic differences between the MiniBooNE and MicroBooNE detectors, as described in the previous section.

This scaling assumes the electron/photon misidentification rate in MicroBooNE is $6 \%$ (whereas it is $100 \%$ for MiniBooNE). Also, event selection efficiencies in MicroBooNE are assumed to be exactly twice that of MiniBooNE because of the detector technology. This scaling procedure ignores other potentially important differences between MicroBooNE and 


\begin{tabular}{|l|l|l|}
\hline \multicolumn{2}{|c|}{ MiniBooNE Compared to MicroBooNE } \\
\hline & MiniBooNE & MicroBooNE \\
\hline \hline Detector Technology & Cherenkov & LArTPC \\
\hline Nominal POT & $6.46 \times 10^{20}$ & $6.6 \times 10^{20}$ \\
\hline Active Volume Mass & 818 Tons & 89 Tons \\
\hline Signal Selection Efficiency & $25 \%$ & N/A \\
\hline Readout Time Scale & nanoseconds & milliseconds \\
\hline $\begin{array}{l}\text { Distance from Neutrino Pro- } \\
\text { duction Target }\end{array}$ & $541 \mathrm{~m}$ & $470 \mathrm{~m}$ \\
\hline Target Material & & \\
\hline e $\rightarrow \gamma$ Separation Power & None & Argon \\
\hline Sensitive to Vertex Activity & No & High \\
\hline \hline
\end{tabular}

Table 6.1: A comparison of some of the important similarities and differences between the MiniBooNE detector and the MicroBooNE detector, which make the signal modeling in this sensitivity study difficult. 
Energy loss In the flrst $24 \mathrm{~mm}$ of track: $250 \mathrm{MeV}$ electrons vs. $250 \mathrm{MeV}$ gammas

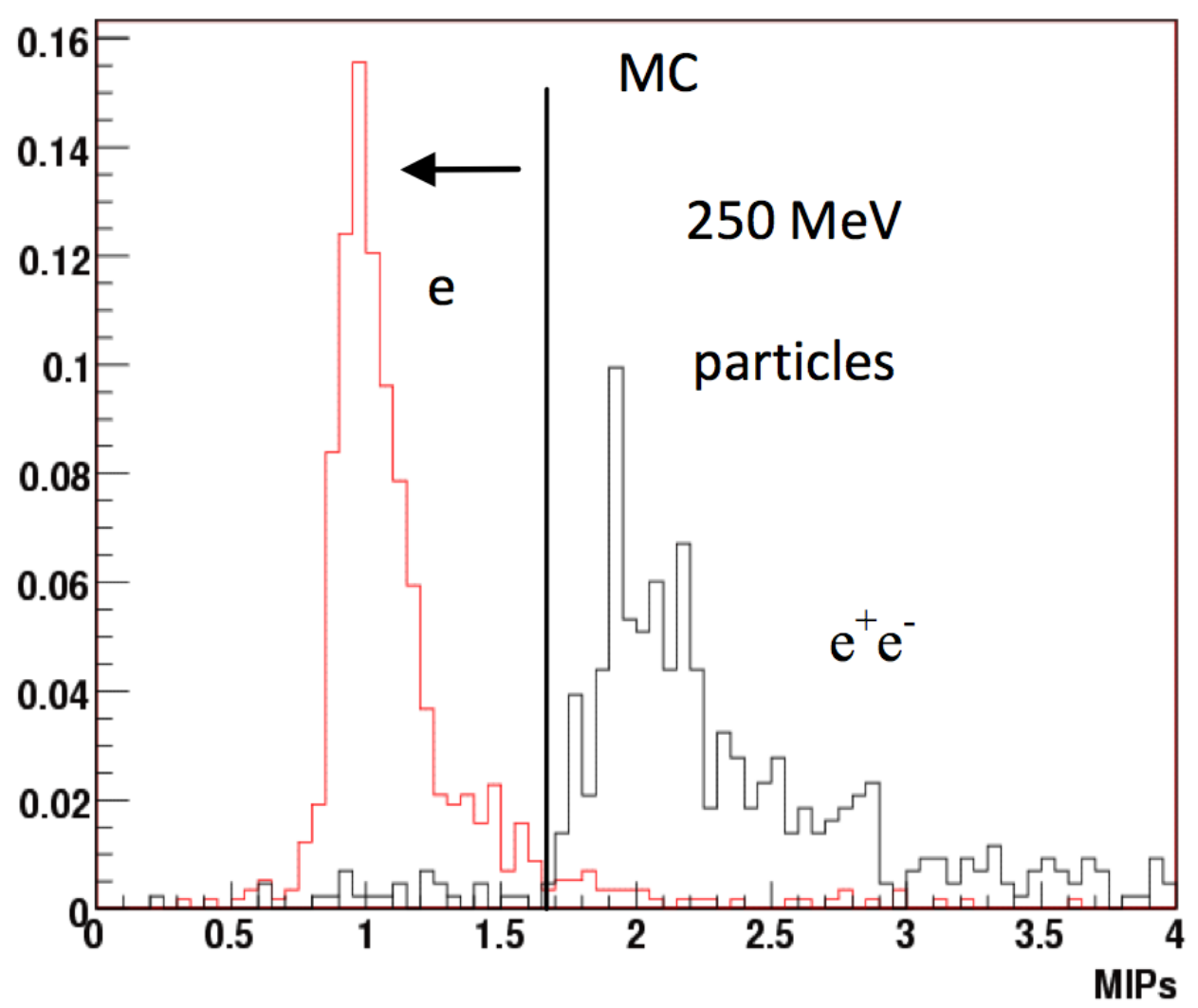

Figure 6.1: Energy loss per unit length along the first $2.4 \mathrm{~cm}$ of simulated single electron showers (red) and single photon showers (black) in terms of minimally ionizing particle (MIP) energy in liquid argon (about $2.1 \mathrm{MeV} / \mathrm{cm}$ ). Photons in general have twice the $d E / d x$ of electrons at the start of their showers, stemming from pair production.

MiniBooNE including differences in detector geometry (important for $\pi^{0}$ mis-identifications in which one photon escapes), flux differences (the relative rates are roughly $25 \%$ different because of the baseline differences of the two detectors), event topology selection differences (MicroBooNE can see much more vertex activity than can MiniBooNE, especially when additional final state particles are below Cherenkov threshold), the differing cosmic rejection background efficiencies (MiniBooNE can reject cosmics more efficiently than MicroBooNE because of their outer veto and because of the differing detection timescales 

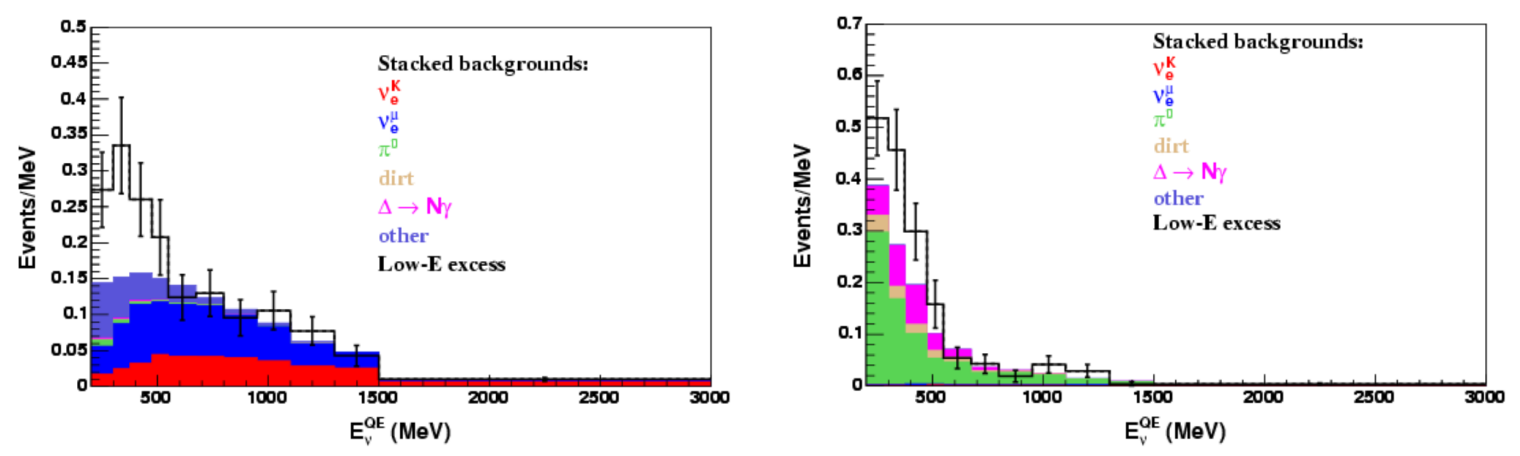

Figure 6.2: The results of the first analysis to scale the MiniBooNE backgrounds and excess to MicroBooNE both under the assumption that the excess is due to an electron-like event (left) or under a photon-like event (right). Stacked histograms show the expected background. Error bars indicate statistical uncertainty. The number of signal events, scaled from MiniBooNE for neutrino flux and fiducial volume, is the same in both plots (though dedicate electron-specific and photon-specific event selection cuts may show this to be unrealistic). Both plots assume $6.6 \times 10^{20}$ POT for the MicroBooNE 60 ton fiducial mass.

of Cherenkov technology, $n s$, compared to that of LArTPCs, $m s$ ), cross section differences between argon and $\mathrm{CH}_{2}$ arising from differing proton to neutron ratios, among other things.

The resulting statistical significance from the aforementioned scaling (previous Ref. 24]) after the nominal amount of data is taken in MicroBooNE $\left(6.6 \times 10^{20} \mathrm{POT}\right)$ is computed to be $5.7 \sigma$ under the single-electron excess hypothesis and $4.1 \sigma$ under the single-photon hypothesis.

The described scaling analysis is a very valuable tool, but is fraught with many questionable assumptions. The next sections in this thesis describe a more rigorous analysis with the ultimate goal of computing MicroBooNE's sensitivity to the MiniBooNE low energy excess assuming specifically the single-electron hypothesis. In this analysis, actual signal and background events will be simulated in the MicroBooNE detector and event selection cuts and algorithms will be used to select them. 


\subsection{Monte Carlo Simulation}

\subsubsection{Simulated Background Samples}

In this thesis analysis, both beam induced backgrounds and beam external backgrounds are simulated in the MicroBooNE cryostat. For beam-induced samples, the same flux predictions are used as were used in the MiniBooNE simulations (accounting for baseline and acceptance differences). The beam-induced samples come from full simulated BNB interactions with cross sections provided by GENIE 25[1. Non-beam samples (cosmics) come from simulated CORSIKA generated [27] cosmic rays that pass through the cryostat. Cosmic rays passing through other portions of the detector hall but not the cryostat result in negligible backgrounds in this $\nu_{e}$ search. The passage of all particles through the detector volume is simulated by the GEANT4 package 12 .

\subsubsection{Reconstruction}

In general, the output of an automated reconstruction chain in a LArTPC consists of reconstructed optical hits which come from the PMT signals, and reconstructed wire hits which come from drift electron ionization signals on the induction and collection plane wires. Reconstruction algorithms cluster the electron ionization hits on each wire plane into those corresponding to individual particles, then match clusters from different wire planes to form $3 \mathrm{D}$ reconstructed objects. The wire planes provide two of the three dimensions, and matching clusters to the timing of optical hits on the PMTs provide the third (drift) dimension. These reconstructed objects are either thin, straight tracks (which come from particles like muons, charged pions, and protons) or more fuzzy cone-shaped showers, which come from higher energy electrons or photons. Ideally this analysis would be done using these reconstructed objects. In this way, the same event selection methods could be used on data as are used in simulation. While automatic track reconstruction can currently be performed

\footnotetext{
${ }^{1}$ Note that while MicroBooNE uses GENIE to simulate BNB interactions, MiniBooNE used NUANCE 26. Given the approach to determine the absolute normalization of the MiniBooNE excess as seen in MicroBooNE based on intrinsic $\nu_{e}$ rates (see Section 6.5, differences between these two generators can be ignored.
} 
at an adequate level, the difficulties involved in shower reconstruction (which is particularly important to tag and study $\nu_{e}^{C C}$ events) have yet to be overcome by MicroBooNE and the LArTPC community. Currently, the MicroBooNE collaboration is moving to use the methods and algorithms of the analysis presented here to do a sensitivity estimate using reconstructed objects.

For these reasons, this simulation-only study is done with objects that are not automatically reconstructed from wire and PMT signals, but instead from truth-based energy depositions in the detector. In general, these objects represent what would be reconstructed from wire and PMT signals if the reconstruction algorithms performed perfectly. Therefore, this is referred to as "perfect reconstruction" and the details of these objects are discussed in the next section.

\subsubsection{1 "Perfect Reconstruction"}

While a simulation-only study using real automated reconstruction would be ideal, such a study using "perfect reconstruction" is incredibly valuable as an initial estimate of the MicroBooNE sensitivity; it is a step forward from the aforementioned scaling study (Section 6.1.1), and the event selection cuts and algorithms designed in this study can be used out-ofthe-box on automated reconstructed objects once they become available. Additionally, the "perfect reconstruction" can be tuned to more realistically represent what automated reconstruction might be capable of, for example by smearing the energy of objects or emulating realistic reconstruction efficiencies. This provides the important estimate of uncertainties arising from the imperfect automated reconstruction.

As mentioned earlier, the final 3D reconstructed objects formed from wire plane signals and PMT signals are referred to as tracks or showers. Tracks are close to straight lines in three dimensions, while showers are fuzzier and generally cone-shaped in three dimensions. The "perfect reconstruction" analogs to tracks and showers are referred to as MCTRACKS and MCShowers. They are created from simulated GEANT4 3D energy depositions in the detector volume. GEANT4 outputs 3D energy depositions in the detector, along with 
truth information about which parent particles deposited this energy. MCSHOwers and MCTRACKS are 3D objects which are formed by grouping energy depositions based on parent particles. Whether a particle in GEANT4 becomes an MCSHOwER or an MCTRACK is based on truth particle identity. For example, electrons always form MCSHOwer $2^{2}$ and muons always form MCTRACKs. All of the energy deposited by particles within the TPC is used to form these "perfect reconstructed" objects, which is in line with them representing actual reconstructible quantities (no ionization outside of the TPC is reconstructible).

MCTRACKS consist of a series of ordered 3D trajectory points, each corresponding to an energy deposition in the detector. MCSHOwERs have the following attributes: 3D start point where the first energy from the parent particle is deposited, 3D direction which is computed by fitting a line in 3D to all of the deposited energy from the parent particle, and $\mathrm{dE} / \mathrm{dx}$ computed from the energy depositions along the first few centimeters of the shower. These "perfectly reconstructed" tracks and showers (MCTRACKS and MCSHOwERS) serve as the input to the event selection algorithms, just as automated reconstructed tracks and showers (with the same attributes) would in real data.

\subsection{Event Selection}

This section describes the algorithms and cuts used to identify $\nu_{e}^{C C}$ interactions, given as input the "perfect reconstructed" MCTRACKS and MCSHOwERs from simulated triggered events in MicroBooNE ${ }^{3}$. Note that while the initial event selection algorithms are designed to identify $\nu_{e}^{C C}$ inclusive interactions (which may involve pions in the final state, for example), ultimately only the $\nu_{e}^{C C Q E}$ events (with only one electron and protons in the final state) are used in the final sensitivity estimates.

\footnotetext{
${ }^{2}$ Despite the fact that electrons behave more like tracks below the critical energy, which is on the order of $40 \mathrm{MeV}$ in liquid argon.

${ }^{3}$ Note that these cuts and algorithms could use automatically reconstructed tracks and showers, and therefore could be run both on simulation and data, if the quality of track and shower reconstruction was high enough.
} 
To select $\nu_{e}^{C C}$ interactions in MicroBooNE, a series of nine algorithms are run, each with a specific goal in mind; they either identify background topologies in order to remove them, or they identify the signal $\nu_{e}^{C C}$ topology. For example, one algorithm identifies MCSHowERS which are likely delta rays originating from tracks. Once identified, these MCSHOwERS are no longer candidate $\nu_{e}^{C C Q E}$ electrons. Another algorithm identifies pairs of showers that are likely from $\pi^{0}$ decays (by using the $\mathrm{dE} / \mathrm{dx}$ of those showers to identify them as photons, and requiring they back-project to a common origin) in order to remove them from the pool of candidate $\nu_{e}^{C C}$ electrons. Another algorithm tags through-going tracks as cosmic in origin, ensuring they will not be associated with a beam neutrino interaction.

The two most important event selection algorithms for this analysis are named "AlgoEMPart" (which handles the electron/photon discrimination) and "AlgoSingleE" (which is the algorithm responsible for locating the $\nu_{e}^{C C}$ topology and associating all tracks and showers together for eventual energy reconstruction and analysis). These two algorithms are discussed in detail in the following two subsections. At the end of the chain of event selection algorithms, a sample of candidate $\nu_{e}^{C C}$ events is obtained. These events are the subject to further cuts both to mitigate some backgrounds that the event selection algorithms missed,

and more importantly to pick specifically $\nu_{e}^{C C Q E}$ events. This down-sampling is necessary to make the eventual comparison to the MiniBooNE excess, since MiniBooNE searched exclusively for $\nu_{e}^{C C Q E}$ events, not $\nu_{e}^{C C}$ inclusive.

\subsubsection{Electron/Photon Separation Algorithm}

Electron/photon separation based on $\mathrm{dE} / \mathrm{dx}$ at the start of showers is done through an algorithm called "AlgoEMPart". This algorithm uses trained likelihood distributions which input $\mathrm{dE} / \mathrm{dx}$ and return the likelihood that the shower is electron-like, or photon-like. If a conversion distance (the distance between the reconstructed neutrino event vertex and the first energy deposition of the shower) is known, it will incorporate that into its likelihood as well. This additional handle is powerful because in general an electron shower will have a near-zero conversion distance, while a photon shower will often have a conversion distance 
of several centimeters. The algorithm's likelihood is configured with parameters output by a RooFit 28 minimization routine. The RooFit routine is trained on simulated single electron and single photon MCSHOwERs. In general, this algorithm computes both the likelihood that an MCSHOwER is an electron and that it is a photon, and determines the identity of the particle to be the one with the larger likelihood.

There are two likelihood functions that may be used. If a shower can independently be associated with a neutrino interaction vertex, the algorithm will use a $2 \mathrm{D}$ likelihood function that includes both $\mathrm{dE} / \mathrm{dx}$ and conversion length information. If an algorithm cannot associate a vertex with a shower, there is a 1D likelihood function that can be used with only $\mathrm{dE} / \mathrm{dx}$ information. The $1 \mathrm{D}$ likelihood function is composed of a Gaussian plus a landau distribution for $\mathrm{dE} / \mathrm{dx}$, which are combined to better parameterize the $\mathrm{dE} / \mathrm{dx}$ distribution, and the 2D likelihood function also includes an exponential function for conversion length. Any potential energy dependence on $\mathrm{dE} / \mathrm{dx}$ or conversion distance is not included in these likelihoods. The twelve trained input parameters include mean and sigma values for the Gaussian distributions, the most probable value (MPV) and sigma values for the landau distributions, the fractional area difference between the Gaussian and landau distributions ("frac"), and the conversion length parameter (six parameters for electrons, six parameters for photons). When training, input parameters for each sample (electron, photon) are the MCSHOwer computed $\mathrm{dE} / \mathrm{dx}$ as well as the truth-level creation vertex of the particle. The training results on "perfect reconstruction" electron and photon showers are shown in Figure 6.3 .

\subsubsection{Performance}

The performance of this algorithm on "perfect reconstruction" is computed by using samples of single electron showers and single photon showers generated isotropically between 0.05 and $2 \mathrm{GeV}$, and selecting those events where greater than $90 \%$ of the shower's energy is contained within the TPC. The algorithm's likelihood is trained using this sample (integrated over the full energy range of the showers). The efficiency to tag electrons and

photons both with the $1 \mathrm{D}$ and the $2 \mathrm{D}$ likelihood are enumerated below. Note that there are 


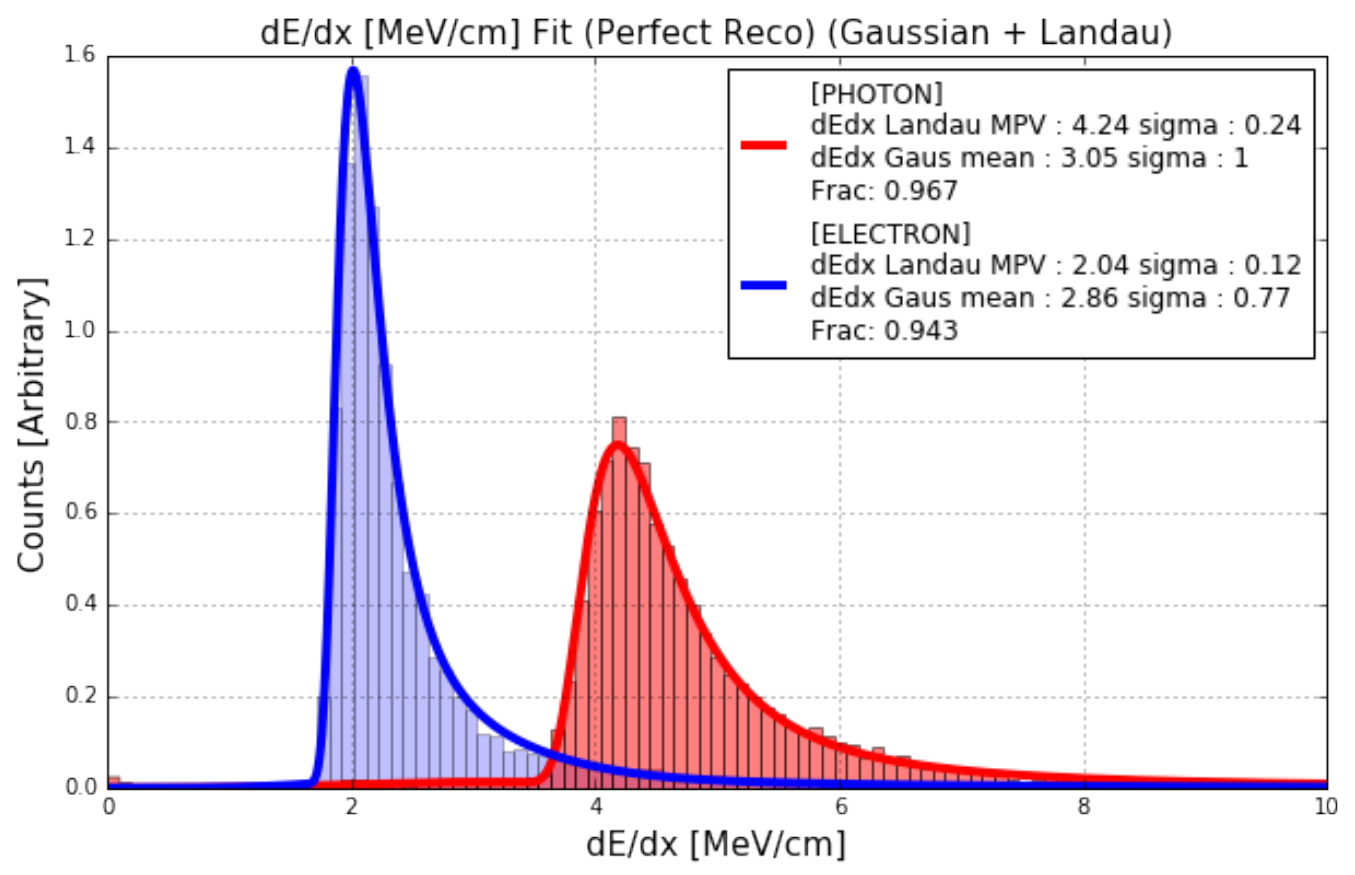

Figure 6.3: AlgoEMPart training results on perfect reconstructed electron showers and on perfect reconstructed photon showers as described in Section 6.3.1.1: 1D landau + Gaussian fit to $d E / d x$. "Frac" in the legend is the relative normalization between the landau and Gaussian fits; that "Frac" is close to one means the landau fit dominates.

additional handles to identify photons which are part of a $\pi^{0}$ decay which are not included in the efficiencies below (which are for single electrons or single photons only).

1. Using only $d E / d x$ information, the efficiency (over all energies) to select a single electron is $93 \%$, while the MID efficiency to tag the electron as a photon is $7 \%$.

2. Using only $d E / d x$ information, the efficiency to select a single photon is $97.3 \%$, while the MID efficiency to tag the photon as an electron is $2.7 \%$.

3. Using both $d E / d x$ and radiation length information (using the true creation point of photons), the efficiency to select a single electron is $99.7 \%$, while the MID efficiency to tag the electron as a photon is $0.3 \%$.

4. Using both $d E / d x$ and radiation length information (using the true creation point of photons), the efficiency to select a single photon is $98.1 \%$, while the MID efficiency to 
tag the photon as an electron is $1.9 \%$.

The 1D likelihood to determine if a shower is electron-like or photon-like is shown in Figure 6.4. The likelihood that a shower with a given $\mathrm{dE} / \mathrm{dx}$ is electron-like is computed by the ratio of the $1 \mathrm{D}$ electron-like probability distribution function $(\mathrm{PDF})$ value for that $\mathrm{dE} / \mathrm{dx}$ (shown non-normalized in Figure 6.3) to the sum of the electron-like PDF value for that $\mathrm{dE} / \mathrm{dx}$ and the photon-like PDF value for that $\mathrm{dE} / \mathrm{dx}$ (shown non-normalized in Figure 6.3 as well),

$$
L_{e}=\frac{e_{d E / d x}^{P D F}\left(\frac{d E}{d x}\right)}{e_{d E / d x}^{P D F}\left(\frac{d E}{d x}\right)+g_{d E / d x}^{P D F}\left(\frac{d E}{d x}\right)}
$$

where $e_{d E / d x}^{P D F}\left(\frac{d E}{d x}\right)$ represents the electron $\mathrm{dE} / \mathrm{dx}$ PDF function (shown non-normalized in Figure 6.3 evaluated at a $\mathrm{dE} / \mathrm{dx}$ value $\frac{d E}{d x}$ and $g_{d E / d x}^{P D F}\left(\frac{d E}{d x}\right)$ represents the photon $\mathrm{dE} / \mathrm{dx}$ PDF function (also shown non-normalized in Figure 6.3 evaluated at a $\mathrm{dE} / \mathrm{dx}$ value $\frac{d E}{d x}$. The likelihood that a shower with a given $\mathrm{dE} / \mathrm{dx}$ is photon-like is similarly computed but with the photon-like PDF value for that $\mathrm{dE} / \mathrm{dx}$ in the numerator.

The $2 \mathrm{D}$ likelihood including both $\mathrm{dE} / \mathrm{dx}$ and conversion distance is shown in Figure 6.5. The likelihood that a shower with a given $\mathrm{dE} / \mathrm{dx}$ value, $\frac{d E}{d x}$ and a given conversion distance value, $d$ is electron-like is computed as follows:

$$
L_{e}=\frac{e_{d E / d x}^{P D F}\left(\frac{d E}{d x}\right) * e_{\text {conv }}^{P D F}(d)}{g_{d E / d x}^{P D F}\left(\frac{d E}{d x}\right) * g_{\text {conv }}^{P D F}(d)}
$$

where $e_{d E / d x}^{P D F}\left(\frac{d E}{d x}\right)$ represents the electron $\mathrm{dE} / \mathrm{dx}$ PDF function (shown non-normalized in Figure 6.3 evaluated at a $\mathrm{dE} / \mathrm{dx}$ value, $\frac{d E}{d x}, e_{\text {conv }}^{P D F}(d)$ represents the electron conversion distance PDF function (shown non-normalized in Figure 6.6, noting that the electron conversion distance is effectively zero so the exponential fits the distribution relatively poorly) evaluated at a conversion distance value, $d, g_{d E / d x}^{P D F}\left(\frac{d E}{d x}\right)$ represents the photon $\mathrm{dE} / \mathrm{dx}$ PDF function (shown non-normalized in Figure 6.3 evaluated at a $\mathrm{dE} / \mathrm{dx}$ value, $\frac{d E}{d x}, g_{\operatorname{conv}}^{P D F}(d)$ represents the photon conversion distance PDF function (shown non-normalized in Figure 6.7) evaluated at a conversion distance value, $d$. The likelihood that the same shower is 
photon-like is simply the inverse of Equation 6.2.

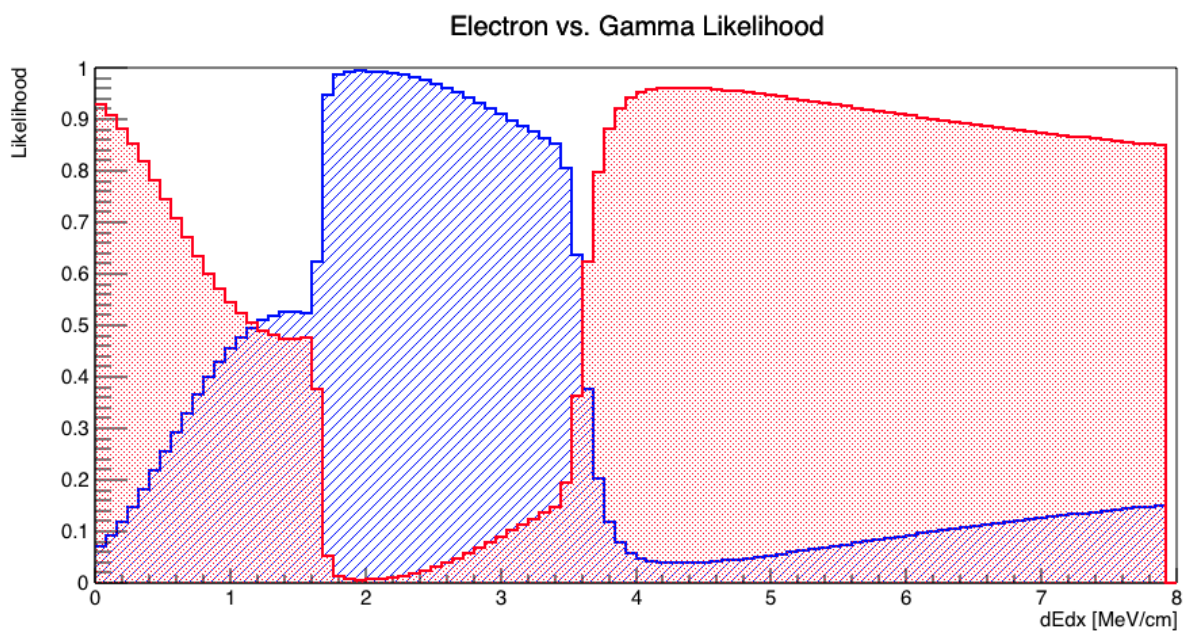

Figure 6.4: AlgoEMPart: Computed 1D likelihood vs $d E / d x$ : red is photon, blue is electron. How the likelihood is computed is described in Section 6.3.1.1.

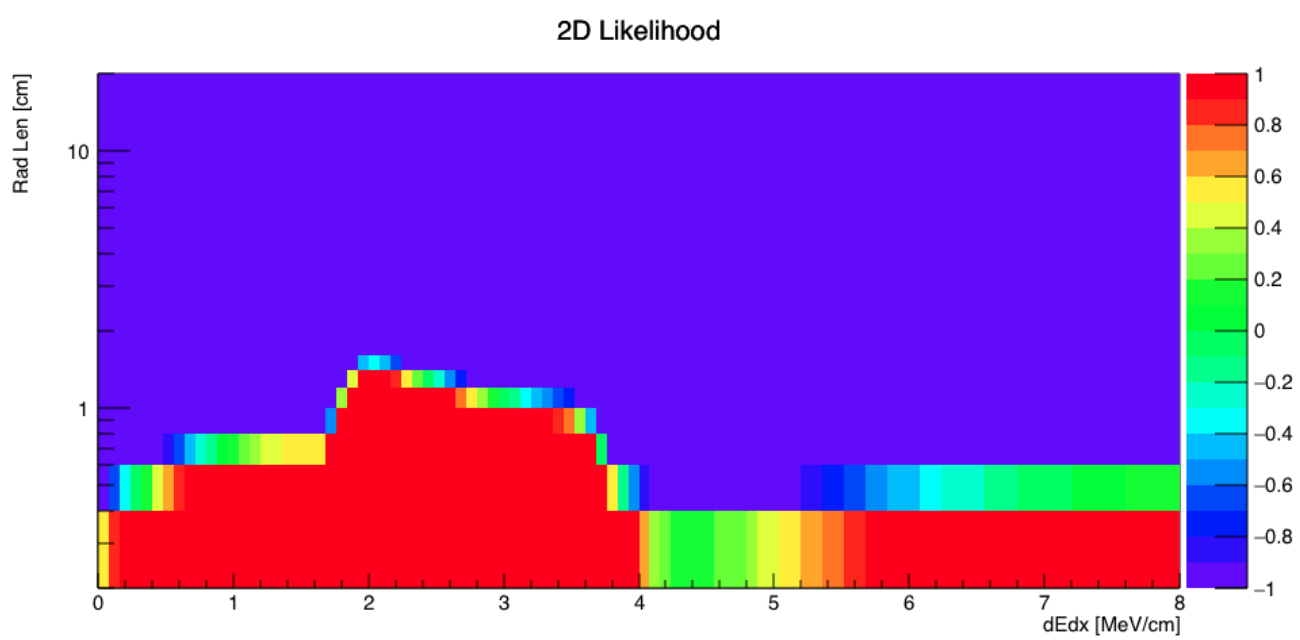

Figure 6.5: AlgoEMPart training results on perfect reconstructed electron and photon showers as described in Section 6.3.1.1 integrated over all energies: 2D likelihood distribution (radiation length vs. $d E / d x$ ). Low values of likelihood (purple) correspond to photon-like, high values (red) correspond to electron-like. 


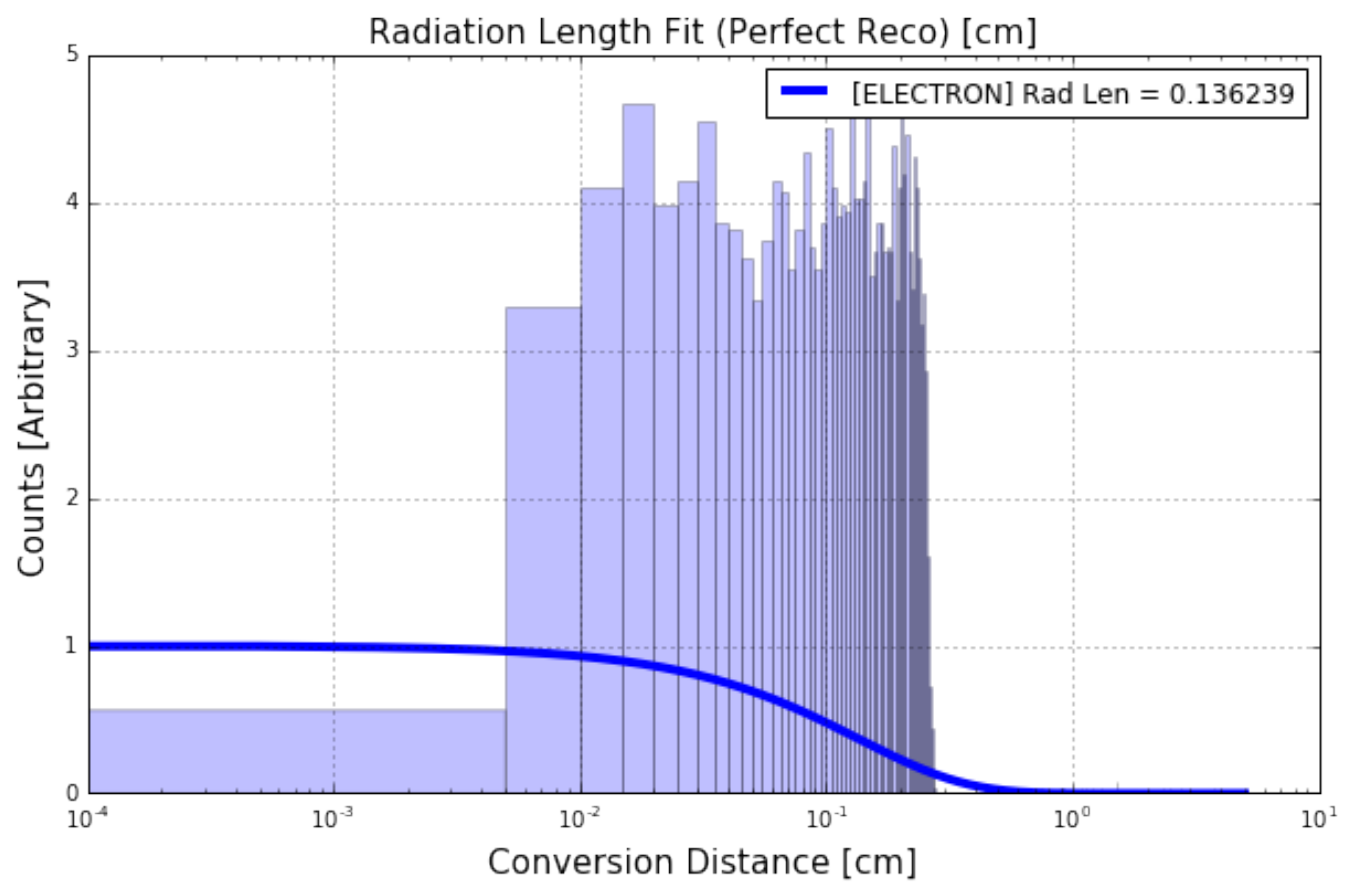

Figure 6.6: AlgoEMPart training results on perfect reconstructed electron showers as described in Section 6.3.1.1: Radiation length fit to single electron showers. Note the poor quality of the fit as the electron conversion distance for "perfect reconstruction" does not follow an exponential distribution; all conversion distances are below 0.3 centimeters.

\subsubsection{Signal Selection Algorithm}

The purpose of this algorithm is to select events with $\nu_{e}^{C C}$ inclusive type topologies. These topologies involve a single electron at a neutrino interaction vertex, with any number of protons, charged or neutral pions, or anything else additionally exiting the vertex. Later on, the sample of selected events will be subjected to further cuts to select only $\nu_{e}^{C C Q E}$ topologies by rejecting events with pions in the final state. This algorithm uses likelihoods provided by the previously described "AlgoEMPart" (Section 6.3.1) to determine if a shower is an electron or a photon. This algorithm begins by looping over all candidate $\nu_{e}^{C C}$ electron showers in the event that have not been removed by upstream cosmic and $\pi^{0}$ tagging algorithms. Figure 6.9 is a flowchart depicting the decision tree this algorithm uses for each candidate $\nu_{e}^{C C}$ electron shower. If the algorithm reaches the bottom of the flowchart, that shower is determined to be from a $\nu_{e}^{C C}$ interaction and the event is saved to be included in 


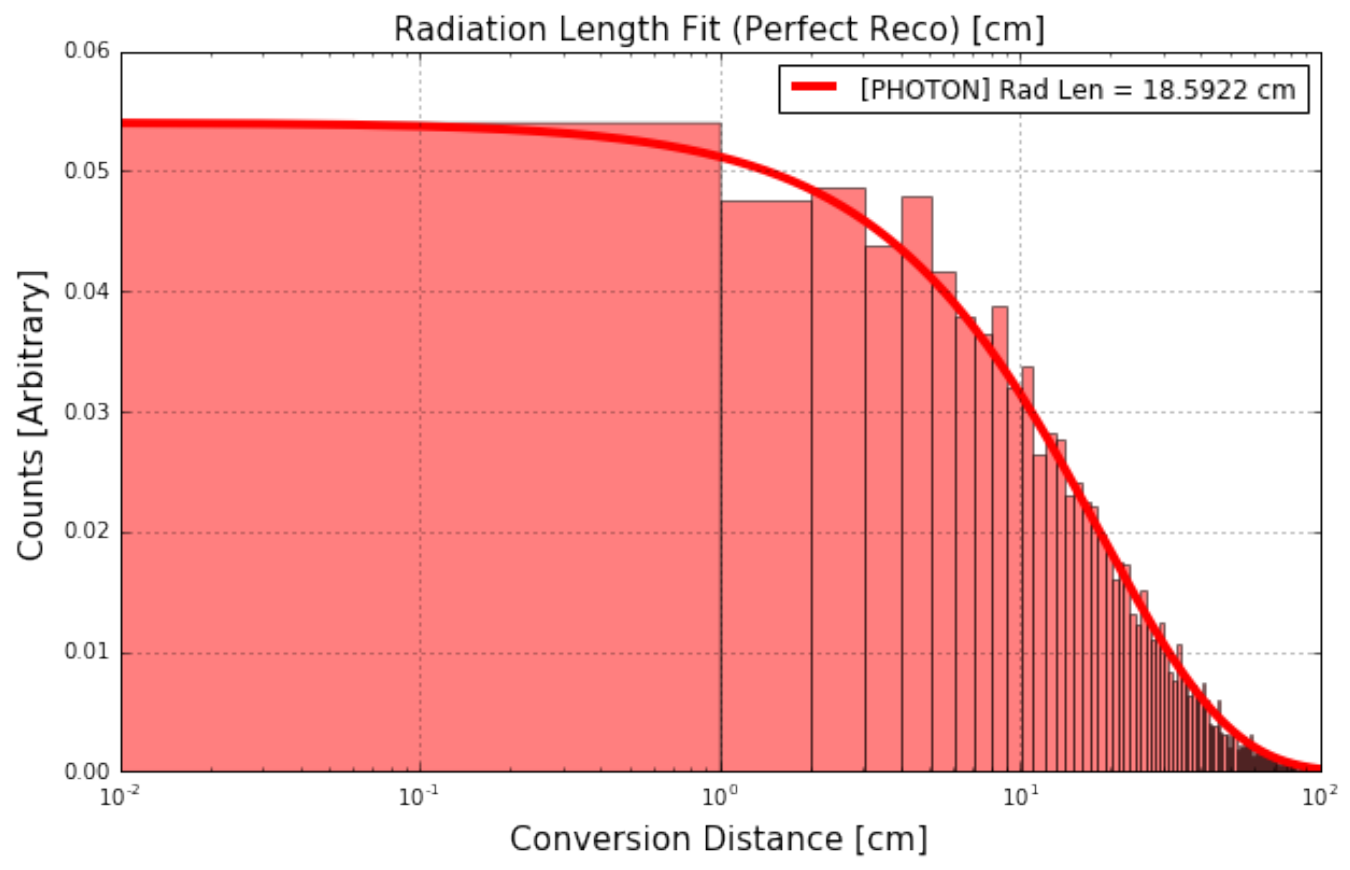

Figure 6.7: AlgoEMPart training results on perfect reconstructed photon showers as described in Section 6.3.1.1: Radiation length fit to single photon showers.

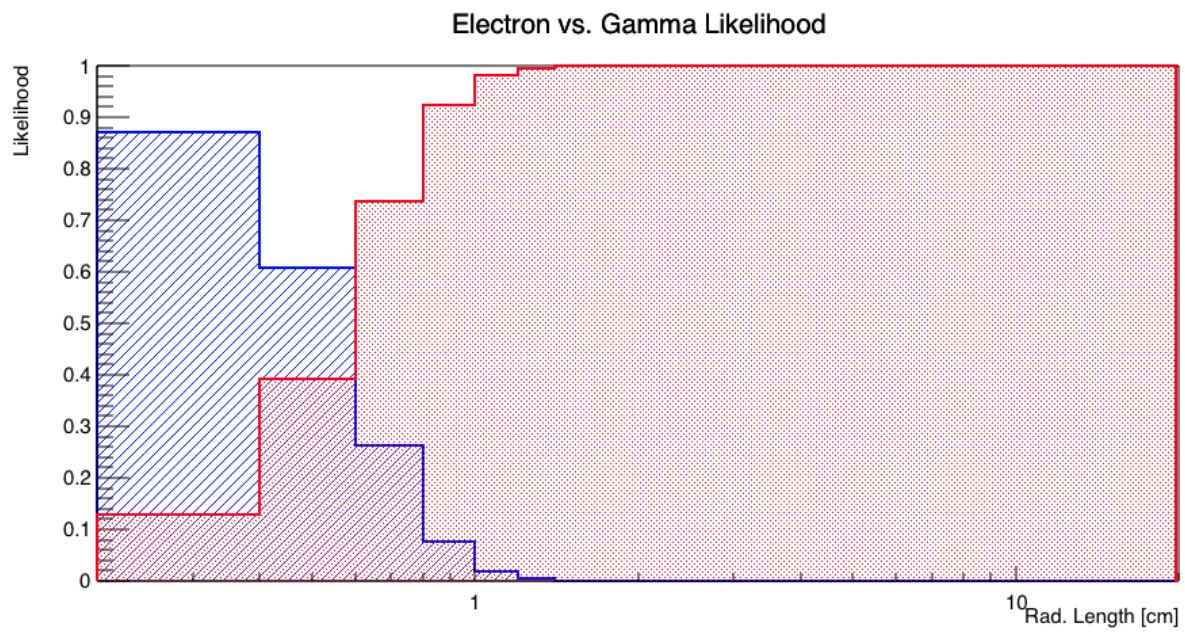

Figure 6.8: AlgoEMPart: Computed 1D likelihood vs conversion distance (integrated over all energies): red is photon, blue is electron. How the likelihood is computed is described in Section 6.3.1.1. 
analysis. The flowchart refers to determining if two showers are correlated and determining if a shower is correlated with the start of a track. A schematic which diagrams how these determinations are made is shown in Figure 6.10. A list of configurable parameters and their chosen cut values is shown in Table 6.2. A more detailed description of Figure 6.9 is given in the following paragraphs.

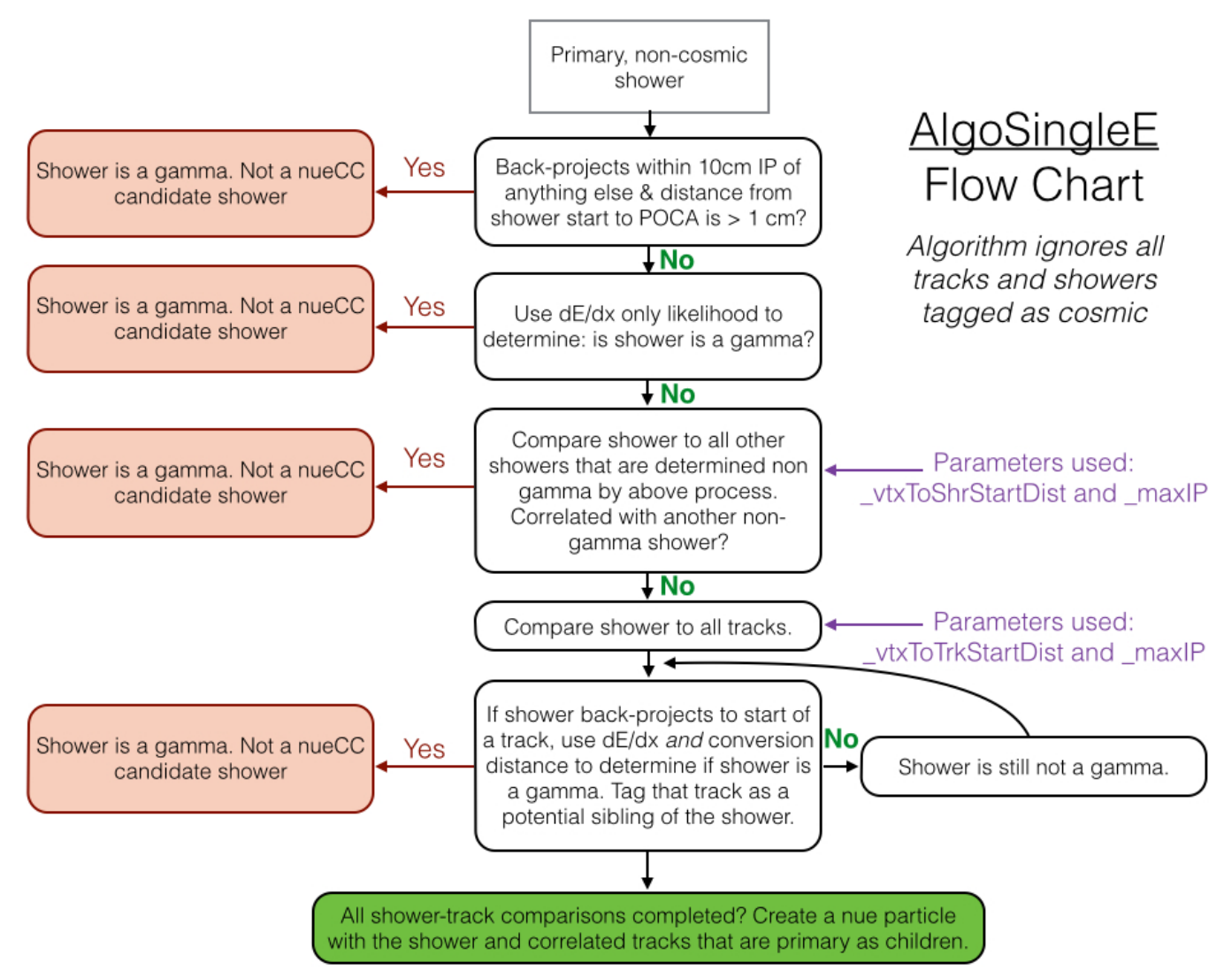

Figure 6.9: A flowchart depicting decisions the algorithm makes for each primary, noncosmic shower. If the algorithm gets to the bottom of the flowchart, that shower was determined to be from a $\nu_{e}^{C C}$ interaction, and a $\nu_{e}$ particle is created. For clarification of what some acronyms mean, see Figure 6.10.

To begin, the algorithm attempts to reject any showers which are highly likely to be photons. For each primary, non-cosmic shower ("shower A" in Figure 6.10), this algorithm computes a point of closest approach (POCA) and impact parameter (IP) between the 


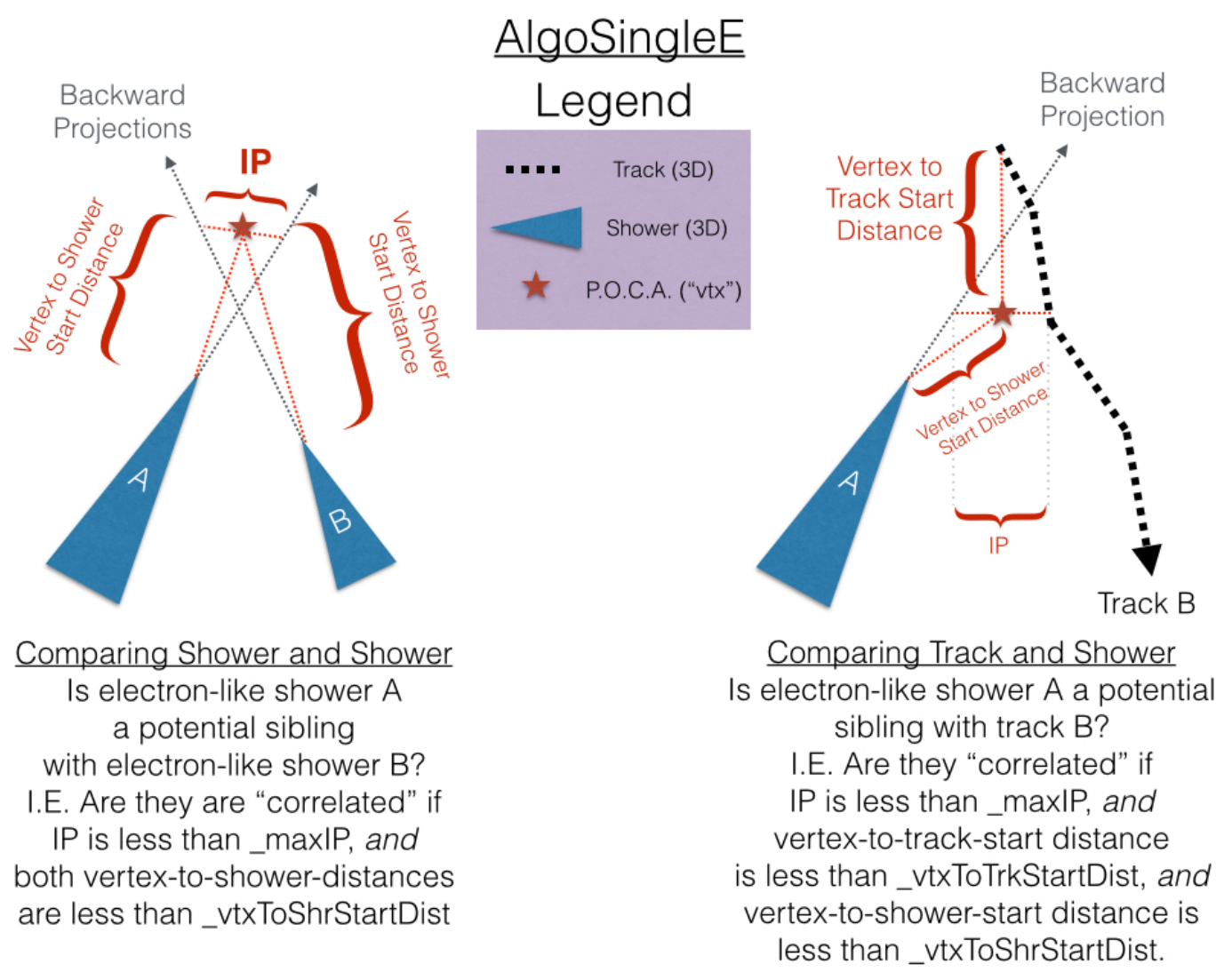

Figure 6.10: Schematic cartoons indicating how the signal selection algorithm makes decisions determining if two reconstructed showers are correlated, and if a reconstructed shower is correlated with a reconstructed track (as described in Figure 6.9.).

shower's back-projection and each other track. Additionally, the POCA and IP are computed between the shower's back-projection and each other shower axis back-projections. If the smallest impact parameter is less than 10 centimeters and the distance between the shower's start point and the point of closest approach is greater than 1 centimeter, the algorithm assumes this shower is a photon and it rejects the shower as a potential $\nu_{e}^{C C}$ shower, without any $\mathrm{dE} / \mathrm{dx}$ considerations. The value of 10 centimeters is chosen because such a displacement from the vertex would not occur for electron showers, even including reconstruction resolution effect:4. Otherwise, the algorithm continues by using AlgoEM-

\footnotetext{
${ }^{4}$ Note that for $\nu_{e}^{C C}$ interactions and "perfect reconstruction", these impact parameters and points of closest approach between the $\nu_{e}^{C C}$ electron shower and other tracks in the event are incredibly small (sub
} 
Part's trained likelihood function to determine based on $\mathrm{dE} / \mathrm{dx}$ alone whether this shower is gamma-like and should therefore be ignored.

Assuming this potential $\nu_{e}^{C C}$ shower has so far been found to be electron-like, the algorithm then compares the shower to all other showers in the event that are not marked as cosmic, and are not already reconstructed to be the descendant of another particl 5 . The purpose of this portion of the code is to enforce that the topology of interest includes a single electron exiting the neutrino interaction. If any other electron-like showers are nearby that could be potentially correlated, the shower is rejected as a candidate $\nu_{e}^{C C}$ electron.

The candidate shower is then compared to every track in the event that has not been tagged as cosmic to look for correlations between the potential $\nu_{e}^{C C}$ electron and tracks (Figure 6.10). The topology of interest allows for the electron to point back to the start of a track, but not to the middle or end of a track (as these showers are likely delta rays or muon decay Michel electrons). At this point, the code again uses a likelihood from AlgoEMPart to determine if the shower is still electron-like, this time using both the shower's $\mathrm{dE} / \mathrm{dx}$ and the distance between the shower start and the computed POCA as a radiation length. This is a more powerful discrimination to determine if the shower remains electron-like. If the shower remains electron-like and is found to be correlated with the start of a track, the track is stored as associated with another final state particle of the $\nu_{e}^{C C}$ interaction. The energy of this track will later be included in the reconstructed neutrino energy.

At this point, a $\nu_{e}^{C C}$ event has been found, and the shower and any associated tracks are stored to be included in the sensitivity analysis.

centimeter). The cut values are chosen to be much larger to simulate estimated automatic track and shower reconstruction resolutions.

${ }^{5}$ Cosmic tagging and descendant determination are done by upstream event reconstruction algorithms not described in this thesis for readability reasons. 


\begin{tabular}{|l|l|}
\hline \multicolumn{2}{|c|}{ AlgoSingleE: Parameters } \\
\hline Parameter Name & $\begin{array}{l}\text { Cut } \\
\text { Value }\end{array}$ \\
\hline \hline Use Radiation Length & True \\
\hline Max Vertex-to-Track-Start Distance & $1 \mathrm{~cm}$ \\
\hline Min Vertex-to-Shower-Start Distance & $50 \mathrm{~cm}$ \\
\hline Maximum IP & $1 \mathrm{~cm}$ \\
\hline
\end{tabular}

Table 6.2: The list of configurable parameters and their values used in the AlgoSingleE $\nu_{e}^{C C}$ signal selection algorithm. Note that for $\nu_{e}^{C C}$ interactions and "perfect reconstruction", these actual values the distance-based parameters represent are incredibly small (sub centimeter). The cut values are chosen to be much larger to simulate estimated automatic track and shower reconstruction resolutions.

\subsubsection{Configurable Parameters}

The configurable parameters for this algorithm are summarized in the Table 6.2. Note that with "perfect reconstructed" showers, these distances are always very small (less than 0.1 centimeters), so these values were chosen to introduce some realism into the algorithm by estimating detector resolutions.

\subsubsection{Energy Reconstruction}

With the candidate $\nu_{e}^{C C}$ interactions identified, the neutrino energy is reconstructed in two ways. First, the angle and energy of the selected $\nu_{e}^{C C}$ electron is used to compute an energy, $E_{\nu}^{C C Q E}$ from the CCQE formula, Equation 5.1. This is the same energy definition that is used in the MiniBooNE oscillation analysis. Note that this energy is only valid for truly $\nu_{e}^{C C Q E}$ interactions, which are a subset of the selected $\nu_{e}^{C C}$ sample. In addition, $E_{\nu}^{C C Q E}$ can be significantly effected by nuclear effects such as Fermi motion of nucleons in the target nucleus. 
An additional, more accurate energy estimation, $E_{\text {calo }}$ is calculated by looping over all particles tagged as descendants of the reconstructed $\nu_{e}$ and adding up their deposited energies. For $\nu_{e}^{C C}$ events, this amounts to adding the deposited energy of the electron shower, along with the deposited energies from all protons exiting the interaction vertex, and energies from charged and neutral pions exiting the vertex (adding the pion masses in the case of charged pions). Additionally the electron mass is added, though this changes the calculated energy negligibly. Plots describing the neutrino reconstruction performance for correctly identified $\nu_{e}^{C C Q E}$ interactions can be seen in Figures 6.11 and 6.12 . The energy resolution for true neutrino energies below $500 \mathrm{MeV}$ is on the order of $15 \%$, and the bias indicates that the energy reconstruction method tends to underestimate true neutrino energy by about $25 \%$. Note that this bias is computed as the mean reconstructed energy of all events in a specific true energy bin rather than the center of a Gaussian fit to the central distribution of events, so the bias is skewed down by outliers. In addition, the neutrino energy is generally underestimated both because energy lost to neutrons or showering particles is not included in the energy definition, and because neutrino-induced tracks are not associated with the interaction with perfect efficiency.

\subsection{Backgrounds}

\subsubsection{Background Topologies}

\subsubsection{Intrinsic $\nu_{e}$}

The intrinsic $\nu_{e}^{C C Q E}$ background comes from electron neutrinos originating from the portion of the Booster Neutrino Beam-line (BNB) flux from muon and kaon decays in the decay pipe. The topology of these events involve one electron in the final state, with any number of protons exiting the interaction vertex, but no pions or muons exiting the interaction vertex. Neutral particles included in hadronic activity are also ignored as they are invisible from the point of view of a LArTPC. This background is irreducible for a $\nu_{e}^{C C Q E}$ appearance search, and is the dominant background in this analysis. These intrinsic $\nu_{e}$ events in the relevant low energy region are mostly from muon decay in the beam-line. The flux uncertainty 


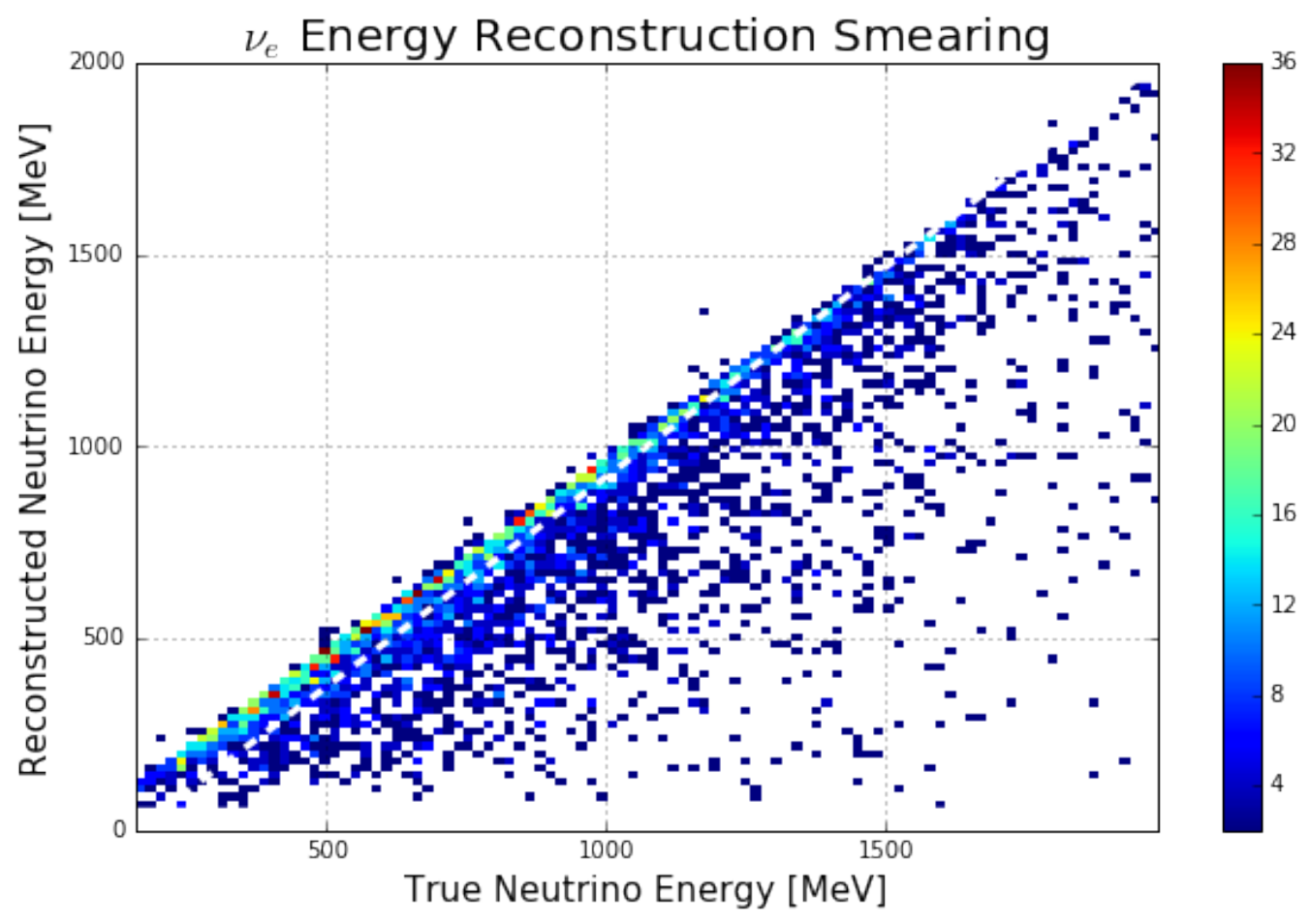

Figure 6.11: Reconstructed neutrino energy as described in Section 6.3.3 versus true neutrino energy. This plot was made from "perfect reconstruction" objects in correctly identified $\nu_{e}^{C C Q E}$ events after all final analysis cuts were placed.

can be constrained by a parallel $\nu_{\mu}$ analysis, as was done in MiniBooNE. The intrinsic $\nu_{e}$ events at higher energies mostly come from $K^{+}$decay in the beam-line, although $K^{+}$decay neutrinos also make significant contributions to electron neutrinos at lower energy. While constraining $\nu_{e}$ from muon decay will be done with a parallel $\nu_{\mu}$ analysis, constraining $\nu_{e}$ from kaon decay in the beam line is discussed in the next chapter of this thesis.

\subsubsection{Intrinsic $\nu_{\mu}$}

The intrinsic $\nu_{\mu}$ background comes from $\nu_{\mu}^{C C}$ interactions from BNB muon neutrinos. Despite the enormous ratio of $\nu_{\mu}^{C C}$ events to $\nu_{e}^{C C}$ events, this is a sub-dominant background in this analysis. Potential sources of mis-identifications (MIDs) must always involve at least one shower, and there should not be a track recognized as a muon exiting the interaction vertex. For this reason, most $\nu_{\mu}^{C C}$ MIDs are from either: 


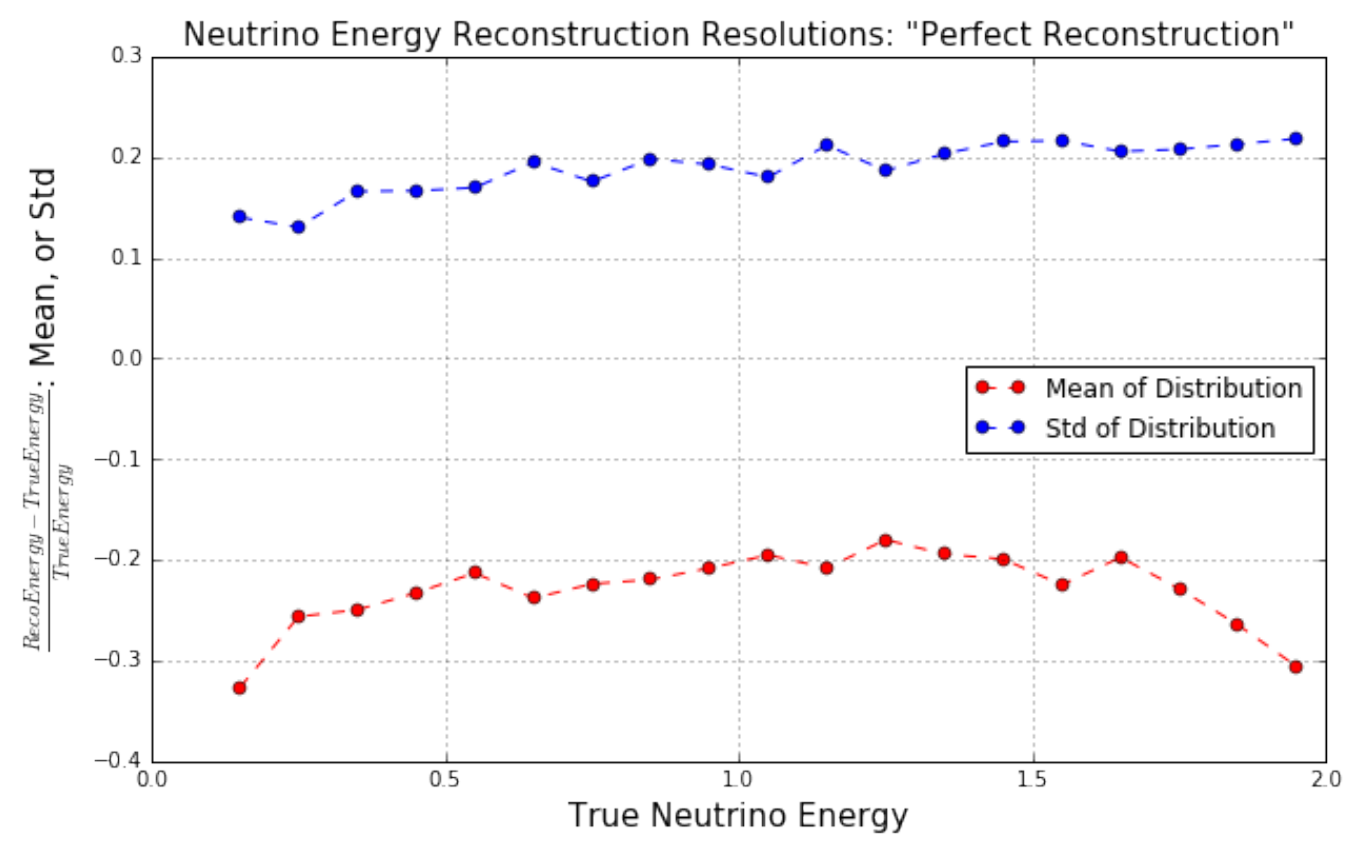

Figure 6.12: A neutrino energy resolution and bias plot. This is created by binning Figure 6.11 in true neutrino energy and making a distribution of (Reco Energy - True Energy)/(True Energy). For each bin, the mean (red) and standard deviation (blue) are plotted in the above figure. This plot was made from "perfect reconstruction" objects in correctly identified $\nu_{e}^{C C Q E}$ events after all final analysis cuts were placed.

1. $\mu$ decay electrons (either in flight, or at rest when the energy of the electron is in the very high end of the Michel spectrum, despite their energies being on the order of 50 $\mathrm{MeV})$, or

2. $\nu_{\mu}^{C C}$ events with a neutral pion in the final state.

The first MID source is suppressed because the electron points back to the end of the muon track and therefore is generally tagged by event selection algorithms. Additionally, these events are removed if the outgoing track is correctly identified as a muon. However, this background may be more prominent if the muon reconstructed track direction gets flipped (after which, correct identification of the muon track becomes more difficult, and the electron would point back to the start of the track). The effect of flipping this track is not included in this analysis, but the impact is expected to be small because there are several 
handles on track directionality in LArTPCs including from multiple Coulomb scattering, delta rays, and calorimetric information (the presence of a Bragg peak). Also, one can differentiate between such an electron-muon background event from an electron-proton signal event by leveraging the differing $\mathrm{dE} / \mathrm{dx}$ for the muon as compared to the proton.

The second MID source occurs when one of the photons of the neutral pion decay from a $\nu_{\mu}^{C C} \pi^{0}$ interaction is misidentified as an electron. This background is greatly suppressed because the $\mathrm{dE} / \mathrm{dx}$ of the shower should be photon-like, and the shower points back towards the muon start point. The fact that the shower is displaced from the muon start point allows for a photon-like likelihood calculation using both the shower $\mathrm{dE} / \mathrm{dx}$ and the radiation length, which is a more powerful discrimination tool to tag the shower as being from a photon (as described in Section 6.3.1). Additionally, if the second photon converts inside of the detector, this provides another handle that the shower is from a neutral pion. Lastly, if the muon is correctly identified as such (from $\mathrm{dE} / \mathrm{dx}$ or other considerations) then this background gets further reduced.

\subsubsection{Intrinsic Neutral Current (NC)}

The intrinsic NC background comes from neutral current interactions by any neutrino type from the beam. In these interactions, the neutrino interacts with the exchange of a neutral $Z$ boson, and the neutrino carries off some energy and momentum as it exits the detector. The predominant NC background for the low energy excess analysis are $\nu_{x}$ (mostly $\nu_{\mu}$ ) interactions with a neutral pion in the final state. This was by far the most dominant background in the MiniBooNE $\nu_{e}$ appearance analysis (see Figure 5.4). In this topology, one of the photons from the neutral pion decay is mis-identified as an electron coming from a $\nu_{e}^{C C}$ interaction. This background is significantly mitigated when both photons convert inside of the detector. In that case, the presence of two showers pointing back to a common origin allows for the event to be rejected.

An additional NC background topology is NC $\Delta \rightarrow N \gamma$, though this background is subdominant to the aforementioned neutral pion decays (with a relative rate of about $0.5 \%$ as 
compared to neutral pion decays).

\subsubsection{Beam-Induced, TPC External ("B.I.T.E.")}

This background comes from beam neutrino interactions that occur outside of the TPC sensitive volume, but inside of the cryostat volume (including the cryostat walls). By volume (and mass), this region is roughly half of the volume of the entire cryostat. The predominant topology for this background are neutrino interactions involving a neutral pion in the final state where only one photon from the pion decay converts inside of the active volume. Since this photon may not point back to any other reconstructed objects in the TPC, only its $\mathrm{dE} / \mathrm{dx}$ can be used in the electron/photon separation likelihood which provides less discrimination power than if a radiation length could be used as well. Note that this analysis does not explicitly ask for a visible hadronic vertex, otherwise many of these backgrounds would be mitigated (though much of the signal would be mitigated as well). In this analysis, this background can be mitigated, as was done in MiniBooNE, with cuts like backward-projected distance to a TPC wall, since they are all coming from outside of the TPC.

\subsubsection{Cosmic}

This background comes from cosmic rays that pass through the detector. The relatively high cosmic rate inside of the detector hall makes for on the order of tens of cosmic rays passing through the detector during the full readout window. Since the readout window is several milliseconds (since this is the time scale with which ionizations drift across the width of the TPC) and the cosmic rate through the detector is on the order of $5 \mathrm{kHz}$, roughly 20 cosmics arrive during each readout window. Measured reconstructed cosmics for an example full readout window of $4.8 \mathrm{~ms}$ are shown in Figure 6.13.

Cosmic MID topologies include but are not limited to showers that radiate off of cosmic ray muons, and showers born from cosmic neutron scatters. The vast majority of MIDs from cosmics can be removed by requiring the reconstructed neutrino interaction is matched to a flash inside of the beam gate window. Given the ratio of beam gate window size to 


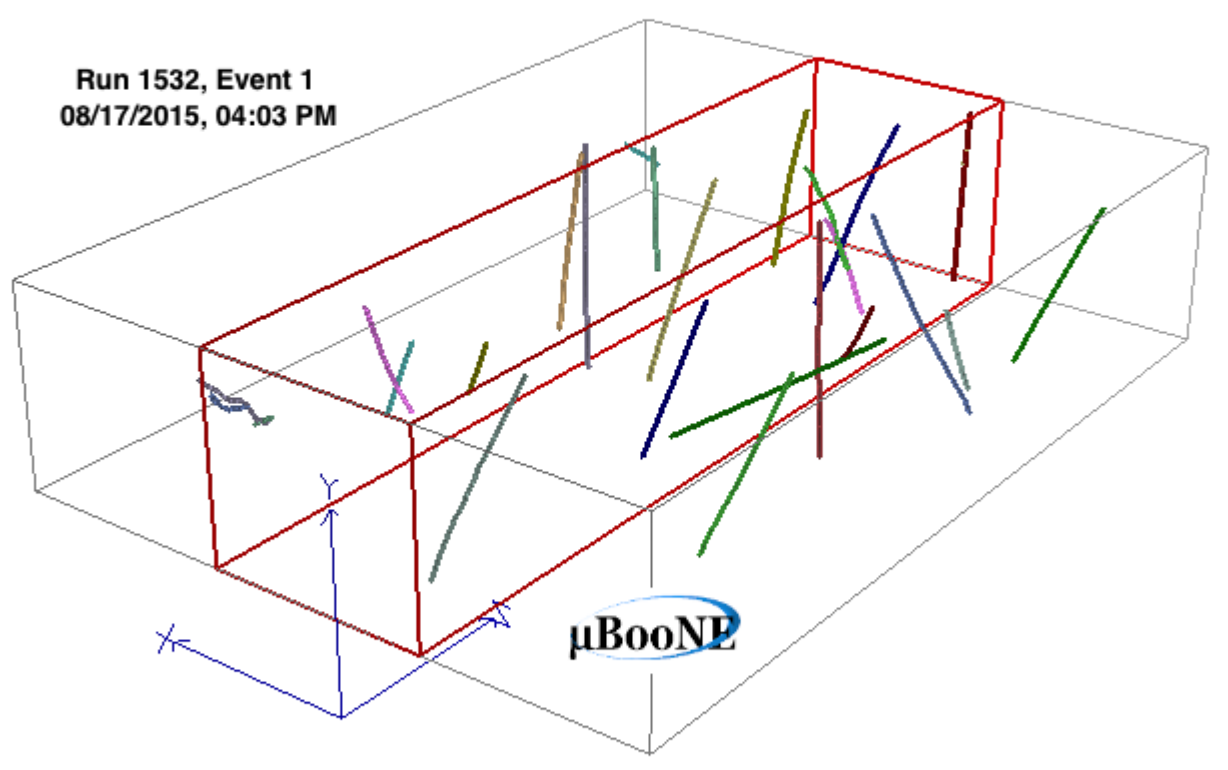

Figure 6.13: A 3D event display showing measured cosmic tracks entering the MicroBooNE detector. The three boxes show the full readout window of the detector which corresponds to $4.8 \mathrm{~ms}$. The red highlighted box shows the physical volume of the TPC. The colored lines are $3 D$ reconstructed cosmic tracks. Data taken in August, 2015.

readout window size $\left(\frac{1.6 \mu \mathrm{s}}{4.8 \mathrm{~ms}}=0.0003\right)$, this requirement mitigates a large fraction of the cosmic backgrounds. However, the majority of triggered beam events are not triggered by a neutrino interaction in them but are instead triggered by a cosmic ray that arrived during the beam gate window. These events are referred to as "in-time" cosmics. Given the number of readouts triggered by cosmics inside of the beam gate window rather than neutrinos arriving inside of the beam gate window, the cosmics background can be sizable, especially 
in the relevant low energy region. The measured relative rate of bright reconstructed flash times inside the $1.6 \mu \mathrm{s}$ beam spill window with respect to the constant cosmic background in MicroBooNE is shown in Figure 6.14. The ratio of cosmic-triggered events to neutrino triggered events from that plot is $1: 0.45$, and only about half of those neutrino interactions are inside of the active volume (and are reconstructible), making the ratio closer to 5 triggered cosmic readouts for every neutrino triggered readout in which the neutrino is reconstructible 6

In addition to these "in-time" cosmics (those that triggered a readout and arrived during the beam gate window), an additional cosmic background comes from events in which a neutrino interacts inside of the beam gate window, triggering a readout, but an out-of-time cosmic MID topology gets incorrectly matched to the neutrino flash. These cosmic MIDs are appropriately referred to as "out-of-time" cosmics. The "out-of-time" cosmic background is not included in this analysis, but its size relative to the "in-time" cosmics is small.

\subsubsection{Background Normalization}

As described in Section 6.2.1. the simulated background samples used in this analysis can be classified either as beam-induced, or cosmic. Each beam-induced background has an associated simulated protons-on-target (POT) generated, and they are each normalized to $6.6 \times 10^{20} \mathrm{POT}$, the nominal amount of beam scheduled to be delivered to MicroBooNE over the course of three years of running. The cosmic simulated sample does not have an associated POT, but instead has an associated total exposure time. The normalization of this sample must involve disregarding the event selection cut in which a reconstructed optical flash occurs within the timing of the beam gate window (described in Section 6.4.3).

\footnotetext{
${ }^{6}$ While this calculation assumes the optical flashes are bright (flashes greater than 50 photoelectrons), the triggering threshold is lower than that so this ratio estimate is conservative; in reality the ratio is larger than $5: 1$.
} 


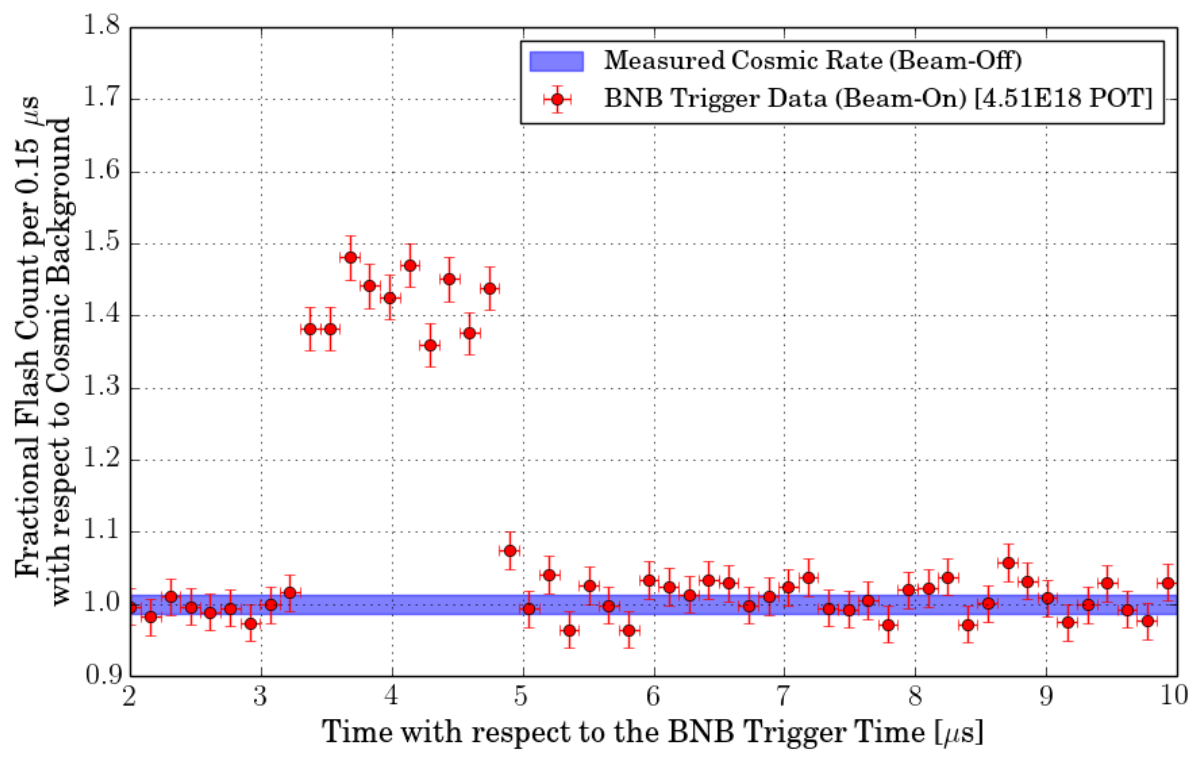

Figure 6.14: The measured distribution of flash times (requiring flashes greater than 50PE) with respect to the trigger time for BNB-triggered events, shown as a ratio to the expected cosmic rate from off-beam data. The blue band denoting the cosmic rate was centered at one, with a width corresponding to the measured uncertainty in the cosmic rate. A clear excess can be seen due to neutrinos between 3 and $5 \mu$ s after the trigger. This is where the neutrinos were expected based on the RWM signal arrival time. A total of 1.92E6 BNB triggered events (unbiased trigger) were used to produce this plot.

The total beam-gate-open exposure time corresponding to $6.6 \times 10^{20}$ POT is 211 seconds:

$$
\frac{6.6 \times 10^{20} P O T}{5 \times 10^{12} \frac{P O T}{\text { spill }}} \times 1.6 \frac{\mu s}{\text { spill }}=211 \text { seconds }
$$

Therefore, a simple cosmic background scale factor is computed based on the simulated cosmic exposure time corresponding to 211 seconds.

\subsubsection{Analysis Cuts and Results}

A number of additional analysis cuts are placed on the selected candidate $\nu_{e}^{C C}$ events. The purpose of these cuts is to first down-sample the selected $\nu_{e}^{C C}$ selected events into a sample of $\nu_{e}^{C C Q E}$ events by removing those reconstructed as having pions in the final state. An 
additional reason to place these cuts is to mitigate backgrounds that the event selection algorithms were unable to remove themselves. The analysis cuts used are described here.

The analysis cuts placed are:

1. The reconstructed $\nu_{e}$ interaction is matched to a flash inside of the beam gate window (this cut is not placed on the cosmic background simulated sample for reasons described in Section 6.4.2.

2. If the reconstructed $\nu_{e}$ interaction has additional particles reconstructed to be in the final state, those particles are limited to protons only (using assumed perfect efficiency to tag a track as a proton). This down-samples the $\nu_{e}^{C C}$ selected sample to specifically $\nu_{e}^{C C Q E}$.

3. Minimum primary $\nu_{e}^{C C}$ reconstructed electron energy deposited of $60 \mathrm{MeV}$.

4. A fiducial volume of $10 \mathrm{~cm}$ from all sides of the detector is placed on the neutrino interaction vertex.

5. A projected-backwards-distance-to-wall cut of $40 \mathrm{~cm}$ is placed on the primary $\nu_{e}^{C C}$ reconstructed electron. The projected-backwards-distance-to-wall cut is computed by back-projecting the reconstructed $\nu_{e}^{C C}$ electron along its shower axis until it intersects with the TPC boundaries. The distance between the electron start point and the wall intersection point is the distance on which the cut is placed. This cut removes photons that are coming into the detector from outside and, thus, will point back to the TPC boundaries.

The efficiency of the event selection algorithms with "perfect reconstruction" inputs to select signal events and to select background events ("MID efficiency") is summarized in Table 6.3. The numerator of this efficiency is the number of events tagged as $\nu_{e}^{C C Q E}$ candidate interactions and the denominator is the true number of events with the specified interaction type. The second column in the table does not include the additional final analysis selection cuts which are placed on the reconstructed interactions in the efficiency numerator, and the third column does. The background categories are described in more 


\begin{tabular}{|l|l|l|l|}
\hline \multicolumn{4}{|c|}{ Event Selection Efficiencies } \\
\hline True Event Type & Efficiency & $\begin{array}{l}\text { Efficiency Including } \\
\text { Analysis Cuts }\end{array}$ & Final Number of Events \\
\hline \hline$\nu_{e}^{C C Q E}$ & $82.09 \%$ & $61.42 \%$ & 425.4 \\
\hline$\nu_{\mu}^{C C}$ & $0.68 \%$ & $0.02 \%$ & 21.3 \\
\hline$\nu_{x} \mathrm{NC}$ & $0.86 \%$ & $0.13 \%$ & 50.6 \\
\hline B.I.T.E. & $1.07 \%$ & $0.05 \%$ & 92.5 \\
\hline Cosmic & $5.88 \%$ & $0.12 \%$ & 23.1 \\
\hline
\end{tabular}

Table 6.3: Event selection efficiencies on "perfect reconstruction". The right-most column indicates the final number of selected events including analysis cuts, normalized to the nominal MicroBooNE running period of $6.6 \times 10^{20}$ protons on target.

detail in Section 6.4.1. In the case of the cosmic background category, the denominator is the true number of events triggered by a cosmic arriving within the beam gate window that do not include a neutrino interaction. Note that efficiency to select $\nu_{e}^{C C Q E}$ events including all analysis cuts is $61 \%$ and albeit with "perfect reconstruction", it is still more than twice the MiniBooNE efficiency as was planned.

The backgrounds to the $\nu_{e}^{C C Q E}$ appearance search in MicroBooNE are shown in Figure 6.15, normalized to the nominal MicroBooNE expected POT, with statistical-only error bars shown. The event selection is described in Section 6.3, the background topologies described in Section 6.4.1, the relative normalization between samples described in Section 6.4.2, and the energy reconstruction described in Section 6.3.3.

\subsection{MiniBooNE Low Energy Excess Signal Modeling In Mi- croBooNE}

This section describes how the signal sample is generated for this sensitivity study, along with the necessary assumptions made in the process. First, the signal is assumed to originate from beam-induced $\nu_{e}^{C C Q E}$ interactions (this is the electron-like hypothesis for the 


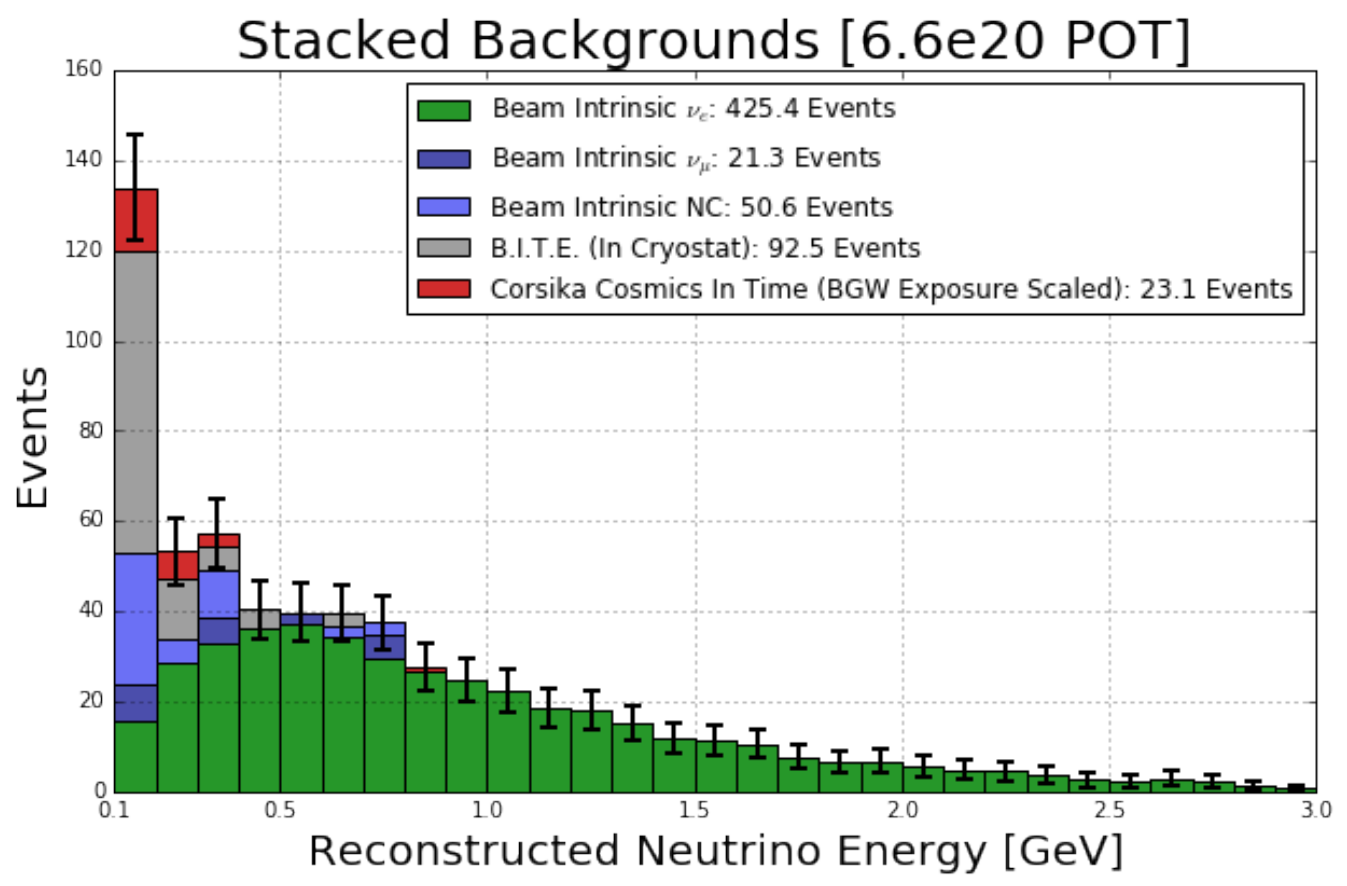

Figure 6.15: The backgrounds to the $\nu_{e}^{C C Q E}$ appearance search in MicroBooNE with statistical-only error bars shown. The event selection is described in Section 6.3, the background topologies described in Section 6.4.1, the relative normalization between samples described in Section 6.4.2, and the energy reconstruction described in Section 6.3.3.

excess). No study for a photon-like excess is described in this thesis. In this electron-like excess sensitivity estimate, the signal sample consists of simulated intrinsic $\nu_{e}^{C C Q E}$ interactions from the BNB generated uniformly throughout the MicroBooNE TPC. The energy and angle of these events are re-weighted to match the published energy and angle distributions of the excess as observed by MiniBooNE.

The MiniBooNE public data set 29] provides one dimensional distributions of $u_{z}, E_{v i s}$, and $E_{\nu}^{C C Q E}$ for the excess events, where $u_{z}$ is the z-direction cosine (the z- component of the unit momentum) of the observed particle in the low energy excess sample, $E_{v i s}$ is the visible energy associated with the event, and $E_{\nu}^{C C Q E}$ is the calculated neutrino energy assuming the interaction was charged current quasi-elastic (see Equation 5.1). 
Given these three one-dimensional distributions, a two-dimensional distribution of $u_{z}$ vs. $E_{v i s}$ is built by using the CCQE formula 7 . By comparing the MicroBooNE signal sample two-dimensional histogram of $\nu_{e}^{C C Q E}$ electron $u_{z}$ vs. $E_{v i s}$ to this MiniBooNE excess distribution, re-weighting factors are computed to reshape the MicroBooNE signal sample to match the MiniBooNE excess in this parameter space.

The strategy to generate the MiniBooNE excess two dimensional distribution is as follows:

1. Draw independently from each of the the two one-dimensional histograms: $u_{z}$ and $E_{\text {vis. }}$

2. For every drawn pair, calculate the corresponding $E_{\nu}^{C C Q E}$ with Equation 5.1 and decide whether to accept it according to the published MiniBooNE excess $E_{\nu}^{C C Q E}$ one-dimensional distribution.

- The probability of accepting a calculated $E_{\nu}^{C C Q E}$ that falls within the bin's boundaries is equal to the height of the unit-normalized $E_{\nu}^{C C Q E}$ distribution in any given bin.

3. Repeat this process until the number of accepted pairs $=\mathrm{N} \times$ Integrated number of excess events in the $u_{z}$ distribution (choosing $\mathrm{N}$ to be large: E.G. $\mathrm{N}=1000$ ). This two dimensional distribution represents the observed excess as seen from MiniBooNE.

4. Divide this distribution by the MiniBooNE published efficiency for single electrons (given as a function of $E_{v i s}$ ) to uncover the shape of the true excess event distribution in MiniBooNE8

5. Smooth the resulting two-dimensional distribution with a default ROOT TH2::Smooth() function.

\footnotetext{
${ }^{7}$ The CCQE energy formula should use lepton energy, $E_{e}$, but here $E_{v i s}$ is used instead. This is assuming that the lepton energy is approximately the same as the visible energy.

${ }^{8}$ No efficiency as a function of any other variable (E.G. $u_{z}$ ) has been published by MiniBooNE.
} 
The resulting two-dimensional distribution of $u_{z}$ vs. $E_{v i s}$ for the true MiniBooNE excess events is shown in Figure 6.16 .

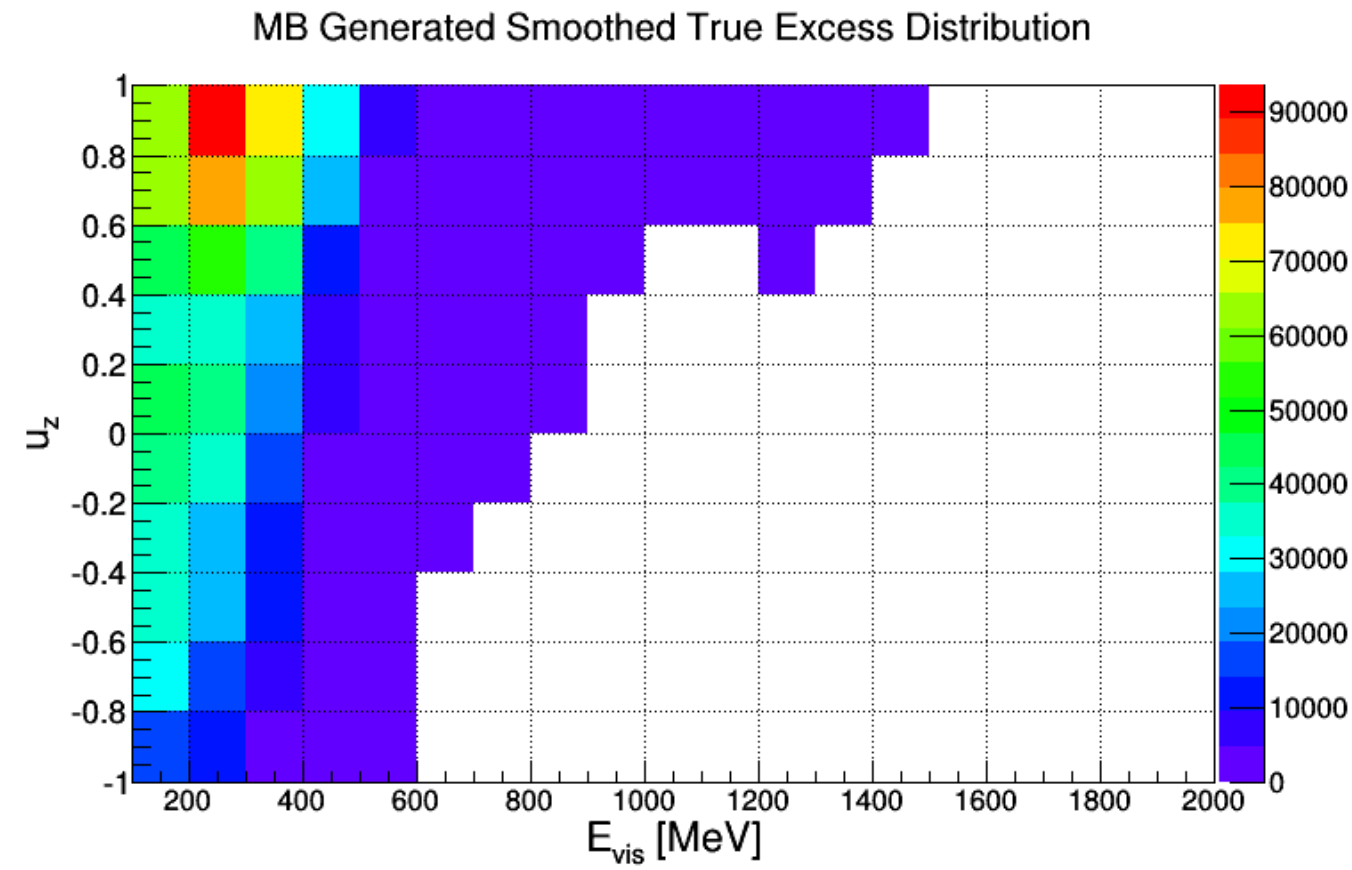

Figure 6.16: The computed distribution of $u_{z}$ (how forward-going the event is) vs. $E_{\text {vis }}$ for $N=1000$ times the MiniBooNE low energy excess (raw) events.

While the shape of the simulated MicroBooNE signal events is determined by the above process, the absolute normalization of this sample is computed by comparing the relative size of the signal with respect to the intrinsic $\nu_{e}$ backgrounds as observed by MiniBooNE. This is appropriate to do only because of the assumption that the origin of the low energy excess is intrinsic BNB $\nu_{e}$. From MiniBooNE data and MC, there are 187.7 excess signal events and 148.4 intrinsic $\nu_{e}$ events in the $E_{\nu}^{C C Q E}$ energy range from 100 to $600 \mathrm{MeV}$. In this analysis, the number of intrinsic $\nu_{e}$ events in that $E_{\nu}^{C C Q E}$ energy range in MicroBooNE is computed to be 159.8, and therefore the simulated signal sample is normalized to have $159.8^{*}(187.7 / 148.4)=201.3$ events in that $E_{\nu}^{C C Q E}$ energy range. 


\subsubsection{Sensitivity Results}

Shown in Figure 6.17 is the previously shown stacked backgrounds, now with the scaled MiniBooNe low energy excess signal included. Also labeled on the plot is the computed significance of $11.57 \sigma$, including only statistical errors. The details on the computation of the significance is described in the next section.

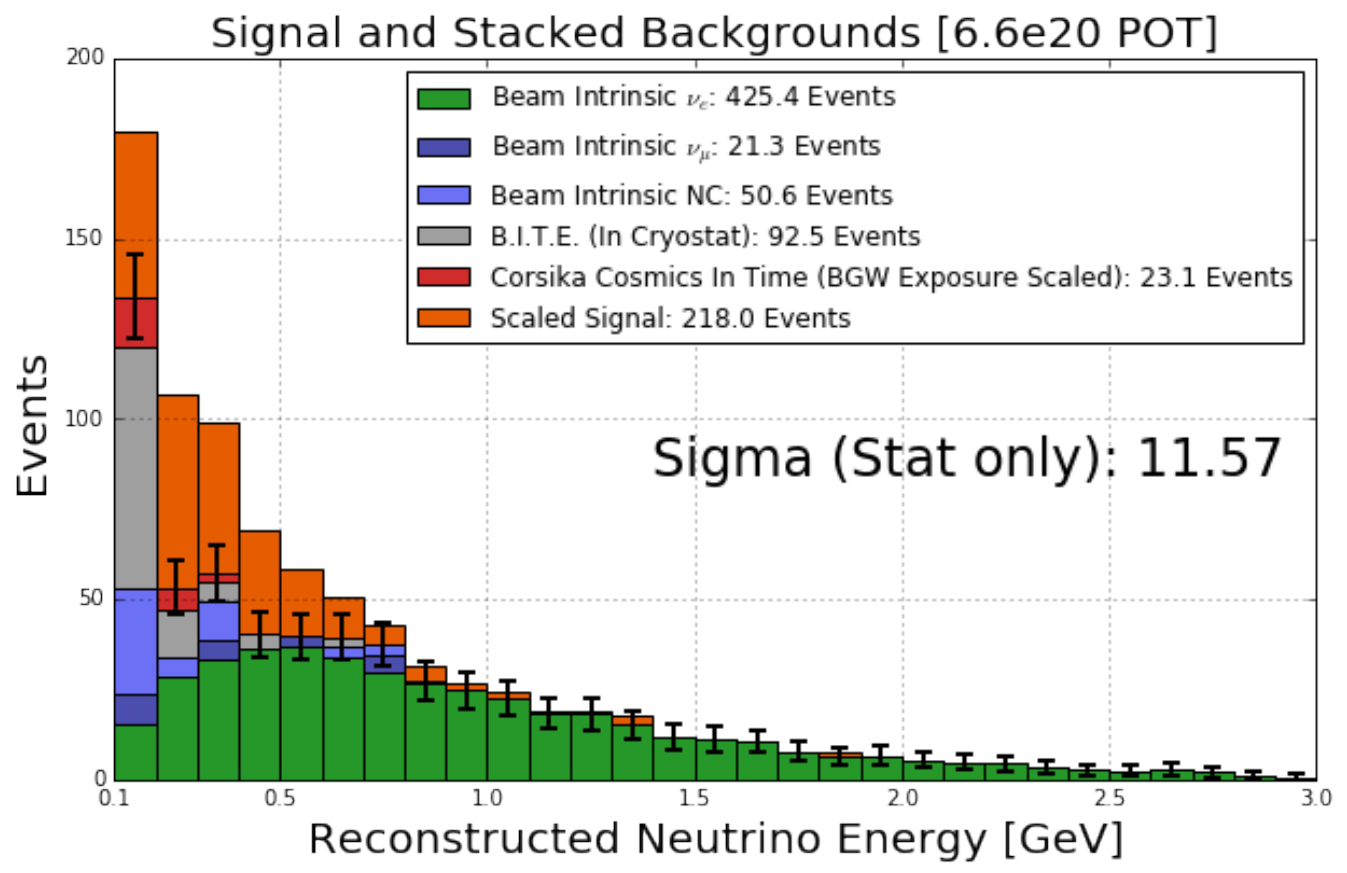

Figure 6.17: The backgrounds to the $\nu_{e}^{C C Q E}$ appearance search in MicroBooNE with scaled signal drawn. The event selection is described in Section 6.3, the background topologies described in Section 6.4.1, the relative normalization between samples described in Section 6.4.2, and the energy reconstruction described in Section 6.3.3. The scaled signal is described in Section 6.5.

\subsubsection{Significance Calculation}

For a given stacked background plot with a scaled signal on top, the statistical-only significance is can be computed with a chi-square test statistic as:

$$
\sigma=\sqrt{\Delta \chi^{2}}=\sqrt{\vec{S}^{T} E^{-1} \vec{S}}
$$


where $\vec{S}$ is a vector containing the size of the signal, with length equal to the number of bins in the histogram, $n$, and $E$ is the $n \times n$ statistical-only covariance matrix (diagonal matrix with entries equal to the summed number of background events per bin).

\subsubsection{Systematic Error Estimates}

In order to compute a realistic significance to the low energy excess in this analysis, a treatment of systematic uncertainties is necessary. While this is a detailed and important study, a conservative simplified estimation of these uncertainties is described here.

Previous studies described in the proposal for the Short Baseline Neutrino experiment (which includes MicroBooNE as one of three detectors) 30 quote the integrated $\nu_{e}$ and $\nu_{\mu}$ flux uncertainties to be on the order of 10 to $15 \%$. This number comes from systematic uncertainty estimates related to primary production of $\pi^{+}, \pi^{-}, K^{+}, K^{-}$, and $K_{L}^{0}$ in $p+B e$ collisions at $8 \mathrm{GeV}$ in the $\mathrm{BNB}$, secondary interactions of $p, n, \pi^{ \pm}$in the beryllium target and aluminum horn, and beam focusing with the magnetic horn.

The SBN studies also quote the cross section uncertainties to be on the order of $20 \%$. This number comes from varying model parameters in the GENIE neutrino interaction generator to determine $1 \sigma$ uncertainties. Parameters varied include axial mass for CCQE events, axial mass for $\mathrm{CC}$ resonant neutrino production, axial mass for NC resonant production, neutral current normalization, and switching of deep inelastic scattering nuclear models. A full list of parameters varied and their $1 \sigma$ uncertainties can be found in the GENIE manual, Section 8.1 25.

While many of these uncertainties are strongly correlated across energy bins, a very conservative approach is to simply include a flat, uncorrelated flux uncertainty of $15 \%$ and a similarly flat, uncorrelated cross section uncertainty of $20 \%$. These uncertainties are applied to all beam-induced backgrounds in this analysis. The systematic uncertainties on the cosmic-induced backgrounds are not known, but given the relatively small size of these backgrounds in this analysis, even a systematic of $50 \%$ would have small impact on the 
final computed significance, so this systematic is neglected. Additional uncertainties from sources including detector systematics are expected to be sub-dominant to the relatively large flux and cross-section systematics, and are therefore similarly neglected. It is important to note that these conservative estimates are ignoring many in situ measurements that can be done in MicroBooNE to constrain the uncertainties, as MiniBooNE did. Doing a combined $\nu_{e}$ plus $\nu_{\mu}$ analysis highly constrains the flux ans cross-section systematics and reduces them considerably. Such a constraint is done with a combined error matrix which has off-diagonal elements relating the $\nu_{e}$ and $\nu_{\mu}$ event numbers. The $\nu_{e}$ cross-section uncertainties are constrained by the observed $\nu_{\mu}$ events since $\nu_{e}$ and $\nu_{\mu}$ cross-sections are the same except at very low neutrino energies where mass effects become relevant, but these are theoretically well understood. Also, $\mathrm{NC} \pi^{0}$ measurements help constrain cross section $\times$ flux uncertainties for that process, but this background should be small in any case for the MicroBooNE measurement.

In order to include the flux and cross-section systematics in the significance calculation, Equation 6.4 is modified. As quoted, $E$ is the $n \times n$ diagonal covariance matrix with entries equal to the summed number of background events per bin. To include the flux and cross-section systematics, $E$ is modified as

$$
E=E_{\text {stat }}+E_{\text {flux }}+E_{\mathrm{xsec}}
$$

where $E_{\text {stat }}$ is the previous statistical-only covariance matrix, while $E_{\text {flux }}$ represents the assumed uncorrelated flux systematic and $E_{\mathrm{xsec}}$ the assumed uncorrelated cross section systematic. $E_{\text {flux }}$ is diagonal with entries equal to the number of beam-induced (non-cosmic) background events in that bin times the fractional uncertainty, squared:

$$
E_{\text {flux }}^{i, i}=\left(N_{\text {beam-induced }}^{i} * 0.15\right)^{2}
$$

and $E_{\mathrm{xsec}}$ is similarly diagonal:

$$
E_{\mathrm{xsec}}^{i, i}=\left(N_{\text {beam-induced }}^{i} * 0.20\right)^{2}
$$




\subsubsection{Realistic Shower Reconstruction Efficiency}

So far in this analysis, the shower reconstruction efficiency has been $100 \%$ because the input objects are "perfect reconstruction". In order to emulate a more realistic potential shower reconstruction efficiency, an additional study has been done in which the shower reconstruction efficiency is modeled in an energy dependent way, though the efficiency is effectively constant at $85 \%$ for showers with energy included in this analysis (above 60 $\mathrm{MeV}$ deposited). The value of $85 \%$ was chosen because this is approximately the maximum shower efficiency for electromagnetic showers with energy between $50 \mathrm{MeV}$ and $5 \mathrm{GeV}$ published by the ICARUS collaboration [31]. The resulting stacked background histogram with modeled signal is shown in Figure 6.18. The computed (statistical only) significance when realistic shower reconstruction efficiencies are included in the analysis is $10.10 \sigma$.

Note that in comparing Figure 6.17 to Figure 6.18, not all sample sizes are simply reduced by $15 \%$. For example, the intrinsic NC backgrounds actually increase when the shower reconstruction efficiency is decreased. This is because these backgrounds arise pri-

marily from $\pi^{0}$ decays, and they are mitigated by event selection algorithms that locate both subsequent photon showers pointing back to a common origin and correlate them together. With reduced shower reconstruction efficiency, sometimes only one of the $\pi^{0}$ decay showers gets reconstructed and subsequently gets misidentified as a candidate $\nu_{e}^{C C}$ electron.

\subsubsection{Final Results}

A complete summary of MicroBooNE's computed significance to the MiniBooNE low energy excess assuming an electron-like signal hypothesis for "perfect reconstructed" objects, "perfect reconstructed objects" with simulated realistic shower reconstruction efficiency, with and without conservative systematic uncertainties is shown in Table 6.4.

\subsubsection{Next Steps}

The described "perfect reconstruction" sensitivity study to an electron-like excess is an important tool for MicroBooNE. The event selection algorithms have been developed with these objects, and are ready to be used out-of-the-box when automated track and shower reconstruction efforts become more fruitful in the future. Already the algorithms I have 


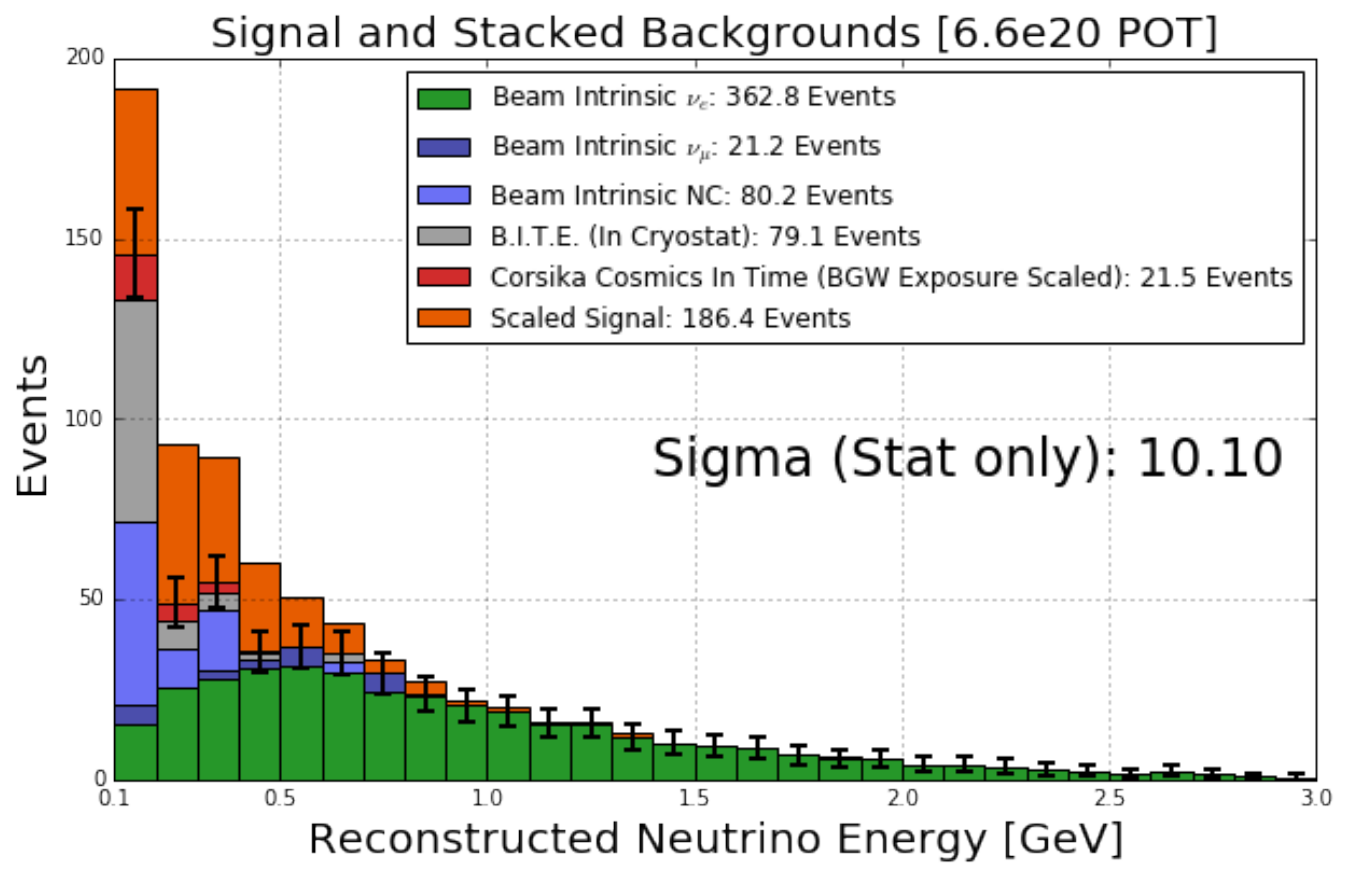

Figure 6.18: The backgrounds to the $\nu_{e}^{C C Q E}$ appearance search in MicroBooNE with scaled signal drawn. The event selection is described in Section 6.3, the background topologies described in Section 6.4.1, the relative normalization between samples described in Section 6.4.2, and the energy reconstruction described in Section 6.3.3. The scaled signal is described in Section 6.5. Here the shower reconstrution efficiency has been decreased from the "perfect reconstruction" value of $100 \%$ to $85 \%$ to emulate possible realistic shower reconstruction efficiencies.

developed are being used in combination with samples of MC simulation analyzed with full reconstruction programs to make a better sensitivity estimate. Efforts to apply systematic errors to this full reconstruction estimate are also in the process of being implemented. This sensitivity study can be used to set goals for the experiment by smearing or modifying the "perfect reconstruction" objects. For example, with this analysis the sensitivity to the low energy excess can be computed as a function of shower reconstruction efficiency to drive the shower reconstruction efforts. Also, the sensitivity as a function of POT delivered has 


\begin{tabular}{|l|l|l|}
\hline \multicolumn{3}{|c|}{ MicroBooNE Sensitivity Significances } \\
\hline "Perfect Reco" (stat only) & "Realistic Reco" (stat only) & "Realistic Reco" (stat+sys) \\
\hline \hline $11.57 \sigma$ & $10.10 \sigma$ & $6.52 \sigma$ \\
\hline
\end{tabular}

Table 6.4: Summary of computed significances to the MiniBooNE low energy excess in MicroBooNE assuming an electron-like signal hypothesis.

already been used to guide discussions on data blinding?

As mentioned, no photon-like excess search is described in this thesis. It is important to determine a sensitivity to an electron-like excess as well as a photon-like excess, because the true origin of the excess is still unknown. In order to conduct a photon-like excess search, event selection algorithms and analysis cuts would have to be developed to search for single photon events rather than single electron events.

An important takeaway from this electron-like excess analysis is that systematics play an important role in the ultimate sensitivity. The flux systematic is particularly large, but is constrained by a $\nu_{e}$ and $\nu_{\mu}$ combined analysis. As shown, the largest background in the MicroBooNE low energy excess search is from intrinsic $\nu_{e}$ in the beam, which come from either muon decay in the beam-line, or kaon decay in the beam-line. In the low energy region, the majority of intrinsic $\nu_{e}$ come from muon decay, which is tied to the observed $\nu_{\mu}$ events, as demonstrated by MiniBooNE. Constraining the remaining $\nu_{e}$ from kaon decay can be done by observing the high energy $\nu_{\mu}$ rate, the topic of the next chapter.

\footnotetext{
${ }^{9}$ The MicroBooNE Collaboration allows for a small sample (about 7.5\%) of its nominal collected neutrino data to be available to analyzers for reconstruction efforts and data to simulation comparisons. Developing analyses on a small subsample of the data (the "unblinded" portion) and then executing the analysis on the full data set only after it has been thoroughly vetted is important to prove that the analysis isn't biased towards a particular result.
} 


\section{Chapter 7}

\section{Studies of Kaons Produced at the Proton Target}

This chapter of the thesis will describe a MicroBooNE analysis done with the goal of constraining the intrinsic $\nu_{e}$ background for the previously described low energy excess search. As shown, this is the dominant background in the search. Over the entire booster neutrino beam energy range, $38 \%$ of the intrinsic $\nu_{e}$ background comes from kaon decay in the booster neutrino beam-line. The breakdown of $\nu_{e}$ interactions from the beam by parentage is shown in Figure 7.1. The systematic uncertainty on the flux of $\nu_{e}$ from kaon decay is one of the largest uncertainties reducing the low energy excess significance; this uncertainty was set to $40 \%$ in initial MiniBooNE publications, which is discussed in more detail in Section 7.1. This chapter will describe how measuring the highest energy $\nu_{\mu}$ interactions in MicroBooNE provides information about the kaon production in the beam-line, and constrain this important systematic. Exactly how these events are reconstructed and selected will be described, data to Monte Carlo comparisons will be shown, and the future prospects and next steps for this analysis will be discussed.

\subsection{BNB Kaon Production Introduction and Motivation}

As described in Chapter 4 , the BNB is predominantly composed of $\nu_{\mu}(92.9 \%)$ and $\bar{\nu}_{\mu}(6.5 \%)$ with a small contamination of $\nu_{e}$ and $\bar{\nu}_{e}(0.6 \%$ combined $)$. The BNB flux by neutrino type 


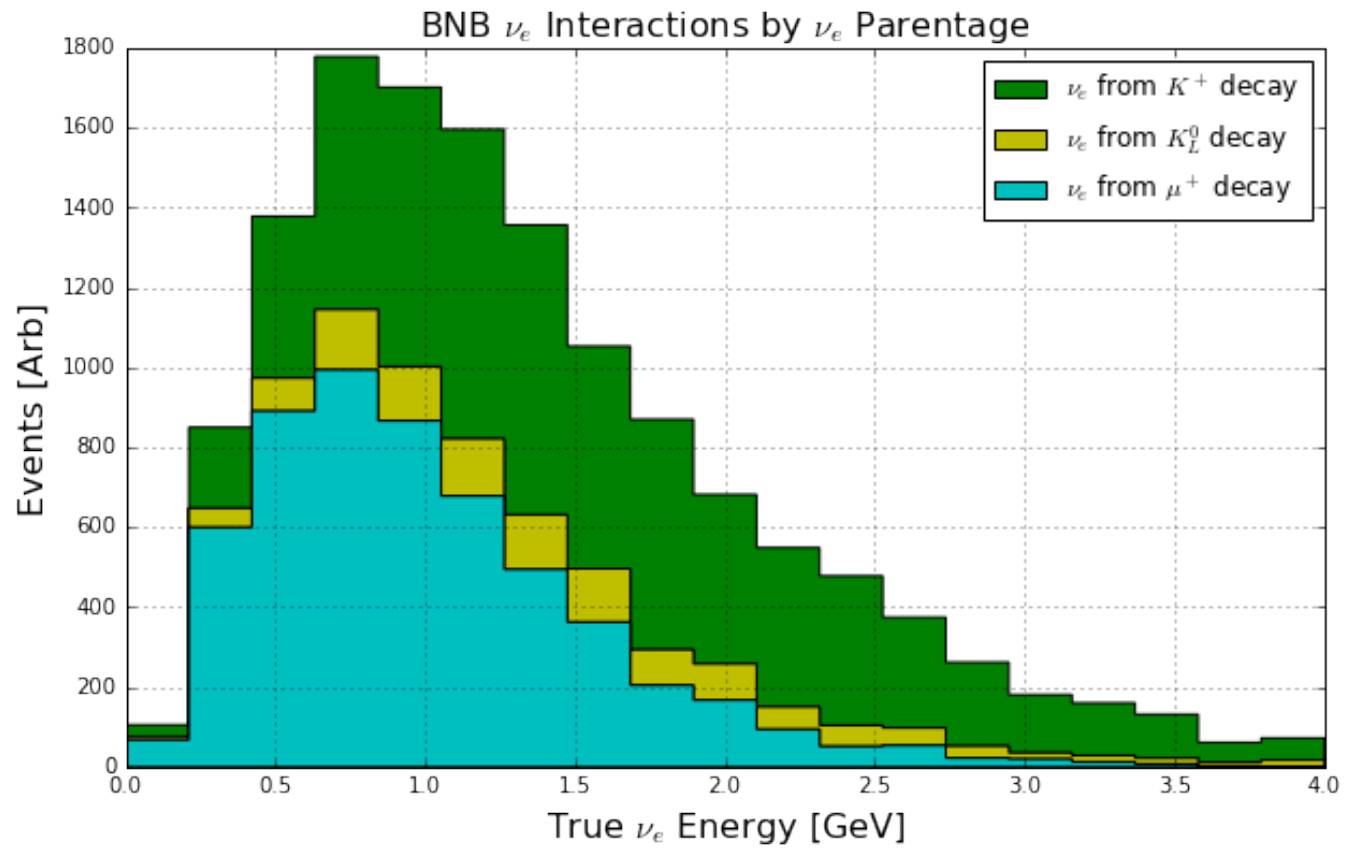

Figure 7.1: The breakdown of $\nu_{e}^{C C}$ interactions in MicroBooNE from the booster neutrino beam-line by $\nu_{e}$ parentage. $\nu_{e}$ from $K^{+}$decay is the primary contributor to the intrinsic $\nu_{e}$ interaction rate at higher neutrino energies.

at the MicroBooNE detector in neutrino-mode running is shown in Figure 7.2 .

The decay modes producing beam $\nu_{\mu}$ and $\bar{\nu}_{\mu}$ are

- $\pi^{+} \rightarrow \mu^{+}+\nu_{\mu}$

- $K^{+} \rightarrow \mu^{+}+\nu_{\mu}$

- $\pi^{-} \rightarrow \mu^{-}+\bar{\nu}_{\mu}$

and the decay modes producing beam $\nu_{e}$ and $\bar{\nu}_{e}$ are

- $K^{+} \rightarrow \pi^{0}+e^{+}+\nu_{e}$

- $\mu^{+} \rightarrow e^{+}+\bar{\nu}_{\mu}+\nu_{e}$

- $K_{L}^{0} \rightarrow \pi^{-}+e^{+}+\nu_{e}$

- $K_{L}^{0} \rightarrow \pi^{+}+e^{-}+\bar{\nu}_{e}$. 


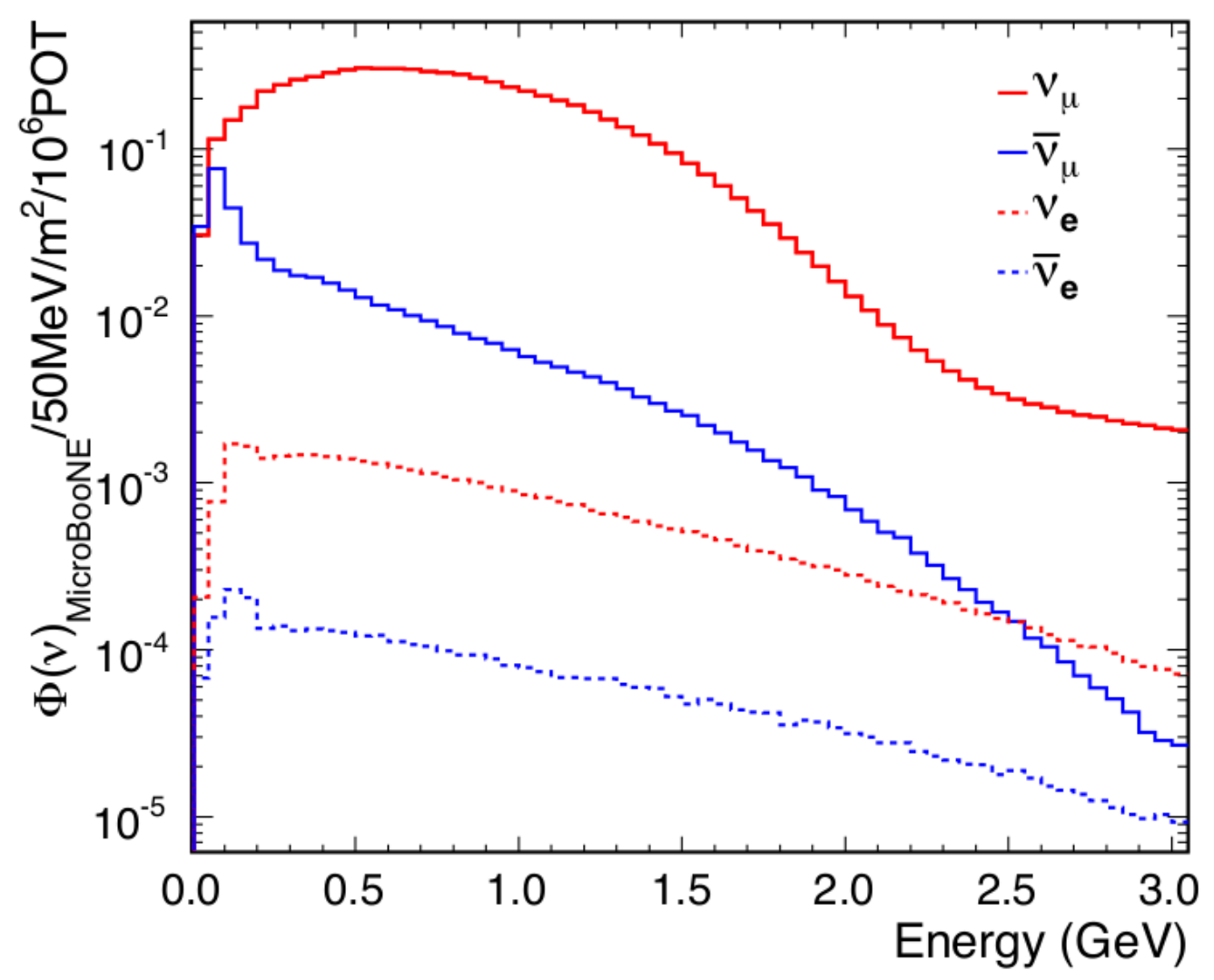

Figure 7.2: The Booster Neutrino Beam (BNB) flux at MicroBooNE.

For the $\nu_{\mu}$ in the beam, the breakdown of $\nu_{\mu}$ production parentage as a function of neutrino energy is shown in Figure 7.3. The two peaks in the $K^{+}$curve (red) at neutrino energies below $0.3 \mathrm{GeV} E_{\nu}$ are from two- and three-body $K^{+}$decays at rest. In the energy region $E_{\nu_{\mu}}<2 \mathrm{GeV}$, most $\nu_{\mu}$ come from $\pi^{+}$decay, whereas at higher energies most $\nu_{\mu}$ come from $K^{+}$decay. Therefore, the strategy to measure $K^{+}$production in the beam is to select high energy $\nu_{\mu}$ interactions and compare simulation to data in terms of event rates in order to compute a normalization factor with an uncertainty.

In the initial MiniBooNE publications, the systematic uncertainty on the intrinsic $\nu_{e}$ from $K^{+}$decay in the beam was $40 \%$. This systematic came from several sources in the beam simulation process including proton delivery/optics, secondary particle productions, hadronic interactions in the target or horn, and horn magnetic field. In contrast to kaon 


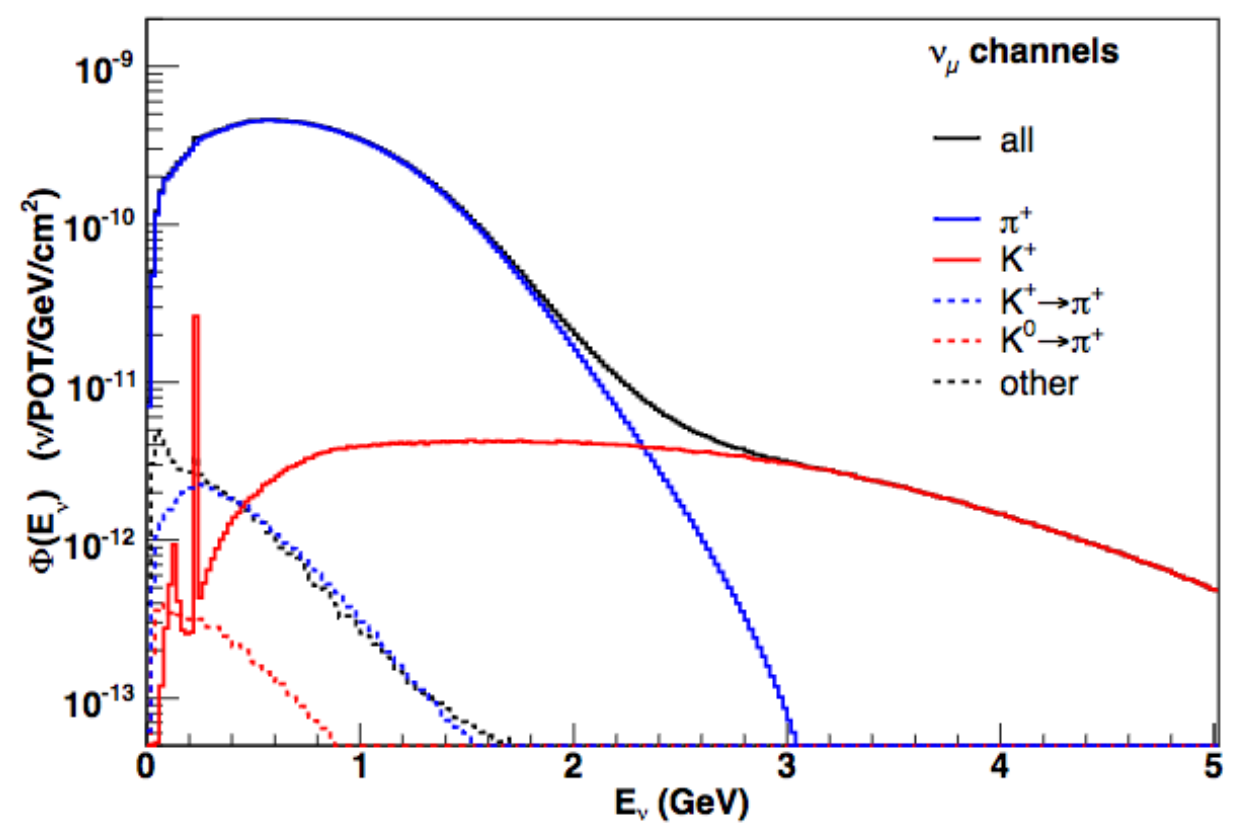

Figure 7.3: The beam $\nu_{\mu}$ parentage as a function of true neutrino energy.

production, the uncertainty for pion production is small since it was determined from spline fits to external HARP pion double differential cross section data using the Sanford-Wang (SW) parametrization [32] [13]. There was no published external data for $K^{+}$production at the BNB primary proton beam energy of $8 \mathrm{GeV}$, so the Feynman scaling hypothesis was used to extrapolate $K^{+}$production measurements to this energy 15. Following a measurement by the SciBooNE collaboration [16, the uncertainty on the $K^{+}$production cross section was reduced to $14 \%$, resulting in an intrinsic $\nu_{e}$ rate (flux times cross-section) uncertainty of $18 \%$. Even with this drastic reduction, $K^{+}$production is still the largest source of uncertainty for BNB $\nu_{e}$ interactions above neutrino energies of around $0.6 \mathrm{GeV}$. With the SciBooNE result incorporated into the estimation of flux systematics, the fractional error on the $\nu_{\mu}$ flux in MicroBooNE is shown in Figure 7.4, and the fractional error on the $\nu_{e}$ flux (relevant for the low energy excess search) is shown in Figure 7.5 .

The analysis described in the following sections aims to measure the $K^{+}$production 
in the beam-line by using the rate of very high energy $\nu_{\mu}$ interactions in the MicroBooNE detector.

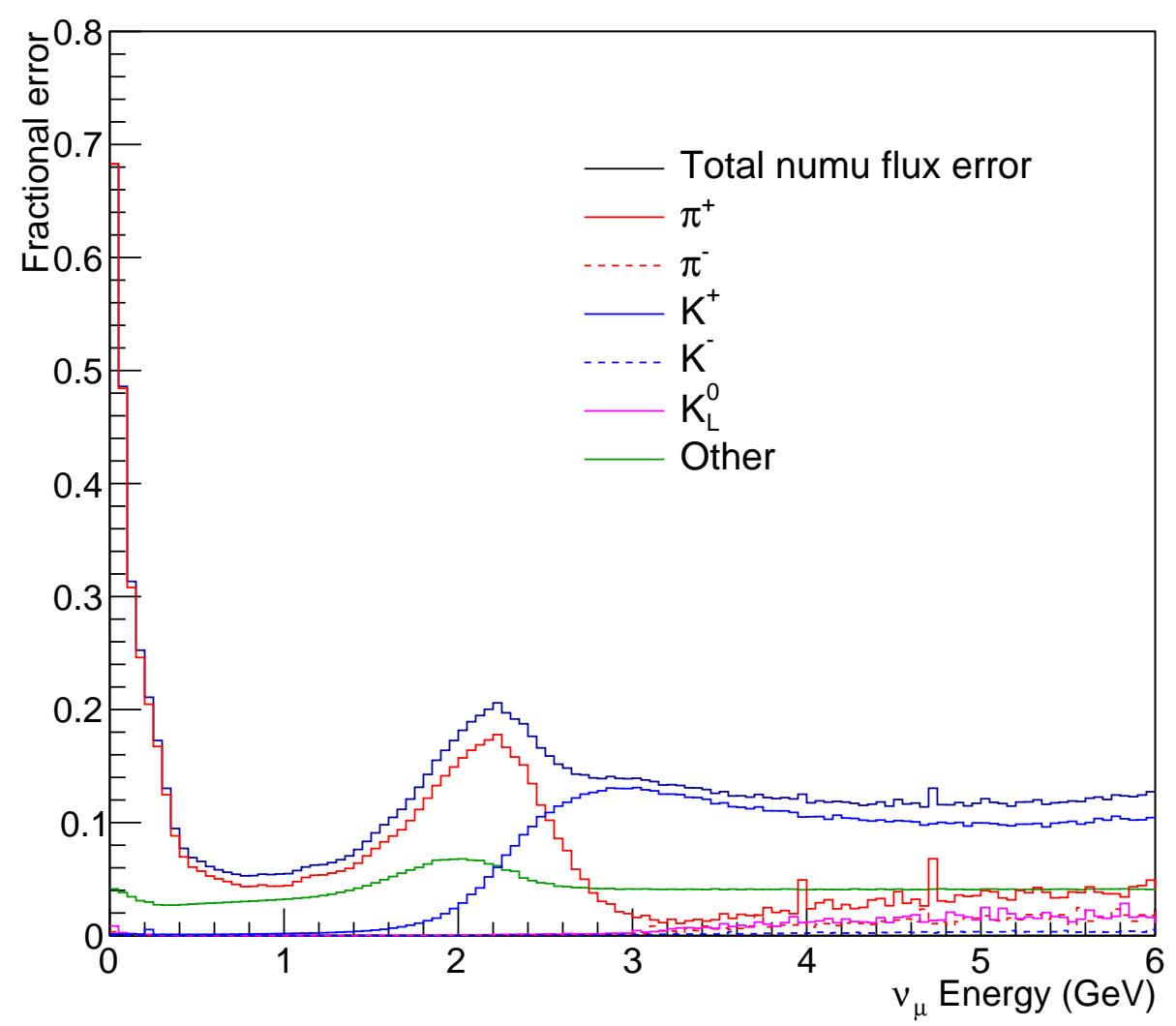

Figure 7.4: The fractional error for the BNB $\nu_{\mu}$ flux through MicroBooNE, broken up by systematic source. "Other" includes hadronic interactions, horn current uncertainty, skin depth effects, etc. The production uncertainties are divided by neutrino parent. Note the largest uncertainty at higher $\nu_{\mu}$ energies still comes from $K^{+}$production, even including the SciBooNE measurement.

\subsection{Event Selection}

The topologies of interest for this analysis are charge-current $\nu_{\mu}$ interactions in the MicroBooNE TPC. In such interactions, a $\nu_{\mu}$ interacts with a nucleon in an argon atom, 


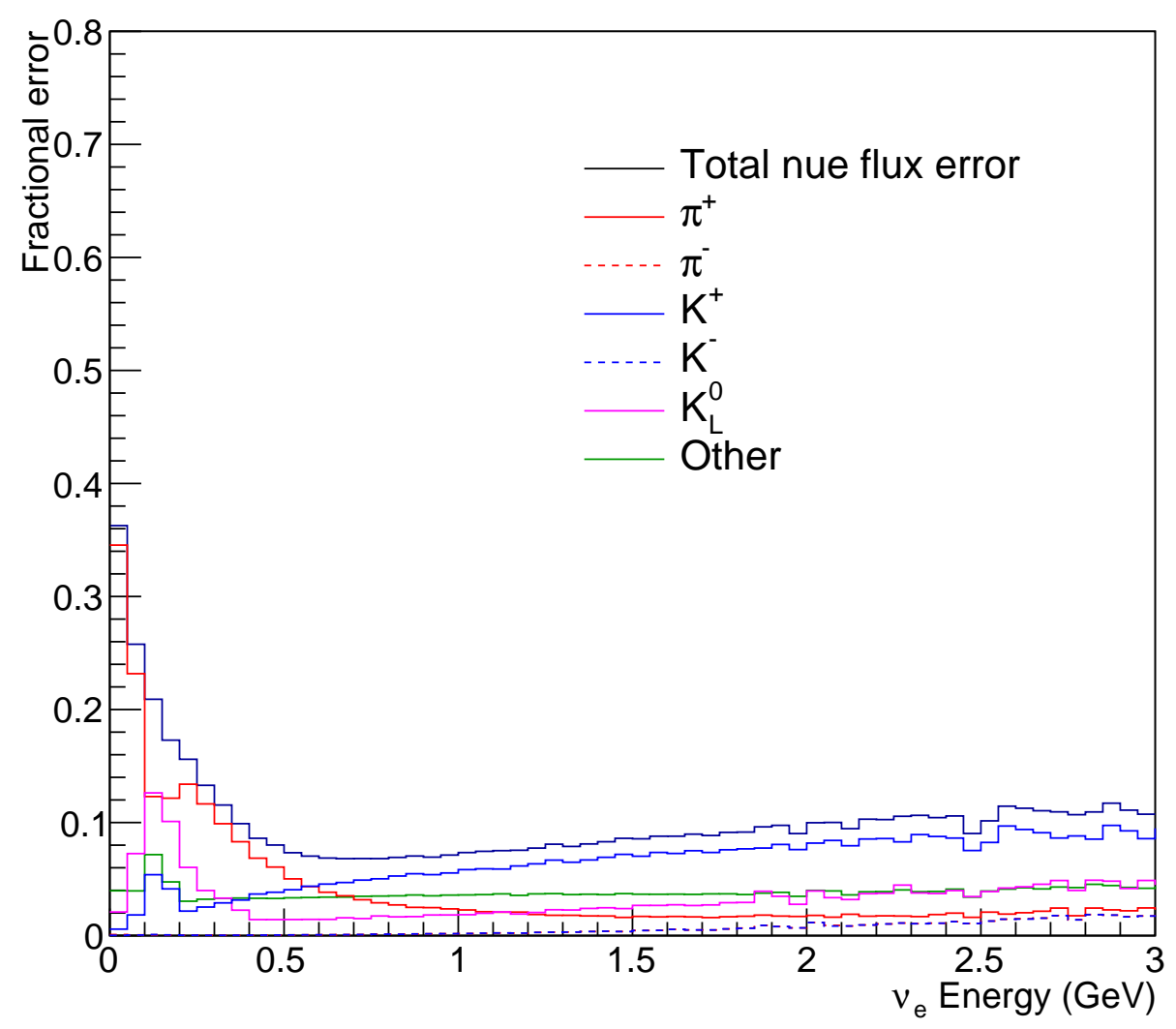

Figure 7.5: The fractional error for the BNB $\nu_{e}$ flux through MicroBooNE, broken up by systematic source. "Other" includes hadronic interactions, horn current uncertainty, skin depth effects, etc. The production uncertainties are divided by neutrino parent. Note the largest uncertainty for $\nu_{e}$ above about $0.5 \mathrm{GeV}$ neutrino energy still comes from $\mathrm{K}^{+}$production, even including the SciBooNE measurement.

exchanging a charged boson. Which particles exit the interaction depend on interaction channel and final state interactions, but in general these events have a muon in the final state. The relative probability of interaction type (quasi-elastic $Q E$, resonant production $R E S$, or deep inelastic scattering $D I S$ ) can be inferred from Figure 7.6. While most $\nu_{\mu}$ interactions in the dominant $\mathrm{BNB}$ energy region peaked at $0.7 \mathrm{GeV}$ are quasi-elastic in nature, resonant production and deep inelastic scattering become more probable at higher neutrino energies. 


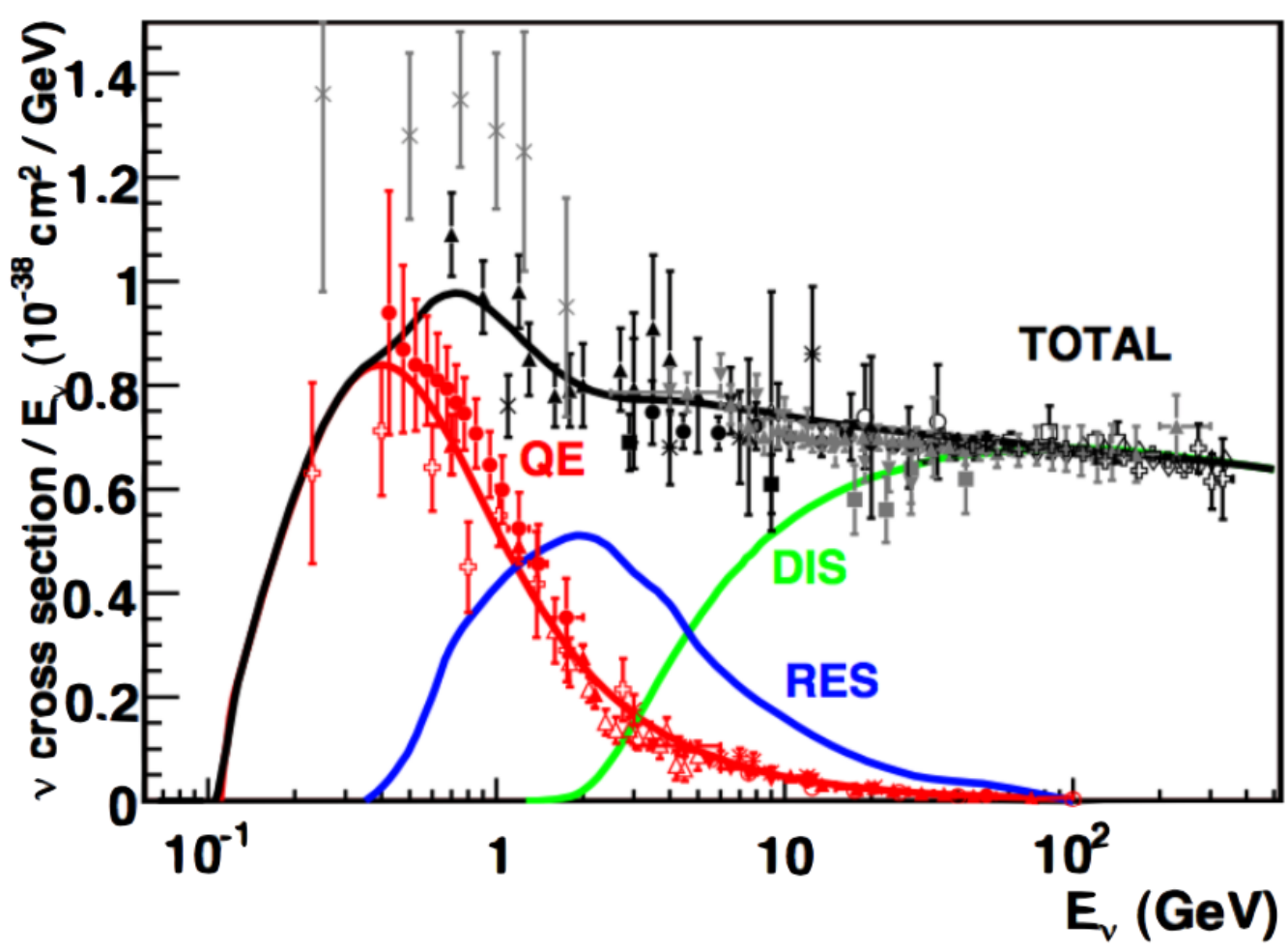

Figure 7.6: Muon neutrino charged-current cross section measurements and predictions as a function of neutrino energy. QE stands for quasielastic channels, RES stands for resonant channels, and DIS stands for deep inelastic scattering channels.

The strategy is to select $\nu_{\mu}$ interaction events in MicroBooNE, reconstruct the neutrino energy, and then place a minimum energy cut to obtain a pure sample of $\nu_{\mu}$ events originating from kaon decay in the beam-line. The interaction reconstruction, selection criteria, and neutrino energy definition are described in the following subsections.

\subsubsection{Track Reconstruction}

This section provides a brief overview of how particles traversing the detector medium are physically observed and are reconstructed in software. For a thorough description of the MicroBooNE detector, see Section 3 . 
In a $\nu_{\mu}^{C C}$ (charge current) interaction, a muon exits the interaction vertex. This particle leaves a trail of ionization electrons that create a relatively straight-line pattern in three dimensions. This line of ionization electrons is referred to as a track. Other relevant particles traversing liquid argon that create tracks are protons and charged pions. These ionization electrons are drifted by an external electric field past three anode wire planes, each with a different angle with respect to the vertical (-60 degrees, 0 degrees, and +60 degrees). There is a $3 \mathrm{~mm}$ spacing between each plane, and a $3 \mathrm{~mm}$ pitch between each wire. Scintillation light from the particle is observed by photomultiplier tubes (PMTs) situated behind the anode wire planes. The signals observed on each wire plane provides a $2 \mathrm{D}$ image of the particle track, and combining information across multiple planes allows for a complete 3D reconstruction, though there is ambiguity for the absolute location along the drift coordinate. Matching the timing of PMT signals with that of wire signals from a track clarifies this ambiguity, resulting in fully 3 -dimensional reconstructed tracks.

The pattern recognition software used in this analysis to convert detector signals to reconstructed $3 \mathrm{D}$ tracks is named Pandora [33]. This package is responsible for taking reconstructed wire signals in a triggered MicroBooNE event, clustering them on each plane (with clusters representing individual particles in the event), and matching those clusters across planes to create 3D objects. In general, event reconstruction in a LArTPC is a difficult problem which the MicroBooNE collaboration is currently working to solve. The Pandora package is a complex one which consists of a large number of nested algorithms designed to perform pattern recognition tasks, and these algorithms are not limited only to LArTPCs.

The output of the Pandora package that is relevant for this analysis are 3D reconstructed tracks created by Pandora's Projection Matching Algorithm (PMA), as well as 3D reconstructed interaction vertices which represent candidate neutrino interaction locations. Since $\nu_{\mu}^{C C}$ interactions always include a muon track exiting an interaction vertex, this is the fundamental criteria to select a sample of these CC events. More detailed criteria are needed to mitigate backgrounds, and the selection cuts are described in more detail in the 
following section.

\subsubsection{Selection Criteria}

The following selection criteria are placed on the reconstructed objects to select $\nu_{\mu}$ chargedcurrent interactions in which a candidate muon track exits the interaction vertex:

1. The event must have at least one bright (50 photoelectrons equivalent) optical flash, reconstructed from PMT timing signals, in coincidence with the expected BNB-neutrino arrival time.

2. The $z$ coordinate of the optical flash, as determined by the pulse height and timing of signals in the 32 PMTs, must be within $70 \mathrm{~cm}$ of any point on the $z$ projection of the candidate muon track.

3. Two or more reconstructed tracks must originate from the same reconstructed vertex within the fiducial volume.

4. For events with exactly two tracks originating from the vertex, additional calorimetric criteria are applied to mitigate backgrounds from cosmic muons that arrive in time with the passage of the beam, then stop and decay to an electron that is reconstructed as a track.

5. The length of the longest track associated with the interaction must be at least 15 centimeters in length.

6. For events with exactly two tracks originating from the same reconstructed vertex, they must not have exactly opposite directions (to within 5 degrees).

Selection criteria (1) and (2) are necessary to mitigate backgrounds originating from cosmic rays arriving in coincidence with the expected beam-neutrino arrival time and triggering a readout.

Selection criteria (3) is necessary to mitigate cosmic backgrounds. Cosmics entering from outside of the TPC will often lead to reconstructed neutrino vertices very close to the 
TPC boundaries. The boundaries of the fiducial volume used in this analysis are set back from the six faces of the active volume by distances of between 20 and $37 \mathrm{~cm}$, depending on the face. This volume was also chosen to reduce the impact of electric-field non-uniformities near the edges of the TPC 34, which are relevant when reconstructing a track energy (described in the next section). The fiducial volume corresponds to a mass of 55 tons.

Selection criteria (3) is also necessary to address mis-identifications stemming from track reconstruction failures. There exists a sizable background in which a cosmic traverses the entire detector, but only a portion of its track gets reconstructed, mimicking the one-track topology. These one-track events are removed at the cost of $\nu_{\mu}^{C C Q E}$ interactions in which only a muon exits the interaction vertex. Given that this analysis is geared towards the highest energy $\nu_{\mu}$ interactions which are often in the RES or DIS channel (multi-track), this cut doesn't remove many high neutrino energy signal events.

Selection criteria (4) is necessary to remove the specific topology where a cosmic ray muon stops in the detector (with an increased ionization rate as it approaches its endpoint, known as a Bragg peak) and subsequently decays into an electron. This topology has two tracks exiting a "kink", which mimics a $\nu_{\mu}^{C C}$ topology. This cut leverages the presence of the Bragg peak to correctly identify the directions of the two tracks and therefore remove this event from the analysis.

Selection criteria (5) and (6) are additional cuts to mitigate specific track reconstruction failure modes that are present when reconstructing cosmic rays. In these failure modes, a long straight track can be broken up and reconstructed as two or more straight tracks, with a reconstructed vertex as the breaking point.

The above selection criteria serve only to select a sample of $\nu_{\mu}^{C C}$ events, which come from both pion and kaon decay. The efficiency to select these events these is defined as

$$
\epsilon_{\nu_{\mu}^{C C}}=\frac{\# \text { selected events }}{\# \text { true } \nu_{\mu}^{C C} \text { events in the fiducial volume }}
$$


and the purity is defined as

$$
\pi_{\nu_{\mu}^{C C}}=\frac{\# \text { true } \nu_{\mu}^{C C} \text { correctly identified events in selected sample }}{\# \text { selected events }} .
$$

With the described selection criteria, the efficiency $\epsilon_{\nu_{\mu}^{C C}}$ is $27 \%$ in simulation. This efficiency is rather low because of the requirement of two reconstructed tracks exiting the interaction combined with a low track reconstruction efficiency, especially for short tracks near the vertex 1 . The purity is $82 \%$, and the primary contaminating backgrounds are those from cosmics. The backgrounds to this analysis are discussed in more detail in the following section.

\subsubsection{Backgrounds}

There are three main backgrounds to this $\nu_{\mu}^{C C}$ from $K^{+}$decay search: $\nu_{\mu}^{C C}$ from $\pi$ decay interactions, neutral current interactions, and cosmic-induced backgrounds. The $\nu_{\mu}^{C C}$ from $\pi$ decay interactions are the most dominant background and will be removed with a cut on neutrino energy, which is described in the following section.

The neutral current backgrounds occur when a beam neutrino of any flavor interacts in such a way as to mimic a $\nu_{\mu}^{C C}$ interaction. For example, a neutrino can interact with a nucleus and liberate a proton and a charged pion. In this case, the charged pion may be mis-identified as a muon.

The cosmic-induced backgrounds are mitigated largely by the previously described event selection cuts. Their topologies are usually caused either by track reconstruction failures, or by stopping muons which decay into electrons. In the latter example, the track direction of the muon must be incorrect, and the decay electron must be reconstructed as a separate track. Though many calorimetric and track-length based selection criteria are used to reduce this background, it still persists because the rate of stopping cosmics in triggered

\footnotetext{
${ }^{1}$ For this track reconstruction algorithm, the efficiency to reconstruct the muon in a $\nu_{\mu}^{C C}$ interaction is on the order of $80 \%$ for muons with momenta above $0.4 \mathrm{GeV}$, sharply decreasing to $30 \%$ for muons with momenta around $0.1 \mathrm{GeV}$.
} 
readouts far outweighs that of $\nu_{\mu}^{C C}$ interactions.

With the events selected, the next step is to reconstruct the neutrino energy. This is necessary because the neutrino energy is the variable through which the $\nu_{\mu}$ from $K^{+}$decay sample of interest is isolated.

\subsection{Neutrino Energy Reconstruction}

Event selection (Section 7.2.2 provides a sample of candidate $\nu_{\mu}^{C C}$ interactions consisting of a number $(>2)$ of reconstructed tracks exiting a common neutrino interaction vertex. Each track must first be associated with a particle identity (referred to as PID) in order to compute the energy of that track. Note that in $\nu_{\mu}^{C C} \pi^{0}$ interactions there will also be two showers exiting the vertex from the neutral pion decay. However, these showers (and therefore their associated energy) are ignored. The reason for this is that at the time of this analysis, the shower reconstruction performance in MicroBooNE software isn't at an adequate level to include them. Excluding them will ultimately worsen the energy reconstruction performance, but the performance is still sufficient to select a pure sample of $\nu_{\mu}^{C C}$ interactions from $K^{+}$decay, as will be shown later.

The longest track associated with the interaction is assumed to be the muon, which is reasonable because on average around half of the neutrino energy in a $\nu_{\mu}^{C C}$ interaction is transferred to the outgoing muon, as shown in Figure 7.7. Also, muons are in general closer to minimally ionizing than to other final state particles like protons and therefore produce longer tracks.

The remaining tracks exiting the interaction are classified as either charged pions, or protons. This is reasonable because only one muon can exit a $\nu_{\mu}^{C C}$ interaction vertex (barring negligibly rare topologies in which a charged pion is created and decays within the nucleus, resulting in two muons in the final state). Protons are much more highly ionizing than charged pions, and therefore the measured $\mathrm{dE} / \mathrm{dx}$ of the tracks are used in classifica- 


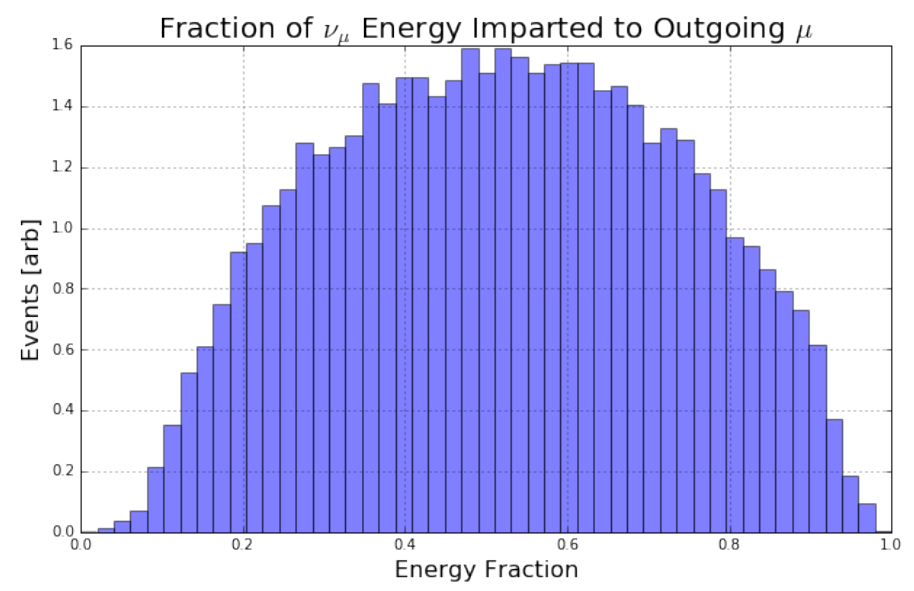

Figure 7.7: The fraction of $\nu_{\mu}$ energy imparted to the outgoing lepton $(\mu)$ in $\nu_{\mu}^{C C}$ interactions.

tion; shorter, highly ionizing tracks are tagged as protons, and longer, lower ionizing tracks are tagged as charged pions.

The reconstructed neutrino energy is defined as the sum of the energies of all tracks exiting the interaction vertex. For muon and pion tracks, their total energies are added (including mass), while for proton tracks only the kinetic energy is added. This is because while the muons and pions are created with energy directly from the neutrino, the neutrino is scattering off of a nucleon ( $n$ or $p$ ) and is producing a nucleon ( $p$ or $n)$. Since neutrons and protons have very similar masses, one doesn't need to include the outgoing nucleon mass in the energy calculations. This simplistic energy definition also neglects binding energy in the nucleus, but that effect is small.

The method used to reconstruct the energy of the muon track depends on whether that track is fully contained in the fiducial volume. In general, reconstructing the energies of fully contained particle tracks in a LArTPC is straightforward, either with a range-based approach, or a calorimetric approach (since calorimetric information can be gleaned from the size of sense-wire signals). In this analysis, range-based energy is used for fully contained muons. The stopping power of muons in liquid argon is well described by the continuous 
slowing-down approximation (CSDA) by the particle data group, and agrees with data at the sub-percent level 35 [36]. By using a linear interpolation between points in the stopping power table of ref. [36], the length of a track can be used to reconstruct the muon's total energy with resolution better than $4 \%$ and negligible bias.

For muon tracks that exit the fiducial volume (which is ultimately the case for all selected $\nu_{\mu}^{C C}$ from $K^{+}$decay), a different method is required to compute the track energy. This method leverages a phenomenon called multiple Coulomb scattering (MCS), and the development and characterization of this method is the subject of the next chapter and a pending publication by the author of this thesis. The MCS energy resolution for well reconstructed exiting muon tracks with at least one meter of their length contained in the fiducial volume is energy dependent and on the order of $15 \%$ for muons with momenta below $2.5 \mathrm{GeV} / \mathrm{c}$, and on the order of $30 \%$ for muons with momenta between 2.5 and 4.0 $\mathrm{GeV} / \mathrm{c}$. A downside to the MCS method is that it requires at least a meter of track to be contained. For muons that exit with less than a meter contained, the range-based energy is necessarily used, which is often a significant underestimation of the true energy. Note that, at this point, space charge effects most predominantly located near the edges of the TPC are not included in this simulation. These electric-field non-uniformities have the effect of bending a track, which will cause the MCS technique to underestimate the track's energy. To remove the difference between data and Monte-Carlo, all reconstructed tracks in this analysis are truncated to be contained within the fiducial volume, the dimensions of which were chosen to include a region in which the effect of electric field non-uniformities are small. This effectively converts the fiducial volume to an active volume, in which space charge effects are negligible.

Charged pion tracks are treated in much the same way as muon tracks. When they are contained, range-based energy is used, and when they exit, MCS energy is used (despite the fact that MCS energy is tuned for muons, the same method works sufficiently well for the purposes of this analysis on pions). Proton tracks are in general much shorter and therefore their probability of exiting the fiducial volume is small. For these tracks, a range-based 
energy is used based on the stopping power of protons in liquid argon published by the same aforementioned references.

Given the described event selection criteria, particle identification techniques, and track energy methods, a distribution of the reconstructed neutrino energy versus true neutrino energy for a sample of correctly identified $\nu_{\mu}^{C C}$ interactions in MicroBooNE simulation is shown in Figure 7.8. It can be seen that the reconstructed energy tends to be an underestimation of the true energy. This is caused by the failure to include shower-based energy from the interaction (as mentioned previously), as well as needing to use range-based energy for tracks which have less than one meter contained in the TPC. While this reconstructed neutrino energy could be improved upon, its performance is sufficient for this analysis. As you can see from the figure, placing a cut on reconstructed neutrino energy at around 2.5 $\mathrm{GeV}$ will provide a relatively pure sample of events in which the true neutrino energy is also above $2.5 \mathrm{GeV}$.

\subsection{Results in Simulation}

The simulation used for this analysis consists of BNB simulated neutrino interactions within the entire MicroBooNE TPC. The BNB flux is described in Chapter 4 of this thesis. The neutrino interaction event generator used is GENIE [25]. These interactions also include simulated cosmic rays by the CORSIKA package 27. This "BNB + Cosmic" simulation provides the measurement sample as well as all relevant backgrounds. If a reconstructed neutrino vertex is within $3 \mathrm{~cm}$ of a true $\nu_{\mu}^{C C}$ interaction vertex, that event will be classified as either $\nu_{\mu}$ from pion decay (background) or $\nu_{\mu}$ from kaon decay (signal), depending on the true neutrino parent. If the reconstructed neutrino vertex is within $3 \mathrm{~cm}$ of a true $\nu_{x}^{N C}$ interaction vertex, that event is classified as a neutral current background. Lastly, if the reconstructed neutrino vertex is not near any true neutrino interaction point, the interaction is classified as cosmic.

$2.2 \times 10^{20}$ protons-on-target are simulated in this analysis, and the final histograms are 


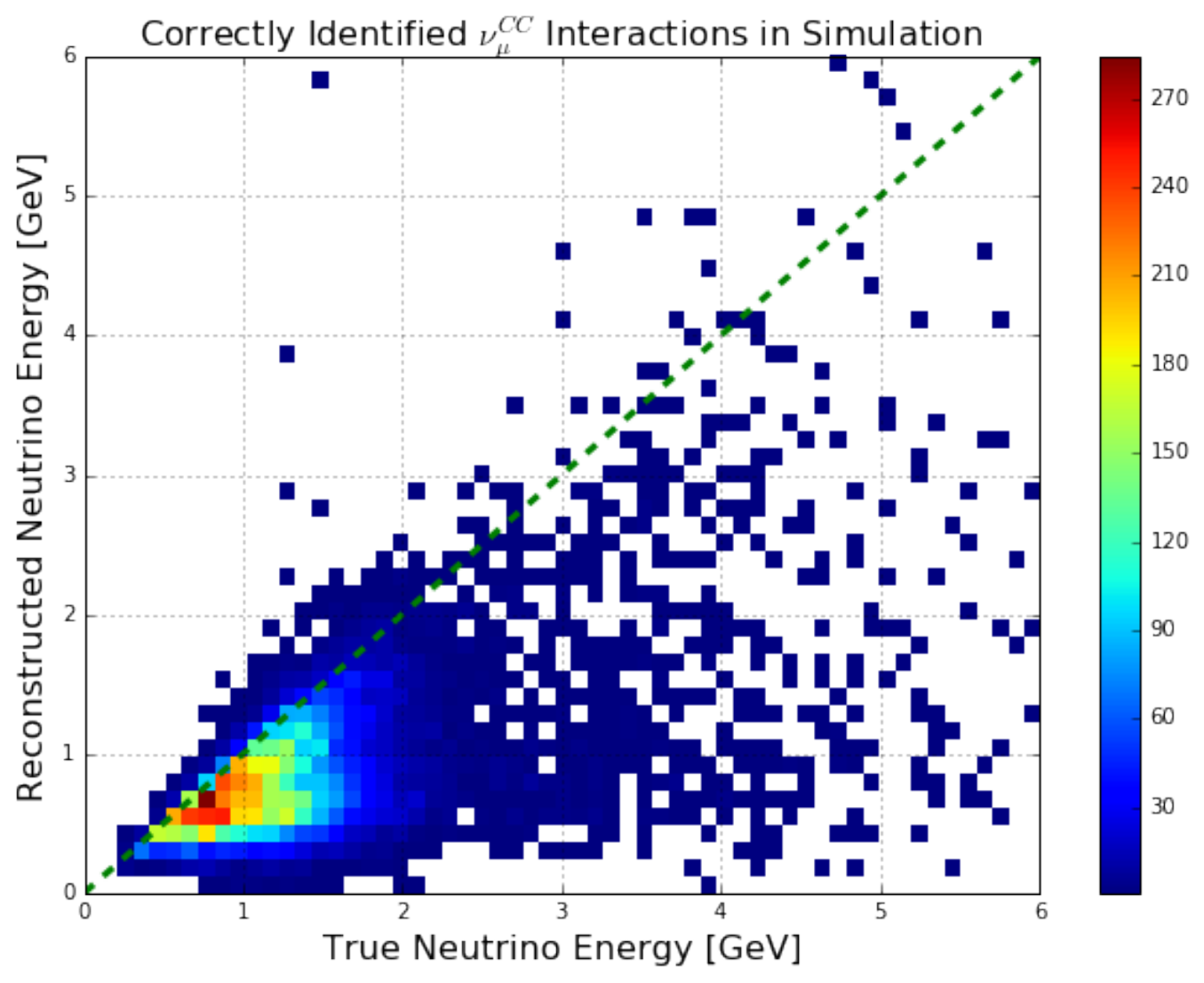

Figure 7.8: Reconstructed neutrino energy versus true neutrino energy for a sample of correctly identified BNB $\nu_{\mu}^{C C}$ interactions in the MicroBooNE TPC.

renormalized to $0.5 \times 10^{20}$. This scaling is done because when a comparison to data is made (in the next section), only $0.5 \times 10^{20}$ protons-on-target worth of data are available for this analysis due to the blinding requirements imposed within the MicroBooNE collaboration. Note that the nominal amount of protons-on-target scheduled to be delivered to MicroBooNE over a three-year running period is much more than this, $6.6 \times 10^{20}$; unblinding the full data set in the future will improve the strength of this analysis' result by decreasing the statistical uncertainty. At the time this thesis was written, MicroBooNE has accrued roughly $80 \%$ of the expected protons-on-target, corresponding to $5.3 \times 10^{20}$.

The distribution of simulated signal and backgrounds as a function of reconstructed neutrino energy between 0 and $2.5 \mathrm{GeV}$ (which is referred to as the sideband region for this 
analysis, and contains $98.8 \%$ of all selected events are contained in this region, though very few neutrinos from kaon decay) in Figure 7.9. In this stacked histogram figure, the green histogram represents $\nu_{\mu}^{C C}$ interactions from kaon decay, which is the signal of interest. In this sideband region the $\nu_{\mu}^{C C}$ from pion decay (blue) is the dominant background, while cosmic-induced backgrounds (red) are mostly relevant at reconstructed neutrino energies below $1 \mathrm{GeV}$. Neutral current backgrounds (yellow) are sub-dominant.

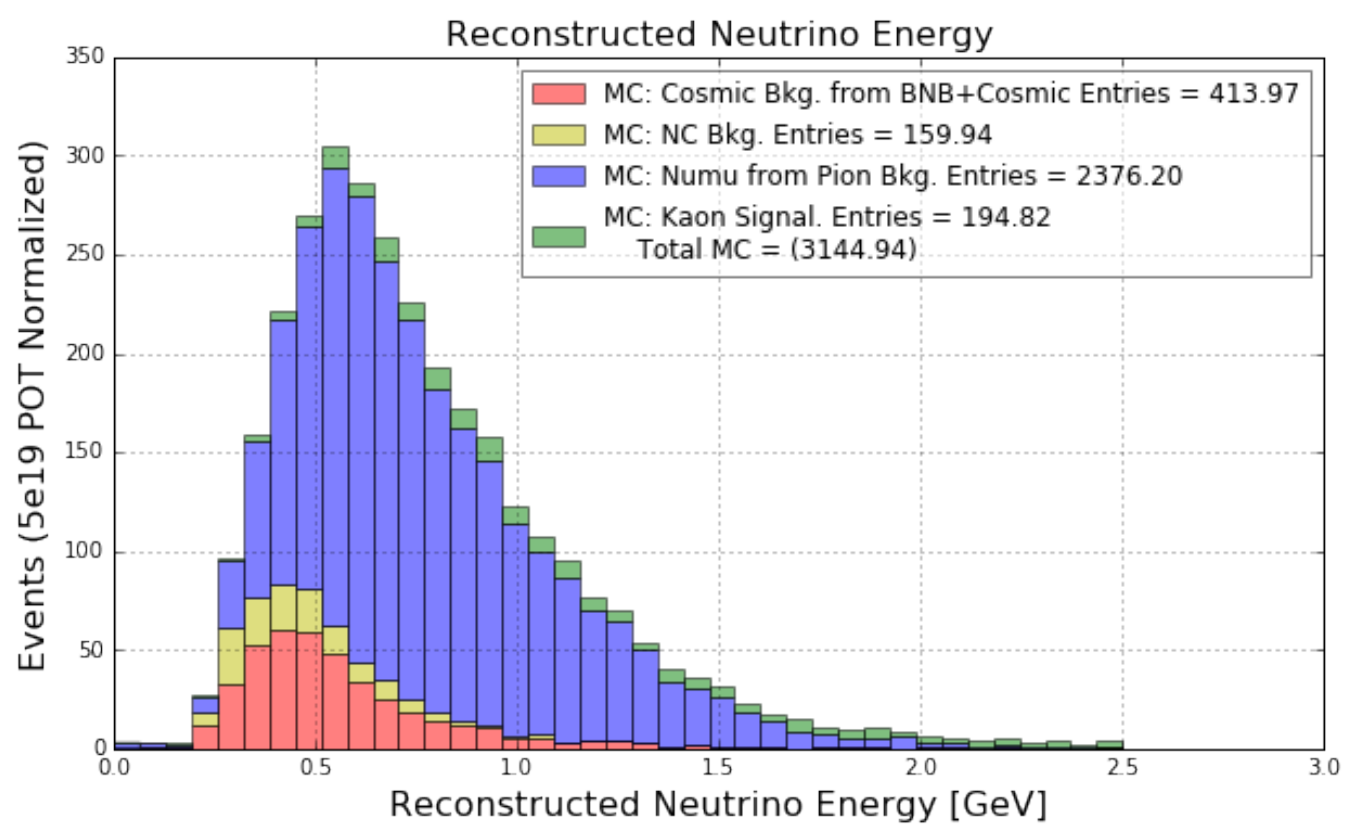

Figure 7.9: Distribution of signal (green) and backgrounds normalized for $0.5 \times 10^{20}$ protonson-target worth of data, for reconstructed neutrino energy below $2.5 \mathrm{GeV}$. This comprises the sideband region.

In order to select a relatively pure sample of $\nu_{\mu}^{C C}$ from $K^{+}$decay interactions, a cut on reconstructed neutrino energy is placed at $2.5 \mathrm{GeV}$. The resulting sample has a kaon signal purity of $81 \%$, and is shown in Figure 7.10 . In this region, the backgrounds from neutral current interactions and from $\nu_{\mu}^{C C}$ from $\pi$ decay are drastically suppressed. Still remaining is a cosmic-induced background which comprises $15 \%$ of the sample. The correlation between energy and angle of all kaons which decay to produce $\nu_{\mu}^{C C}$ interactions in the fiducial volume is shown in Figure 7.11, and the subset of those which are reconstructed and selected in this 
analysis to pass the $2.5 \mathrm{GeV}$ cut on neutrino energy are shown in Figure 7.12 . The projection of these distributions onto the angle axis are shown in Figure 7.13, and onto the energy axis in Figure 7.14. The mean energy and angle information for kaons which produce $\nu_{\mu}$ (all, and selected) and for those which produce $\nu_{e}$ (relevant for the electron-like low energy excess analysis) are summarized in Table 7.1. The kaons selected in the signal region tend to be skewed towards being more forward-going (smaller angle) and having higher energy, though the production phase-space is still reasonably covered by the selection (as seen by comparing Figures 7.11 and 7.12 .

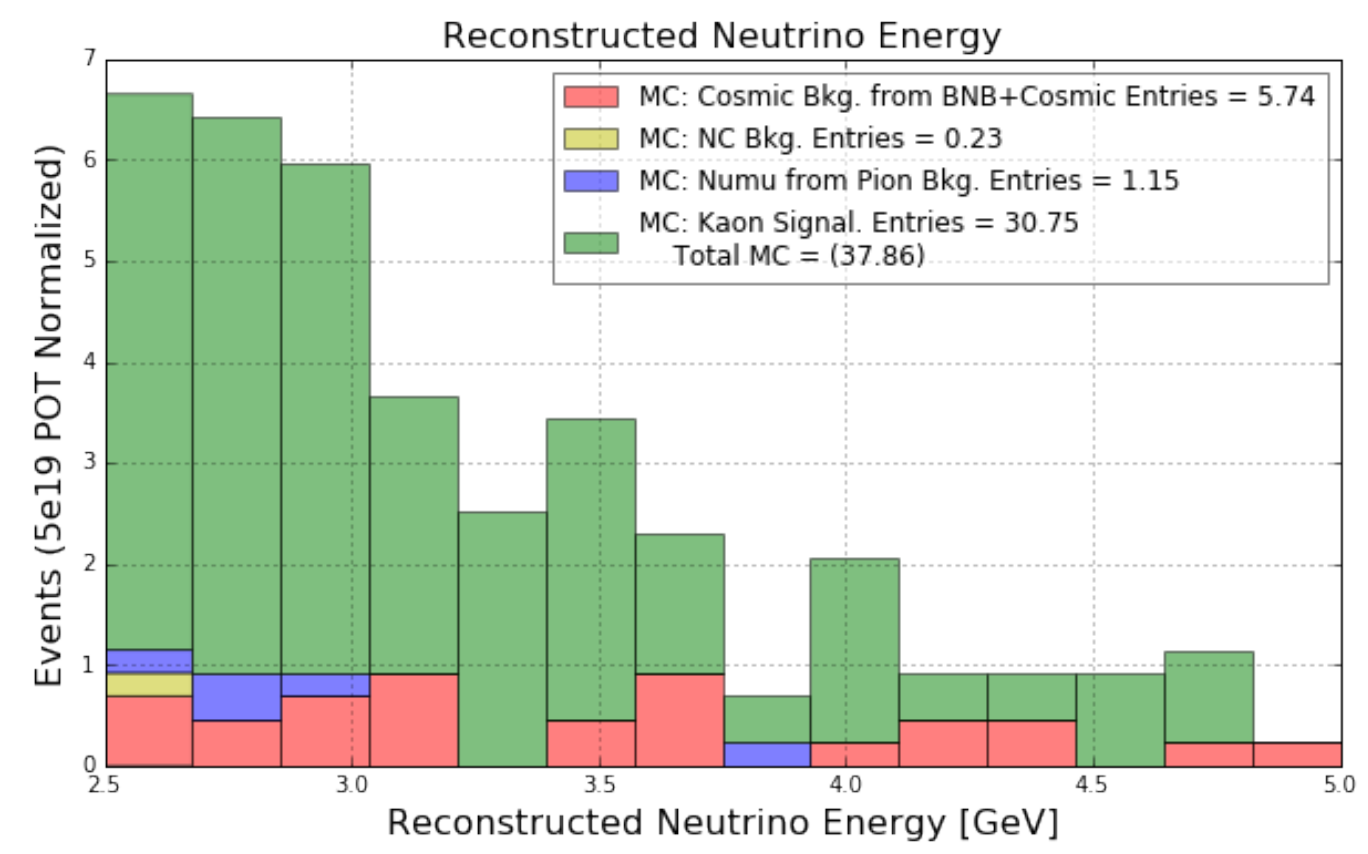

Figure 7.10: Distribution of signal (green) and backgrounds normalized for $0.5 \times 10^{20}$ protons-on-target worth of data, for reconstructed neutrino energy between 2.5 GeV and $5 \mathrm{GeV}$. This comprises the signal region, which has an $81 \%$ purity of $\nu_{\mu}^{C C}$ interactions from $K^{+}$decay in the beam.

\subsection{Sideband Data and Simulation Comparisons}

As shown in the previous section, the event reconstruction and selection is able to provide a relatively pure signal sample of $\nu_{\mu}^{C C}$ from kaon decay interactions in the detector by choos- 


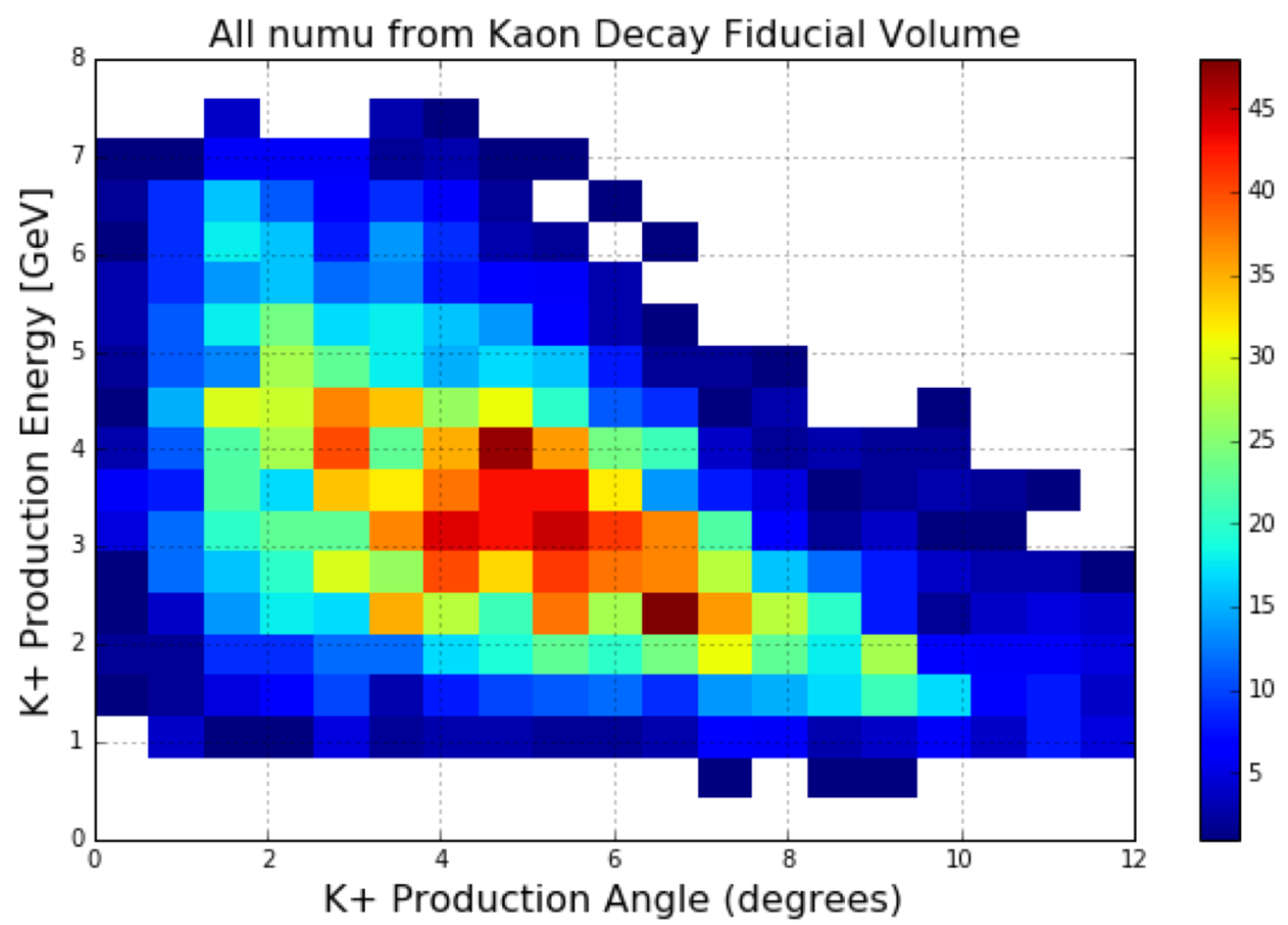

Figure 7.11: A two-dimensional plot of energy versus angle for all kaons in the beam producing $\nu_{\mu}^{C C}$ interactions in the detector.

ing those with reconstructed neutrino energy above $2.5 \mathrm{GeV}$. While the sideband region (with reconstructed neutrino energy below $2.5 \mathrm{GeV}$ ) is composed primarily of backgrounds from $\nu_{\mu}^{C C}$ from $\pi$ decay interactions, it is used for lower-level comparisons between data and simulation to increase confidence in results from the signal region. This section will show several such comparison plots.

In order to compare data to simulation, there is a subtlety involving the cosmic background which needs to be taken into account. In the simulation, each triggered readout event has a neutrino interacting somewhere within the TPC, along with several simulated cosmic rays. However, in real data, the majority of triggers are induced by cosmic interactions arriving during the expected neutrino arrival timing window $(1.6 \mu s)$, and do not contain a neutrino interaction at all. To account for this, a sample of external (EXT) triggered data is taken when the neutrino beam is turned off, in order to get an estimate of the 


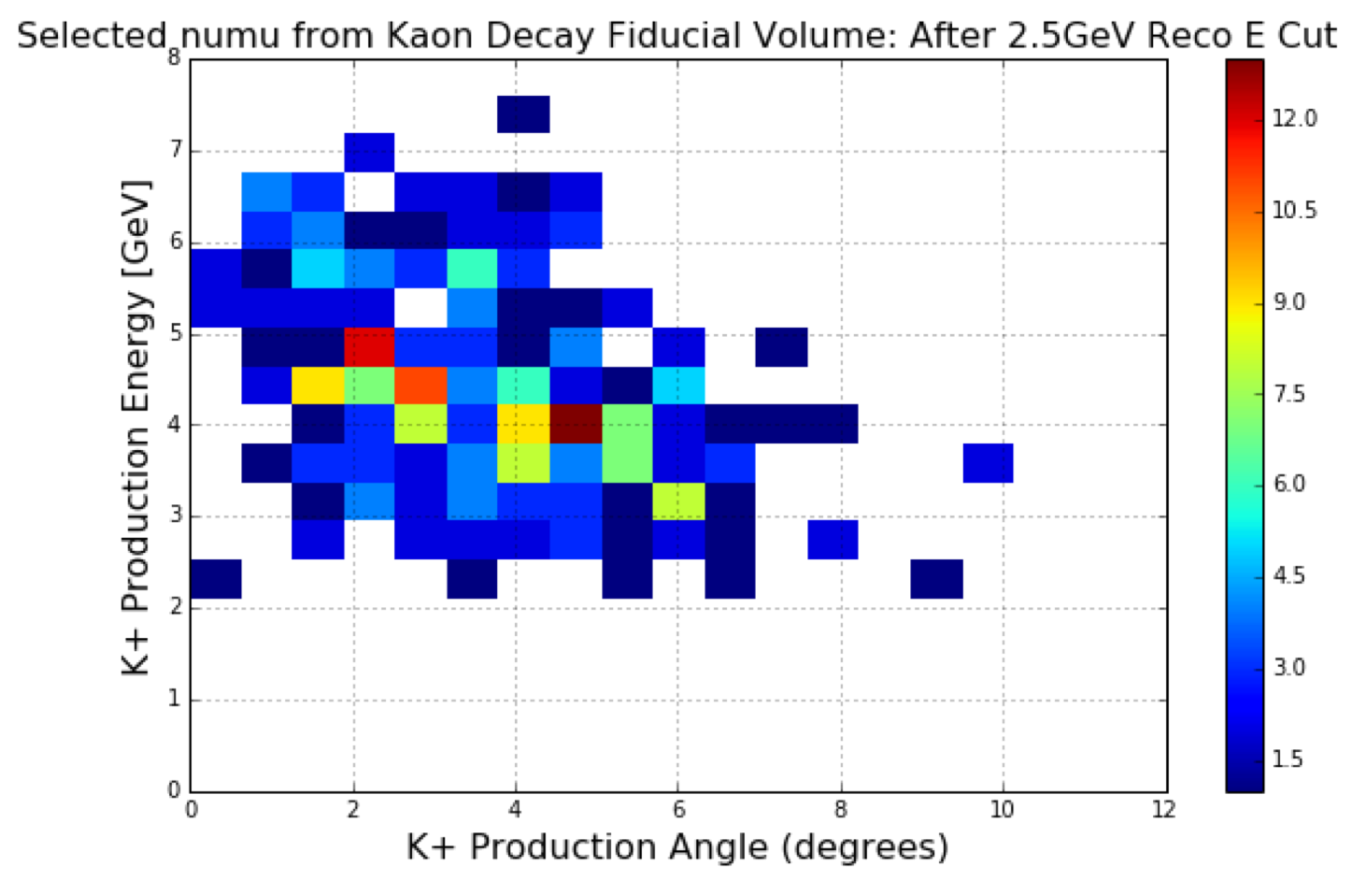

Figure 7.12: A two-dimensional plot of energy versus angle for the subset of kaons in Figure 7.11 which are reconstructed and selected for this analysis (having reconstructed neutrino energy above $2.5 \mathrm{GeV}$ ).

cosmic background. This "off-beam" data is then subtracted from the "on-beam" data in the analysis, and the difference is what is directly comparable to simulation. The normalization factor between "off-beam" and "on-beam" data is computed based on the number of measured bright reconstructed optical flashes within the expected neutrino arrival window both during "off-beam" (cosmic-only) runs and "on-beam" runs. This factor is computed to be 0.844 , which means that $84 \%$ of triggered BNB readouts relevant to this analysis in MicroBooNE data are cosmic-induced. Note that while subtracting "off-beam" data from "on-beam" data accounts for the cosmic backgrounds not included in simulation, there still exists a cosmic background originating from readouts which are truly triggered by a neutrino interaction, but with a cosmic arriving during the milliseconds-long readout window that is mistakenly identified as the selected neutrino interaction. This is the background shown in red in the previously shown stacked histograms (for example in Figure 7.9). In the forthcoming data-MC comparison plots, the "off-beam" data points are shown in cyan 


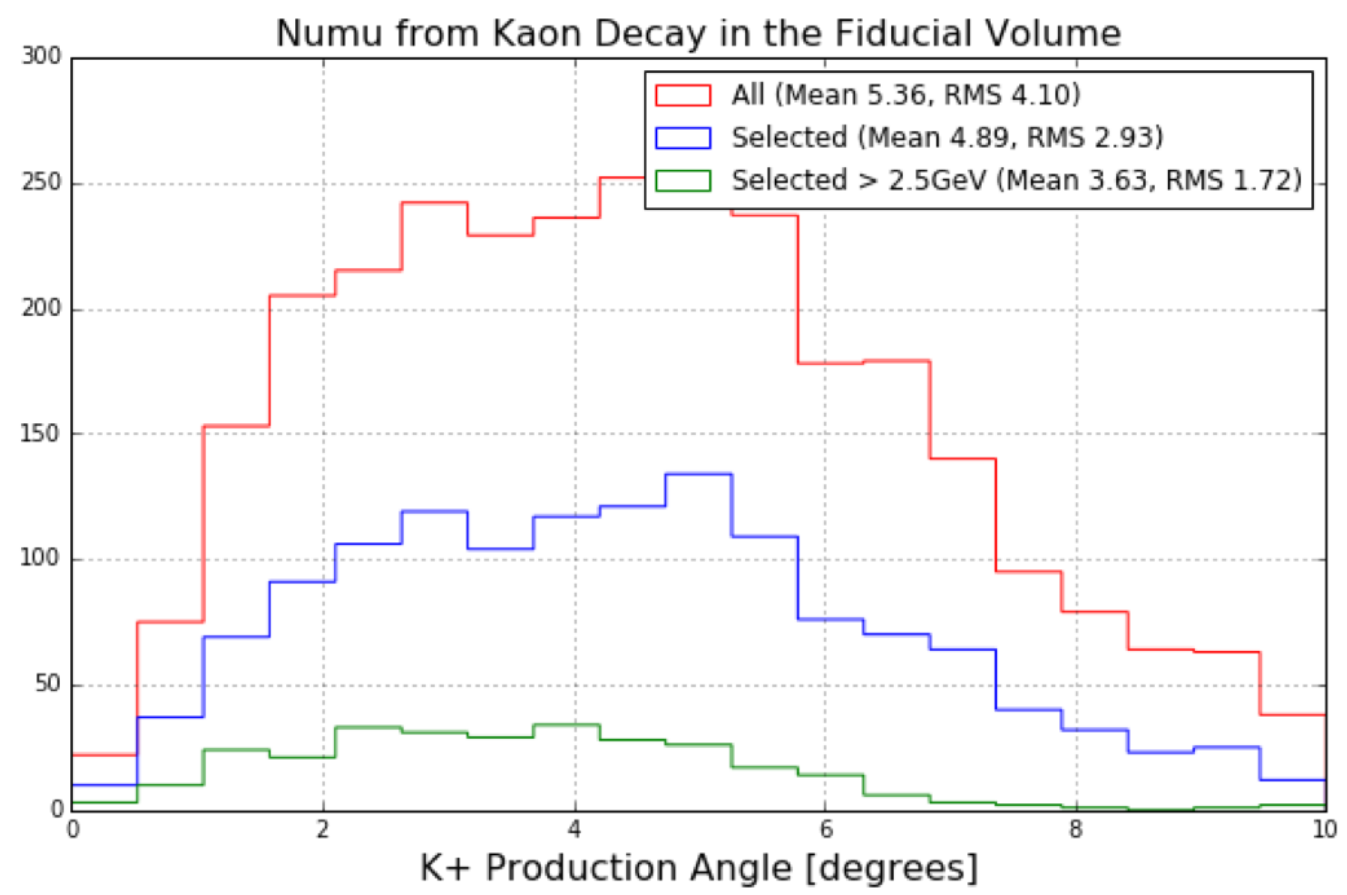

Figure 7.13: The kaon production angle distribution for all kaons in the beam producing $\nu_{\mu}^{C C}$ interactions in the detector (red), the subset of those which are reconstructed and selected in both the sideband and signal region (blue) and the subset of those in the Kaon enriched signal sample, with reconstructed neutrino energy above 2.5 GeV (green).

for reference only; the purple points are the result of their subtraction from the on-beam data.

The first low level data to simulation comparison done in the sideband region is a distribution of the 3D length of the muon track associated with the interaction, and is shown in Figure 7.15. This plot shows the simulated background and signals as a stacked histogram with the same color-coding as shown previously (Figure 7.9. . Statistical error bars are drawn on the purple data points, taking into account the statistical uncertainties associated with the subtraction of the "on-beam" and "off-beam" samples. Below the main figure is a bin-by-bin ratio plot of data divided by simulation. We see a normalization difference over the entire length range of 0 to $600 \mathrm{~cm}$ between data and simulation of about $8 \%$, with data having fewer events than expected in simulation. This normalization difference is not 


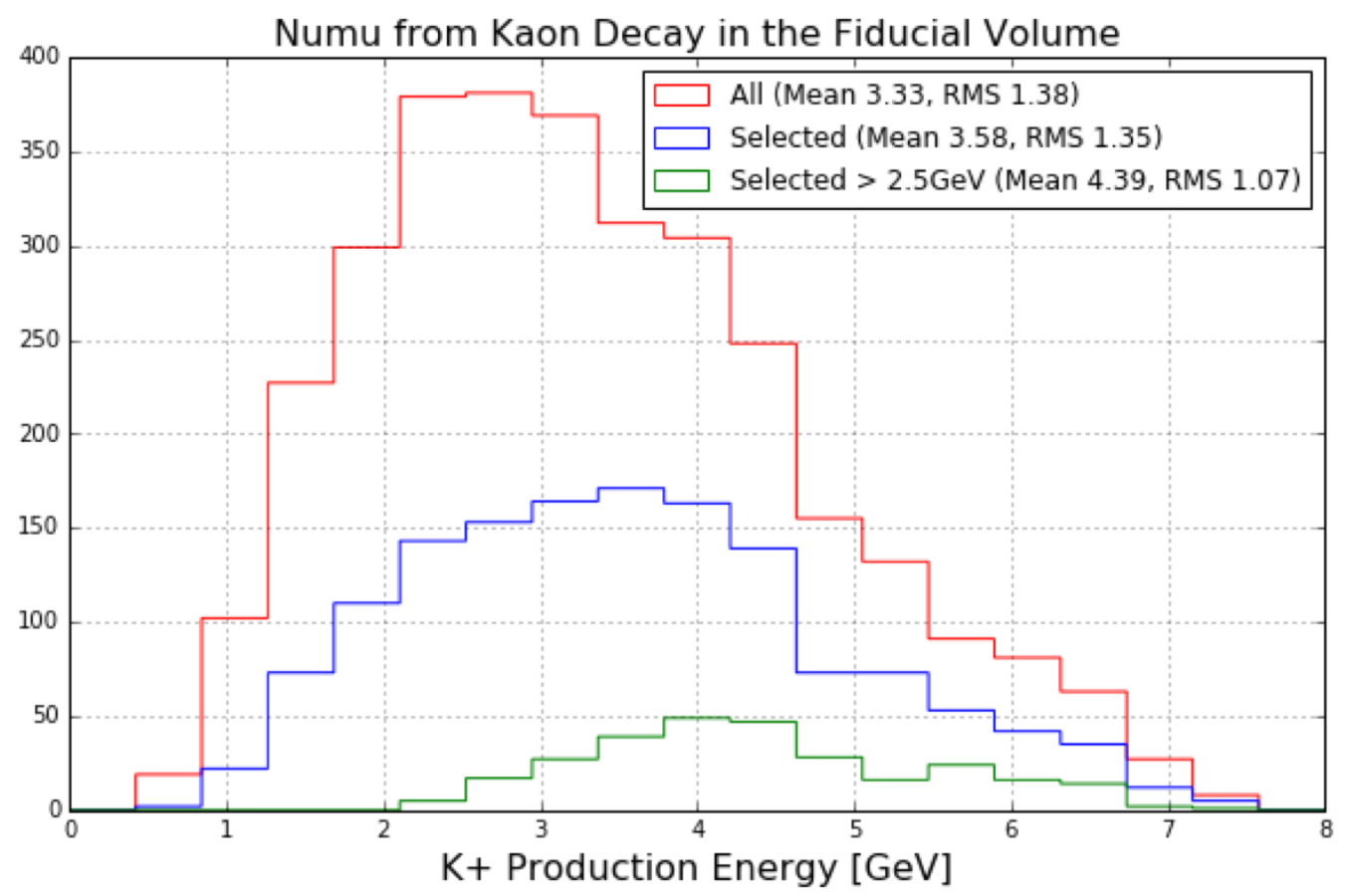

Figure 7.14: The kaon production energy distribution for all kaons in the beam producing $\nu_{\mu}^{C C}$ interactions in the detector (red), the subset of those which are reconstructed and selected in both the sideband and signal region (blue) and the subset of those in the Kaon enriched signal sample, with reconstructed neutrino energy above 2.5 GeV (green).

flat with length; the data deficit is more prominent for longer length tracks, perhaps attributable to noise sources in the detector not adequately modeled in simulation. Such noise sources include broken or sporadically noisy anode plane sense wires that tend to prevent the longest tracks from being fully reconstructed. The overall normalization difference in the sideband serves as a calibration factor to be applied to the signal region.

The next low-level data to simulation comparison in the sideband region is shown in Figure 7.16. This is the track multiplicity (the number of reconstructed tracks associated with the interaction). The same normalization offset of $8 \%$ persists, as these are the same events as in Figure 7.15. While data and simulation agree for two- and three-track events, they begin to disagree for other multiplicities. The reason for this could be imperfect modeling of nuclear interactions (including final-state intra-nuclear interactions) within the neutrino 


\begin{tabular}{|l|l|l|l|l|}
\hline \multicolumn{5}{|c|}{ Kaon Production Summary } \\
\hline & All $\overline{K_{\theta}}[\mathrm{deg}]$ & Selected $\overline{K_{\theta}}[\mathrm{deg}]$ & All $\overline{K_{E}}[\mathrm{GeV}]$ & Selected $\overline{K_{E}}[\mathrm{GeV}]$ \\
\hline \hline$\nu_{\mu}$ & $5.36[4.10]$ & $3.63[1.72]$ & $3.33[1.38]$ & $4.39[1.07]$ \\
\hline$\nu_{e}$ & $5.10[3.68]$ & $\mathrm{N} / \mathrm{A}$ & $3.32[1.28]$ & $\mathrm{N} / \mathrm{A}$ \\
\hline \hline
\end{tabular}

Table 7.1: A summary of the mean kaon production angle $\left(\overline{K_{\theta}}\right)$ and energy $\left(\overline{K_{E}}\right)$ in the $B N B$ for $\nu_{\mu}$ interactions (all interactions interacting within the TPC, and the subset of those which are selected in the analysis), and for $\nu_{e}$ interactions (providing backgrounds to the electron-like low energy excess search). Reported in brackets is the RMS of each distribution.

interaction simulation package.

The next two data to simulation comparisons in the sideband region are shown in Figures 7.17 and 7.18 . In these plots, the difference between data and simulation becomes sizable. The first plot is the $\phi$ angle of the muon track associated with the interaction, where $\phi$ is measured in radians with respect to the vertical, around the beam direction. Naively the shape of this distribution should be flat, but the dips at $\pm \frac{\pi}{2}$ correspond to tracks oriented along the drift direction, which are difficult to reconstruct because of the geometry of the sense wires. These dips are well matched between data and simulation but there are big discrepancies at 0 and $\pm \pi$ radians which are not understood. The second plot is the $\theta$ angle of the muon track, measured with respect to the beam direction. A clear deficit of forward-going tracks (with small $\theta$ ) can be seen in data, with an excess of vertically-oriented tracks (with $\theta \approx \frac{\pi}{2}$ ). This difference between data and simulation is particularly important because the high energy $\nu_{\mu}^{C C}$ interactions which comprise the kaon-enriched signal region have generally very forward-going muons. This difference might be attributed to poor neutrino interaction kinematics by GENIE in which GENIE incorrectly models the outgoing muon angles, though there are no other analyses yet which confirm this. Additionally, the difference might be attributed to a mis-estimation of the cosmic background, since cosmic backgrounds tend to be more vertical with $\theta$ above 1 radian, where the data excess occurs. Since the off-beam subtraction technique appropriately handles all out-of-time cosmic backgrounds, this mis-estimation would apply only to events in which a neutrino triggered 


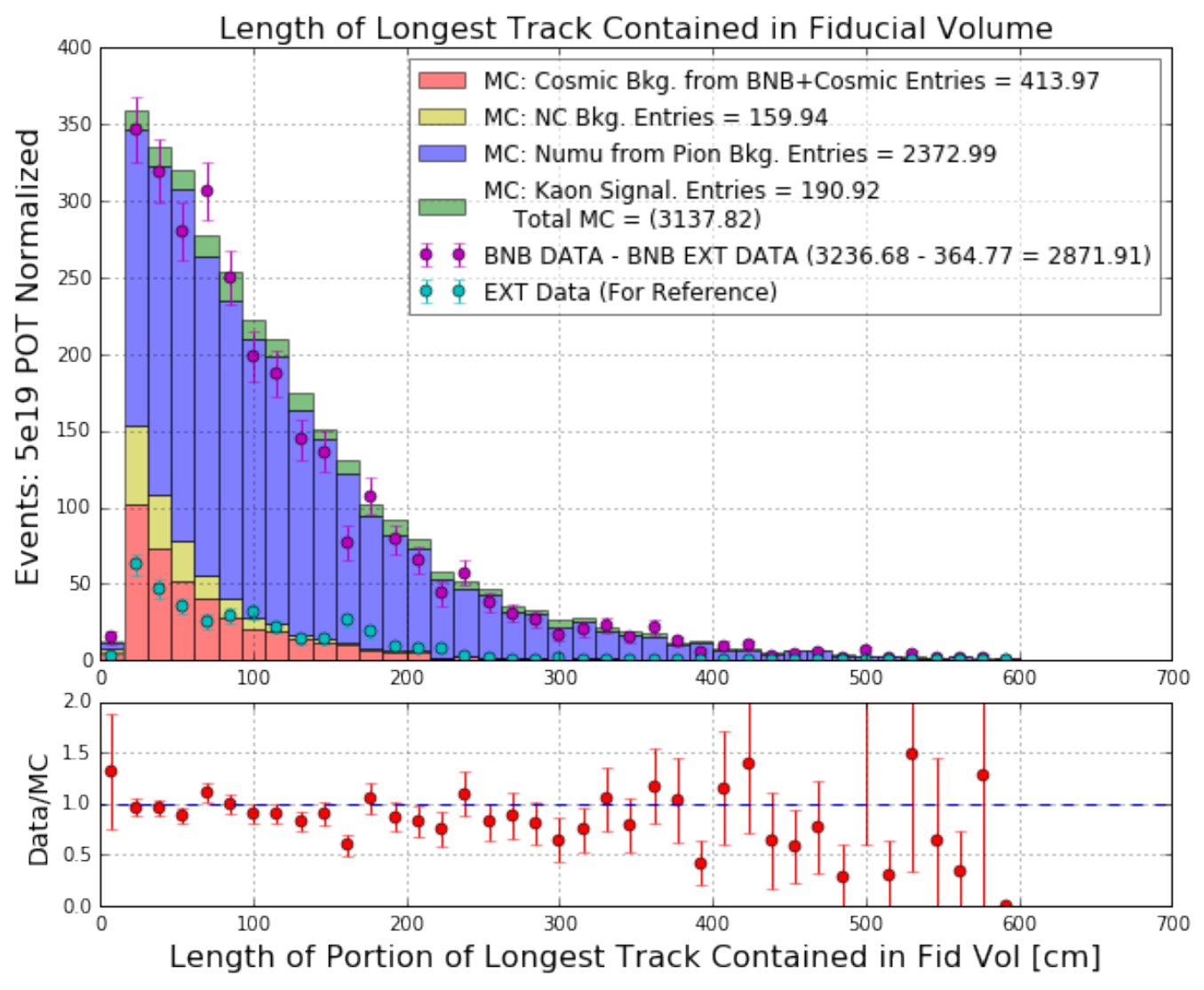

Figure 7.15: The distribution of length of the longest track associated with the interaction contained within the fiducial volume for simulated backgrounds and signal (solid histograms) overlaid with data measurements ("on-beam" minus "off-beam" drawn in purple) for the sideband region in which reconstructed neutrino energy is less than $2.5 \mathrm{GeV}$. Statistical error bars are drawn on the data points, taking into account statistics from both the "onbeam" and "off-beam" samples. Below the main figure is a bin-by-bin ratio of data divided by simulation.

the readout but a cosmic was mistakenly identified as the candidate neutrino interaction. Again, no independent studies within MicroBooNE have yet indicated that this cosmic background estimation is incorrect.

The last low level data to simulation comparison is the computed multiple Coulomb scattering (MCS) momentum of the muon track, and is shown in Figure 7.19. This distribution 


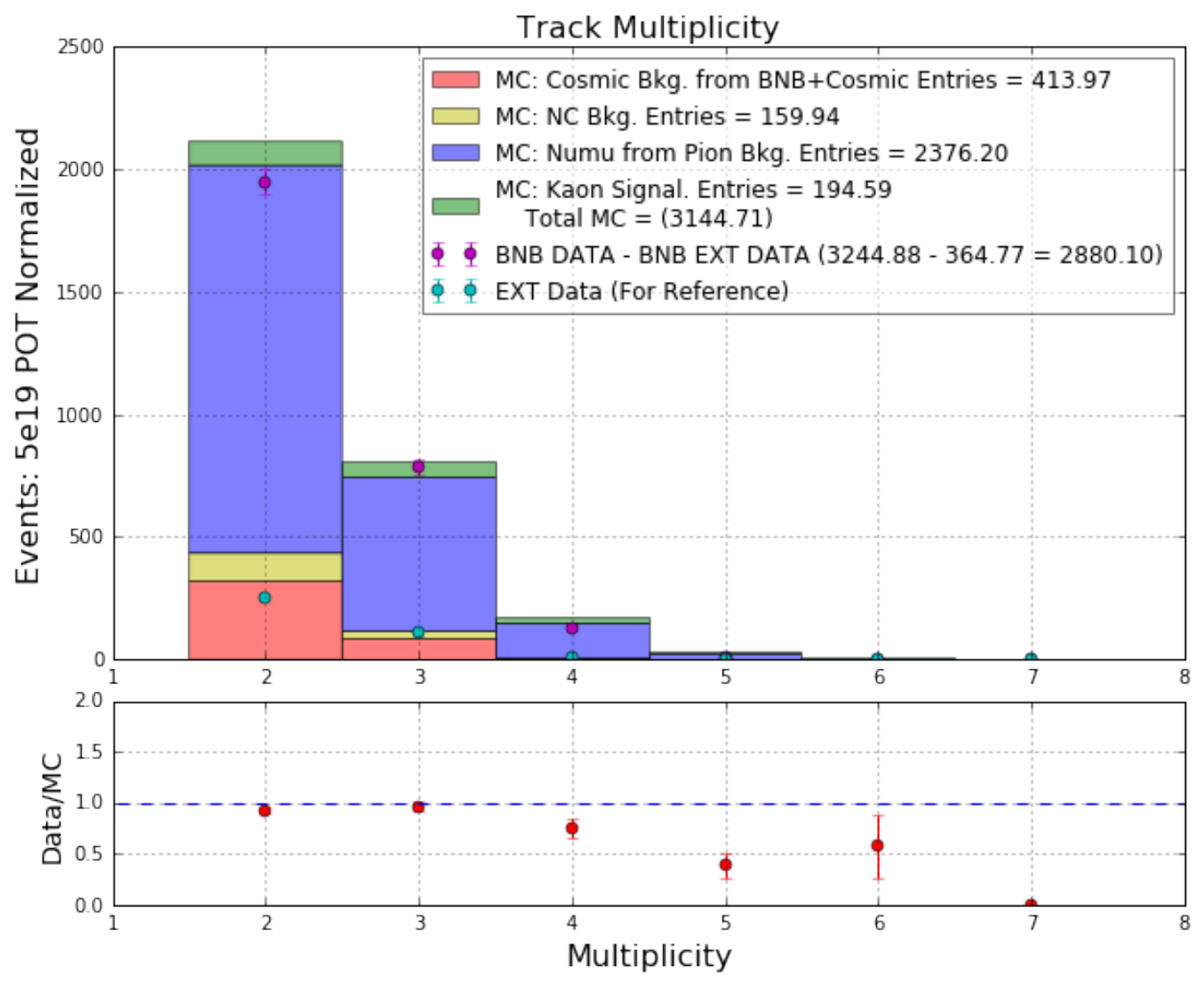

Figure 7.16: The distribution of track multiplicity (number of tracks associated with the interaction) for simulated backgrounds and signal (solid histograms) overlaid with data measurements ("on-beam" minus "off-beam" drawn in purple) for the sideband region in which reconstructed neutrino energy is less than $2.5 \mathrm{GeV}$. Statistical error bars are drawn on the data points, taking into account statistics from both the "on-beam" and "off-beam" samples. Below the main figure is a bin-by-bin ratio of data divided by simulation.

includes only tracks with at least one meter contained in the fiducial volume, as having this much track visible is necessary for the MCS technique to work. In this distribution there is a deficit of data events at higher reconstructed momenta. Since MCS momentum is a key ingredient in computing the neutrino energy (Section 7.3), and this analysis selects the highest energy events, this data to simulation disagreement is particularly important. For this reason, an extensive analysis of the MCS algorithm including data to simulation comparisons was conducted by the author of this thesis. This analysis improved the perfor- 

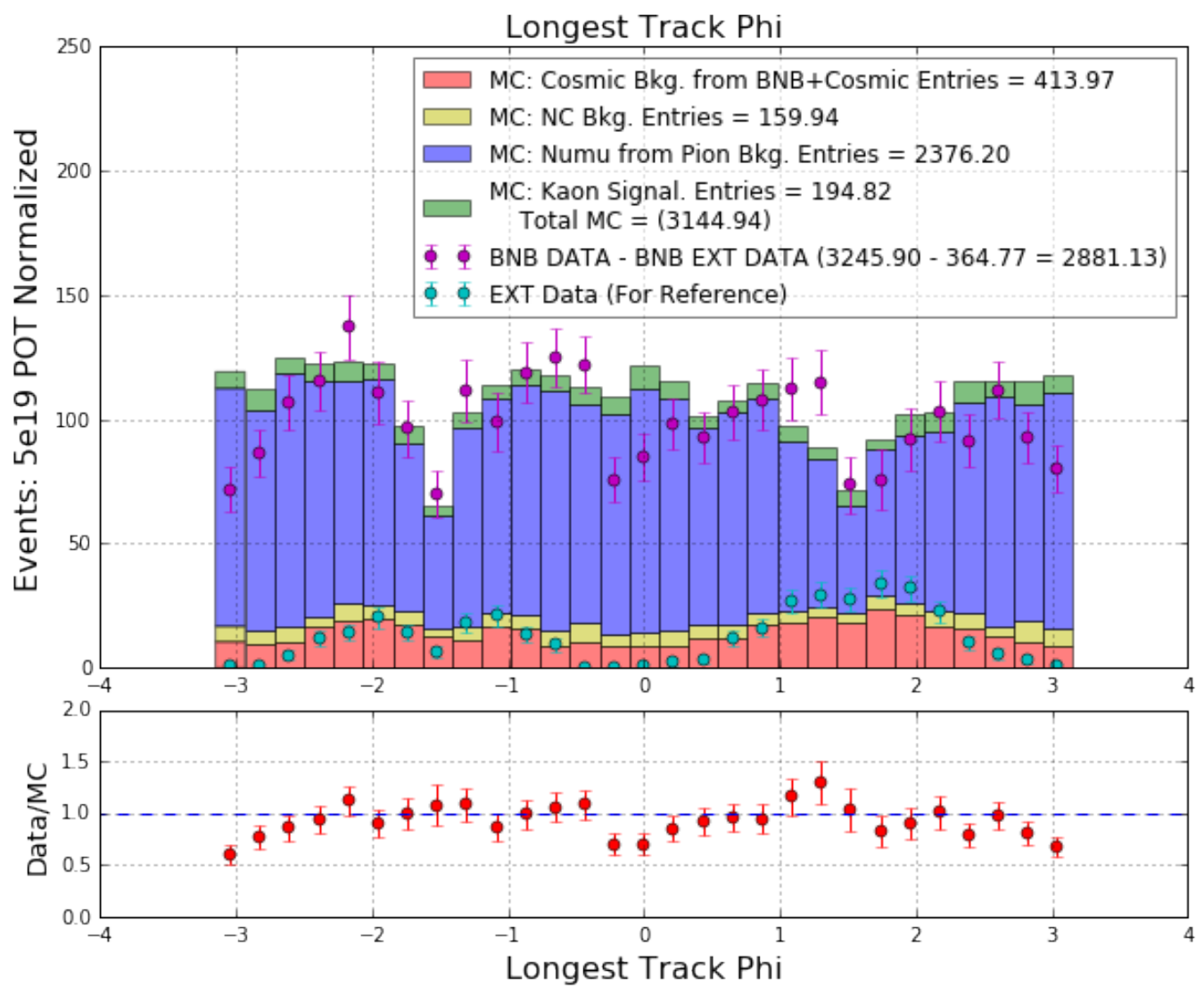

Figure 7.17: The distribution of $\phi$ angle (measured in radians with respect to the vertical) of the longest track associated with the interaction for simulated backgrounds and signal (solid histograms) overlaid with data measurements ("on-beam" minus "off-beam" drawn in purple) for the sideband region in which reconstructed neutrino energy is less than $2.5 \mathrm{GeV}$. Statistical error bars are drawn on the data points, taking into account statistics from both the "on-beam" and "off-beam" samples. Below the main figure is a bin-by-bin ratio of data divided by simulation.

mance of the algorithm, including an important change to the underlying phenomenological formula not yet discovered by other LArTPC experiments using the MCS technique. This analysis is expected to be published in the Journal of Instrumentation, and a description of this analysis is included in Appendix A of this thesis. The resulting improved MCS algorithm is the one used in the analysis described in this chapter. 


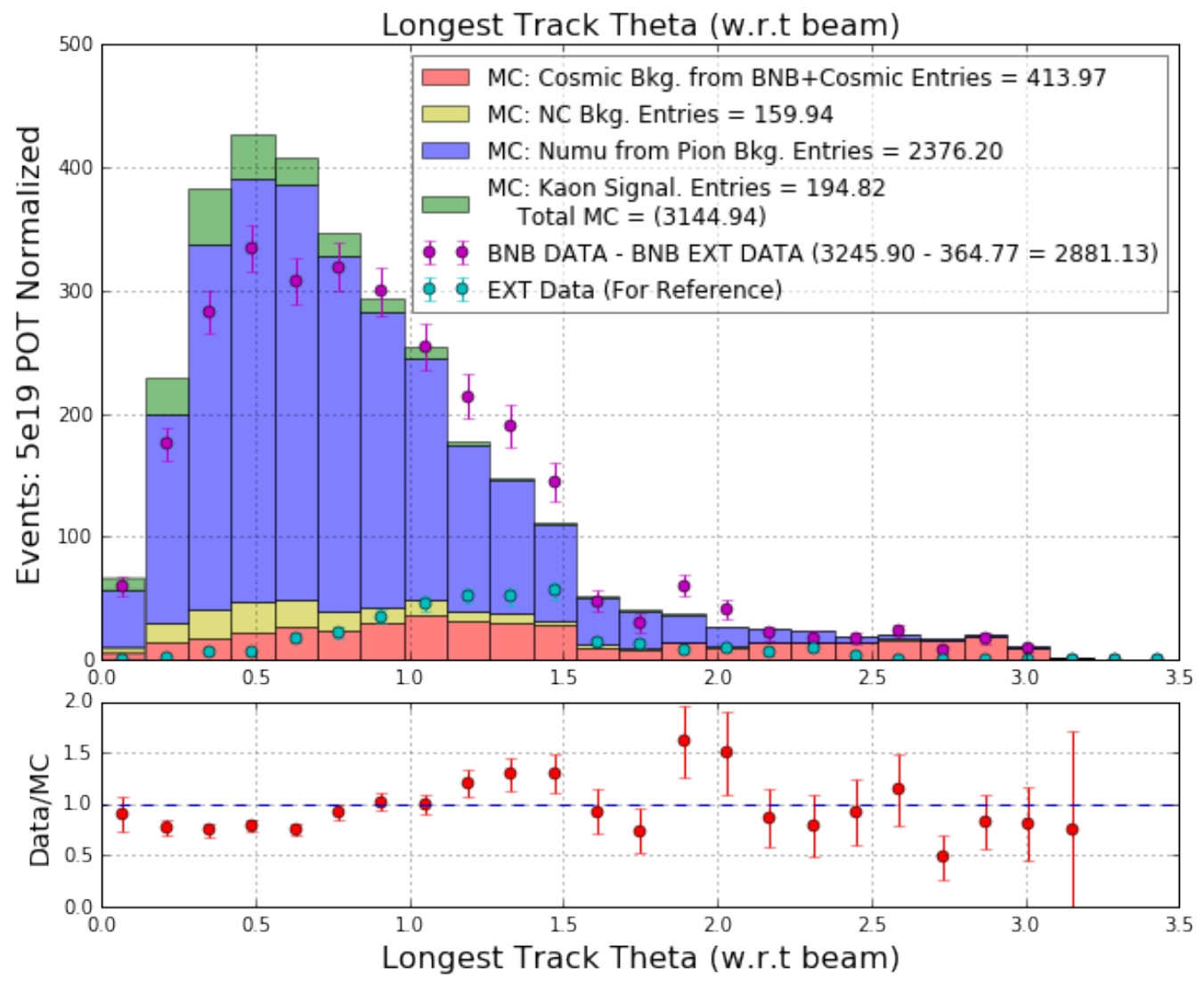

Figure 7.18: The distribution of $\theta$ angle (measured in radians with respect to the beam direction) of the longest track associated with the interaction for simulated backgrounds and signal (solid histograms) overlaid with data measurements ("on-beam" minus "off-beam" drawn in purple) for the sideband region in which reconstructed neutrino energy is less than 2.5 GeV. Statistical error bars are drawn on the data points, taking into account statistics from both the "on-beam" and "off-beam" samples. Below the main figure is a bin-by-bin ratio of data divided by simulation.

Despite extensive studies to uncover the underlying causes of the data to simulation disparities (for example the deficit of tracks at small $\theta$ angle in data and the systematic shift downward in multiple Coulomb scattering energy in data) either to fix them or calibrate them out without introducing new systematic errors, the differences remain. Since MicroBooNE is in part an $R \& D$ experiment paving the way for future LArTPC experiments, understanding these differences are a problem that the MicroBooNE collaboration is still 


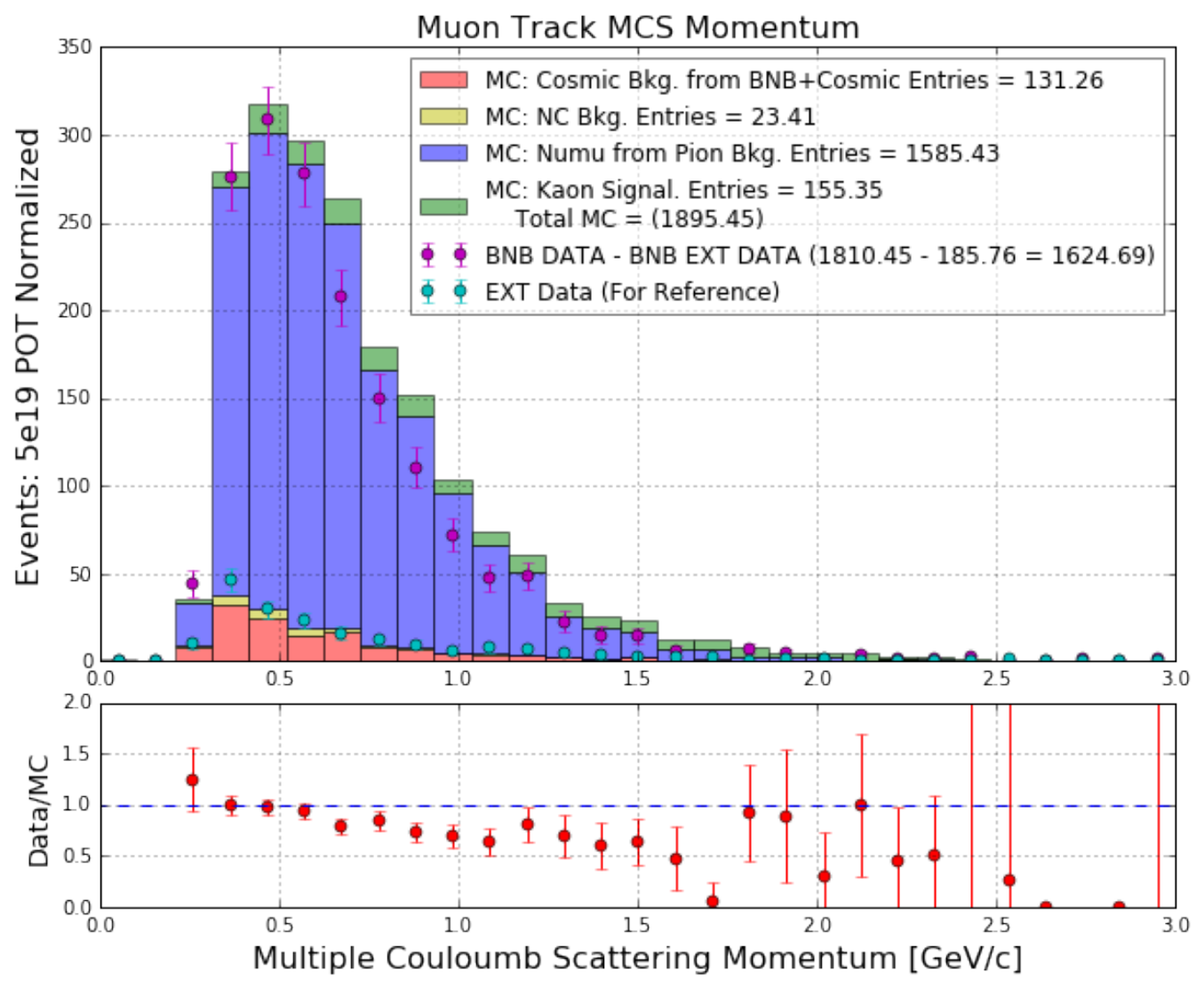

Figure 7.19: The distribution of multiple Coulomb scattering computed energy for the longest track associated with the interaction for simulated backgrounds and signal (solid histograms) overlaid with data measurements ("on-beam" minus "off-beam" drawn in purple) for the sideband region in which reconstructed neutrino energy is less than $2.5 \mathrm{GeV}$. Statistical error bars are drawn on the data points, taking into account statistics from both the "onbeam" and "off-beam" samples. Below the main figure is a bin-by-bin ratio of data divided by simulation. This plot has fewer entries than previous plots because only tracks longer than 1 meter have an associated MCS momentum as described in Section 7.3.

working to solve.

The comparison of data to simulation in terms of reconstructed neutrino energy for the sideband region with $E_{\nu}<2.5 \mathrm{GeV}$ is shown in Figure 7.20. Given the clear systematic shift in energy to lower values in data, any measurement coming from the signal region 
(which is a small tail at high energies) will have an extremely large systematic uncertainty associated with it. This figure is zoomed in on reconstructed neutrino energies between 1.5 $\mathrm{GeV}$ and $2.5 \mathrm{GeV}$ in Figure 7.21 .

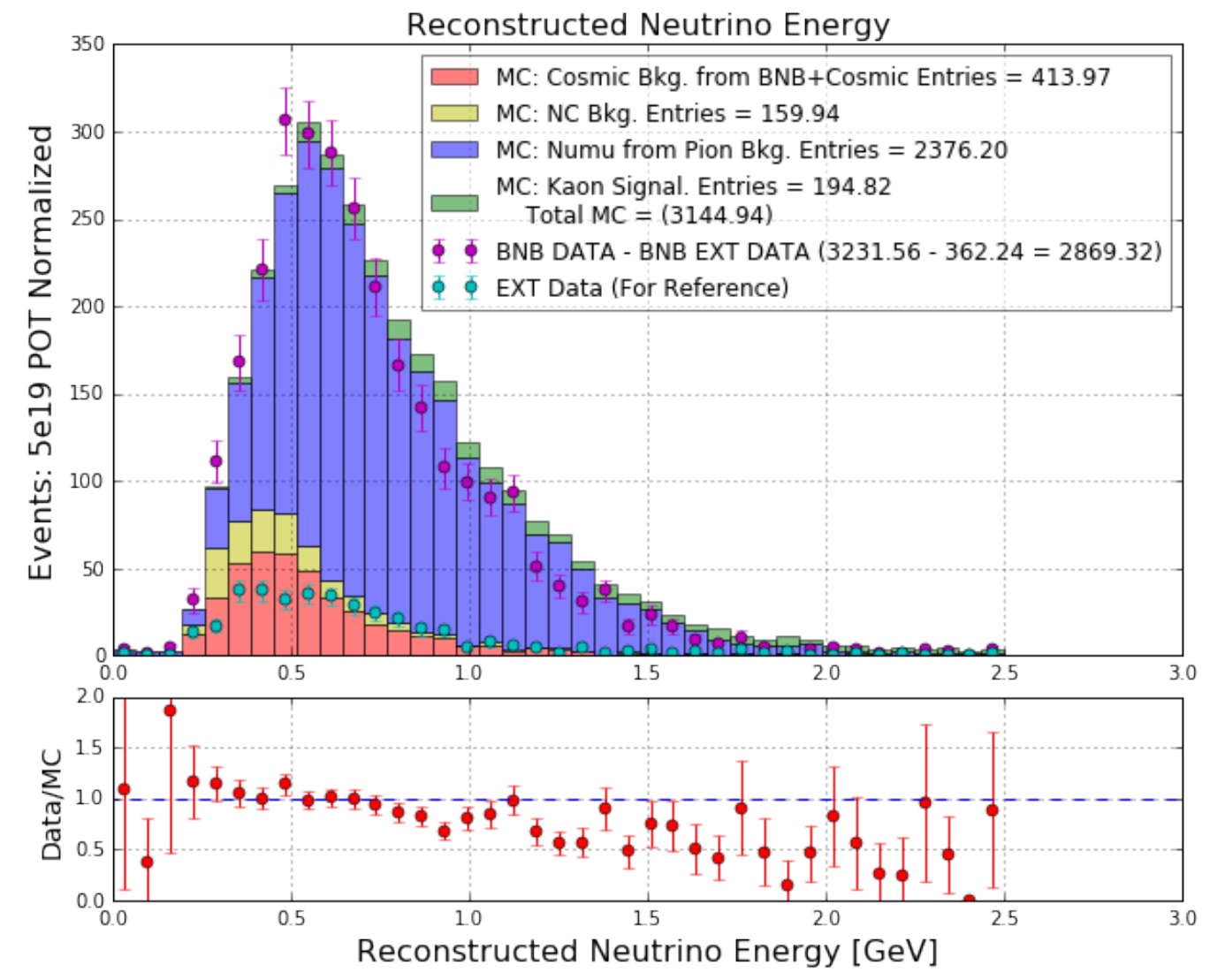

Figure 7.20: The distribution of reconstructed neutrino energy for simulated backgrounds and signal (solid histograms) overlaid with data measurements ("on-beam" minus "off-beam" drawn in purple) for the sideband region in which reconstructed neutrino energy is less than $2.5 \mathrm{GeV}$. Statistical error bars are drawn on the data points, taking into account statistics from both the "on-beam" and "off-beam" samples. Below the main figure is a bin-by-bin ratio of data divided by simulation.

For completeness, the reconstructed energy in the signal region is shown in Figure 7.22 . While the referenced SciBooNE result predicts the $K^{+}$production rate in simulation is underestimated by a factor of $0.85 \pm 0.11$ [16], the underestimation of data with respect 

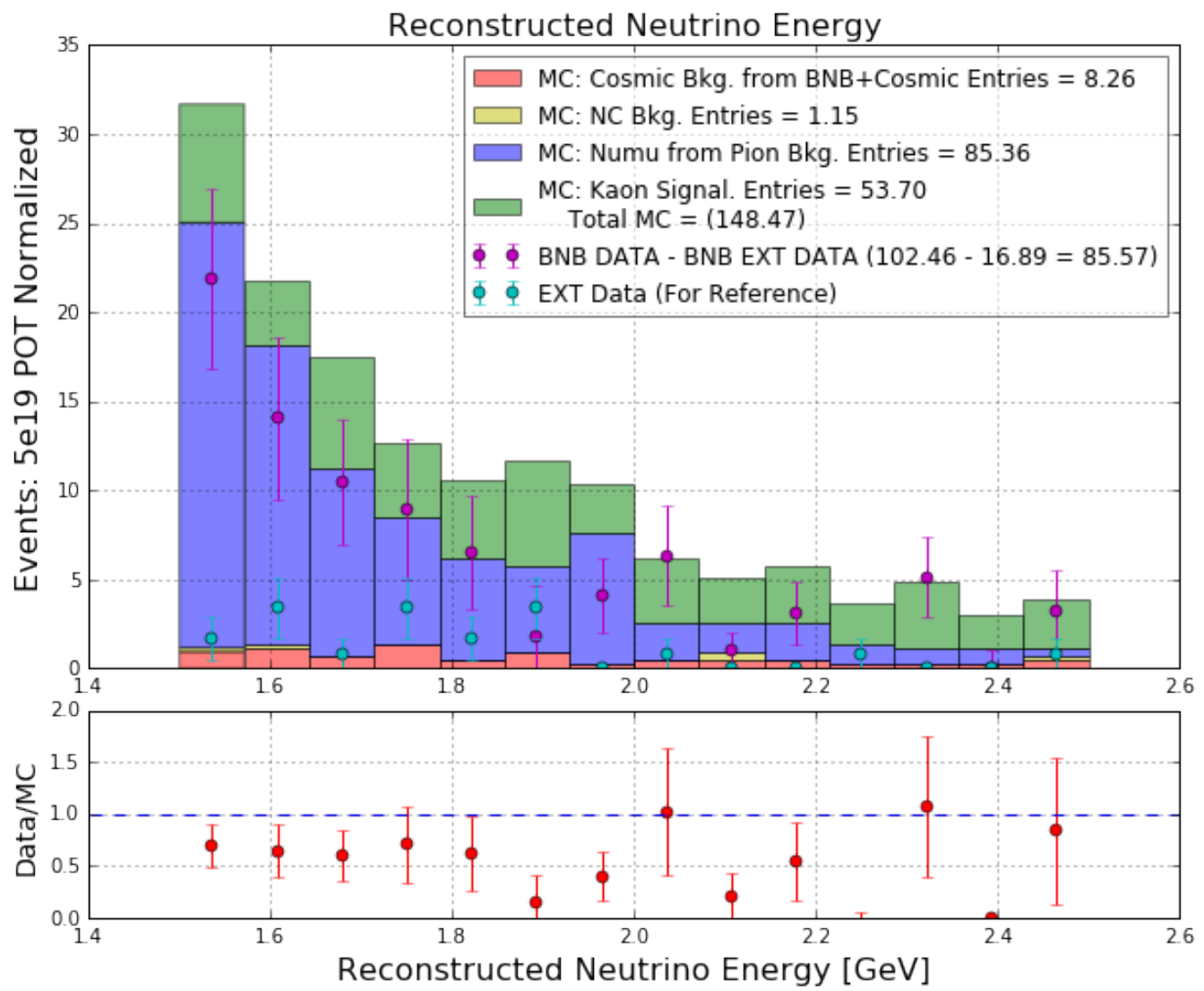

Figure 7.21: The distribution of reconstructed neutrino energy for simulated backgrounds and signal (solid histograms) overlaid with data measurements ("on-beam" minus "off-beam" drawn in purple) for the higher-energy end of the sideband region in which reconstructed neutrino energy is between 1.5 and $2.5 \mathrm{GeV}$. Statistical error bars are drawn on the data points, taking into account statistics from both the "on-beam" and "off-beam" samples. Below the main figure is a bin-by-bin ratio of data divided by simulation.

to simulation seen in this analysis is likely not indicative of incorrectly simulated kaon production in the beam-line, but instead due to systematic detector and reconstruction effects that have not yet been resolved. 

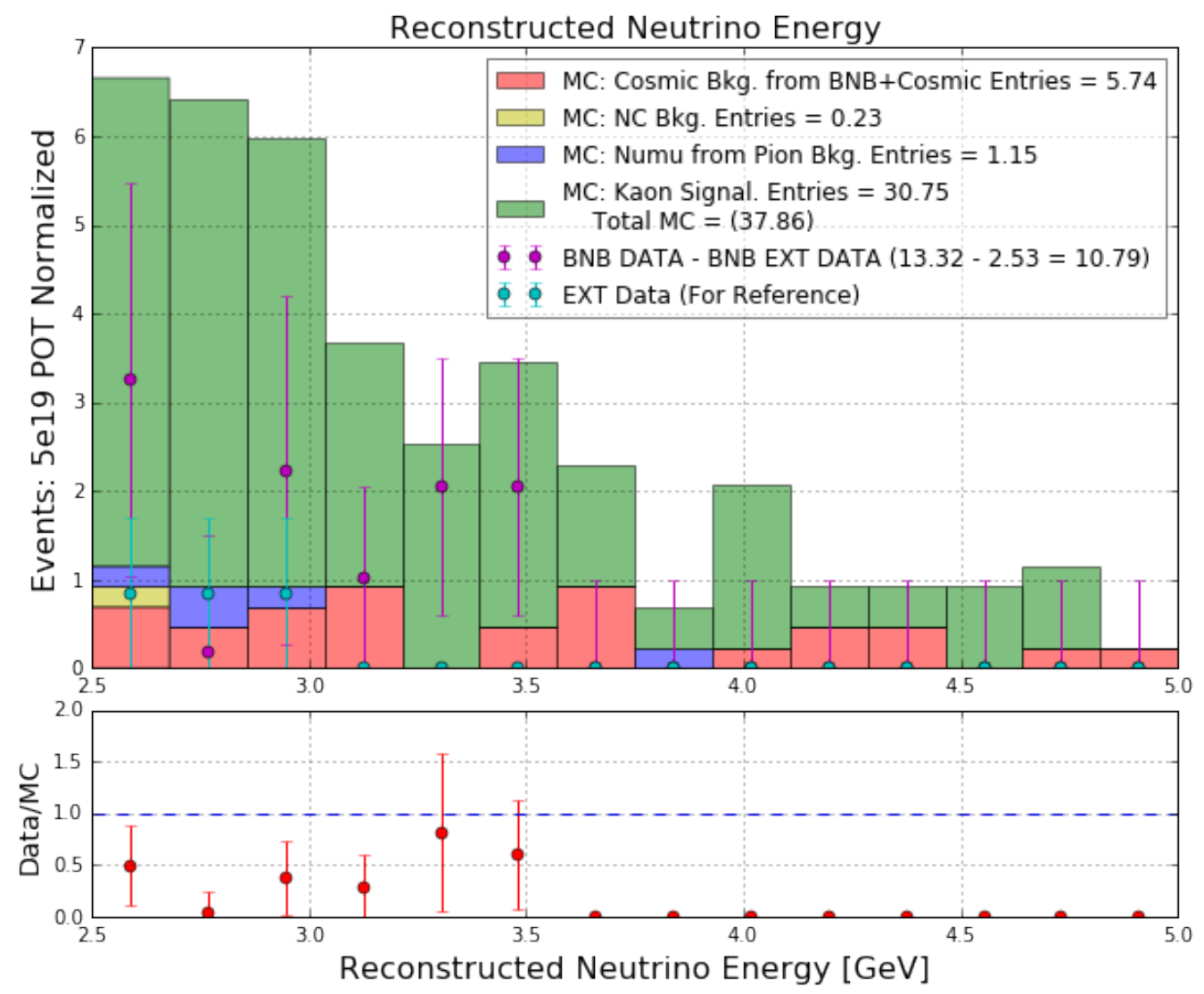

Figure 7.22: The distribution of reconstructed neutrino energy for simulated backgrounds and signal (solid histograms) overlaid with data measurements ("on-beam" minus "off-beam" drawn in purple) for the signal region in which reconstructed neutrino energy is greater than $2.5 \mathrm{GeV}$. Statistical error bars are drawn on the data points, taking into account statistics from both the "on-beam" and "off-beam" samples. Below the main figure is a bin-by-bin ratio of data divided by simulation.

\subsection{Conclusions}

This thesis chapter has presented an analysis serving as an essential first step towards measuring the kaon production in the beam-line by MicroBooNE, an important measurement used to constrain a main intrinsic $\nu_{e}$ background in the electron-like low energy excess search described in Chapter 6. While a similar measurement was previously done by the SciBooNE collaboration, this MicroBooNE measurement is important because it will be 
done in situ with the same detector that will search for the low energy excess. The method used in this analysis is to select the highest energy $\nu_{\mu}^{C C}$ interactions within the detector in order to obtain a pure sample of $\nu_{\mu}$ from $K^{+}$decay. The method was demonstrated to be viable in order to make a measurement with comparable significance as that of SciBooNE. As of now, some unresolved discrepancies between data and simulation prevent the analysis from coming to fruition. These differences are still being investigated by the MicroBooNE collaboration, and once they are understood this important analysis will proceed. Additional items left to be resolved before this kaon production systematic can be estimated in MicroBooNE aside from understanding the aforementioned data to simulation discrepancies include more thorough estimates of detector systematics, cross-section systematics, and the unblinding of more MicroBooNE data for this analysis. All of these tasks are currently being investigated within the MicroBooNE collaboration at the time this thesis was written.

With respect to these data to simulation discrepancies discussed in Section 7.5 above, one of the most important discrepancies lies within the calculation of muon momentum via multiple Coulomb scattering (MCS). Since MicroBooNE has no magnetic field, this MCS momentum determination method is the only technique by which the momentum of a muon that exits the TPC can be calculated, and the muons from the high neutrino energy interactions in the kaon enriched signal sample are all exiting. A detailed study of the MCS algorithm was conducted by the author of this thesis and has been submitted for publication to the Journal of Instrumentation (JINST). The analysis and results associated with this work is presented in Appendix $\mathrm{A}$ of this thesis. 


\section{Chapter 8}

\section{Conclusions}

Following some introductory material including a description of neutrinos and neutrino oscillations, the liquid argon time projection technique and specifically the MicroBooNE detector at the Fermi National Accelerator Lab, and the results and implications of the LSND and MiniBooNE experiments, three subsequent MicroBooNE analyses have been described. All three of these analyses are closely interconnected, and are ultimately geared towards searching for and understanding the MiniBooNE measured low energy excess of electromagnetic events in the MicroBooNE detector.

First, a detailed sensitivity analysis was described. It involved simulating beam neutrino events in the MicroBooNE detector, and using reconstruction algorithms to select them. These algorithms included the leveraging of 3D energy deposition $(d E / d x)$ information to separate electrons from photons, a capability which MiniBooNE did not possess. Additionally, MiniBooNE low energy excess public data releases were used to simulate what the excess would look like in MicroBooNE, assuming it was induced by an excess of beam $\nu_{e}$ interactions. Ultimately, a sensitivity was estimated for the MicroBooNE detector to observe such a signal. The dominant background in this search was (irreducible) intrinsic $\nu_{e}$ from both pion and kaon decay in the beam-line, and the flux uncertainty associated with the production of these particles is large.

The first steps toward the analysis to measure the $K^{+}$production in the beam-line 
by selecting high energy $\nu_{\mu}$ events was presented. This analysis constrains the significant portion of intrinsic $\nu_{e}$ backgrounds which come from kaon decay in the beam-line. This important measurement was initially done by the SciBooNE collaboration. Measuring the $K^{+}$production in MicroBooNE is relevant because the MicroBooNE detector has a different neutrino target material (argon) than SciBooNE (polystyrene), and the $K^{+}$production measurement can be done in situ in the same detector searching for the low energy excess.

An important part of the $K^{+}$analysis involves measuring the energy of muons created in $\nu_{\mu}$ interactions which exit the detector. While measuring the energy of fully contained muons is straightforward with calorimetric or range-based techniques, the only method to measure that of exiting tracks is by means of multiple Coulomb scattering (MCS). A detailed investigation into how the MCS algorithm employed by MicroBooNE works and quantification of its performance both in data and simulation was given as an Appendix.

The future prospects for MicroBooNE are bright. As of the time this thesis was written, the experiment has collected roughly $80 \%$ of the nominal protons-on-target agreed to be delivered by Fermilab. While the collaboration is continuing to develop algorithms to automatically reconstruct the data, an effort is being made to analyze data with deep learning convolutional neural networks 37. This may ultimately be MicroBooNE's path towards measuring the MiniBooNE low energy excess. Additionally, the MicroBooNE detector will serve as one of three detectors in the Short Baseline Neutrino experiment 30. which promises to deliver the most sensitive search to date for sterile neutrinos at the $\mathrm{eV}$ mass-scale. This is an exciting time for precision neutrino measurements! 


\section{Bibliography}

[1] J Chadwick. Intensittsverteilung im magnetischen Spectrum der $\beta$-Strahlen von radium B + C. Verhandl. Dtsc. Phys. Ges., 16:383, 1914.

[2] W. Pauli. Open letter to the group of radioactive people at the gauverein meeting in tubingen. 1930.

[3] S. Schael et al. Precision electroweak measurements on the $Z$ resonance. Phys. Rept., 427:257-454, 2006.

[4] Y. Fukuda et al. Evidence for oscillation of atmospheric neutrinos. Phys. Rev. Lett., 81:1562-1567, 1998.

[5] Q. R. Ahmad and others. Measurement of the rate of $\nu_{e}+d \rightarrow p+p+e^{-}$interactions produced by ${ }^{8} b$ solar neutrinos at the sudbury neutrino observatory. Phys. Rev. Lett., 87:071301, Jul 2001.

[6] X. Qian and P. Vogel. Neutrino Mass Hierarchy. Prog. Part. Nucl. Phys., 83:1-30, 2015 .

[7] S. Abe et al. Precision Measurement of Neutrino Oscillation Parameters with KamLAND. Phys. Rev. Lett., 100:221803, 2008.

[8] A. Aguilar-Arevalo et al. Evidence for neutrino oscillations from the observation of antineutrino(electron) appearance in a anti-neutrino(muon) beam. Phys. Rev., D64:112007, 2001.

[9] G. Cheng. Precision search for muon antineutrino disappearance oscillations using a dual baseline technique. 2013. 
[10] R. Acciarri et al. Design and Construction of the MicroBooNE Detector. Submitted to: JINST, 2016.

[11] A. A. Aguilar-Arevalo et al. The Neutrino Flux prediction at MiniBooNE. Phys. Rev., D79:072002, 2009.

[12] S. Agostinelli et al. GEANT4: A Simulation toolkit. Nucl. Instrum. Meth., A506:250303, 2003.

[13] M. G. Catanesi et al. Measurements of the production cross-section of positive pions in the collision of $8.9 \mathrm{GeV} / \mathrm{c}$ protons on beryllium. Eur. Phys. J., C52:29-53, 2007.

[14] I. Chemakin et al. Centrality dependence of pi- production and stopping in $\mathrm{p}$ - A collisions at 18-GeV/c. Submitted to: Phys. Rev. Lett, 1999.

[15] C. Mariani, G. Cheng, J. M. Conrad, and M. H. Shaevitz. Improved Parameterization of $K^{+}$Production in p-Be Collisions at Low Energy Using Feynman Scaling. Phys. Rev., D84:114021, 2011.

[16] G. Cheng et al. Measurement of $K^{+}$production cross section by $8 \mathrm{GeV}$ protons using high energy neutrino interactions in the SciBooNE detector. Phys. Rev., D84:012009, 2011.

[17] A. A. Aguilar-Arevalo et al. The MiniBooNE Detector. Nucl. Instrum. Meth., A599:2846, 2009.

[18] G. Karagiorgi. Searches for new physics at miniboone: Sterile neutrinos and mixing freedom. 2010.

[19] A. A. Aguilar-Arevalo et al. First Measurement of the Muon Neutrino Charged Current Quasielastic Double Differential Cross Section. Phys. Rev., D81:092005, 2010.

[20] A. A. Aguilar-Arevalo et al. A Search for electron neutrino appearance at the $\Delta m^{2} \sim$ 1eV² scale. Phys. Rev. Lett., 98:231801, 2007.

[21] A. A. Aguilar-Arevalo et al. Unexplained Excess of Electron-Like Events From a 1-GeV Neutrino Beam. Phys. Rev. Lett., 102:101802, 2009. 
[22] Thomas Schwetz. The LSND puzzle in the light of MiniBooNE results. In Proceedings, 43rd Rencontres de Moriond on Electroweak Interactions and Unified Theories: La Thuile, Italy, March 1-8, 2008, 2008.

[23] H. Chen et al. A Proposal for a New Experiment Using the Booster and NuMI Neutrino Beamlines: MicroBooNE.

[24] The microboone technical design report. 2012.

[25] Costas Andreopoulos, Christopher Barry, Steve Dytman, Hugh Gallagher, Tomasz Golan, Robert Hatcher, Gabriel Perdue, and Julia Yarba. The GENIE Neutrino Monte Carlo Generator: Physics and User Manual. 2015.

[26] D. Casper. The Nuance neutrino physics simulation, and the future. Nucl. Phys. Proc. Suppl., 112:161-170, 2002. [,161(2002)].

[27] D. Heck, G. Schatz, T. Thouw, J. Knapp, and J. N. Capdevielle. CORSIKA: A Monte Carlo code to simulate extensive air showers. 1998.

[28] Wouter Verkerke and David P. Kirkby. The RooFit toolkit for data modeling. eConf, C0303241:MOLT007, 2003. [,186(2003)].

[29] A. A. Aguilar-Arevalo et al. Unexplained Excess of Electron-Like Events From a 1-GeV Neutrino Beam (Data Release).

[30] M. Antonello et al. A Proposal for a Three Detector Short-Baseline Neutrino Oscillation Program in the Fermilab Booster Neutrino Beam. 2015.

[31] A. Ankowski et al. Energy reconstruction of electromagnetic showers from pi0 decays with the ICARUS T600 Liquid Argon TPC. Acta Phys. Polon., B41:103-125, 2010.

[32] J. R. Sanford and C. L. Wang. BNL Internal Note No, 11299, 1967.

[33] J. S. Marshall and M. A. Thomson. The Pandora Software Development Kit for Pattern Recognition. Eur. Phys. J., C75(9):439, 2015.

[34] R. Acciarri et al. Study of Space Charge Effects in MicroBooNE. 
[35] N. V. Mokhov D. E. Groom and S. Striganov. Muon Stopping Power and Range Tables: $10 \mathrm{MeV}-100 \mathrm{TeV}$, Table 5.

[36] Muons in liquid argon (ar) table 289.

[37] R. Acciarri et al. Convolutional Neural Networks Applied to Neutrino Events in a Liquid Argon Time Projection Chamber. Submitted to: JINST, 2016. 


\section{Part I}

\section{Appendices}


Appendix A

Multiple Coulomb Scattering

Publication 


\title{
Determination of muon momentum in the
} MicroBooNE LArTPC using an improved model of multiple Coulomb scattering

\author{
P. Abratenko ${ }^{n}$ R. Acciarri ${ }^{g}$ C. Adams ${ }^{b b}$ R. An ${ }^{h}$ J. Anthony ${ }^{c}$ J. Asaadi ${ }^{y}$ M. Auger ${ }^{a}$ \\ L. Bagby ${ }^{g}$ S. Balasubramanian ${ }^{b b}$ B. Baller ${ }^{g}$ C. Barnes ${ }^{n}$ G. Barr ${ }^{q}$ M. Bass ${ }^{q}$ F. Bay ${ }^{z}$ \\ M. Bishai ${ }^{b}$ A. Blake ${ }^{j}$ T. Bolton ${ }^{i}$ L. Bugel ${ }^{m}$ L. Camilleri $^{f}$ D. Caratelli ${ }^{f}$ B. Carls ${ }^{g}$ \\ R. Castillo Fernandez ${ }^{g}$ F. Cavanna ${ }^{g}$ H. Chen ${ }^{b}$ E. Church $^{r}$ D. Ciancil ${ }^{l}, f$ E. Cohen ${ }^{w}$ \\ G. H. Collin ${ }^{m}$ J. M. Conrad ${ }^{m}$ M. Convery ${ }^{u}$ J. I. Crespo-Anadón ${ }^{f}$ M. Del Tutto ${ }^{q}$ \\ D. Devitt ${ }^{j}$ S. Dytman ${ }^{s}$ B. Eberly ${ }^{u}$ A. Ereditato ${ }^{a}$ L. Escudero Sanchez $^{c}$ J. Esquivel $^{v}$ \\ B. T. Fleming ${ }^{b b}$ W. Foreman ${ }^{d}$ A. P. Furmanski ${ }^{l}$ D. Garcia-Gamez ${ }^{l}$ G. T. Garvey ${ }^{k}$ \\ V. Genty ${ }^{f}$ D. Goeldi ${ }^{a}$ S. Gollapinni ${ }^{i, x}$ N. Graf ${ }^{s}$ E. Gramellini ${ }^{b b}$ H. Greenlee ${ }^{g}$ \\ R. Grosso ${ }^{e}$ R. Guenette ${ }^{q}$ A. Hackenburg ${ }^{b b}$ P. Hamilton ${ }^{v}$ O. Hen ${ }^{m}$ J. Hewes ${ }^{l}$ C. Hill ${ }^{l}$ \\ J. Ho ${ }^{d}$ G. Horton-Smith ${ }^{i}$ E.-C. Huang ${ }^{k}$ C. James ${ }^{g}$ J. Jan de Vries ${ }^{c}$ C.-M. Jen $a a$ \\ L. Jiang ${ }^{s}$ R. A. Johnson ${ }^{e}$ J. Joshi ${ }^{b}$ H. Jostlein ${ }^{g}$ D. Kaleko ${ }^{f}$ L. N. Kalousis ${ }^{a a, 1}$ \\ G. Karagiorgi ${ }^{l, f}$ W. Ketchum ${ }^{g}$ B. Kirby ${ }^{b}$ M. Kirby ${ }^{g}$ T. Kobilarcik ${ }^{g}$ I. Kreslo ${ }^{a}$

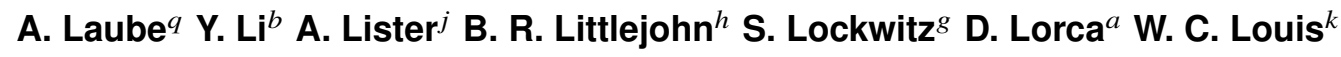 \\ M. Luethi ${ }^{a}$ B. Lundberg ${ }^{g}$ X. Luo ${ }^{b b}$ A. Marchionni ${ }^{g}$ C. Mariani ${ }^{a a}$ J. Marshall $^{c}$ \\ D. A. Martinez Caicedo ${ }^{h}$ V. Meddage ${ }^{i}$ T. Miceli $^{o}$ G. B. Mills ${ }^{k}$ J. Moon $^{m}$ M. Mooney ${ }^{b}$ \\ C. D. Moore ${ }^{g}$ J. Mousseau ${ }^{n}$ R. Murrells ${ }^{l}$ D. Naples ${ }^{s}$ P. Nienaber ${ }^{t}$ J. Nowak ${ }^{j}$ \\ O. Palamara ${ }^{g}$ V. Paolone ${ }^{s}$ V. Papavassiliou $^{o}$ S. F. Pate ${ }^{o}$ Z. Pavlovic ${ }^{g}$ E. Piasetzky ${ }^{w}$ \\ D. Porzio ${ }^{l}$ G. Pulliam ${ }^{v}$ X. Qian ${ }^{b}$ J. L. Raaf ${ }^{g}$ A. Rafique ${ }^{i}$ L. Rochester ${ }^{u}$ \\ C. Rudolf von Rohr ${ }^{a}$ B. Russell ${ }^{b b}$ D. W. Schmitz ${ }^{d}$ A. Schukraft ${ }^{g}$ W. Seligman ${ }^{f}$ \\ M. H. Shaevitz ${ }^{f}$ J. Sinclair $^{a}$ E. L. Snider ${ }^{g}$ M. Soderberg ${ }^{v}$ S. Söldner-Rembold ${ }^{l}$ \\ S. R. Soleti ${ }^{q}$ P. Spentzouris ${ }^{g}$ J. Spitz ${ }^{n}$ J. St. John ${ }^{e}$ T. Strauss ${ }^{g}$ A. M. Szelc ${ }^{l}$ N. Tagg $^{p}$ \\ K. Terao ${ }^{f}$ M. Thomson ${ }^{c}$ M. Toups ${ }^{g}$ Y.-T. Tsai $^{u}$ S. Tufanli ${ }^{b b}$ T. Usher $^{u}$ \\ R. G. Van de Water ${ }^{k}$ B. Viren ${ }^{b}$ M. Weber ${ }^{a}$ D. A. Wickremasinghe ${ }^{s}$ S. Wolbers ${ }^{g}$ \\ T. Wongjirad ${ }^{m}$ K. Woodruff ${ }^{o}$ T. Yang $^{g}{\text { L. } \text { Yates }^{m} \text { G. P. Zeller }}^{g}$ J. Zennamo $^{d}$ C. Zhang ${ }^{b}$ \\ ${ }^{a}$ Universität Bern, Bern CH-3012, Switzerland \\ ${ }^{b}$ Brookhaven National Laboratory (BNL), Upton, NY, 11973, USA \\ ${ }^{c}$ University of Cambridge, Cambridge CB3 OHE, United Kingdom \\ ${ }^{d}$ University of Chicago, Chicago, IL, 60637, USA \\ ${ }^{e}$ University of Cincinnati, Cincinnati, OH, 45221, USA \\ ${ }^{f}$ Columbia University, New York, NY, 10027, USA \\ ${ }^{g}$ Fermi National Accelerator Laboratory (FNAL), Batavia, IL 60510, USA
}

${ }^{1}$ now at: Vrije Universiteit Brussel 
${ }^{i}$ Kansas State University (KSU), Manhattan, KS, 66506, USA

${ }^{j}$ Lancaster University, Lancaster LA1 4YW, United Kingdom

${ }^{k}$ Los Alamos National Laboratory (LANL), Los Alamos, NM, 87545, USA

${ }^{l}$ The University of Manchester, Manchester M13 9PL, United Kingdom

${ }^{m}$ Massachusetts Institute of Technology (MIT), Cambridge, MA, 02139, USA

${ }^{n}$ University of Michigan, Ann Arbor, MI, 48109, USA

${ }^{\circ}$ New Mexico State University (NMSU), Las Cruces, NM, 88003, USA

${ }^{p}$ Otterbein University, Westerville, OH, 43081, USA

${ }^{q}$ University of Oxford, Oxford OX1 3RH, United Kingdom

${ }^{r}$ Pacific Northwest National Laboratory (PNNL), Richland, WA, 99352, USA

${ }^{s}$ University of Pittsburgh, Pittsburgh, PA, 15260, USA

${ }^{t}$ Saint Mary's University of Minnesota, Winona, MN, 55987, USA

${ }^{u}$ SLAC National Accelerator Laboratory, Menlo Park, CA, 94025, USA

${ }^{v}$ Syracuse University, Syracuse, NY, 13244, USA

${ }^{w}$ Tel Aviv University, Tel Aviv, Israel, 69978

${ }^{x}$ University of Tennessee, Knoxville, TN, 37996, USA

${ }^{y}$ University of Texas, Arlington, TX, 76019, USA

${ }^{z}$ TUBITAK Space Technologies Research Institute, METU Campus, TR-06800, Ankara, Turkey

${ }^{a a}$ Center for Neutrino Physics, Virginia Tech, Blacksburg, VA, 24061, USA

${ }^{b b}$ Yale University, New Haven, CT, 06520, USA

Aвstract: We discuss a technique for measuring a charged particle's momentum by means of multiple Coulomb scattering (MCS) in the MicroBooNE liquid argon time projection chamber (LArTPC). This method does not require the full particle ionization track to be contained inside of the detector volume as other track momentum reconstruction methods do (range-based momentum reconstruction and calorimetric momentum reconstruction). We motivate use of this technique, describe a tuning of the underlying phenomenological formula, quantify its performance on fully contained beam-neutrino-induced muon tracks both in simulation and in data, and quantify its performance on exiting muon tracks in simulation. We find agreement between data and simulation for contained tracks, with a small bias in the momentum reconstruction and with resolutions that vary as a function of track length, improving from about $10 \%$ for the shortest (one meter long) tracks to 5\% for longer (several meter) tracks. For simulated exiting muons with at least one meter of track contained, we find a similarly small bias, and a resolution which is less than $15 \%$ for muons with momentum below $2 \mathrm{GeV} / \mathrm{c}$. 


\section{Contents}

1 Introduction and motivation 1

2 Multiple Coulomb scattering 2

2.1 Tuning the Highland formula for argon 4

3 MCS implementation using the maximum likelihood method 6

$\begin{array}{lll}3.1 \text { Track segmentation and scattering angle computation } & 7\end{array}$

3.2 Maximum likelihood theory $\quad 7$

3.3 Maximum likelihood implementation $\quad 8$

4 Range-based energy validation from simulation $\quad 8$

5 MCS performance on beam neutrino-induced muons in MicroBooNE data 10

$\begin{array}{ll}5.1 \text { Input sample } & 10\end{array}$

$\begin{array}{ll}5.2 \text { Event selection } & 10\end{array}$

5.3 Validation of the Highland formula 11

$\begin{array}{lll}5.4 & \text { MCS momentum validation } & 11\end{array}$

5.5 Impact of Highland formula tuning $\quad 12$

6 MCS performance on exiting muons in MicroBooNE simulation 15

$\begin{array}{lll}7 & \text { Conclusions } & 16\end{array}$

\section{Introduction and motivation}

In this paper we summarize the theory of multiple Coulomb scattering (MCS) and describe how the underlying Highland formula is retuned based on Monte Carlo simulation for use in liquid-argon time-projection chambers (LArTPCs). We present a maximum likelihood based algorithm that is used to determine the momentum of particles in a LArTPC. The only way to determine the momentum of a particle that exits the active volume of a LArTPC is through MCS measurements. We demonstrate that this technique works well for a sample of fully contained muons from Booster Neutrino Beam (BNB) $v_{\mu}$ charged-current (CC) interactions, and determine the resolutions and biases of the measurement. In addition we demonstrate the performance of the method on simulated exiting tracks.

MicroBooNE (Micro Booster Neutrino Experiment) is an experiment that uses a large LArTPC to investigate the excess of low energy events observed by the MiniBooNE experiment [1] and to study neutrino-argon cross-sections. MicroBooNE is the first detector of the Short-Baseline Neutrino (SBN) [2] physics program at the Fermi National Accelerator Laboratory (Fermilab), to be 
And Rare Underground Signal (ICARUS) detector [3]. In addition to producing valuable physics output, MicroBooNE serves as an important source of detector and reconstruction development for future LArTPC experiments, such as the Deep Underground Neutrino Experiment (DUNE) [4].

The MicroBooNE detector [5] consists of a rectangular time-projection chamber (TPC) with dimensions $2.6 \mathrm{~m} \times 2.3 \mathrm{~m} \times 10.4 \mathrm{~m}$ (width $\times$ height $\times$ length) located $470 \mathrm{~m}$ downstream from the Booster Neutrino Beam (BNB) target [6]. LArTPCs allow for precise three-dimensional reconstruction of particle interactions. For later reference, the $z$ axis of the detector is horizontal, along the direction of the BNB, while the $x$ direction of the TPC corresponds to the drift coordinate and the $y$ direction is the vertical direction. The mass of active liquid argon contained within the MicroBooNE TPC volume is about 90 tons, out of a total mass of 170 tons.

A set of 32 photomultiplier tubes (PMTs) and three planes of TPC wires with $3 \mathrm{~mm}$ spacing at angles of 0 , and \pm 60 degrees with respect to the vertical are used for event reconstruction. The cathode plane operating voltage is $-70 \mathrm{kV}$. As illustrated in figure 1, a neutrino in the beam interacts with an argon nucleus and the charged outgoing particles traverse the medium, lose energy and leave an ionization trail. The resulting ionization electrons drift in a $273 \mathrm{~V} / \mathrm{cm}$ electric field to the wire planes constituting the anode. The passage of these electrons through the first two wire planes induces a signal in the wires, and their collection on the third plane also generates a signal. These signals are used to create three distinct two-dimensional views (in terms of wire and time) of the event. Combining these wire signals allow for full three-dimensional reconstruction of the event, with PMT signals providing information about the absolute drift $(x)$ coordinate. The boundaries of the fiducial volume used in this analysis are set back from the six faces of the active volume by distances of between 20 and $37 \mathrm{~cm}$, depending on the face, to reduce the impact of electric-field non-uniformities near the edges of the TPC. This volume corresponds to a mass of 55 tons.

The Booster Neutrino Beam (BNB) is composed predominantly of muon neutrinos $\left(v_{\mu}\right)$ with a peak neutrino energy of about $0.7 \mathrm{GeV}$. Some of these neutrinos undergo charged current $\left(v_{\mu} \mathrm{CC}\right)$ interactions in the TPC and produce muons and other particles. For muon tracks that are completely contained in the TPC, we calculate the momentum with a measurement of the length of the particle's track, or with calorimetric measurements which come from wire signal size measurements. Roughly half of the muons from BNB $v_{\mu} \mathrm{CC}$ interactions in MicroBooNE are not fully contained in the TPC, and therefore using an established length-based or calorimetry-based method to determine the momenta for these uncontained tracks is not a possibility; the only way to determine their momenta is through MCS.

\section{Multiple Coulomb scattering}

Multiple Coulomb scattering occurs when a charged particle traverses a medium and undergoes electromagnetic scattering off atomic nuclei. This scattering perturbs the original trajectory of the particle within the material (figure 2). For a given initial momentum $p$, the angular deflection scat- 


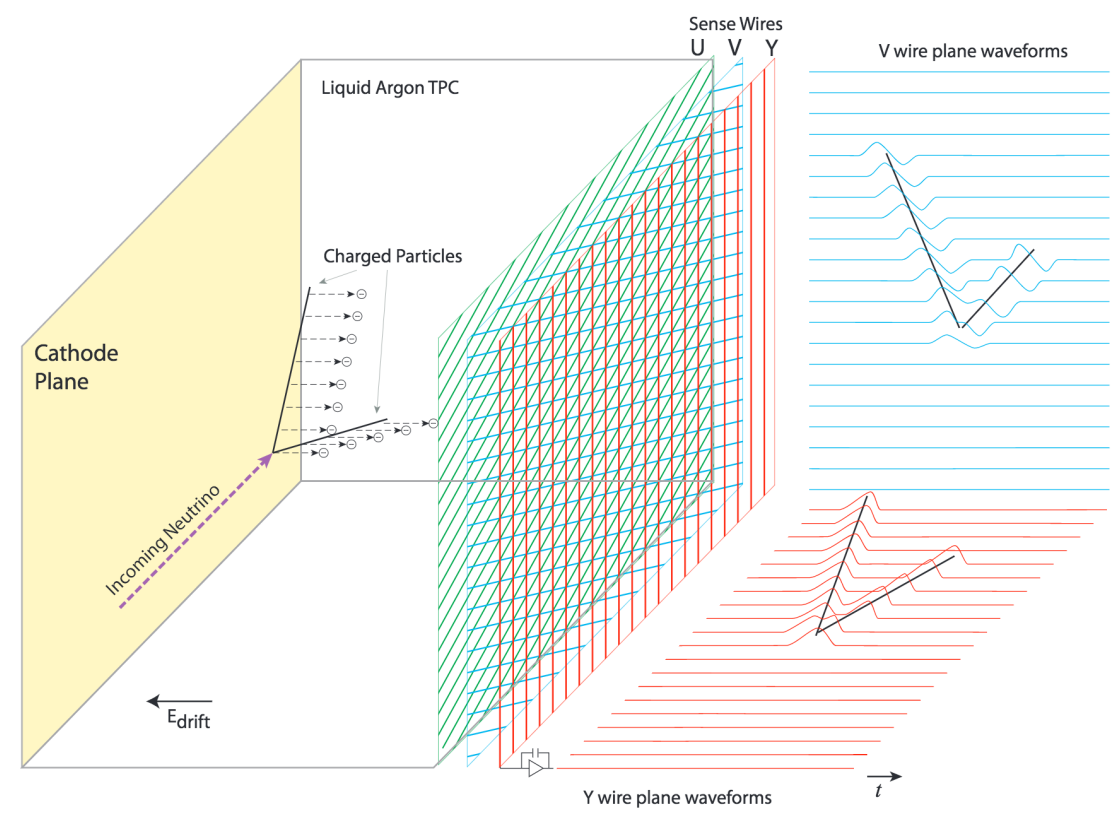

Figure 1. A diagram of the time projection chamber of the MicroBooNE detector [5]. PMTs (not shown) are located behind the wire planes.

ters of a particle in either the $x^{\prime}$ direction or $y^{\prime}$ direction (as indicated in the aforementioned figure) form a Gaussian distribution centered at zero with an RMS width, $\sigma_{o}^{\mathrm{HL}}$, given by the Highland formula $[7,8]$

$$
\sigma_{o}^{\mathrm{HL}}=\frac{S_{2}}{p \beta c} z \sqrt{\frac{\ell}{X_{0}}}\left[1+\epsilon \times \ln \left(\frac{\ell}{X_{0}}\right)\right],
$$

where $\beta$ is the ratio of the particle's velocity to the speed of light (assuming the particle is a muon), $\ell$ is the distance traveled inside the material, $z$ is the magnitude of the charge of the particle (unity, for the case of muons), and $X_{0}$ is the radiation length of the target material (taken to be a constant $14 \mathrm{~cm}$ in liquid argon). $S_{2}$ and $\epsilon$ are parameters determined to be $13.6 \mathrm{MeV}$ and 0.038 , respectively. In this study, a modified version of the Highland formula is used that includes a detector-inherent angular resolution term, $\sigma_{o}^{\text {res }}$

$$
\sigma_{o}=\sqrt{\left(\sigma_{o}^{\mathrm{HL}}\right)^{2}+\left(\sigma_{o}^{\mathrm{res}}\right)^{2}}
$$

For this analysis, the $\sigma_{o}^{\text {res }}$ term is given a fixed value of $3 \mathrm{mrad}$ which has been determined to be an acceptable value based on MicroBooNE simulation studies of muons at higher momenta. At $4.5 \mathrm{GeV} / \mathrm{c}$ muon momentum and $l \approx X_{0}$, equation 2.1 predicts an RMS angular scatter of $3 \mathrm{mrad}$, comparable to the detector resolution. The fully contained muons addressed in this analysis have 
With the Highland formula, the momentum of a track-like particle can be determined using only the 3D reconstructed track information, without any calorimetric or track range information. In neutrino physics experiments, emulsion detectors like those employed by the DONUT [9] and OPERA [10] collaborations have used MCS to determine particle momenta. Additionally, the MACRO [11] collaboration at Gran Sasso Laboratory utilized this technique. For LArTPCs, the ICARUS collaboration has described the MCS-based determination of particle momentum using a variety of methods $[12,13]$. The likelihood-based method discussed in this paper for use in the MicrobooNE detector and described in detail in section 3, has improved on the ICARUS method by tuning the underlying phenomenological formula.

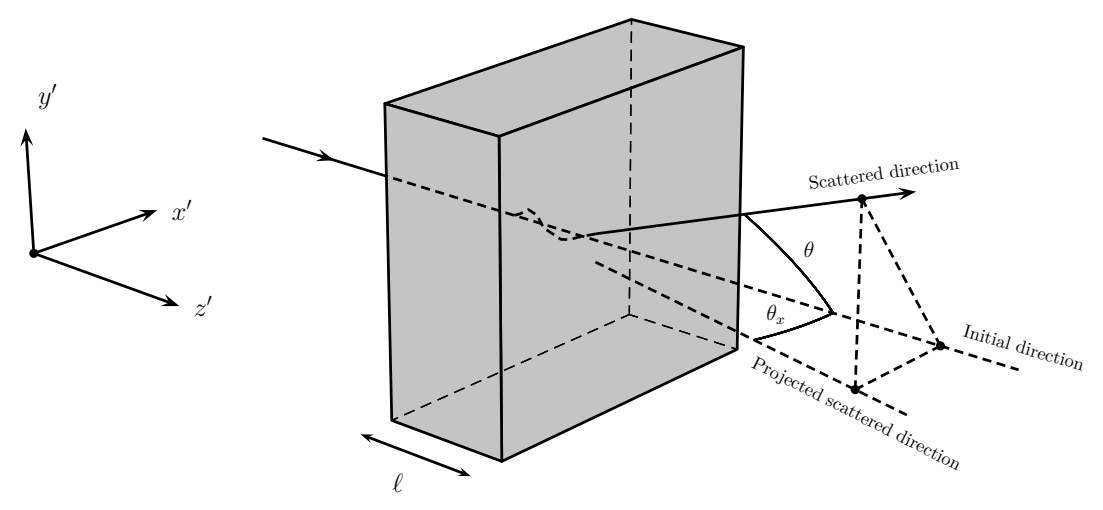

Figure 2. The particle's trajectory is deflected as it traverses the material. The angular scatter in the labeled $x^{\prime}$ direction is shown as $\theta_{x}$.

\subsection{Tuning the Highland formula for argon}

The Highland formula as written in equation 2.1 originates from a 1991 publication by G. R. Lynch and O. I. Dahl [8]. The parameters in the equation $\left(S_{2}\right.$ and $\left.\epsilon\right)$ were determined using a global fit to MCS simulated data using a modified GEANT simulation package of 14 different elements and 7 thickness ranges. All of the simulated particles were relativistic, with $\beta=1$. The materials studied ranged from hydrogen (with $\mathrm{Z}=1$ ) to uranium (with $\mathrm{Z}=92$ ). Given that the parameters in the formula were determined from a single fit to a wide range of $Z$ with a wide range of material thicknesses, there is reason to believe that these parameters could differ for scattering specifically in liquid argon with $l \approx X_{0}$. There is also reason to believe that these parameters might be momentum-dependent for particles with $\beta<1$, which is the case for some of the contained muons in this analysis. 
In order to re-tune these parameters for liquid argon, a large sample of muons are simulated with GEANT4 ${ }^{1}$ [14] in the MicroBooNE TPC and their true angular scatters are used in a fit, with $l=X_{0}$. The reason for using $l=X_{0}$ is that the Highland formula simplifies to remove its dependence on $\epsilon$

$$
\sigma_{o}^{\mathrm{HL}}=\frac{S_{2}}{p \beta c} .
$$

The $S_{2}$ parameter in equation 2.3 is fit for as a function of true muon momentum at each scatter, in order to explore the $\beta$ dependence of this parameter. The fitted parameter value as a function of true momentum is shown in figure 3 .

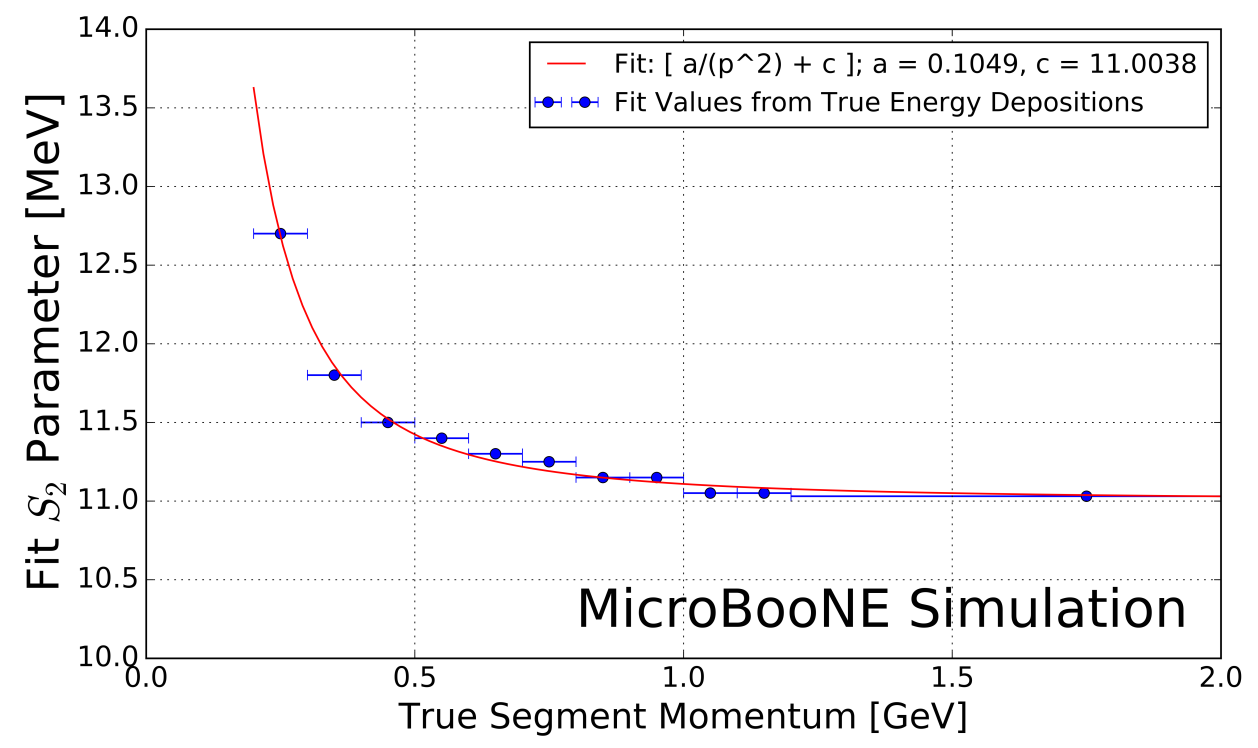

Figure 3. Fitted Highland parameter $S_{2}$ as a function of true segment momentum for $\ell=X_{0}$ simulated muons in the MicroBooNE LArTPC. Blue x- error bars indicate the true momentum bin width with data points drawn at the center of each bin. Shown in red is a fit to these data points with functional form $a \times p^{-2}+c$, with best fit values for parameters $a$ and $c$ shown in the legend.

The fitted value of $S_{2}$ is always less than the nominal $13.6 \mathrm{MeV}$ for momentum greater than $0.25 \mathrm{GeV} / \mathrm{c}$ and asymptotically approaches a constant at higher momentum (where $\beta=1$ ) of about 11.0 MeV. The value increases in the momentum region where $\beta<1$. Shown in red is a fit to these data points with functional form $a \times p^{-2}+c$, with best fit values for floating parameters $a$ and $c$ being $0.105 \mathrm{MeV}^{3} c^{-2}$ and $11.004 \mathrm{MeV}$ respectively. This functional form is chosen because it captures the trend in the fit value of $S_{2}$ with respect to momentum, and asymptotically approaches a constant value when $\beta$ approaches 1 . This function, used as a replacement for the $S_{2}$ parameter in the Highland formula, will henceforth be referred to as $\kappa(p)$ :

$$
\kappa(p)=\frac{0.105}{p^{2}} \mathrm{MeV}^{3} c^{-2}+11.004 \mathrm{MeV} .
$$

${ }^{1}$ The GEANT4 version used in this simulation is 4.9.6. 
To visualize the Highland formula for $\ell=X_{0}$ both before and after the $\kappa(p)$ replacement, see figure 4. It is recommended that future LArTPC experiments use this parameterization of the Highland formula, or at the very least conduct their own studies to tune the Highland formula for scattering in argon. This formulation can also be checked in LAr-based test-beam experiments such as LArIAT [15].

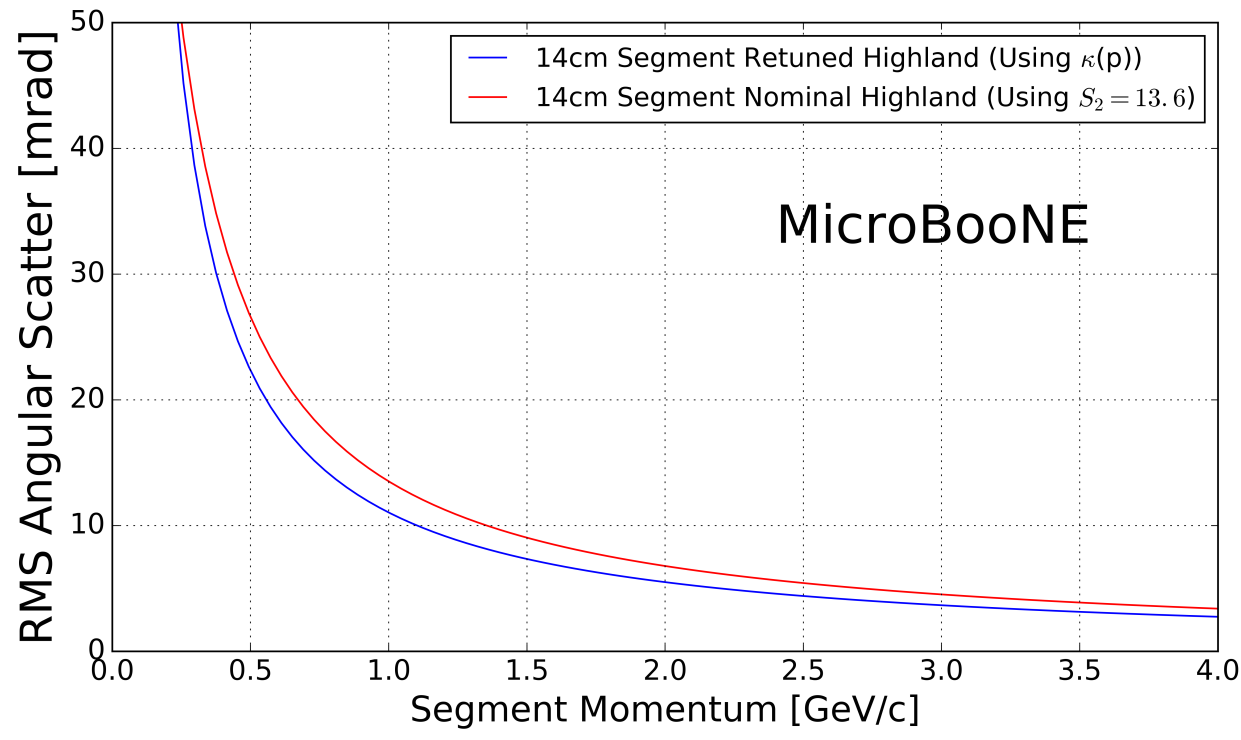

Figure 4. The Highland scattering RMS $\sigma_{o}^{\mathrm{HL}}$ for $14 \mathrm{~cm}$ segment lengths and $\sigma_{o}^{\text {res }}=0$ as a function of true momentum before and after tuning. In red is shown equation 2.3 (the nominal Highland formula using $S_{2}=13.6 \mathrm{MeV}$ ) and in blue is the retuned Highland formula (replacing $S_{2}$ with $\kappa(p)$ ).

With $\ell=X_{0}$, the form of the Highland equation used in this analysis is therefore

$$
\sigma_{o}^{\mathrm{RMS}}=\sqrt{\left(\sigma_{o}\right)^{2}+\left(\sigma_{o}^{\mathrm{res}}\right)^{2}}=\sqrt{\left(\frac{\kappa(p)}{p \beta c}\right)^{2}+\left(\sigma_{o}^{\mathrm{res}}\right)^{2}} .
$$

\section{MCS implementation using the maximum likelihood method}

This section explains in detail how the phenomenon of multiple Coulomb scattering is used to determine the momentum of a muon track reconstructed in a LArTPC. In general, the approach is as follows:

1. The three-dimensional track is divided into segments of configurable length.

2. The scattering angles between consecutive segments are measured.

3. Those angles combined with the modified, tuned Highland formula (equation 2.5) are used to build a likelihood that the particle has a specific momentum, taking into account energy loss in upstream segments of the track. 

computed momentum.

Each of these steps is discussed in detail in the following subsections.

\subsection{Track segmentation and scattering angle computation}

Track segmentation refers to the subdivision of three-dimensional reconstructed trajectory points of a reconstructed track into portions of definite length. In this analysis, the tracks are automatically reconstructed by a projection matching algorithm [16] run on the output of MicroBooNE's Pandorabased neutrino event reconstruction chain [17]. The algorithm constructs the three-dimensional trajectory points by combining two-dimensional hits reconstructed from signals on the different wire planes along with timing information from the photomultiplier tubes. The segmentation process begins at the start of the track, and iterates through the trajectory points in order, defining segment start and stop points based on the straight-line distance between them. There is no overlap between segments. Given the subset of the three-dimensional trajectory points that corresponds to one segment of the track, a three-dimensional linear fit is applied to the data points, weighting all trajectory points equally in the fit. In this analysis, a segment length of $14 \mathrm{~cm}$ is used, which is a tunable parameter that has been chosen as described in the derivation of $\kappa(p)$ (equation 2.4).

With the segments defined, the scattering angles between the linear fits from adjacent segments are computed. A coordinate transformation is performed such that the $z^{\prime}$ direction is oriented along the direction of the linear fit to the first of the segment pair. The $x^{\prime}$ and $y^{\prime}$ coordinates are chosen such that all of $x^{\prime}, y^{\prime}$, and $z^{\prime}$ are mutually orthogonal and right-handed, as shown in figure 2 . The scattering angles with respect to the $x^{\prime}$ direction and the $y^{\prime}$ direction are computed as input to the MCS algorithm. Only the scattering angle with respect to the $x^{\prime}$ direction is drawn in figure 2.

\subsection{Maximum likelihood theory}

The normal probability distribution for a scattering angle in either the $x^{\prime}$ or $y^{\prime}$ direction, $\Delta \theta$, with an expected Gaussian uncertainty $\sigma_{o}$ and mean of zero is given by

$$
f_{X}(\Delta \theta)=\left(2 \pi \sigma_{o}^{2}\right)^{-\frac{1}{2}} \exp \left[-\frac{1}{2}\left(\frac{\Delta \theta}{\sigma_{o}}\right)^{2}\right] .
$$

Here, $\sigma_{o}$ is the RMS angular deflection computed by the modified, tuned Highland formula (equation 2.5), which is a function of the momentum and the length of that segment. Since energy is lost between segments along the track, $\sigma_{o}$ increases for each angular measurement along the track. We therefore replace $\sigma_{o}$ with $\sigma_{o, j}$, where $j$ is an index representative of the segment.

To obtain the likelihood, we take the product of $f_{X}\left(\Delta \theta_{j}\right)$ over all $n$ of the $\Delta \theta_{j}$ segment-tosegment scatters along the track. This product can be written as

$$
L\left(\sigma_{o, 1}, \ldots, \sigma_{o, n} ; \Delta \theta_{1}, \ldots, \Delta \theta_{n}\right)=(2 \pi)^{-\frac{n}{2}} \times \prod_{j=1}^{n}\left(\sigma_{o, j}\right)^{-1} \times \exp \left[-\frac{1}{2} \sum_{j=1}^{n}\left(\frac{\Delta \theta_{j}}{\sigma_{o, j}}\right)^{2}\right] .
$$


imize the negative $\log$ likelihood. Inverting the sign and taking $\ln (L)$ gives an expression that is related to a $\chi^{2}$ variable:

$$
-l\left(\sigma_{o, 1}, \ldots, \sigma_{o, n} ; \Delta \theta_{1}, \ldots, \Delta \theta_{n}\right)=-\ln (L)=\frac{n}{2} \ln (2 \pi)+\sum_{j=1}^{n} \ln \left(\sigma_{o, j}\right)+\frac{1}{2} \sum_{j=1}^{n}\left(\frac{\Delta \theta_{j}}{\sigma_{o, j}}\right)^{2} .
$$

\subsection{Maximum likelihood implementation}

Given a set of angular deflections in the $x^{\prime}$ and $y^{\prime}$ directions for each segment as described in section 3.1 a scan is done over the postulated initial energy, $E_{t}$, in steps of $1 \mathrm{MeV}$ up to $7.5 \mathrm{GeV}$. The step with the smallest negative log likelihood (equation 3.3) is chosen as the MCS energy. Equation 3.3 includes a $\sigma_{o, j}$ term that changes for consecutive segments because their associated energy is decreasing. The energy of the $j$ th segment is related to $E_{t}$ by

$$
E_{j}=E_{t}-\Delta E_{j},
$$

where $\Delta E_{j}$ is the energy loss upstream of this segment, computed by integrating the muon stopping power curve given by the Bethe-Bloch equation described by the Particle Data Group (PDG) [18] along the length of track upstream of this segment. Equation 3.4 introduces a minimum allowable track energy determined by the range of the track, as $E_{j}$ must remain positive. The use of the Bethe-Bloch equation to determine $\Delta E_{j}$ impacts the MCS algorithm resolution for fully contained tracks, but does not for exiting tracks where much of the ionization energy loss is not visible. This value of segment energy, $E_{j}$, is converted to a momentum $p$ with the relativistic energy-momentum relation assuming the muon mass, and is then used to predict the RMS angular scatter for that segment $\left(\sigma_{o}\right)$ by way of equation 2.5 .

\section{Range-based energy validation from simulation}

In order to quantify the performance of the MCS energy estimation method on fully contained muons in data, an independent determination of energy is needed. Range-based energy, $E_{\text {range }}$ is used here because the true energy $E_{\text {true }}$ will not be known in analyzing detector data. The stopping power of muons in liquid argon is well described by the continuous slowing-down approximation (CSDA) by the Particle Data Group, and agrees with data at the sub-percent level [19-21]. By using a linear interpolation between points in the stopping power table of ref. [20], the length of a track can be used to reconstruct the muon's total energy with good accuracy. A simulated sample of fully contained BNB neutrino-induced muons longer than one meter is used to quantify the bias and resolution for the range-based energy estimation technique. The range is defined as the straightline distance between the true starting point and true stopping point of a muon, even though the trajectories are not perfectly straight lines. The bias and resolution are computed in bins of true total energy of the muons by fitting a Gaussian function to a distribution of the fractional energy difference $\left(E_{\text {Range }}-E_{\text {True }}\right) /\left(E_{\text {True }}\right)$ in each bin. The mean of each Gaussian yields the bias for that true energy bin, and the width indicates the resolution. Figure 5 shows the bias and resolution for the range-based energy reconstruction method. The bias is less than $1 \%$ and the resolution for this method of energy reconstruction increases slightly with true muon energy but remains on 

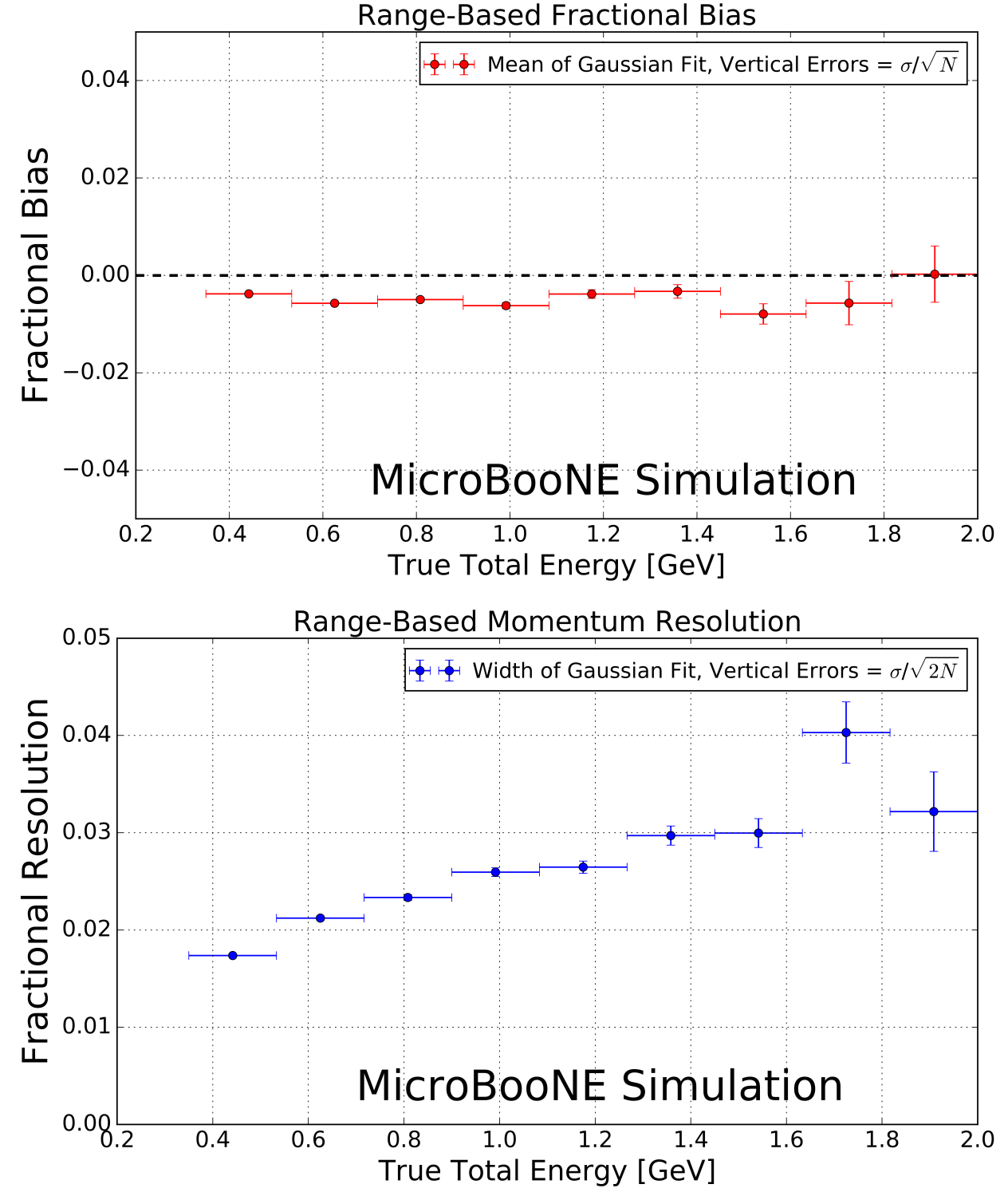

Figure 5. Range-based energy fractional bias (top) and resolution (bottom) from a sample of simulated fully contained BNB neutrino-induced muons using true starting and stopping positions of the track. The bias is less than $1 \%$ and the resolution is below $\approx 4 \%$.

the order of (2-4)\%. This result demonstrates that range-based energy (and therefore range-based momentum) is a good estimator of the true energy (momentum) of a reconstructed contained muon track in data, assuming that the track is well reconstructed in terms of length. 


\subsection{Input sample}

This part of the analysis is based on triggered neutrino interaction events in MicroBooNE data corresponding to $\approx 5 \times 10^{19}$ protons on target, which is a small subset $(<10 \%)$ of the nominal protons on target scheduled to be delivered to the detector. These events are run through a fully automated reconstruction chain that produces reconstructed objects including three-dimensional neutrino interaction points (vertices), three-dimensional tracks (as described in section 3.1) for each outgoing secondary particle from the interaction, and PMT-reconstructed optical flashes from the interaction scintillation light. The fiducial volume used in this analysis is defined in section 1.

\subsection{Event selection}

The following selection criteria are placed on the reconstructed objects to select $v_{\mu}$ charged-current interactions in which a candidate muon track exiting the interaction vertex is fully contained within the fiducial volume:

1. The event must have at least one bright optical flash, reconstructed from PMT timing signals, in coincidence with the expected BNB-neutrino arrival time.

2. Two or more reconstructed tracks must originate from the same reconstructed vertex within the fiducial volume.

3. The $z$ coordinate of the optical flash, as determined by the pulse height and timing of signals in the 32 PMTs, must be within $70 \mathrm{~cm}$ of any point on the $z$ projection of the candidate muon track.

4. For events with exactly two tracks originating from the vertex, additional calorimetric criteria are applied to mitigate backgrounds from cosmic muons that arrive in time with the passage of the beam, then stop and decay to an electron that is reconstructed as a track.

5. The longest track originating from the vertex is assumed to be a muon, and it must be fully contained within the fiducial volume.

6. The length of the longest track must be $>1 \mathrm{~m}$ in order to have sufficient sampling points in the MCS likelihood to obtain a reasonable estimate of momentum.

These selection criteria are chosen to select a sample of tracks with high purity. In this sample of MicroBooNE data, 598 events (tracks) remain after all selections. The low statistics in this sample is due to the size of the input sample and the low efficiency associated with the applied high-purity selection, described in section 5.1. Each of these events (tracks) was scanned by hand with a $2 \mathrm{D}$ interactive event display showing the raw wire signals of the interaction from each wire plane, with the 2D projection of the reconstructed muon track and vertex overlaid. The scanning was done to ensure the track is well reconstructed with start point close to the reconstructed vertex and end point close to the end of the visible wire-signal track in all three planes. During the scanning, obvious mis-identification topologies were removed. An example of such a topology is a stopping cosmic-ray muon decaying into an electron. After rejecting events (tracks) based on hand scanning, 396 tracks remain for analysis. 
The Highland formula indicates that distributions of angular deviations of the track, segment by segment, in both the $x^{\prime}$ and $y^{\prime}$ directions divided by the width predicted from the Highland equation $\sigma_{o}^{\text {RMS }}$ (equation 2.5) should be Gaussian with a width of unity. In order to calculate the momentum $p$ in the Highland equation, $p$ for each segment is computed with equation 3.4, where $E_{t}$ comes from the converged MCS-computed momentum of the track. For each consecutive pair of segments in this sample of 396 tracks, the angular scatter divided by the Highland expected RMS (including detector resolution term, $\sigma_{o}^{\text {res }}$ ) is an entry in the area-normalized distribution shown in figure 6 . These 396 tracks have on average 12 segments each, therefore this histogram has approximately $396 \times 12 \times 2=9504$ entries. The additional factor of 2 comes from angular scatters both in the $x^{\prime}$ and $y^{\prime}$ directions. The distribution has an RMS of unity, thus validating the MCS technique used in this analysis.

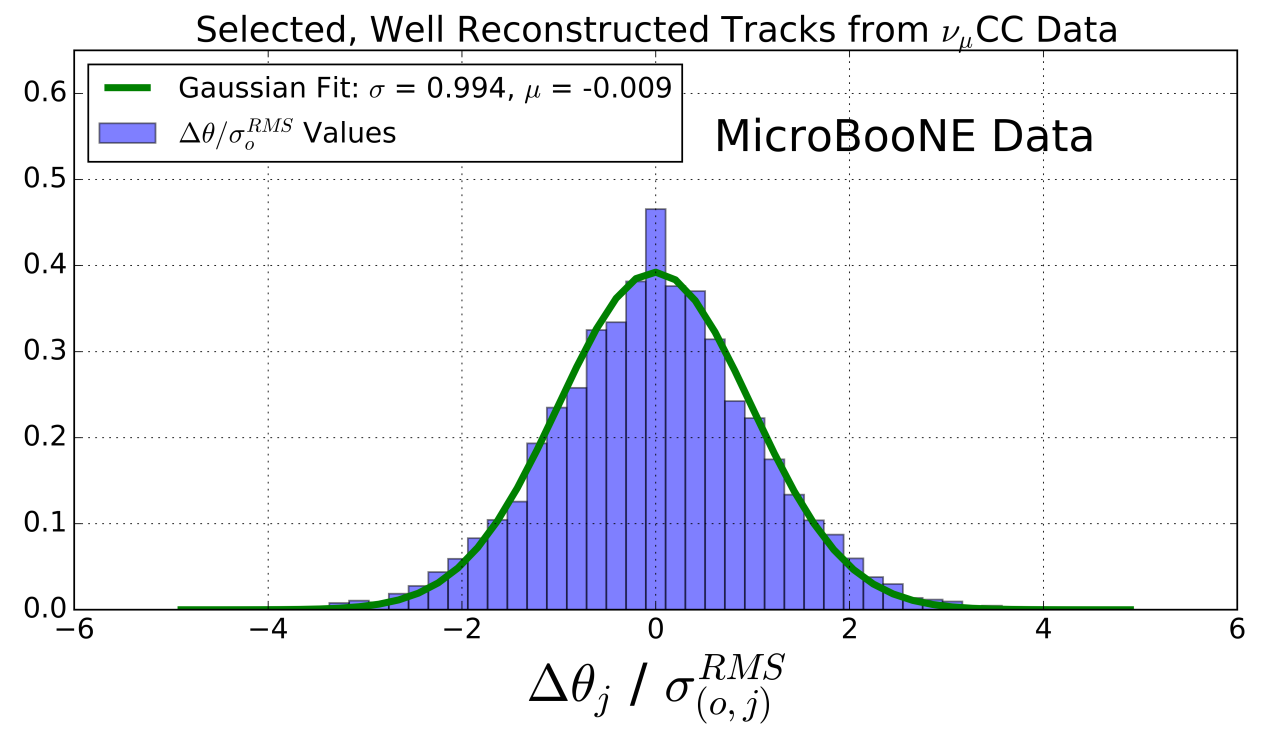

Figure 6. Segment-to-segment measured angular scatters in both the $x^{\prime}$ and $y^{\prime}$ directions divided by the width $\sigma_{o}^{\mathrm{RMS}}$ predicted by the Highland formula (equation 2.1) for the automatically selected beam neutrinoinduced fully contained muon sample in MicroBooNE data after hand scanning to remove poorly reconstructed tracks and obvious mis-identification topologies.

\subsection{MCS momentum validation}

MCS momentum versus range-based momentum for this sample of 396 tracks is shown in figure 7. The fractional bias and resolution as a function of range-based momentum for this sample is shown in figure 8. In order to compute this bias and resolution, distributions of fractional inverse momentum difference $\left(p_{\text {MCS }}^{-1}-p_{\text {Range }}^{-1}\right) /\left(p_{\text {Range }}^{-1}\right)$ in bins of range-based momentum $p_{\text {Range }}$ are fit to Gaussian functions, where the mean of the fit determines the bias while the width of the fit determines the resolution for that bin. Inverse momentum is used here because the binned distributions are more Gaussian since the Highland formula measures inverse momentum in terms of 

distributions yields similar results. Also shown in this figure are the bias and resolutions for a simulated sample consisting of a full BNB simulation with CORSIKA-generated [22] cosmic overlays passed through an identical reconstruction and event selection chain. Rather than hand scanning this sample, true simulation information is used by requiring the longest reconstructed track to be matched well to the true starting and stopping point of the muon from the $v_{\mu} \mathrm{CC}$ interaction. This removes any mis-identifications or interference from the simulated cosmics.

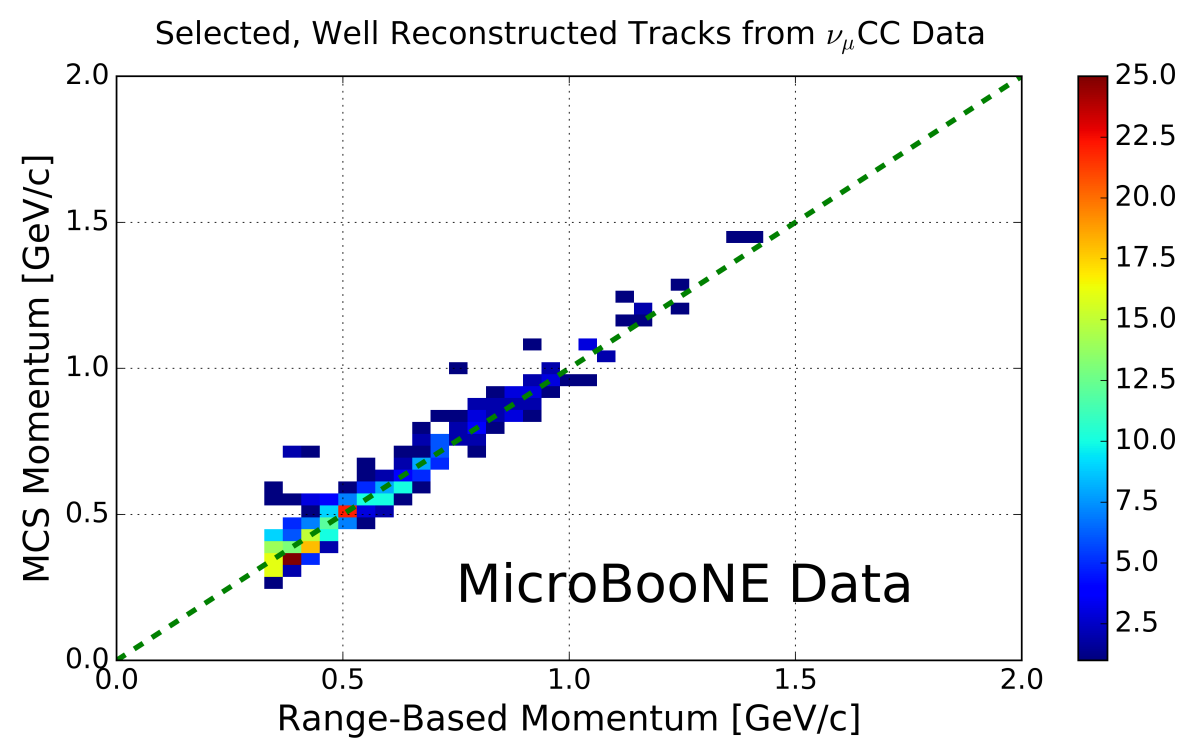

Figure 7. MCS-computed momentum versus range momentum for the automatically selected beam neutrino-induced fully contained muon sample in MicroBooNE data after hand scanning to remove poorly reconstructed tracks and obvious mis-identification topologies. The color $(\mathrm{z})$ scale indicates number of tracks.

Figure 8 indicates a bias in the MCS momentum calculation on the order of a few percent, with a resolution that improves from about $10 \%$ for contained reconstructed tracks in data and simulation with range momentum around $0.45 \mathrm{GeV} / \mathrm{c}$ (which corresponds to a length of about $1.5 \mathrm{~m}$ ) to below $5 \%$ for contained reconstructed tracks in data and simulation with range momentum about $1.15 \mathrm{GeV} / \mathrm{c}$ (which corresponds to a length of about 4.6 meters). Resolution improving with length of track is expected; the longer the track, the more angular scattering measurements can be made to improve the likelihood. The bias and resolutions show reasonable agreement between data and simulation.

\subsection{Impact of Highland formula tuning}

In order to examine the impact of the Highland formula tuning described in section 2.1, the fractional bias and resolution on the simulated sample of contained muons described in section 5.4 both with the nominal Highland formula (equation 2.2) and with the retuned Highland formula (equation 

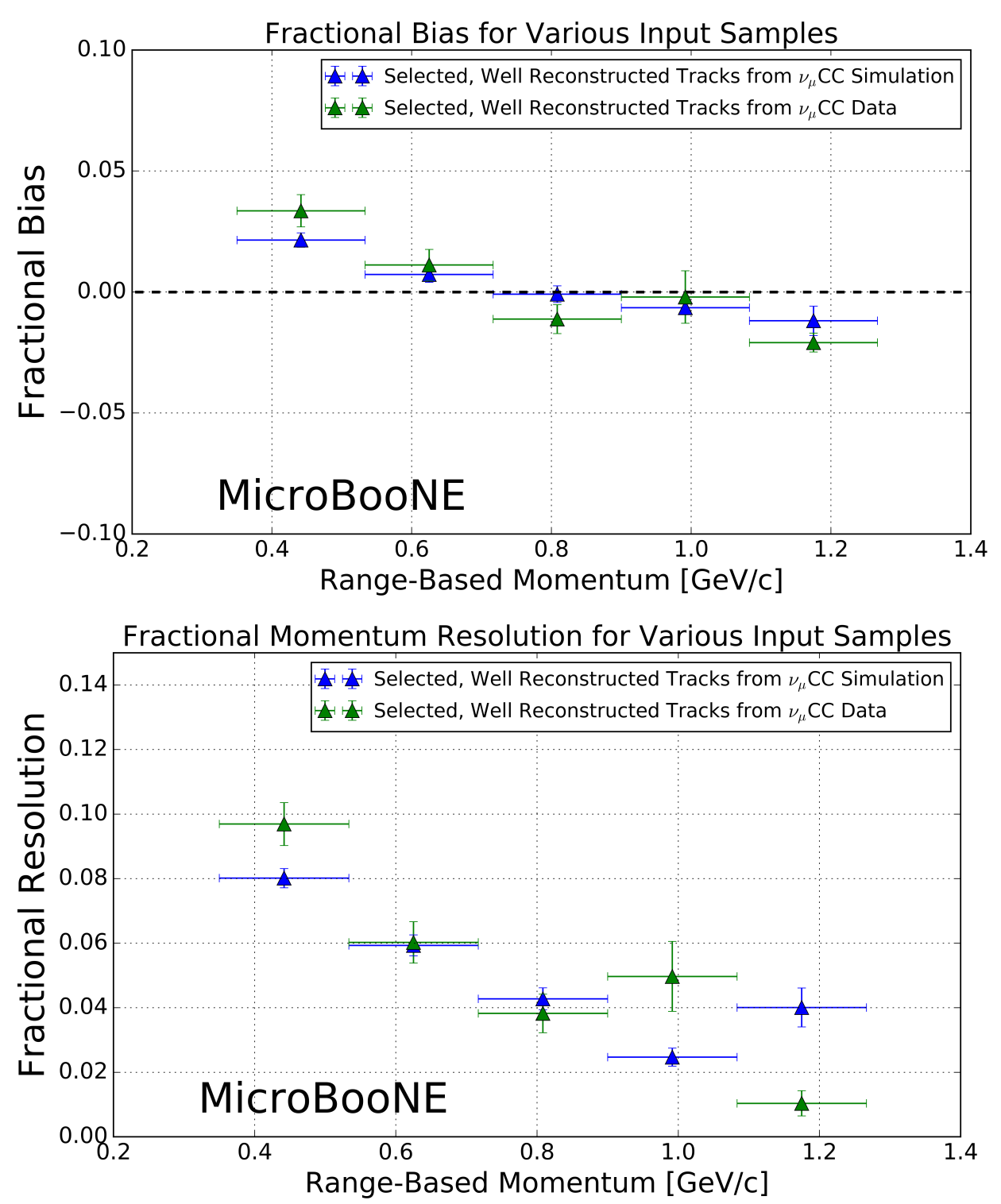

Figure 8. Inverse momentum difference (as defined in the text) fractional bias (top) and resolution (bottom) for automatically selected contained $v_{\mu} \mathrm{CC}$-induced muons from full simulated BNB events with cosmic overlay where the track matches with the true muon track (blue), and automatically selected and handscanned (see text) contained $v_{\mu} \mathrm{CC}$-induced muons from MicroBooNE data (green). 

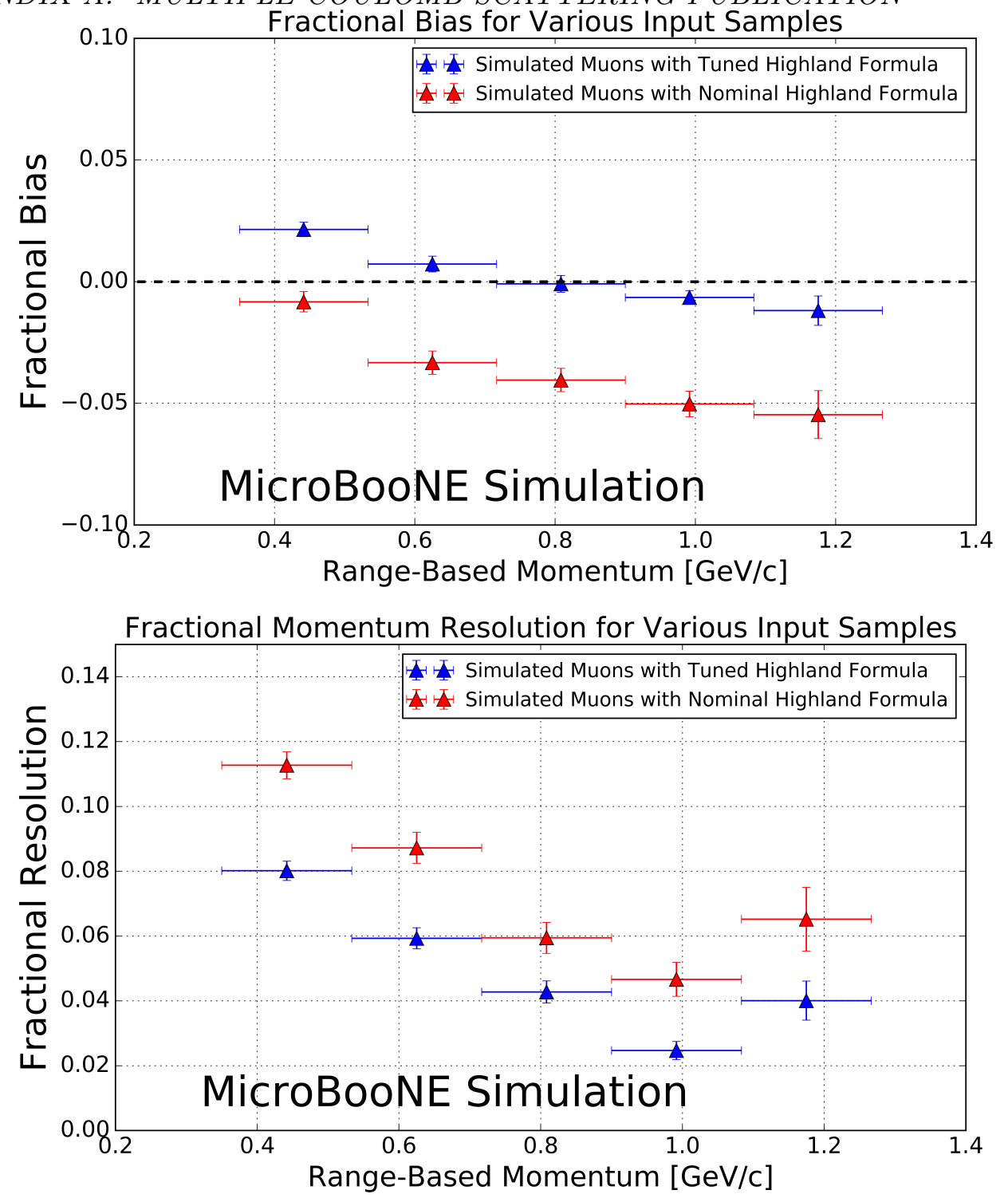

Figure 9. Inverse momentum difference (as defined in the text) fractional bias (top) and resolution (bottom) for automatically selected contained $v_{\mu}$ CC-induced muons from full simulated BNB events with cosmic overlay where the track matches with the true muon track both using the nominal Highland formula (equation 2.2) (red) and the retuned Highland formula (equation 2.5) (blue).

2.5) are shown in figure 9. Tuning the Highland formula improves the magnitude of the fractional bias to below $2 \%$, and improves the fractional resolution by (2-3)\%, with the most improvement at the lowest momenta. 
In this section we quantify the MCS algorithm performance on a sample of well reconstructed exiting muon tracks in simulated BNB $v_{\mu} \mathrm{CC}$ interactions within the MicroBooNE detector. The tracks are automatically reconstructed by the same Pandora algorithm as described in section 3.1, and all tracks have a length of at least $1 \mathrm{~m}$ within the TPC. This simulation does not include space-charge effects. Approximately half of muons from $v_{\mu} \mathrm{CC}$ interactions within the specified fiducial volume exit the TPC, and about two thirds of those muons have at least one meter of track contained inside of the TPC. The relationship between the MCS and the true momenta at the beginning of the track as given by simulation for this sample of 28,000 exiting muon tracks is shown in figure 10.

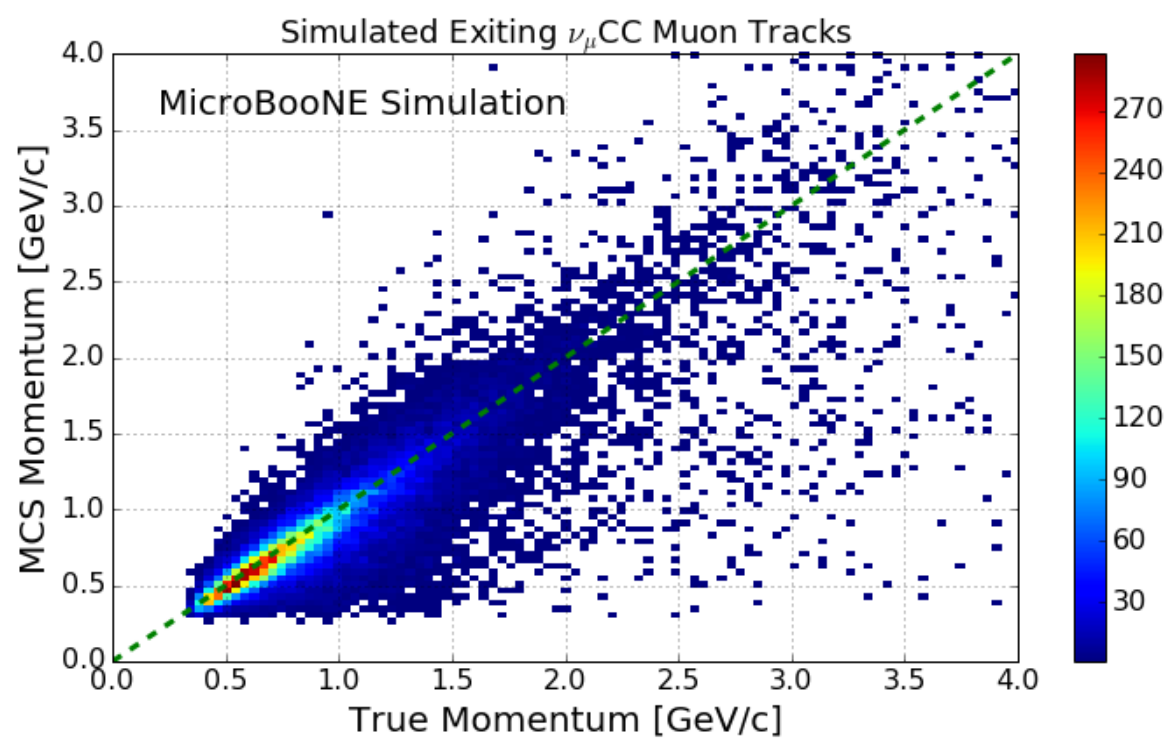

Figure 10. MCS-computed momentum versus true momentum for the sample of simulated exiting muons from BNB $v_{\mu}$ CC interactions in MicroBooNE with at least one meter of track contained within the TPC. The color $(\mathrm{z})$ scale indicates number of tracks.

The distribution of $\left(p_{\mathrm{MCS}}^{-1}-p_{\text {true }}^{-1}\right) /\left(p_{\text {true }}^{-1}\right)$ is shown for four representative bins of true momentum in figure 11, along with the Gaussian fit to each distribution. Low-momentum tails where the MCS momentum is underestimated due to poor track reconstruction lie outside the fitted Gaussian function.

The fractional bias and resolution as a function of true momentum are shown in figure 12 . The bias is below $4 \%$ for all momenta, and the resolution is $\approx 14 \%$ in the relevant momentum region for muon from the $\mathrm{BNB} v_{\mu} \mathrm{CC}$ interactions (below $2 \mathrm{GeV} / \mathrm{c}$ muon momentum). The resolution worsens for muon momenta above this region because the angular scatters begin to be comparable with the detector resolution term of $3 \mathrm{mrad}$. The resolution improves for longer lengths of track contained, with $10 \%$ resolution for muons with $p<2 \mathrm{GeV} / \mathrm{c}$ with more than 3.5 meters contained. 


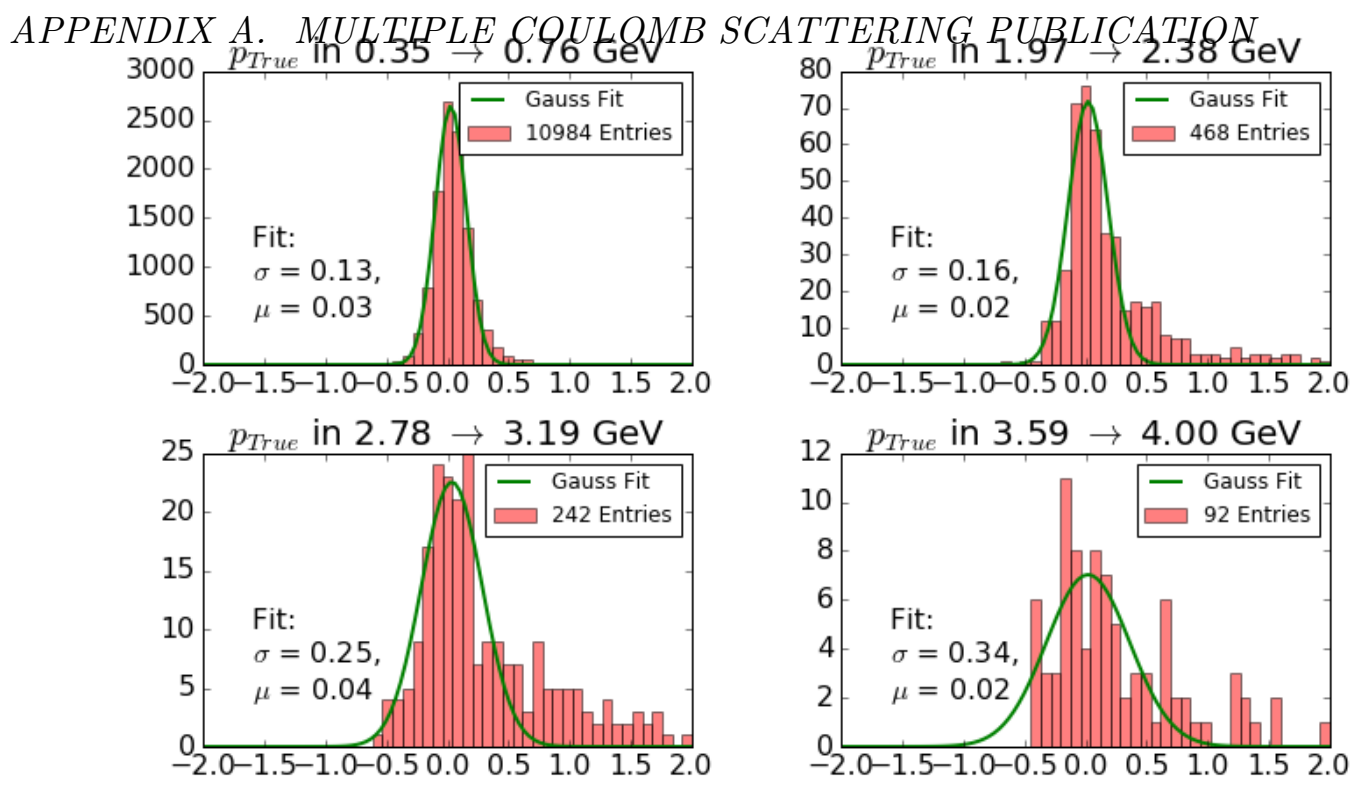

Figure 11. Fractional momentum difference for a few representative bins of true momentum for a sample of simulated exiting muon tracks. The y-axis is number of tracks, and the $\mathrm{x}$-axis is $\left(p_{\mathrm{MCS}}^{-1}-p_{\text {true }}^{-1}\right) /\left(p_{\text {true }}^{-1}\right)$.

The mean length of track contained for muons in this analysis is $212 \mathrm{~cm}$.

\section{Conclusions}

We have described a multiple Coulomb scattering maximum likelihood method for estimating the momentum of a three dimensional reconstructed track in a LArTPC and have provided motivation for development of such a technique. Using simulation, we have shown that the standard Highland formula should be re-tuned specifically for scattering in liquid argon. After validating range-based momentum-determination techniques with MicroBooNE simulation, we have demonstrated the accuracy and precision of the MCS-based momentum reconstruction in MicroBooNE data by comparing its performance to the range-based method. For 398 fully-contained BNB $v_{\mu} \mathrm{CC}$-induced muons, the MCS method exhibits a fractional bias below $3 \%$ and a momentum resolution below $10 \%$, agreeing with simulation predictions. Using simulation of a separate sample of uncontained muon tracks in MicroBooNE with at least one meter contained in the active volume, the MCS-based reconstruction is shown to produce a fractional bias less than $4 \%$ and a momentum resolution of better than $15 \%$ for muons in the relevant BNB energy region of below $2 \mathrm{GeV}$.

\section{Acknowledgments}

This material is based upon work supported by the following: the U.S. Department of Energy, Office of Science, Offices of High Energy Physics and Nuclear Physics; the U.S. National Science Foundation; the Swiss National Science Foundation; the Science and Technology Facilities Council 

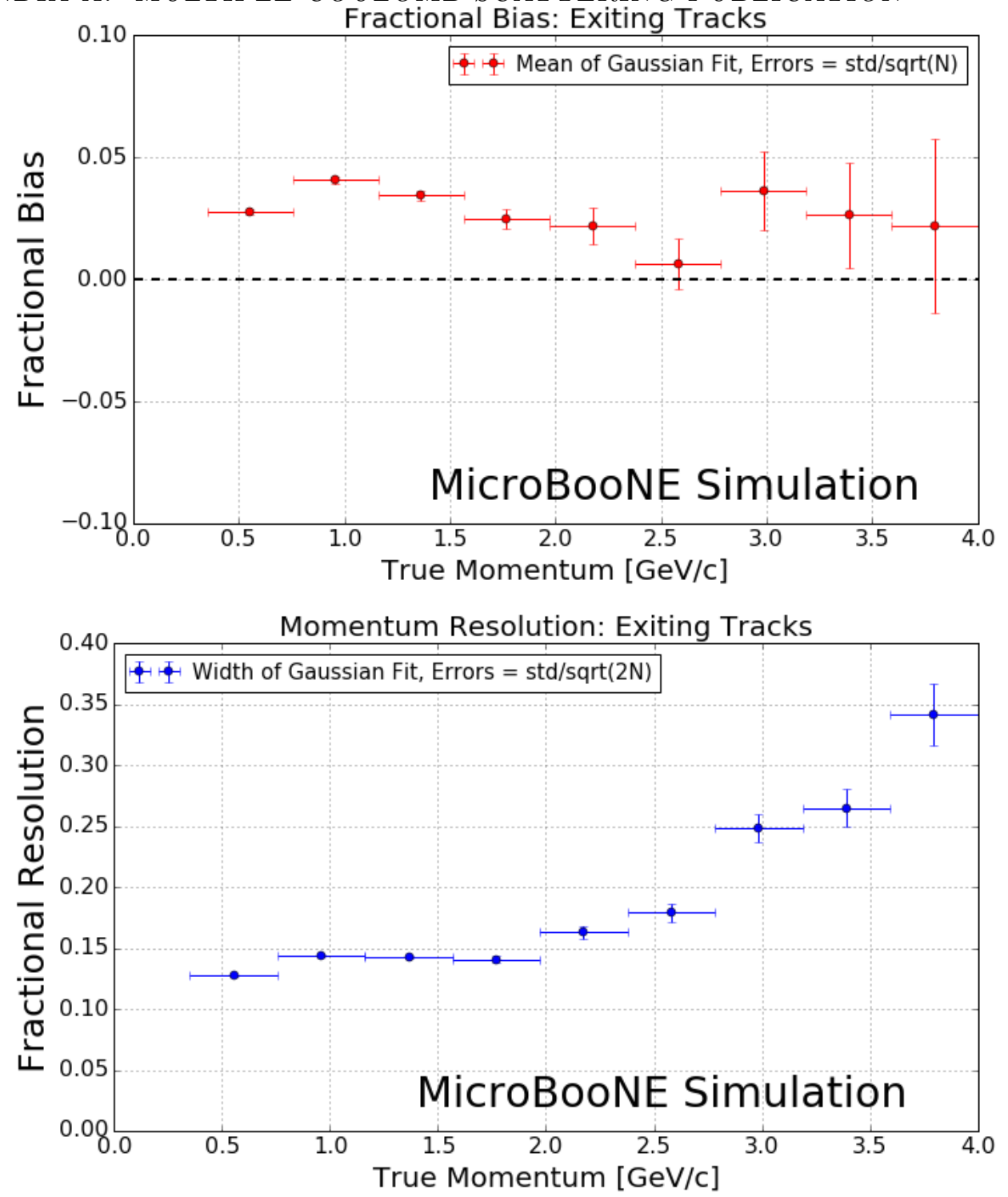

Figure 12. MCS momentum fractional bias (top) and resolution (bottom) as a function of true momentum from a sample of exiting reconstructed muon tracks.

of the United Kingdom; and The Royal Society (United Kingdom). Additional support for the laser calibration system and cosmic ray tagger was provided by the Albert Einstein Center for Fundamental Physics. Fermilab is operated by Fermi Research Alliance, LLC under Contract No. DE-AC02-07CH11359 with the United States Department of Energy. 
[1] A. A. Aguilar-Arevalo et al. [MiniBooNE Collaboration], Improved Search for $\bar{v}_{\mu} \rightarrow \bar{v}_{e}$ Oscillations in the MiniBooNE Experiment, Phys. Rev. Lett. 110, 161801 (2013). [arXiv:1207.4809 [hep-ex], arXiv:1303.2588 [hep-ex]].

[2] C. Adams et al. [LArTPC Collaboration], LAr1-ND: Testing Neutrino Anomalies with Multiple LArTPC Detectors at Fermilab, arXiv:1309.7987 [physics.ins-det].

[3] F. Arneodo et al. [ICARUS Collaboration], The ICARUS experiment: A Second generation proton decay experiment and neutrino observatory at the Gran Sasso Laboratory, hep-ex/0103008.

[4] R. Acciarri et al. [DUNE Collaboration], Long-Baseline Neutrino Facility (LBNF) and Deep Underground Neutrino Experiment (DUNE) : Volume 1: The LBNF and DUNE Projects, arXiv:1601.05471 [physics.ins-det].

[5] R. Acciarri et al. [MicroBooNE Collaboration], Design and Construction of the MicroBooNE Detector, Journal of Instrumentation 12, P02017 (2017). arXiv:1612.05824 [physics.ins-det].

[6] A. A. Aguilar-Arevalo et al. [MiniBooNE Collaboration], The Neutrino Flux prediction at MiniBooNE, Phys. Rev. D 79, 072002 (2009) [arXiv:0806.1449 [hep-ex]].

[7] V. L. Highland, Some Practical Remarks on Multiple Scattering, Nucl. Instrum. Methods 129 (1975) 104-120.

[8] G. R. Lynch and O. I. Dahl, Nucl. Instrum. Methods Section B (Beam Interactions with Materials and Atoms) B58, 6 (1991).

[9] K. Kodama et al. [DONUT Collaboration], Observation of tau neutrino interactions, Phys. Lett. B 504, 218 (2001) [hep-ex/0012035].

[10] N. Agafonova et al. [OPERA Collaboration], Momentum measurement by the Multiple Coulomb Scattering method in the OPERA lead emulsion target, New J. Phys. 14, 013026 (2012) [arXiv:1106.6211 [physics.ins-det]].

[11] G. Giacomelli [MACRO Collaboration], Neutrino physics and astrophysics with the MACRO experiment at the Gran Sasso lab, Braz. J. Phys. 33, 211 (2003) [hep-ex/0210006].

[12] A. Ankowski et al. [ICARUS Collaboration], Measurement of through-going particle momentum by means of multiple scattering with the ICARUS T600 TPC, Eur. Phys. J. C 48, 667 (2006) [hep-ex/0606006].

[13] M. Antonello et al., Muon momentum measurement in ICARUS-T600 LAr-TPC via multiple scattering in few-GeV range, arXiv:1612.07715 [physics.ins-det].

[14] S. Agostinelliet al. Nucl. Instrum. Methods Phys. Res. A506250-303 (2003)

[15] F. Cavanna et al. [LArIAT Collaboration], LArIAT: Liquid Argon In A Testbeam, arXiv:1406.5560 [physics.ins-det].

[16] M. Antonello et al., Precise 3D track reconstruction algorithm for the ICARUS T600 liquid argon time projection chamber detector, Adv. High Energy Phys. 2013, 260820 (2013) [arXiv:1210.5089 [physics.ins-det]].

[17] J. S. Marshall and M. A. Thomson, The Pandora Software Development Kit for Pattern Recognition, Eur. Phys. J. C 75, no. 9, 439 (2015) 

[arXiv:1506.05348 [physics.data-an]].

[18] H. Bichsel, D. E. Groom, S.R. Klein, Passage of Particles Through Matter PDG Chapter 27, Figure 27.1 http://pdg.lbl.gov/2005/reviews/passagerpp.pdf

[19] D. E. Groom, N. V. Mokhov and S. Striganov, Muon Stopping Power and Range Tables: $10 \mathrm{MeV}$ $100 \mathrm{TeV}$ Table 5, http://pdg.lbl.gov/2012/AtomicNuclearProperties/adndt.pdf

[20] Table 289: Muons in Liquid argon (Ar) http://pdg.lbl.gov/2012/AtomicNuclearProperties/ MUON_ELOSS_TABLES/muonloss_289.pdf

[21] Stopping Powers and Ranges for Protons and Alpha Particles, ICRU Report No. 49 (1993); Tables and graphs of these data are available at http://physics.nist.gov/PhysRefData/

[22] D. Heck, J. Knapp, J. N. Capdevielle, G. Schatz, T. Throw, CORSIKA: A Monte Carlo Code to Simulate Extensive Air Showers, Forschungszentrum Karlsruhe Report FZKA 6019 (1998) 